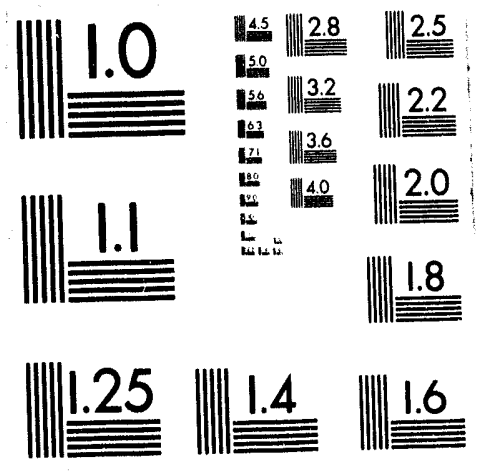



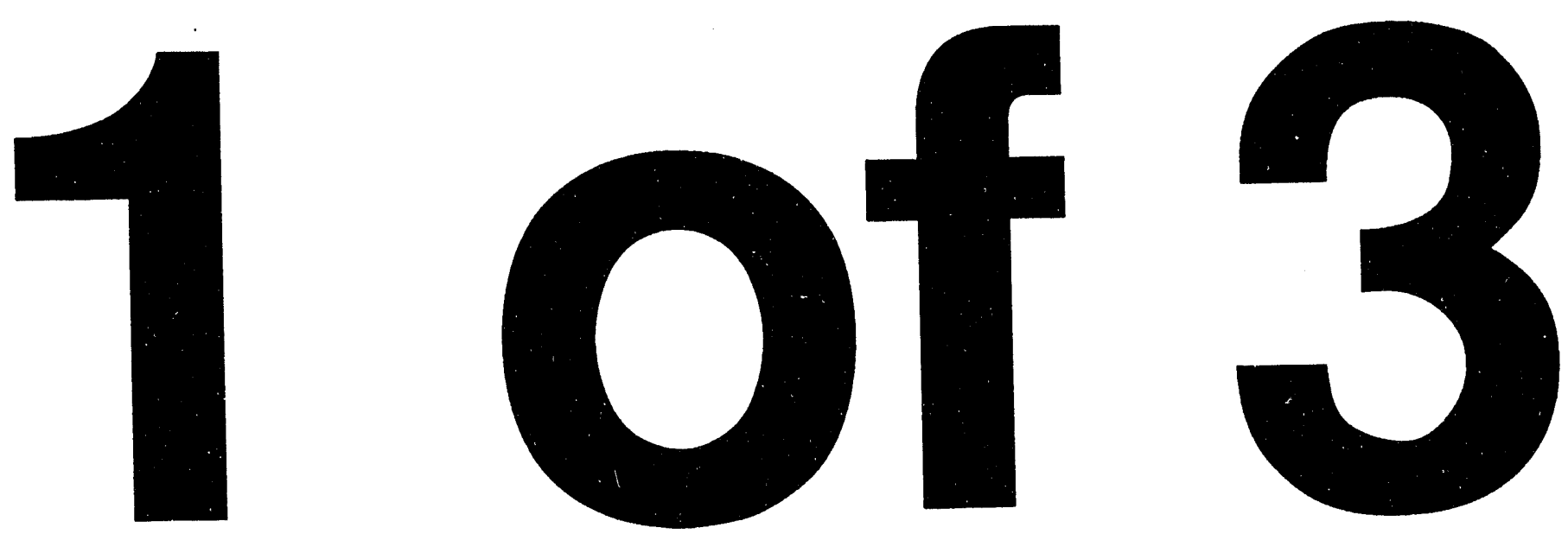


\title{
CPT
}

CHEMICAL PROCESS TECHNOLOGY

WSRC-TR-93-262

Key Words: Data

Risk

Retentlon: Lifetlme

\section{SAVANNAH RIVER SITE \\ GENERIC DATA BASE DEVELOPMENT (U)}

\author{
by \\ C.H. Blanton \\ S.A. Eide
}

Issued: June 30, 1993

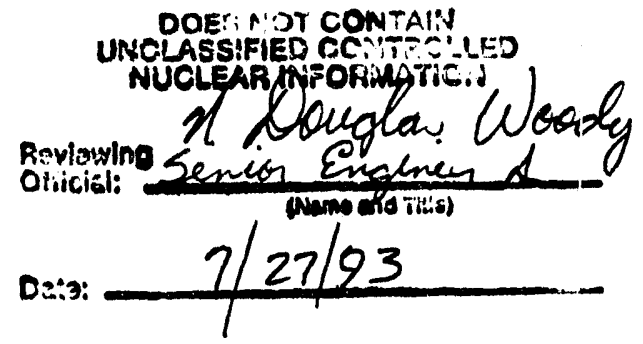

Westinghouse Savannah River Company

Savannah River Site

Aiken, SC 29808

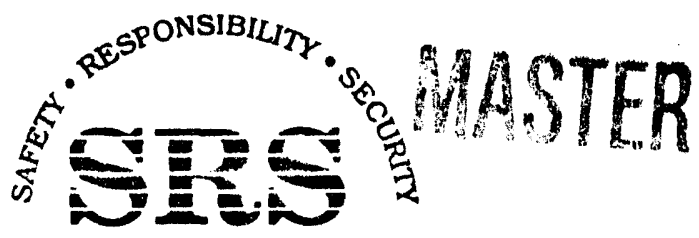

SAVANNAH RIVER SITE

PREPARED FOR THE U.S. DEPARTMENT OF ENERGY UNDER CONTRACT NO. DE-AC09-89SR18035

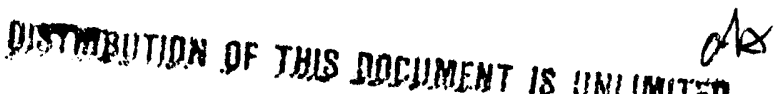


This Page Intentionally Left Blank 
WSRC-TR-93.262

PROJECT: DWPF SER REVISION

DOCUMENT: WSRC-TR-93-262

TITLE: SAVANNAH RIVER SITE GENERIC DATA BASE DEVELOPMENT (U)

APPROVALS:

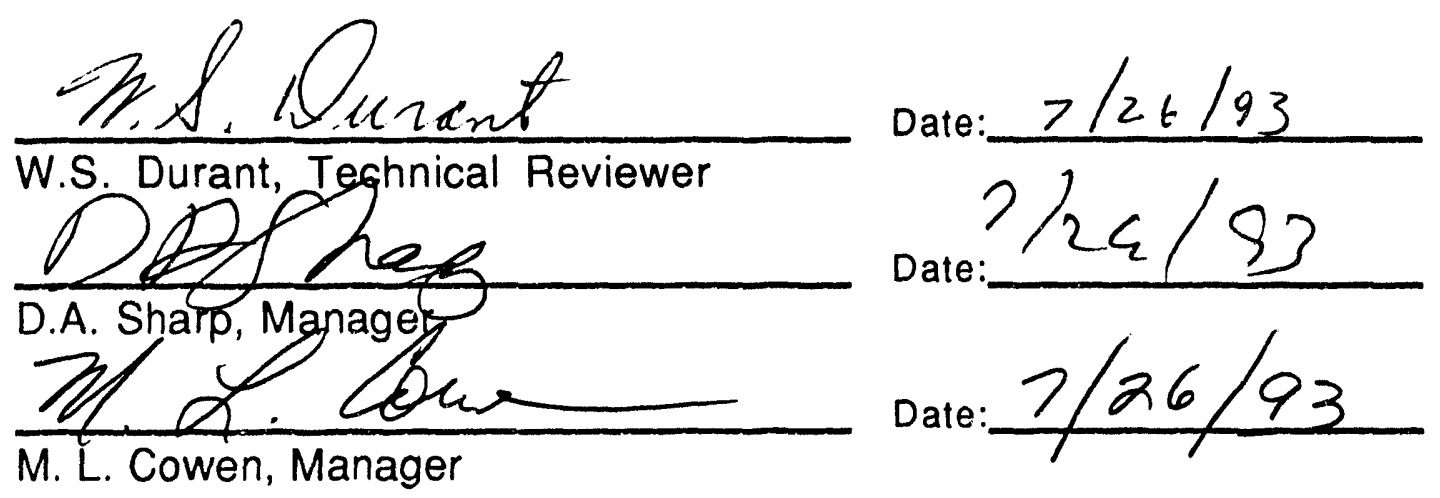

$\mathbf{i} \mathbf{i}$ 


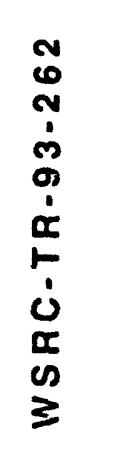

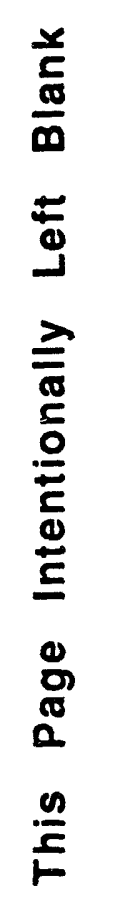




\begin{abstract}
This report describes the results of a project to improve the generic component failure data base for the Savannah River Site (SRS). A representative list of components and failure modes for SRS risk models was generated by reviewing existing safety analyses and component failure data bases and from suggestions from SRS safety analysts. Then sources of data or failure rate estimates were identified and reviewed for applicability. A major source of information was the Nuclear Computerized Library for Assessing Reactor Reliability, or NUCLARR. ${ }^{1}$ This source includes an extensive collection of failure data and failure rate estimates for commercial nuclear power plants. A recent Idaho National Engineering Laboratory report on failure data from the Idaho Chemical Processing Plant was also reviewed. ${ }^{2}$ From these and other recent sources, failure data and failure rate estimates were collected for the components and failure modes of interest. This information was aggregated to obtain a recommended generic failure rate distribution (mean and error factor) for each component failure mode. Results are presented in Table 1 in this report.
\end{abstract}

A major difference between this generic data base and previous efforts is that this effort attempted to base failure rate estimates on actual data (failure events) rather than on existing failure rate estimates. This effort was successful in that over $75 \%$ of the results are now based on actual data. 
WSRC-TR-93-262

This Page Intentionally Left Blank 


\section{TABLE OF CONTENTS}

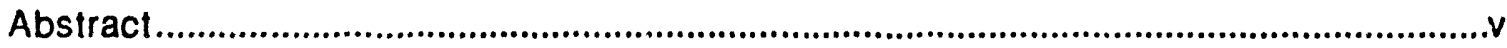

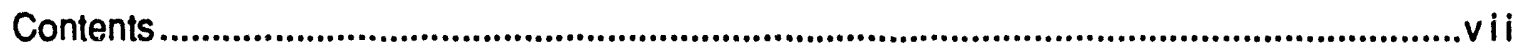

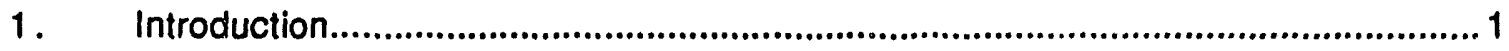

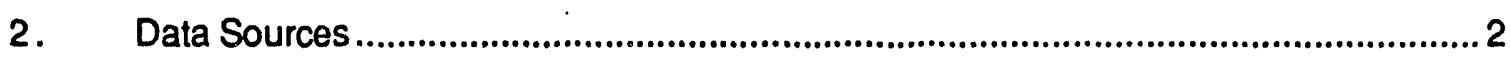

3. Aggregation Routines ......................................................................................... 4

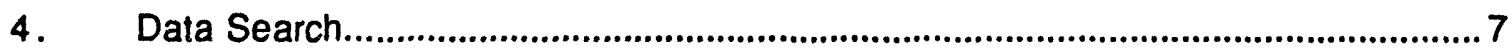

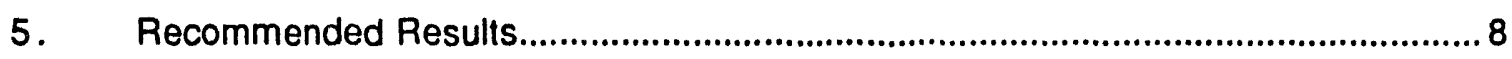

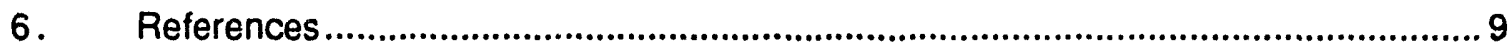

\section{LIST OF TABLES}

1a. Water system recommendod genorlc fallure rates ..........................................12

1b. Chemical process system recommended generic failure rates............................16

1c. Compressed gas system recommended generic failure rates..............................20

1d. HVAC/exhaust system recornmended generic failure rates ................................24

1e. Electrica! distribution system recommended generic failure rates ..................27

1f. Instrumentation and control system recommended generic failure rates............30

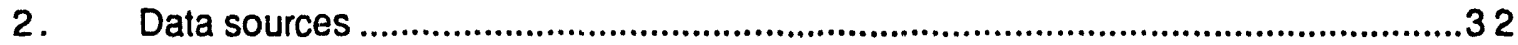

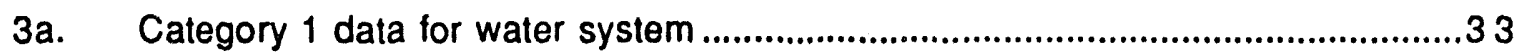

3b. Category 1 data for chemical process sys

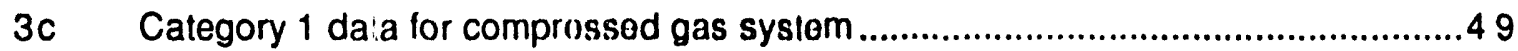

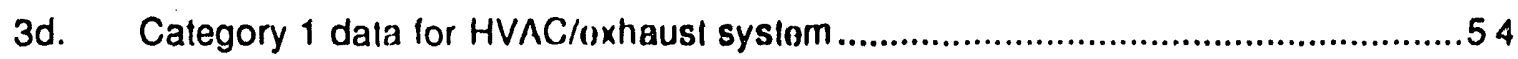

3e. Category 1 dala for electrical distribulion system ......................................60

3f. Category 1 data for instrumentation and control system ................................65 


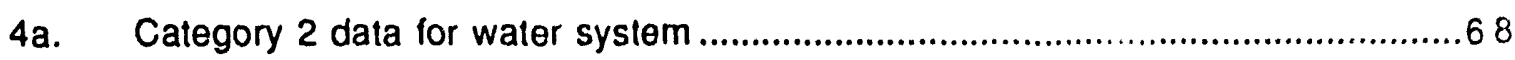

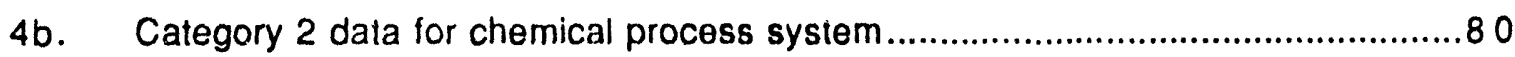

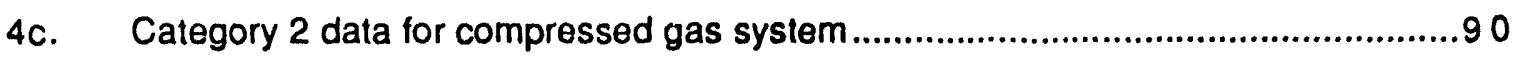

4d. Category 2 data for HVAC/exhaust system ...................................................100

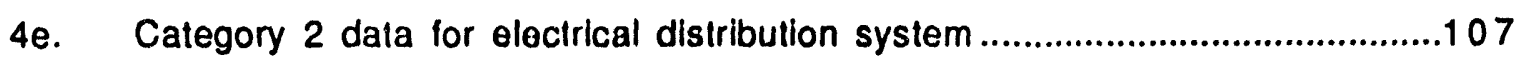

4f. Category 2 data for instrumbntallon and control system ................................113

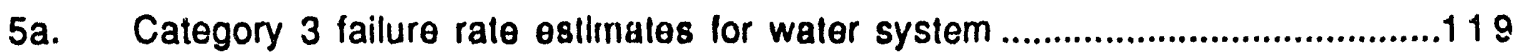

5b. Category 3 failure rate estimates for chenical process system.......................127

5c. Category 3 failure rate estimates for compressed gas system .......................134

5d. Category 3 failure rate estimales lor HVAC/exhaust system...........................140

5e. Category 3 failure rate ostimatos lor electrical distribution system ..............145

5f. Category 3 failure rate eslimales for instr. and control system ......................149

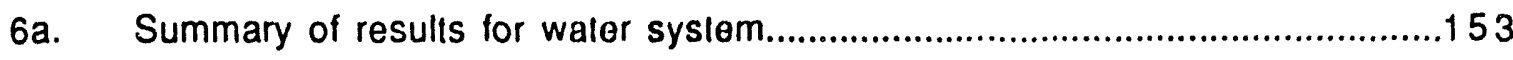

6b. Summary of results for chemical process system .........................................161

6c. Summary of results for compressed gas system ........................................ 69

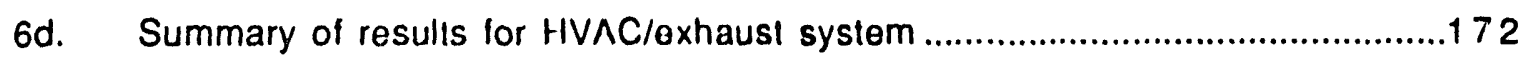

6e. Summary of rosulls lot uluctrical distribution system..................................182

6f. Summary of resulls for insirumontation and control system.........................186

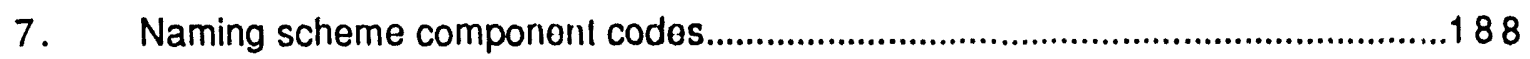

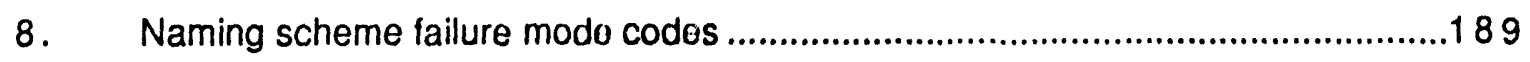

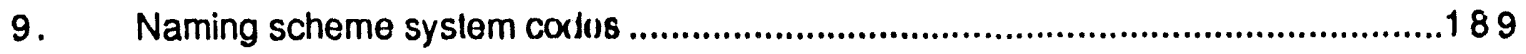


WSRC-TR-93-262

This Page Intentionally Left Blank 


\section{INTRODUCTION}

As part of an overall effort to upgrade the methodologies for safety analyses of nonreactor nuclear facilities at the Savannah River Site (SRS), a new generic component failure data base was developed. The project to update the SRS generic component failure data base had several goals:

Provide information for various failure modes for each component, where possible. An example is to provide failure rates for valve failure to open/close upon demand and valve spurious operation, rather than just valve failure. Such failure mode information is often necessary for detailed fault tree models.

- Base component failure rates on actual data (failure events) wherever possible.

Use the most up-to-date and applicable data sources available.

Provide a clear documentation trail for each component failure rate estimate, such that others can review the basis for each estimate.

The entire project was structured such that these goals could be accomplished. All of the goals were met.

Based on a review of existing safety analyses and component failure data bases, and based on additional input from SRS safety analysts, a comprehensive list of components and failure modes was generated. The list is shown in Table 1. Approximately 500 component and failure mode combinations were identified. Given this list of components and failure modes, sources were identified that contained applicable failure data or failure rate estimates. 


\section{DATA SOURCES}

Sources used to develop the SRS component generic data base may contain actual failure data or failure rate estimates. Also, sources containing failure data may vary in the quality or detail of the information. Therefore, three categories of sources were established:

Category 1 - Sources with actual failure data obtained from a detailed review of failure events (to ensure applicability to the failure mode being considered) and a detailed review of component populations and exposure durations (or demands). Such data include the plant-specific component failure data collected for probabilistic risk assessments (PRAs) or reliability studies. The NUCLARR ${ }^{1}$ data base has 19 Category 1 sources, all from nuclear power plants. For this project, the Savannah River Site (SRS) reactor data were added. The data sources include the following:

$$
\begin{aligned}
& \text { WSRC-RP-89-776, Rev. } 0^{3} \\
& \text { WSRC-RP-90-1258, Rev. } 0^{4} \\
& \text { DPST-87-642. }
\end{aligned}
$$

Including these Category 1 sources increased the Category 1 data by nearly $20 \%$.

Category 2 - Sources with actual failure data, but which have an added uncertainty in the data compared with Category 1 sources. This added uncertainty can result from a less comprehensive search for actual failures, a more approximate method for determining component populations or exposure durations (or demands), or a less clear breakdown of failures into the failuie modes of concern. NUCLARR has 10 Category 2 sources in its data base. For this project, an additional five Category 2 sources were identified and used:

NPRD $-3^{6}$ - By carefully reviewing failure mode information presented in tables in this document, the failure data presented in NPRD-3 could be divided into the failure modes of interest for this project.

OREDA $^{7}$. Failure data from components in offshore oil drilling facilities are presented in this report. In some cases judgment was needed to break down the data by failure mode.

- WIN-330 - This document lists failure data obtained from the Department of Energy (DOE) Idaho Chemical Processing Plant. However, this document does not distinguish failure modes, so some judgement was needed to break down the data by failure mode.

Tritium 8,9 , and 10 . These documents include failure data from a tritium handling facility at the Los Alamos National Laboratory. Again, judgement was needed to break down the data by failure mode. 
LNG111. Failure data from liquid natural ga. cility Cc: ponents are presented in this document. Judgement was needed to break down the data by failure mode.

Addition of these five sources more than doubled the amount of data from Category 2 sources in NUCLARR.

Category 3 - Sources that list only failure rate estimates. Six representative sources were used in this project. Many more such sources exit. The six were chosen to represent a variety of industries.

All of the sources used in this project are listed in Table 2. 


\section{AGGREGATION ROUTINES}

As discussed in Section 2 of this report, sources of data were grouped into three categories, depending upon the type and quality of information presented. For a given component and failure mode, several sources within a category might have failure information. Also, a single source may contain several sets of failure data. Therefore, aggregation routines are needed to combine the information from the different sources to obtain a representative result. Two different aggregation routines were used:

1. Combining actual failure data (failures and component exposure hours or demands) from different sources (used lor Categories 1 and 2)

2. Combining failure rate estimates that also include error factor estimates (used for Category 3).

For Categories 1 and 2 , the aggregation procedure is as follows ${ }^{1}$ :

1. Compute $R$ and $R_{2}$ from source data:

$$
\begin{aligned}
& R=\left(\Sigma f_{i}\right) /\left(\Sigma T_{i}\right) \\
& R_{2}=\left[\Sigma f_{i}\left(f_{i}-1\right) / T_{i}\right] /\left(\Sigma T_{i}\right)
\end{aligned}
$$

where $f_{i}=$ number of failures from source $i$

$$
T_{i}=\text { component exposure time }(h) \text { from source } i \text {. }
$$

2. Match moments to obtain underlying normal distribution:

$$
\begin{aligned}
& M=2 \ln (R)-0.5 \ln \left(R_{2}\right) \\
& S^{2}=\ln \left(R_{2}\right)-2 \ln (R) .
\end{aligned}
$$

3. Determine mean and error factor of lognormal distribution:

$$
\begin{aligned}
& M n=\exp \left(M+S^{2} / 2\right) \\
& E F=\exp (1.645 S)
\end{aligned}
$$

where $\mathrm{Mn}=$ mean

$$
E F=\text { error factor (95th percentile/50th percentile) } .
$$

For data involving demands rather than exposure hours, the same equations apply, but with $T_{i}$ replaced by $D_{i}$ (number of demands from source $i$ ). If the procedure described above resulted in an error factor greater than 30 , the error factor was chosen to be 30 . 
Three special casos may arise when aggregating actual failure data, such that the above routine does not work. The first is the case where only one data set is available (number of failures and number of hours or demands). In this case a Bayesian update of a non-informative prior was used to determine the mean estimate ${ }^{36}$ :

$$
\begin{array}{ll}
M n=(2 n+1) /(2 T) & \text { (for time-related data) } \\
M n=(2 n+1) /(2 D+2) & \text { (for demand-related data). }
\end{array}
$$

In both cases, an error factor of 10 was used. The error factor of 10 was chosen by examining the aggregated results in Tables 3 and 4 and calculating an average error factor.

The second special case occurs when all of the data sources involve zero or one failure. In this case, the data were combined (failures added and exposure hours or demands added) to obtain one data set which was then treated similarly to the case just described.

Finally, the third case arises when $S^{2}$ is less than zero. This case was treated the same as the second special case.

For Category 3, the aggregation routine is the following 37 :

1. Determine variance for each source:

$$
S_{i}^{2}=\left[\left(\operatorname{lnEF} F_{i}\right) / 1.645\right]^{2} \text {. }
$$

2. Determine natural logarithm of median for each source:

$$
\omega_{i}=\ln \left(M n_{i}\right)-s_{i}^{2} / 2
$$

where $\omega_{i}=$ natural logarithm of median.

3. Determine average of source variances:

$$
S_{\text {ave }}^{2}=\left(\Sigma s_{i}^{2}\right) / n_{s}
$$

where $n_{S}=$ number of sources.

4. Determine average of natural logarithms of medians:

$$
\omega_{\text {ave }}=\left(\Sigma \omega_{i}\right) / n_{s} \text {. }
$$

5. Determine variance of natural logarithms of medians:

$$
S_{\omega}^{2}=\Sigma\left(\omega_{i}-\omega_{\text {ave }}\right)^{2 / n_{s}}
$$


6. Determine mean and error factor of lognormal distribution:

$$
\begin{aligned}
& \omega=\left[\Sigma\left(\omega_{i} / S_{i}\right) /\left[\Sigma\left(1 / S_{i}\right)\right]\right] \\
& M_{n}=\exp \left(\omega+0.5 S^{2}\right) \\
& E F=\exp (1.645 S)
\end{aligned}
$$

where $S=$ larger of $S_{\text {ave }}$ or $S_{\omega}$.

Some of the Category 3 sources do not list error factors. In such cases, an error factor $(3,5,10$, or 30$)$ was assumed based on an average of error factors from the other sources. If no sources listed an error factor, then 10 was used. Also, if the aggregation resulted in an error factor greater than 30, then the error factor was assumed to be 30 . Finally, if a single source listed several failure rate estimates, then the estimates for that source were aggregated using the procedure described above. Then the result for that source was combined with the other sources.

Because a goal of this project was to base component failure rate estimates on actual failure data (contained in Categories 1 and 2) wherever possible, and because the quality of data in Category 1 sources is expected to be better than that for Category 2, aggregations were performed only within categories. Results from each category aggregation were not combined to obtain an overall result. 


\section{DATA SEARCH}

Results of the search for componenl failure information are summarized in Tables 3 through 5 . Tables $3 a$ through 31 prosent the Category 1 data by component, failure mode, and type of system. The NUCLARR1 column in Tables 3a through $3 f$ actually represents the aggregation of all of the Category 1 sources in NUCLARR. Tables $4 \mathrm{a}$ through $4 f$ present the Category 2 results, and Tables $5 \mathrm{a}$ through $5 \mathrm{f}$, the Category 3 results. Also presented in each table are the aggregated results (mean failure rate and error factor). 


\section{RECOMMENDED RESULTS}

Recommended component generic lailure rates are presented in Tables 1a through 1f. The recommended results were obtained trom the Category 1 aggregation, if such data existed. If not, then the Category 2 results were used. Finally, if neither Category 1 nor Category 2 information were available, then the Category 3 results were used. Also, because the product of this effort is a generic data base, the mean frequencies were rounded off to 1,3 , or 5 times the appropriate power of 10 . Error factors obtained from the aggregation process were rounded ofl to $3,5,10$, or 30 . Tables 6 a through $6 f$ contain more detailed notes concerning the selections of recommended failure rates.

A review of Tables 6 a through $6 f$ indicates that approximately $75 \%$ of the recommended failure rate estimates are based on actual failures from nuclear power plants or other types of facilities. The remaining $25 \%$ of the estimates had to be generated from sources listing only failurn rales.

It should be noted that failure dala may be reflective of a particular application or environment. In cases where data is limiled to sources of specialized application or operating environments, it may be appropriale to modify the recommended mean to account for alternative conditions. In all cases, engineering judgment should be used in the application of generic data.

Also lisied in Tables 1a through if are identifiers such as PIP.LE-W for each of the system/component/failure mode failuro rate distributions. In the identifier, the first three letters, PIP, indicate the type of component, which is a pipe. The second set of letters, LE, indicates the failure mode, which is external leakage. The final letter, W, indicates the type of system, which is walor system. Tables 7,8 , and 9 list the codes used for components, failure modes, and procossing fluids. Parts of this naming scheme were obtained from the report Review and Dovelopment of Common Nomenclature for Naming and Labeling Schemes for Probabilislic Bisk Assessment. 38 However, in some cases the naming schemes for components and failure modes in Reference 38 were changed to allow for a more consistent approach. Also, many additional components had to be added. 


\section{REFERENCES}

1. D. I. Gertman et al., Nuclear Compulerized Library for Assessing Reactor Beliability (NUCLARB), NUREG/CR-4639, June 1989.

2. J. N. Wilkinson et al., Idahe Chemical Processing Plant Failure Rate Database, Idaho National Engineering Laboratory, WIN-330, October 1991.

3. D. S. Cramer, Valve Reliability for the Level 1 PRA (U), Savannah River Site, WSRC-RP-89-776, Rev. 0, 1991.

4. D. S. Cramer, Check Valve Reliability for the Level 1 PRA (U), Savannah River Site, WSRC-RP-90-1258, Rev. 0, 1991.

5. D. S. Cramer, Data Base Developmenl and Equipment Reliability for Phase 1 of the Probabilistic Risk Analysis, Savannah River Site, DPST-87-642, October 1987.

6. M. J. Rossi, Nenelectronic Parts Reliability Data, Reliability Analysis Center, Rome Air Development Center, NPRD-3, 1985.

7. Offshore Reliability Dala Handbeek. Ollshore Reliability Data (OREDA), Norway, 1984 .

8. L. C. Cadwallader ánd M. A. Stolpe Gavoll, Iritium Waste Treatment System Component Failure Data Analysis from June 18. 1984 to December 31. 1989, Idaho National Engineering Laboratory, EGG-FSP-8973, Rev. 1, November 1990.

9. L. C. Cadwallader et al., Irilium Reem Air Moniter Component Failure Data Analysis from January 1. 1984 le Decomber 31.1990, Idaho National Engineering Laboratory, EGG-FSP-9450, May 1991.

10. L. C. Cadwallader and D. P. Sanchez, Socendary Containment System Component Failure Data Analysis from 1984 to 1991, Idaho National Engineering Laboratory, EGG-FSP-10323, Augusi 1992.

11. D. W. Johnson and J. R. Welker, Development of an Improved LNG Plant Failure Rate Data Base, Gas Research Institule, PB82-153503, September 1981.

12. Bia Bock Point Probabilistic Risk Assessment, Consumers Power Company, March 1981.

13. Oconee-3 PRA: A Probabilislic Risk $\triangle$ ssossment of Oconee Unit 3, Nuclear Safety Anctyss Center, Electric Power Rosuarch Institute, NSAC-60, June 1984.

14. Zion Probabilistic Safety Sludy, Commonwealth Edison Company, September 1981. 
15. Indian Point Probabilistic Safety Study, Pickard, Lowe, \& Garrick, Inc., December 1982.

16. J. P. Bento et al., Beliability Data Book for Compenents in Swedish Nuclear Eower Plants, Nuclear Safety Board of Swedish Utilities, RKS 85-25, 1985.

17. Millstene 1 Probabilistic Safety Study, Northeast Utilities, July 1985.

18. Connecticut Yankee Probabilistic Safety Study, Northeast Utilities, NUSCO-149, February 1986.

19. M. Trojovsky, Data Summaries of Licensee Event Reports of Pumps at U.S. Commercial Nuclear Power Plants: January 1. 1972 to September 30. 1980. NUREG/CR-1205, Rev. 1, January, 1982.

20. C. F. Miller et al., Data Summaries of Licensee Event Reponts of Valves at U.S. Commercial Nuclear Power Plants, NUREG/CR-1363, Rev. 1, October 1982.

21. M. Trojovsky and S. Brown, Data Summaries of Licensee Event Reports of Inverters at U.S. Commercial Nuclear Power Plants, NUREG/CR-3867, August 1984.

22. C. F. Miller et al., Data Summaries of Licensee Event Reports of Selected Instrumentation and Control Components at U.S. Commercial Nuclear Power Plants, NUREG/CR-1740, May 1981.

23. J. P. Drago et al., The In-Plant Reliability Data Base for Nuclear Plant Components: Interim Data Report - The PUmp Component, NUREG/CR-2886, December 1982.

24. R. J. Borkowski et al., The In-Plant Reliability Data Base for Nuclear Plant Components: Interim Data Repert. The Valve Component, NUREG/CR-3154, December 1983.

25. W. K. Kahl and R. J. Borkowski, The In-Plant Reliability Data Base for Nuclear Plant Components: Interim Report-Diesel Generators. Batteries, Chargers, and Inverters, NUREG/CR-3831, January 1985.

26. H. Wykoff, The Reliability of Emergency Diesel Generators at U. S. Nuclear Power Plants, Nuclear Safety Analysis Center, Electric Power Research Insiitute, NSAC/108, September 1986.

27. R. E. Wright et al., Pipe Break Frequency Estimation for Nuclear Power Plants, NUREG/CR-4407, May 1987.

28. S. A. Eide et al., Component External Leakage and Ruplure Frequency Estimates, Idaho National Engineering Laboratory, EGG-SSRE-9639, November 1991.

29. Reacter Safety Study: An Assessment of Accident Risks in U. S. Commercial Nuclear Power Plants, WASH-1400 (NUREG 75/014), October 1975. 
30. Guidelines for Process Equipment Reliability Data With Data Tables, Center for Chemical Process Safety, American Institute of Chemical Engineers, 1989.

31. IEEE Guide to the Collection and Presentation of Electrical. Electronic. Sensine Component and Mechanical Equipment Reliability Data for Nuclear-Power Generating Stations, Institute of Electrical and Electronic Engineers, Inc., IEEE Std 500-1984, 1983.

32. H. Lammerse and M. Bosman, "Data of Gas Compressors and Instrumentation Hard to Collect, Easy to Analyse," Reliability Engineering, 13 (1985), pp. 6578 .

33. R. P. Dawkins and J. A. Derdiger, Compenent Failure and Repair Data: Gasification-Combined-Cycle Power Generation Units, Electric Power Research Institute, Topical Report AP-2205, Research Project 239-2, February 1982.

34. J. A. Derdiger et al., Compenent Failure and Repair Data for Coal-Fired Power Units, Electric Power Research Institute, Topical Report AP-2071, Research Project 239-2, October 1981.

35. S. A. Eide et al., Generic Component Failure Data Base for Light Water and Liquid Sodium Reactor PRAs, Idaho National Engineering Laboratory, EGG-SSRE-8875, February 1990.

36. PRA Procedures Guide, U. S. Nuclear Regulatory Commission, NUREG/CR-2300, January 1983.

37. H. F. Martz and M. C. Bryson, "On Combining Data for Estimating the Frequency of Low-Probability Events with Application to Sodium Valve Failure Rates," Nuclear Science and Engineering, 83 (1983), pp. 267-280.

38. A. D. Trusty and D. P. Mackowiak, Review and Development of Common Nemenclature for Naming and Labeling Schemes for Probabilistic Risk Assessment, U. S. Nuclear Regulatory Commission, NUREG/CR-5905, August 1992. 


\begin{tabular}{|c|c|c|c|}
\hline \multirow[t]{2}{*}{ System/Component/Failure Mode } & \multirow[t]{2}{*}{ Identifier } & \multicolumn{2}{|c|}{$\begin{array}{l}\text { Recommrended Failure Rate } \\
\text { Distribution (lognormal) }\end{array}$} \\
\hline & & Mean & Error Factor ${ }^{a}$ \\
\hline $\begin{array}{l}\text { Water } \\
\text { Valve (Standby or Safety) } \\
\text { Manual } \\
\text { Fails to open/close }\end{array}$ & $\begin{array}{l}\text { XVM-CC-W } \\
\text { or } \\
\text { XVM-OO-W }\end{array}$ & $3.0 \mathrm{E}-4 / \mathrm{d}$ & 10 \\
\hline Plugs & XVM-PG-W & $5.0 \mathrm{E}-8 / \mathrm{h}$ & 10 \\
\hline Leakage (internal) & XVM-LI-W & $1.0 \mathrm{E}-6 / \mathrm{h}$ & 10 \\
\hline Rupture (internal) & XVM-RI-W & $5.0 \mathrm{E}-8 / \mathrm{h}$ & 30 \\
\hline Leakage (external) & XVM-LE-W & $1.0 \mathrm{E}-8 / \mathrm{h}$ & 10 \\
\hline Rupture (external) & XVM-RE-W & $5.0 \mathrm{E}-10 / \mathrm{h}$ & 30 \\
\hline $\begin{array}{l}\text { Check } \\
\text { Fails to open }\end{array}$ & CKV-CC-W & $5.0 \mathrm{E}-5 / \mathrm{d}$ & 10 \\
\hline Fails to close & CKV-00-W & $1.0 \mathrm{E}-3 / \mathrm{d}$ & 5 \\
\hline Plugs & CKV-PG-W & $5.0 \mathrm{E}-8 / \mathrm{h}$ & 10 \\
\hline Leakage (internal) & CKV-LI.W & $1.0 \mathrm{E}-6 / \mathrm{h}$ & 10 \\
\hline Rupture (internal) & CKV-RI-W & $5.0 \mathrm{E}-8 / \mathrm{h}$ & 30 \\
\hline Leakage (external) & CKV-LE-W & $1.0 \mathrm{E}-8 / \mathrm{h}$ & 10 \\
\hline Rupture (external) & CKV-RE-W & $5.0 \mathrm{E}-10 / \mathrm{h}$ & 30 \\
\hline $\begin{array}{l}\text { Motor-Operated } \\
\text { Fails to open/close }\end{array}$ & $\begin{array}{l}\text { MOV-CC-W } \\
\text { or } \\
\text { MOV-OO-W }\end{array}$ & $3.0 \mathrm{E}-3 / \mathrm{d}$ & 5 \\
\hline Spurious operation & $\begin{array}{l}\text { MOV-CO-W } \\
\text { or } \\
\text { MOV-OC-W }\end{array}$ & $3.0 \mathrm{E}-7 / \mathrm{h}$ & 5 \\
\hline Plugs & MOV-PG-W & $5.0 \mathrm{E}-8 / \mathrm{h}$ & 10 \\
\hline Leakage (internal) & MOV-LI-W & $1.0 \mathrm{E}-6 / \mathrm{h}$ & 10 \\
\hline Rupture (internal) & MOV-RI-W & $5.0 \mathrm{E}-8 / \mathrm{h}$ & 30 \\
\hline Leakage (external) & MOV-LE-W & $1.0 \mathrm{E}-8 / \mathrm{h}$ & 10 \\
\hline Rupture (external) & MOV-RE-W & $5.0 \mathrm{E}-10 / \mathrm{h}$ & 30 \\
\hline $\begin{array}{l}\text { Air-Operated } \\
\text { Fails to open/close }\end{array}$ & $\begin{array}{l}\text { AOV-CC-W } \\
\text { or } \\
\text { AOV-OO-W }\end{array}$ & $1.0 \mathrm{E}-3 / \mathrm{d}$ & 30 \\
\hline Spurious operation & $\begin{array}{l}\text { AOV-CO-W } \\
\text { or } \\
\text { AOV-OC-W }\end{array}$ & $1.0 \mathrm{E}-6 / \mathrm{h}$ & 5 \\
\hline Plugs & AOV-PG-W & $5.0 \mathrm{E}-8 / \mathrm{h}$ & 10 \\
\hline Leakage (intemal) & AOV-LI-W & $1.0 \mathrm{E}-6 / \mathrm{h}$ & 10 \\
\hline Rupture (internal) & AOV-RI-W & $5.0 \mathrm{E}-8 / \mathrm{h}$ & 30 \\
\hline Leakage (external) & AOV-LE-W & $1.0 \mathrm{E}-8 / \mathrm{h}$ & 10 \\
\hline Rupture (external) & AOV-RE-W & $5.0 \mathrm{E}-10 / \mathrm{h}$ & 30 \\
\hline $\begin{array}{l}\text { Solenoid-Operated } \\
\text { Fails to open/close }\end{array}$ & $\begin{array}{l}\text { SOV-CC-W } \\
\text { or } \\
\text { SOV-OO-W }\end{array}$ & $1.0 \mathrm{E}-3 / \mathrm{d}$ & 10 \\
\hline Spurious operation & $\begin{array}{l}\text { SOV-CO-W } \\
\text { or } \\
\text { SOV-OC-W } \\
\end{array}$ & $5.0 \mathrm{E}-7 / \mathrm{h}$ & 10 \\
\hline
\end{tabular}

Note: Modification of the recommended mean for hourly failure rates may be appropriate for components subjected to hostile radioactive or chemical environments. 
WSRC-TR-93-262

\begin{tabular}{|c|c|c|c|}
\hline \multirow[t]{2}{*}{ System/Componen/Failure Mode } & \multirow[t]{2}{*}{ Identifier } & \multicolumn{2}{|c|}{$\begin{array}{l}\text { Recommended Failure Rate } \\
\text { Distribution (lognormal) }\end{array}$} \\
\hline & & Mean & Error Factor \\
\hline Plugs & SOV-PG-W & $5.0 \mathrm{E}-8 / \mathrm{h}$ & 10 \\
\hline Leakage (internal) & SOV-LI-W & $1.0 \mathrm{E}-6 / \mathrm{h}$ & 10 \\
\hline Rupture (internal) & SOV-RI-W & $5.0 \mathrm{E} \cdot 8 / \mathrm{h}$ & 30 \\
\hline Leakage (external) & SOY-LE-W & $1.0 \mathrm{E}-8 / \mathrm{h}$ & 10 \\
\hline Rupture (external) & $\overline{S O V}-\mathrm{RE}-\mathrm{W}$ & $5.0 \mathrm{E}-10 / \mathrm{h}$ & 30 \\
\hline $\begin{array}{l}\text { Safety/Relief } \\
\text { Fails to open } \\
\end{array}$ & SRV-CC-W & $3.0 \mathrm{E}-3 / \mathrm{d}$ & 3 \\
\hline Fails to reclose & SRY-OO-W & $3.0 \mathrm{E}-3 / \mathrm{d}$ & 3 \\
\hline Leakage (internal) & SRV-LI-W & $1.0 \mathrm{E}-6 / \mathrm{h}$ & 10 \\
\hline Rupture (internal) & SRV-RI-W & $5.0 \mathrm{E}-8 / \mathrm{h}$ & 30 \\
\hline Leakage (external) & SRV-LE-W & $1.0 \mathrm{E}-8 / \mathrm{h}$ & 10 \\
\hline Ruplure (external) & SRV-RE-W & $5.0 \mathrm{E}-10 / \mathrm{h}$ & 30 \\
\hline $\begin{array}{c}\text { Vacue Breaker } \\
\text { Fin in open } \\
\end{array}$ & VBV-CC.W & $1.0 \mathrm{E} \cdot 2 / \mathrm{d}$ & 10 \\
\hline tails to reclose & $V B V \cdot(X) \cdot W$ & $1.0 \mathrm{E}-2 / \mathrm{d}$ & 10 \\
\hline Leakage (internal) & VBV-LI-W & $1.0 \mathrm{E}-6 / \mathrm{h}$ & 10 \\
\hline Rupture (intemal) & VBV-RI-W & $5.0 \mathrm{E}-8 / \mathrm{h}$ & 30 \\
\hline Leakage (exi rnal) & VBV-LE-W & $1.0 \mathrm{E}-8 / \mathrm{h}$ & 10 \\
\hline Rupture (e): rnal) & VBV-RE-W & $5.0 \mathrm{E}-10 / \mathrm{h}$ & 30 \\
\hline $\begin{array}{l}\text { Explosive } \\
\text { Fails to open }\end{array}$ & EXV-CC.W & $1.0 \mathrm{E}-4 / \mathrm{d}$ & 10 \\
\hline Leakage (internal) & EXV-LI-W & $1.0 \mathrm{E}-6 / \mathrm{h}$ & 10 \\
\hline Rupture (internal) & EXV-RI-W & $5.0 \mathrm{E} \cdot 8 / \mathrm{h}$ & 30 \\
\hline Leakage (external) & EXV-LE-W & $1.0 \mathrm{E}-8 / \mathrm{h}$ & 10 \\
\hline Rupture (external) & EXV-RE-W & $5.0 \mathrm{E}-10 / \mathrm{h}$ & 30 \\
\hline $\begin{array}{l}\text { Valve (Control) } \\
\text { Molor-Operated } \\
\text { Fails open }\end{array}$ & CMV-FO-W & $3.0 \mathrm{E}-6 / \mathrm{h}$ & 10 \\
\hline Fails closed & CMV-FC-W & $3.0 \mathrm{E}-6 / \mathrm{h}$ & 10 \\
\hline Fails to respond & CMV-NR-W & $3.0 \mathrm{E} \cdot 6 / \mathrm{h}$ & 10 \\
\hline Plugs & CMV-PG-W & $5.0 \mathrm{E}-8 / \mathrm{h}$ & 10 \\
\hline Leakage (external) & CMV-LE-W & $1.0 \mathrm{E} \cdot 8 / \mathrm{h}$ & 10 \\
\hline Rupture (external) & CMV-RE-W & $5.0 \mathrm{E} \cdot 10 / \mathrm{h}$ & 30 \\
\hline $\begin{array}{l}\text { Air-Operated } \\
\text { Fails open }\end{array}$ & CAV-FO-W & $3.0 \mathrm{E}-6 / \mathrm{h}$ & 10 \\
\hline Fails closed & CAV-FC.W & $3.0 \mathrm{E}-6 / \mathrm{h}$ & 10 \\
\hline Fails to respond & CAV-NR-W & $3.0 \mathrm{E}-6 / \mathrm{h}$ & 10 \\
\hline Plugs & CAV-PG.W & $5.0 \mathrm{E}-8 / \mathrm{h}$ & 10 \\
\hline Leakage (external) & CAV-LEWW & $1.0 \mathrm{E} \cdot 8 / \mathrm{h}$ & 10 \\
\hline R.jiture (exterta!) & CAVPPW & $50 \mathrm{E} 10 / \mathrm{h}$ & 30 \\
\hline
\end{tabular}

- Note: Modification of the recommended mean for hourly failure rates may be appropriate for components subjected to hostile radioactive or chemical environments. 


\begin{tabular}{|c|c|c|c|}
\hline \multirow[t]{2}{*}{ System/Component/Failure Mode } & \multirow[t]{2}{*}{ Identifier } & \multicolumn{2}{|c|}{$\begin{array}{l}\text { Recommended Failure Rate } \\
\text { Distribution (lognormal) }\end{array}$} \\
\hline & & Mean & Error Factor ${ }^{a}$ \\
\hline $\begin{array}{l}\text { Solenoid-Operated } \\
\text { Fails open }\end{array}$ & $\overline{\text { CSV-FO-W }}$ & $3.0 \mathrm{E}-6 / \mathrm{h}$ & 10 \\
\hline Fails closed & CSV-FC-W & $3.0 \mathrm{E}-6 / \mathrm{h}$ & 10 \\
\hline Fails to respond & CSV-FO-W & $3.0 \mathrm{E}-6 / \mathrm{h}$ & 10 \\
\hline Plugs & CSV-PG-W & $5.0 \mathrm{E}-8 / \mathrm{h}$ & 10 \\
\hline Leakage (external) & CSV-LE-W & $1.0 \mathrm{E}-8 / \mathrm{h}$ & 10 \\
\hline Rupture (external) & CSV-RE-W & $5.0 \mathrm{E}-10 / \mathrm{h}$ & 30 \\
\hline $\begin{array}{l}\text { Pump } \\
\text { Motor-Driven } \\
\text { Fails to start }\end{array}$ & MDP-FS-W & $3.0 \mathrm{E}-3 / \mathrm{d}$ & $\overline{5}$ \\
\hline Fails to nun & MDP-FR-W & $3.0 \mathrm{E}-5 / \mathrm{h}$ & 10 \\
\hline Overspeed & MDP-OS-W & $5.0 \mathrm{E}-6 / \mathrm{h}$ & 10 \\
\hline Fails to stop & MDP-NS-W & $3.0 \mathrm{E}-3 / \mathrm{d}$ & 5 \\
\hline Leakage (external) & MDP.LE-W & $3.0 \mathrm{E}-8 / \mathrm{h}$ & 10 \\
\hline Rupture (external) & MDP-RE-W & $1.0 \mathrm{E}-9 / \mathrm{h}$ & 30 \\
\hline $\begin{array}{l}\text { Turbine-Driven } \\
\text { Fails to start }\end{array}$ & TDP-FS-W & $3.0 \mathrm{E}-2 / \mathrm{d}$ & 3 \\
\hline Fails to run & TDP-FR-W & $1.0 \mathrm{E}-4 / \mathrm{h}$ & 30 \\
\hline Overspeed & TDP-OS-W & $3.0 \mathrm{E}-5 / \mathrm{h}$ & 10 \\
\hline Fails to stop & TDP-NS-W & $3.0 \mathrm{E}-2 / \mathrm{d}$ & 3 \\
\hline Leakage (external) & TDP-LE-W & $3.0 \mathrm{E}-8 / \mathrm{h}$ & 10 \\
\hline Rupture (external) & TDP-RE-W & $1.0 \mathrm{E}-9 / \mathrm{h}$ & 30 \\
\hline $\begin{array}{l}\text { Diesel-Driven } \\
\text { Fails to start }\end{array}$ & DDP-FS-W & $1.0 \mathrm{E}-2 / \mathrm{d}$ & 5 \\
\hline Fails to run & DDP-FR.W & $5.0 \mathrm{E}-3 / \mathrm{h}$ & 5 \\
\hline Overspeed & DDP-OS-W & $1.0 \mathrm{E}-3 / \mathrm{h}$ & 5 \\
\hline Fails to stop & DDP-NS-W & $1.0 \mathrm{E}-2 / \mathrm{d}$ & 5 \\
\hline Leakage (external) & DDP-LE-W & $3.0 \mathrm{E}-8 / \mathrm{h}$ & 10 \\
\hline Rupture (external) & DDP-RE-W & $1.0 \mathrm{E}-9 / \mathrm{h}$ & 30 \\
\hline $\begin{array}{l}\text { Piping/Hose/Jumper } \\
\text { Piping } \\
\text { Leakage (external) }\end{array}$ & PIP-LE-W & $3.0 \mathrm{E}-9 / \mathrm{h}-\mathrm{ft}$ & 10 \\
\hline Rupture (external) & PIP-RE-W & $1.0 \mathrm{E}-10 / \mathrm{h}-\mathrm{ft}$ & 30 \\
\hline Plugs & PIP.PF-W & $1.0 \mathrm{E}-10 / \mathrm{h}-\mathrm{ft}$ & 30 \\
\hline $\begin{array}{l}\text { Hose } \\
\text { Leakage (external) }\end{array}$ & HOS-LE-W & $1.0 \mathrm{E}-9 / \mathrm{h}-\mathrm{ft}$ & 10 \\
\hline Rupture (external) & HOS-RE-W & $1.0 \mathrm{E}-8 / \mathrm{h}-\mathrm{ft}$ & 10 \\
\hline Plugs & HOS-PG-W & $1.0 \mathrm{E}-8 / \mathrm{h}-\mathrm{ft}$ & 10 \\
\hline $\begin{array}{l}\text { Jumper } \\
\text { Leakage (external) }\end{array}$ & JPR-LE-W & $1.0 \mathrm{E}-6 / \mathrm{h}$ & 10 \\
\hline
\end{tabular}

Note: Modification of the recommended mean for hourly failure rates may be appropriate for components subjected to hostile radioactive or chemical environments. 


\begin{tabular}{|c|c|c|c|}
\hline \multicolumn{4}{|c|}{ Table 1a. Woit system recommended generic failure rates (c } \\
\hline \multirow[t]{2}{*}{ System/Component/Failure Mode } & \multirow[t]{2}{*}{ Identifie: } & \multicolumn{2}{|c|}{$\begin{array}{l}\text { Reconnticnded Failure Rate } \\
\text { Distribution (lognormal) }\end{array}$} \\
\hline & & Mean & Error Factor ${ }^{2}$ \\
\hline Rupture (external) & JPR-RE-W & $1.0 \mathrm{E}-8 / \mathrm{h}$ & 30 \\
\hline Plugs & JPR-PG-W & $5.0 \mathrm{E}-8 / \mathrm{h}$ & 10 \\
\hline $\begin{array}{l}\text { Vessel } \\
\text { Tank (Unpressurized) } \\
\text { Leakage (external) }\end{array}$ & TKU-LE-W & $1.0 \mathrm{E}-8 / \mathrm{h}$ & 10 \\
\hline Rupture (external) & TKU-RE-W & $5.0 \mathrm{E}-10 / \mathrm{h}$ & 30 \\
\hline $\begin{array}{l}\text { Tank (Pressurized) } \\
\text { Leakage (external) }\end{array}$ & TKP-LE-W & $1.0 \mathrm{E}-8 / \mathrm{h}$ & 10 \\
\hline Rupture (external) & TKP-RE-W & $5.0 \mathrm{E} \cdot 10 / \mathrm{h}$ & 30 \\
\hline $\begin{array}{l}\text { Flange/Gasket } \\
\text { Leakage (external) }\end{array}$ & FLG-LE-W & $1.0 \mathrm{E}-\overline{8 / \mathrm{h}}$ & 10 \\
\hline Rupture (external) & FLG-RE-W & $1.0 \mathrm{E-10/ \textrm {h }}$ & 30 \\
\hline $\begin{array}{l}\text { Heat Exchanger } \\
\text { Shell/Tube } \\
\text { Fouling (tubes) }\end{array}$ & HTX-FL-W & $1.0 \mathrm{E}-7 / \mathrm{h}$ & 10 \\
\hline Plugs (tubes) & HTX.PG-W & $3.0 \mathrm{E} \cdot 8_{i i}$ & 10 \\
\hline Leakage (tubes) & IITX.LI.W & $1.11 \div 7 / \mathrm{h}$ & 10 \\
\hline Rupture (tubes) & HTX-RI-W & $5.0 \mathrm{E}-9 / \mathrm{h}$ & 30 \\
\hline Leakage (shell) & HTX-LE-W & $1.0 \mathrm{E}-8 / \mathrm{h}$ & 10 \\
\hline Rupture (shell) & HTX-RE-W & $5.0 \mathrm{E}-10 / \mathrm{h}$ & 30 \\
\hline $\begin{array}{l}\text { Heater (Electrical) } \\
\text { Fails to heat }\end{array}$ & HTE-FH-W & $1.0 \mathrm{E}-6 / \mathrm{h}$ & 10 \\
\hline Overheats & HTE-OH-W & $3.0 \mathrm{E}-7 / \mathrm{h}$ & 10 \\
\hline Leakage (external) & HTE-LE-W & $1.0 \mathrm{E}-7 / \mathrm{h}$ & 10 \\
\hline Rupture (external) & HTE-RE-W & $5.0 \mathrm{E}-9 / \mathrm{h}$ & 30 \\
\hline $\begin{array}{c}\text { Strainer/Filter } \\
\text { Plugs } \\
\end{array}$ & FLT-PG-W & $3.0 \mathrm{E}-6 / \mathrm{h}$ & 10 \\
\hline Leakage (internal) & FLT-LI-W & $3.0 \mathrm{E}-6 / \mathrm{h}$ & 10 \\
\hline Rupture (internal) & FLT-RI-W & $5.0 \mathrm{E}-7 / \mathrm{h}$ & 10 \\
\hline $\begin{array}{l}\text { Orifice } \\
\text { Plugs } \\
\end{array}$ & ORF-PG-W & $1.0 \mathrm{E}-6 / \mathrm{h}$ & 3 \\
\hline $\begin{array}{l}\text { Miscellaneous } \\
\text { Travelling Screen } \\
\text { Plugs }\end{array}$ & TRS-PG-W & $5.0 \mathrm{E}-7 / \mathrm{h}$ & 10 \\
\hline
\end{tabular}

a. Error factor is the 95 th percentile/50th percentile.

- Note: Modification of the recommended mean for hourly failure rates may be appropriate for components subjected to hostile radioactive or chemical environments. 


\begin{tabular}{|c|c|c|c|}
\hline \multirow[t]{2}{*}{ System/Component/Failure Mode } & \multirow[t]{2}{*}{ Identifier } & \multicolumn{2}{|c|}{$\begin{array}{l}\text { Recommended Failure Rate } \\
\text { Distribution (lognormal) }\end{array}$} \\
\hline & & Mean & Error Factor ${ }^{\mathrm{H}}$ \\
\hline $\begin{array}{l}\text { Chemical Process } \\
\text { Valve (Standby or Safety) } \\
\text { Manual } \\
\text { Fails to open/close }\end{array}$ & $\begin{array}{l}\text { XVM-CC-C or } \\
\text { XVM-OO-C }\end{array}$ & $3.0 \mathrm{E}-4 / \mathrm{d}$ & 10 \\
\hline Plugs & XVM-PG-C & $5.0 \mathrm{E}-8 / \mathrm{h}$ & 10 \\
\hline Leakage (intemal) & XVM-LI-C & $1.0 \mathrm{E}-6 / \mathrm{h}$ & 10 \\
\hline Rupture (intemal) & XVM-RI-C & $5.0 \mathrm{E}-8 / \mathrm{h}$ & 30 \\
\hline Leakage (external) & XVM-LE-C & $5.0 \mathrm{E}-7 / \mathrm{h}$ & 10 \\
\hline Rupture (external) & XVM-RE-C & $3.0 \mathrm{E}-8 / \mathrm{h}$ & 30 \\
\hline $\begin{array}{l}\text { Check } \\
\text { Fails to open }\end{array}$ & $\overline{\mathrm{CKV}}-\overline{\mathrm{CC}-\mathrm{C}}$ & $5.0 \mathrm{E}-5 / \mathrm{d}$ & 10 \\
\hline Fails to close & CKV-OO-C & $1.0 \mathrm{E}-3 / \mathrm{d}$ & 10 \\
\hline Plugs & CKV-PG-C & $5.0 \mathrm{E}-8 / \mathrm{h}$ & 10 \\
\hline Leakage (internal) & CKV-LI-C & $1.0 \mathrm{E}-6 / \mathrm{h}$ & 10 \\
\hline Rupture (intemal) & CKV-RI-C & $5.0 \mathrm{E}-8 / \mathrm{h}$ & 30 \\
\hline Leakage (external) & CKV-LE-C & $5.0 \mathrm{E}-7 / \mathrm{h}$ & 10 \\
\hline Rupture (external) & CKV-RE-C & $3.0 \mathrm{E}-8 / \mathrm{h}$ & 30 \\
\hline $\begin{array}{l}\text { Motor-Operated } \\
\text { Fails to open/close }\end{array}$ & $\begin{array}{l}\text { MOV-CC-C } \\
\text { or } \\
\text { MOV-OO-C }\end{array}$ & $3.0 \mathrm{E}-3 / \mathrm{d}$ & 10 \\
\hline Spurious operation & $\begin{array}{l}\text { MOV-CO-C } \\
\text { or } \\
\text { MOV-OC-C }\end{array}$ & $3.0 \mathrm{E}-7 / \mathrm{h}$ & 10 \\
\hline Plugs & MOV-PG-C & $5.0 \mathrm{E}-8 / \mathrm{h}$ & 10 \\
\hline Leakage (internal) & MOV-LI-C & $1.0 \mathrm{E}-6 / \mathrm{h}$ & 10 \\
\hline Rupture (internal) & MOV-RI-C & $5.0 \mathrm{E}-8 / \mathrm{h}$ & 10 \\
\hline Leakage (external) & MOV-LE-C & $5.0 \mathrm{E}-7 / \mathrm{h}$ & 10 \\
\hline Rupture (external) & MOV-RE-C & $3.0 \mathrm{E}-8 / \mathrm{h}$ & 30 \\
\hline $\begin{array}{l}\text { Air-Operated } \\
\text { Fails to open/close }\end{array}$ & $\begin{array}{l}\mathrm{AOV}-\mathrm{CC}-\mathrm{C} \\
\text { or } \\
\mathrm{AOV}-\mathrm{OO}-\mathrm{C}\end{array}$ & $1.0 \mathrm{E}-3 / \mathrm{d}$ & 30 \\
\hline Spurious operation & $\begin{array}{l}\text { AOV-CO-C } \\
\text { or } \\
\text { AOV-OC-C }\end{array}$ & $1.0 \mathrm{E}-6 / \mathrm{h}$ & 10 \\
\hline Plugs & $\begin{array}{l}\text { AVO-CO-C } \\
\text { or } \\
\text { AOV }-\mathrm{OC}-\mathrm{C}\end{array}$ & $5.0 \mathrm{E}-8 / \mathrm{h}$ & 10 \\
\hline Leakage (intemal) & AOV-LI-C & $1.0 \mathrm{E}-6 / \mathrm{h}$ & 10 \\
\hline Rupture (internal) & AOV-RI-C & $5.0 \mathrm{E}-8 / \mathrm{h}$ & 30 \\
\hline Leakage (external) & AOV-LE-C & $5.0 \mathrm{E}-7 / \mathrm{h}$ & 10 \\
\hline Rupture (external) & AOV-RE-C & $3.0 \mathrm{E}-8 / \mathrm{h}$ & 30 \\
\hline $\begin{array}{l}\text { Solenoid-Operated } \\
\text { Fails to open/close }\end{array}$ & $\begin{array}{l}\text { SOV-CC-C or } \\
\text { SOV-OO-C }\end{array}$ & $1.0 \mathrm{E}-3 / \mathrm{d}$ & 10 \\
\hline Spurious uperation & $\begin{array}{l}\text { SOV-CO-C } \\
\text { or } \\
\text { SOV-OC-C }\end{array}$ & $5.0 \mathrm{E}-7 / \mathrm{h}$ & 10 \\
\hline
\end{tabular}

Note: Modification of the recommended mean for hourly failure rates may be appropriatc for cornponents subjected to hostile radioactive or chemical environments. 
WSRC-TR-93-262

\begin{tabular}{|c|c|c|c|}
\hline \multirow[t]{2}{*}{ System/Component/Failure Mode } & \multirow[t]{2}{*}{ Identifier } & \multicolumn{2}{|c|}{$\begin{array}{l}\text { Recommended Failure Rate } \\
\text { Distribution (lognormal) }\end{array}$} \\
\hline & & Mean & Error Factor ${ }^{\mathrm{a}}$ \\
\hline Plugs & SOV-PG-C & $5.0 \mathrm{E}-8 / \mathrm{h}$ & 10 \\
\hline Leakage (internal) & SOV-LI-C & $1.0 \mathrm{E}-6 / \mathrm{h}$ & 10 \\
\hline Rupture (intemal) & SOV-RI-C & $5.0 \mathrm{E}-8 / \mathrm{h}$ & 30 \\
\hline Leakage (external) & SOV-LE-C & $5.0 \mathrm{E}-7 / \mathrm{h}$ & 10 \\
\hline Rupture (external) & SOV-RE-C & $3.0 \mathrm{E}-8 / \mathrm{h}$ & 30 \\
\hline $\begin{array}{l}\text { Safety/Relief } \\
\text { Fails to open }\end{array}$ & SRV-CC-C & $3.0 \mathrm{E}-3 / \mathrm{d}$ & 10 \\
\hline Fails to reclose & SRV-OOC & $3.0 \mathrm{E}-3 / \mathrm{d}$ & 10 \\
\hline Leakage (internal) & SRV-LI-C & $1.0 \mathrm{E}-6 / \mathrm{h}$ & 10 \\
\hline Rupture (intemal) & SRV-RI-: & $5.0 \mathrm{E}-8 / \mathrm{h}$ & 30 \\
\hline Leakage (external) & SRV-LE-C & $5.0 \mathrm{E}-7 / \mathrm{h}$ & 10 \\
\hline Rupture (external) & SRV-RE-C & $3.0 \mathrm{E}-8 / \mathrm{h}$ & 30 \\
\hline $\begin{array}{l}\text { Vacuum-Breaker } \\
\text { Fails to open }\end{array}$ & $\overline{V B V-C C}-\bar{C}$ & $1.0 \mathrm{E}-2 / \mathrm{d}$ & 10 \\
\hline Fails to reclose & VBV-OOCC & $1.0 \mathrm{E}-2 / \mathrm{d}$ & 10 \\
\hline Leakage (intemal) & VBV-LI-C & $\overline{1.0 \mathrm{E}-6 / \mathrm{il}}$ & 10 \\
\hline Rupture (internal) & VBV-RI-C & $5.0 \mathrm{E}-8 / \mathrm{h}$ & 30 \\
\hline Leakage (external) & VBV-LE-C & $5.0 \mathrm{E}-7 / \mathrm{h}$ & 10 \\
\hline Rupture (external) & VBV-RE-C & $3.0 \mathrm{E}-8 / \mathrm{h}$ & 30 \\
\hline $\begin{array}{l}\text { Explosive } \\
\text { Fails to open }\end{array}$ & $\overline{\text { EXV-CC-C }}$ & $1.0 \mathrm{E}-4 / \mathrm{d}$ & 10 \\
\hline Leakage (internal) & EXV-LI-C & $1.0 \mathrm{E}-6 / \mathrm{h}$ & 10 \\
\hline Rupture (internal) & EXV-RI-C & $5.0 \mathrm{E}-8 / \mathrm{h}$ & 10 \\
\hline Leakage (external) & EXV-LE-C & $5.0 \mathrm{E}-7 / \mathrm{h}$ & 10 \\
\hline Rupture (external) & EXV-RE-C & $3.0 \mathrm{E}-8 / \mathrm{h}$ & 30 \\
\hline $\begin{array}{c}\text { Valve (Control) } \\
\text { Motor-Operated } \\
\text { Fails open } \\
\end{array}$ & CMV-FO-C & $3.0 \mathrm{E}-6 / \mathrm{h}$ & 10 \\
\hline Fails closed & $\overline{C M V-F C-C}$ & $3.0 \mathrm{E}-6 / \mathrm{h}$ & 10 \\
\hline Fails to respond & CMV-NR-C & $3.0 \mathrm{E}-6 / \mathrm{h}$ & 10 \\
\hline Plugs & CMV-PG-C & $5.0 \mathrm{E}-8 / \mathrm{h}$ & 10 \\
\hline Leakage (external) & CMV-LE-C & $5.0 \mathrm{E}-7 / \mathrm{h}$ & 10 \\
\hline Rupture (external) & CMV-RE-C & $3.0 \mathrm{E}-8 / \mathrm{h}$ & 30 \\
\hline $\begin{array}{l}\text { Air-Operated } \\
\text { Fails open }\end{array}$ & $\overline{C A V-F O-C}$ & $3.0 \mathrm{E}-6 / \mathrm{h}$ & 10 \\
\hline Fails closed & CAV-FC-C & $3.0 \mathrm{E}-6 / \mathrm{h}$ & 10 \\
\hline Fails to respond & CAV-NR-C & $\overline{3.0 \mathrm{E}}-\overline{6 / \mathrm{h}}$ & 10 \\
\hline Plugs & CAV-PG-C & $5.0 \mathrm{E}-8 / \mathrm{h}$ & 10 \\
\hline Leakage (external) & CAV-LE-C & $5.0 \mathrm{E}-7 / \mathrm{h}$ & 10 \\
\hline Rupture (extemal) & CAV-RE-C & $3.0 \mathrm{E} \cdot 8 / \mathrm{h}$ & 30 \\
\hline
\end{tabular}

- Note: Modification of the recommended mean for hourly failure rates may be appropriaie for components subjected to hostile radioactive or chemical environments. 


\begin{tabular}{|c|c|c|c|}
\hline \multirow[t]{2}{*}{ System/Component/Failure Mode } & \multirow[t]{2}{*}{ Identifier } & \multicolumn{2}{|c|}{$\begin{array}{l}\text { Recommended Failure Rate } \\
\text { Distribution (lognormal) }\end{array}$} \\
\hline & & Mean & Error Factor ${ }^{a}$ \\
\hline $\begin{array}{l}\text { Solenoid-Operated } \\
\text { Fails open }\end{array}$ & CSV-FO-C & $3.0 \mathrm{E}-6 / \mathrm{h}$ & 10 \\
\hline Fails closed & CSV-FC-C & $3.0 \mathrm{E}-6 / \mathrm{h}$ & 10 \\
\hline Fails to respond & CSV-NR-C & $3.0 \mathrm{E}-6 / \mathrm{h}$ & 10 \\
\hline Plugs & CSV-PG-C & $5.0 \mathrm{E}-8 / \mathrm{h}$ & 10 \\
\hline Leakage (external) & CSV-LE-C & $5.0 \mathrm{E}-7 / \mathrm{h}$ & 10 \\
\hline Rupture (external) & CSV-RE-C & $3.0 \mathrm{E}-8 / \mathrm{h}$ & 30 \\
\hline $\begin{array}{l}\text { Pump } \\
\text { Motor-Driven } \\
\text { Fails to start }\end{array}$ & MDP-FS-C & $1.0 \mathrm{E}-2 / \mathrm{d}$ & 10 \\
\hline Fails to run & MDP-FR-C & $1.0 \mathrm{E}-4 / \mathrm{h}$ & 10 \\
\hline Overspeed & MDP-OS-C & $3.0 \mathrm{E}-5 / \mathrm{h}$ & 10 \\
\hline Fails to stop & MDP-NS-C & $1.0 \mathrm{E}-2 / \mathrm{d}$ & 10 \\
\hline Leakage (external) & MDP-LE-C & $1.0 \mathrm{E}-6 / \mathrm{h}$ & 10 \\
\hline Rupture (external) & MDP-RE-C & $5.0 \mathrm{E}-8 / \mathrm{h}$ & 30 \\
\hline $\begin{array}{c}\text { Turbine-Driven } \\
\text { Fails to start }\end{array}$ & TDP-FS-C & $3.0 \mathrm{E}-2 / \mathrm{d}$ & 10 \\
\hline Fails to run & TDP-FR-C & $1.0 \mathrm{E}-4 / \mathrm{h}$ & 30 \\
\hline Overspeed & TDP-OS-C & $3.0 \mathrm{E}-5 / \mathrm{h}$ & 10 \\
\hline Fails to stop & TDP-NS-C & $3.0 \mathrm{E}-2 / \mathrm{d}$ & 10 \\
\hline Leakage (external) & TDP-LE-C & $1.0 \mathrm{E}-6 / \mathrm{h}$ & 10 \\
\hline Rupture (external) & TDP-RE-C & $5.0 \mathrm{E}-8 / \mathrm{h}$ & 30 \\
\hline $\begin{array}{l}\text { Diesel-Driven } \\
\text { Fails to start } \\
\end{array}$ & DDP-FS-C & $1.0 \mathrm{E}-2 / \mathrm{d}$ & 10 \\
\hline Fails to run & DDP-FR-C & $5.0 \mathrm{E}-3 / \mathrm{h}$ & 10 \\
\hline Overspeed & DDP-OS-C & $1.0 \mathrm{E}-3 / \mathrm{h}$ & 10 \\
\hline Fails to stop & DDP-NS-C & $1.0 \mathrm{~F} .-2 / \mathrm{d}$ & 10 \\
\hline Leakage (external) & DDP-LE-C & $1.0 \mathrm{E}-6 / \mathrm{h}$ & 10 \\
\hline Rupture (external) & DDP-RE-C & $5.0 \mathrm{E}-8 / \mathrm{h}$ & 30 \\
\hline $\begin{array}{l}\text { Piping/Hose/Jumper } \\
\text { Piping } \\
\text { Leakage (external) }\end{array}$ & PIP-LE-C & $3.0 \mathrm{E}-9 / \mathrm{h}-\mathrm{ft}$ & 10 \\
\hline Rupture (external) & PIP-RE-C & $1.0 \mathrm{E}-10 / \mathrm{h}-\mathrm{ft}$ & 30 \\
\hline Plugs & PIP-PG-C & $1.0 \mathrm{E}-10 / \mathrm{h}-\mathrm{ft}$ & 30 \\
\hline $\begin{array}{l}\text { Hose } \\
\text { Leakage (external) }\end{array}$ & HOS-LE-C & $1.0 \mathrm{E}-9 / \mathrm{h}-\mathrm{ft}$ & 10 \\
\hline Rupture (external) & HOS-RE-C & $1.0 \mathrm{E}-8 / \mathrm{h}-\mathrm{ft}$ & 10 \\
\hline Plugs & HOS-PG-C & $1.0 \mathrm{E}-8 / \mathrm{h}-\mathrm{ft}$ & 10 \\
\hline $\begin{array}{l}\text { Jumper } \\
\text { Leakage (external) }\end{array}$ & JPR-LE-C & $1.0 \mathrm{E}-6 / \mathrm{h}$ & 10 \\
\hline Rupture (external) & JPR-RE-C & $1.0 \mathrm{E}-8 / \mathrm{h}$ & 30 \\
\hline
\end{tabular}

Note: Modification of the recommended mean for hourly failure rates may be appropriate for components subjected to hostile radioactive or chemical environments. 


\begin{tabular}{|c|c|c|c|}
\hline \multirow[t]{2}{*}{ System/Component/Failure Mode } & \multirow[t]{2}{*}{ Identifier } & \multicolumn{2}{|c|}{$\begin{array}{l}\text { Recommended Failure Rate } \\
\text { Distribution (lognormal) }\end{array}$} \\
\hline & & Mean & Error Factor ${ }^{\mathrm{a}}$ \\
\hline Plugs & JPR-PG-C & $5.0 \mathrm{E}-8 / \mathrm{h}$ & 10 \\
\hline $\begin{array}{l}\text { Vessel } \\
\text { Tank (Unpressurized) } \\
\text { Leakage (external) }\end{array}$ & TKU-LE-C & $1.0 \mathrm{E}-7 / \mathrm{h}$ & 10 \\
\hline Rupture (external) & TKU-RE-C & $5.0 \mathrm{E}-9 / \mathrm{h}$ & 30 \\
\hline $\begin{array}{l}\text { Tank (Pressurized) } \\
\text { Leakage (external) }\end{array}$ & TKP-LE-C & $1.0 \mathrm{E}-7 / \mathrm{h}$ & 10 \\
\hline Rupture (external) & TKP-RE-C & $5.0 \mathrm{E}-9 / \mathrm{h}$ & 30 \\
\hline $\begin{array}{l}\text { Flange/Gasket } \\
\text { Leakage (external) }\end{array}$ & FLG-LE-C & $1.0 \mathrm{E}-7 / \mathrm{h}$ & 10 \\
\hline Rupture (external) & FLG-RE-C & $1.0 \mathrm{E}-9 / \mathrm{h}$ & 10 \\
\hline $\begin{array}{l}\text { Heat Exchanger } \\
\text { Shell/Tube } \\
\text { Fouling (tubes) }\end{array}$ & HTX-FL-C & $1.0 \mathrm{E}-6 / \mathrm{h}$ & 10 \\
\hline Plugs (rubes) & HTX-PG-C & $3.0 \mathrm{E}-7 / \mathrm{h}$ & 10 \\
\hline Leakage (rubes) & HTX-LI-C & $1.0 \mathrm{E}-6 / \mathrm{h}$ & 10 \\
\hline Rupturc (tubes) & HTX-RI-C & $5.0 \mathrm{E}-8 / \mathrm{h}$ & 30 \\
\hline Leakage (shell) & HTX-LE-C & $1.0 \mathrm{E}-7 / \mathrm{h}$ & 10 \\
\hline Rupture (shell) & HTX-RE-C & $5.0 \mathrm{E}-9 / \mathrm{h}$ & 30 \\
\hline $\begin{array}{l}\text { Heater (Electrical) } \\
\text { Fails to heat }\end{array}$ & HTE-FH-C & $1.0 \mathrm{E}-5 / \mathrm{h}$ & 10 \\
\hline Overheats & HTE-OH-C & $3.0 \mathrm{E}-6 / \mathrm{h}$ & 10 \\
\hline Leakage (external) & HTE-LE-C & $1.0 \mathrm{E}-6 / \mathrm{h}$ & 10 \\
\hline Rupture (external) & HTE-RE-C & $5.0 \mathrm{E}-8 / \mathrm{h}$ & 30 \\
\hline $\begin{array}{l}\text { Strainer/Filter } \\
\text { Plugs }\end{array}$ & FLT-PG-C & $3.0 \mathrm{E}-6 / \mathrm{h}$ & 10 \\
\hline Leakage (internal) & FLT-LI-C & $3.0 \mathrm{E}-6 / \mathrm{h}$ & 10 \\
\hline Rupture (internal) & FLT-RI-C & $5.0 \mathrm{E}-7 / \mathrm{h}$ & 10 \\
\hline $\begin{array}{l}\text { Orifice } \\
\text { Plugs }\end{array}$ & ORF-PG-C & $1.0 \mathrm{E} \cdot 6 / \mathrm{h}$ & 10 \\
\hline $\begin{array}{l}\text { Miscellaneous } \\
\text { Mixer/Blender } \\
\text { Failure }\end{array}$ & MIX-FA-C & $5.0 \mathrm{E}-6 / \mathrm{h}$ & 10 \\
\hline $\begin{array}{l}\text { Agitator } \\
\text { Failure }\end{array}$ & AGI-FA-C & $5.0 \mathrm{E}-6 / \mathrm{h}$ & 10 \\
\hline $\begin{array}{l}\text { Centrifuge } \\
\text { Failure }\end{array}$ & CTF-FA-C & $5.0 \mathrm{E}-6 / \mathrm{h}$ & 10 \\
\hline
\end{tabular}

Table 1b. Chemical process system recommended generic failure rates.

a. Error factor is the 95 th percentile/50th percentile.

- Notc: Modification of the recommended mean for hourly failure rates may be appropriate for components subjected to hostile radioactive or chemical environments. 


\begin{tabular}{|c|c|c|c|}
\hline \multirow[t]{2}{*}{ System/Component/Failure Mode } & \multirow[t]{2}{*}{ Identifier } & \multicolumn{2}{|c|}{$\begin{array}{l}\text { Recommended Failure Rate } \\
\text { Distribution (lognormal) }\end{array}$} \\
\hline & & Mean & Error Factor ${ }^{a}$ \\
\hline $\begin{array}{l}\text { Compressed Gas } \\
\text { Valve (Standby or Safety) } \\
\text { Manual } \\
\text { Fails to open/close }\end{array}$ & $\begin{array}{l}\text { XVM-CC-G } \\
\text { or } \\
\text { XVM-OO-G }\end{array}$ & $1.0 \mathrm{E}-3 / \mathrm{d}$ & 10 \\
\hline Plugs & XVM-PG-G & $5.0 \mathrm{E}-7 / \mathrm{h}$ & 10 \\
\hline Leakage (internal) & XVM-LI-G & $1.0 \mathrm{E}-5 / \mathrm{h}$ & 10 \\
\hline Rupture (intemal) & XVM-RI-G & $5.0 \mathrm{E}-7 / \mathrm{h}$ & 30 \\
\hline Leakage (external) & XVM-LE-G & $1.0 \mathrm{E}-7 / \mathrm{h}$ & 10 \\
\hline Rupture (external) & XVM-RE-G & $5.0 \mathrm{E}-9 / \mathrm{h}$ & 30 \\
\hline $\begin{array}{l}\text { Check } \\
\text { Fails to open }\end{array}$ & CKV-CC-G & $1.0 \mathrm{E}-4 / \mathrm{d}$ & 10 \\
\hline Fails to close & CKV-OOG & $3.0 \mathrm{E}-3 / \mathrm{d}$ & 10 \\
\hline Plugs & CKV-PG-G & $5.0 \mathrm{E}-7 / \mathrm{h}$ & 10 \\
\hline Leakage (intemal) & CKV-LI-G & $1.0 \mathrm{E}-5 / \mathrm{h}$ & 10 \\
\hline Rupture (intemal) & CKV-RI-G & $5.0 \mathrm{E}-7 / \mathrm{h}$ & 30 \\
\hline Leakage (external) & CKV-LE-G & $1.0 \mathrm{E}-7 / \mathrm{h}$ & 10 \\
\hline Rupture (external) & CKV-RE-G & $5.0 \mathrm{E}-9 / \mathrm{h}$ & 30 \\
\hline $\begin{array}{l}\text { Motor-Operated } \\
\text { Fails to open/close }\end{array}$ & $\begin{array}{l}\text { MOV-CC-G } \\
\text { or } \\
\text { MOV-OO-G }\end{array}$ & $1.0 \mathrm{E}-2 / \mathrm{d}$ & 10 \\
\hline Spurious operation & $\begin{array}{l}\text { MOV-CO-G } \\
\text { or } \\
\text { MOV-OC-G }\end{array}$ & $3.0 \mathrm{E}-7 / \mathrm{h}$ & 10 \\
\hline Plugs & MOV-PG-G & $5.0 \mathrm{E}-7 / \mathrm{h}$ & 10 \\
\hline Leakage (internal) & MOV-LI-G & $1.0 \mathrm{E}-5 / \mathrm{h}$ & 10 \\
\hline Rupture (internal) & MOV-RI-G & $5.0 \mathrm{E}-7 / \mathrm{h}$ & 30 \\
\hline Leakage (external) & MOV-LE-G & $1.0 \mathrm{E}-7 / \mathrm{h}$ & 10 \\
\hline Rupture (external) & MOV-RE-G & $5.0 \mathrm{E}-9 / \mathrm{h}$ & 30 \\
\hline $\begin{array}{l}\text { Air-Operated } \\
\text { Fails to open/close }\end{array}$ & $\begin{array}{l}\text { AOV-CC-G } \\
\text { or } \\
\text { AOV-OO-G }\end{array}$ & $3.0 \mathrm{E}-3 / \mathrm{d}$ & 30 \\
\hline Spurious operation & $\begin{array}{l}\text { AOV-COG } \\
\text { or } \\
\text { AOV-OC-G }\end{array}$ & $1.0 \mathrm{E}-6 / \mathrm{h}$ & 10 \\
\hline Plugs & AOV-PG-G & $5.0 \mathrm{E}-7 / \mathrm{h}$ & 10 \\
\hline Leakage (internal) & AOV-LI-G & $1.0 \mathrm{E}-5 / \mathrm{h}$ & 10 \\
\hline Rupture (internal) & AOV-RI-G & $5.0 \mathrm{E}-7 / \mathrm{h}$ & 30 \\
\hline Leakage (external) & AOV-LE-G & $1.0 \mathrm{E}-7 / \mathrm{h}$ & 10 \\
\hline Rupture (external) & AOV-RE-G & $5.0 \mathrm{E}-9 / \mathrm{h}$ & 30 \\
\hline $\begin{array}{l}\text { Solenoid-Operated } \\
\text { Fails to open/close }\end{array}$ & $\begin{array}{l}\text { SOV-CC-G } \\
\text { or } \\
\text { SOV-OOG }\end{array}$ & $3.0 \mathrm{E}-3 / \mathrm{d}$ & 10 \\
\hline Spurious operation & $\begin{array}{l}\text { SOV-CO-G } \\
\text { or } \\
\text { SOV-OC-G } \\
\end{array}$ & $5.0 \mathrm{E}-7 / \mathrm{h}$ & 10 \\
\hline
\end{tabular}

Note: Modification of the recommended mean for hourly failure rates may be appropriate for components subjected to hostile radioactive or chemical environments. 
WSRC.TR-93-262

\begin{tabular}{|c|c|c|c|}
\hline \multirow[t]{2}{*}{ System/Component/Failure Mode } & \multirow[t]{2}{*}{ Identifier } & \multicolumn{2}{|c|}{$\begin{array}{l}\text { Recommended Failure Rate } \\
\text { Distribution (lognormal) }\end{array}$} \\
\hline & & Mean & Error Faclor ${ }^{\mathrm{a}}$ \\
\hline Plugs & SOV-PG-G & $\overline{5.0 \mathrm{E}-7 / \mathrm{h}}$ & 10 \\
\hline Leakage (internal) & SOV-LI-G & $1.0 \mathrm{E}-5 / \mathrm{h}$ & 10 \\
\hline Rupture (intemal) & SOV-RI-G & $5.0 \mathrm{E}-7 / \mathrm{h}$ & 30 \\
\hline Leakage (external) & SOV-LE-G & $1.0 \mathrm{E}-7 / \mathrm{h}$ & 10 \\
\hline Rupture (external) & SOV-RE-G & $5.0 \mathrm{E}-9 / \mathrm{h}$ & 30 \\
\hline $\begin{array}{l}\text { Safety/Relief } \\
\text { Fails to open } \\
\end{array}$ & SRV-CC-G & $1.0 \mathrm{E}-2 / \mathrm{d}$ & 10 \\
\hline Fails to reclose & SRV-OO-G & $1.0 \mathrm{E}-2 / \mathrm{d}$ & 10 \\
\hline Leakage (internal) & SRV-LI-G & $1.0 \mathrm{E}-5 / \mathrm{h}$ & 10 \\
\hline Rupture (internal) & SRV-RI-G & $5.0 \mathrm{E}-7 / \mathrm{h}$ & $\overline{30}$ \\
\hline Leakage (external) & SRV-LE-G & $1.0 \mathrm{E}-7 / \mathrm{h}$ & 10 \\
\hline Rupture (external) & SRV-RE-G & $5.0 \mathrm{E}-9 / \mathrm{h}$ & 30 \\
\hline $\begin{array}{l}\text { Vacuum-Breaker } \\
\text { Fails to open }\end{array}$ & VBV-CC-G & $3.0 \mathrm{E}-2 / \mathrm{d}$ & 10 \\
\hline Fails to reclose & VBV-OO-G & $3.0 \mathrm{E}-2 / \mathrm{d}$ & 10 \\
\hline Leakage (intcmal) & VBV-LI-G & $1.0 \mathrm{E} .5 / \mathrm{h}$ & 10 \\
\hline Rupture (internal) & VBV-RI-G & $5.0 \mathrm{E}-7 / \mathrm{h}$ & 30 \\
\hline Leakage (external) & VBV-LE-G & $1.0 \mathrm{E}-7 / \mathrm{h}$ & 10 \\
\hline Rupture (external) & VBV-RE-G & $5.0 \mathrm{E}-9 / \mathrm{h}$ & 30 \\
\hline $\begin{array}{l}\text { Valve (Control) } \\
\text { Motor-Operated } \\
\text { Fails open }\end{array}$ & $\overline{C M V-F O-G}$ & $3.0 \mathrm{E}-6 / \mathrm{h}$ & 10 \\
\hline Fails closed & CMV-FC-G & $3.0 \mathrm{E}-6 / \mathrm{h}$ & 10 \\
\hline Fails to respond & CMV-NR-G & $3.0 \mathrm{E}-6 / \mathrm{h}$ & 10 \\
\hline Plugs & CMV-PG-G & $5.0 \mathrm{E}-7 / \mathrm{h}$ & 10 \\
\hline Leakage (external) & CMV-LE-G & $1.0 \mathrm{E}-7 / \mathrm{h}$ & 10 \\
\hline Rupture (external) & CMV-RE-G & $5.0 \mathrm{E}-9 / \mathrm{h}$ & 30 \\
\hline $\begin{array}{l}\text { Air-Operated } \\
\text { Fails open }\end{array}$ & CAV-FO-G & $3.0 \mathrm{E}-6 / \mathrm{h}$ & 10 \\
\hline Fails closed & CAV-FC-G & $3.0 \mathrm{E}-6 / \mathrm{h}$ & 10 \\
\hline Fails to respond & CAV-NR-G & $3.0 \mathrm{E}-6 / \mathrm{h}$ & 10 \\
\hline Plugs & CAV.PG-G & $5.0 \mathrm{E}-7 / \mathrm{h}$ & 10 \\
\hline Leakage (external) & CAV-LE-G & $1.0 \mathrm{E}-7 / \mathrm{h}$ & 10 \\
\hline Rupture (external) & CAV-RE-G & $5.0 \mathrm{E}-9 / \mathrm{h}$ & 30 \\
\hline $\begin{array}{l}\text { Solenoid-Operated } \\
\text { Fails open }\end{array}$ & CSV-FO-G & $3.0 \mathrm{E}-6 / \mathrm{h}$ & 10 \\
\hline Fails closed & CSV-FC-G & $3.0 \mathrm{E}-6 / \mathrm{h}$ & 10 \\
\hline Fails to respond & CSV-NR-G & $3.0 \mathrm{E}-6 / \mathrm{h}$ & 10 \\
\hline Plugs & CSV-PG-G & $5.0 \mathrm{E}-7 / \mathrm{h}$ & 10 \\
\hline laid (evtcrnal) & CSV-LE-G & $1.05 .7 n$ & 10 \\
\hline
\end{tabular}

- No Modification of the recommended mean for hourly failure rates may be appropriate for components subjected to hostile radioactive or chemical environments. 


\begin{tabular}{|c|c|c|c|}
\hline \multirow[t]{2}{*}{ System/Component/Failure Mode } & \multirow[t]{2}{*}{ Identifier } & \multicolumn{2}{|c|}{$\begin{array}{l}\text { Recommended Failure Rate } \\
\text { Distribution (lognormal) }\end{array}$} \\
\hline & & Mean & Error Factor ${ }^{a}$ \\
\hline Rupture (external) & CSV-RE-G & $5.0 \mathrm{E}-9 / \mathrm{h}$ & 30 \\
\hline $\begin{array}{l}\text { Compressor } \\
\text { Motor-Driven } \\
\text { Fails to start } \\
\end{array}$ & MDC-FS-G & $5.0 \mathrm{E}-3 / \mathrm{d}$ & $\overline{5}$ \\
\hline Fails to run & $\overline{M D C-F R-G}$ & $5.0 \mathrm{E}-5 / \mathrm{h}$ & 3 \\
\hline Overspeed & MDC-OS-G & $1.0 \mathrm{E}-5 / \mathrm{h}$ & 5 \\
\hline Fails to stop & MDC-NS-G & $5.0 \mathrm{E}-3 / \mathrm{d}$ & 5 \\
\hline Leakage (external) & MDC-LE-G & $3.0 \mathrm{E}-7 / \mathrm{h}$ & 10 \\
\hline Rupture (external) & MDC-RE-G & $1.0 \mathrm{E}-8 / \mathrm{h}$ & 30 \\
\hline $\begin{array}{l}\text { Piping/Hose/Jumper/Tube } \\
\text { Piping } \\
\text { Leakage (external) }\end{array}$ & PIP-LE-G & $3.0 \mathrm{E}-8 / \mathrm{h}-\mathrm{ft}$ & 10 \\
\hline Rupture (external) & PIP-RE-G & $1.0 \mathrm{E}-9 / \mathrm{h}-\mathrm{ft}$ & 30 \\
\hline Plugs & PIP-PG-G & $1.0 \mathrm{E}-9 / \mathrm{h}-\mathrm{ft}$ & 30 \\
\hline $\begin{array}{l}\text { Hose } \\
\text { Leakage (external) }\end{array}$ & HOS-LE-G & $1.0 \mathrm{E}-8 / \mathrm{h}-\mathrm{ft}$ & 10 \\
\hline Rupture (external) & HOS-RE-G & $1.0 \mathrm{E}-7 / \mathrm{h}-\mathrm{ft}$ & 10 \\
\hline Plugs & HOS-PG-G & $1.0 \mathrm{E}-7 / \mathrm{h}-\mathrm{ft}$ & 10 \\
\hline $\begin{array}{l}\text { Jumper } \\
\text { Leakage (external) }\end{array}$ & JPR-LE-G & $1.0 \mathrm{E}-5 / \mathrm{h}$ & 10 \\
\hline Rupture (external) & JPR-RE-G & $1.0 \mathrm{E}-7 / \mathrm{h}$ & 30 \\
\hline Plugs & JPR-PG-G & $1.0 \mathrm{E}-7 / \mathrm{h}$ & 30 \\
\hline $\begin{array}{l}\text { Tube } \\
\text { Leakage (external) }\end{array}$ & TUB-LE-G & $3.0 \mathrm{E}-7 / \mathrm{h}-\mathrm{ft}$ & 10 \\
\hline Rupture (external) & TUB-RE-G & $1.0 \mathrm{E}-8 / \mathrm{h}-\mathrm{ft}$ & 30 \\
\hline Plugs & TUB-PG-G & $1.0 \mathrm{E}-8 / \mathrm{h}-\mathrm{ft}$ & 30 \\
\hline $\begin{array}{l}\text { Vessel } \\
\text { Tank (Pressurized) } \\
\text { Leakage (external) }\end{array}$ & TKP-LE-G & $1.0 \mathrm{E}-7 / \mathrm{h}$ & 10 \\
\hline Rupture (external) & TKP-RE-G & $5.0 \mathrm{E}-9 / \mathrm{h}$ & 30 \\
\hline $\begin{array}{l}\text { Cylinder (Pressurized) } \\
\text { Leakage (external) }\end{array}$ & CYL-LE-G & $1.0 \mathrm{E}-7 / \mathrm{h}$ & 10 \\
\hline Rupture (external) & CYL-RE-G & $5.0 \mathrm{E}-9 / \mathrm{h}$ & 30 \\
\hline $\begin{array}{l}\text { Flange/Gasket } \\
\text { Leakage (external) }\end{array}$ & FLG-LE-G & $1.0 \mathrm{E}-7 / \mathrm{h}$ & 10 \\
\hline Rup ure (external) & FLG-RE-G & $1.0 \mathrm{E}-9 / \mathrm{h}$ & 10 \\
\hline $\begin{array}{l}\text { Heat Exchanger } \\
\text { Shell/Tube } \\
\text { Fouling (tubes) }\end{array}$ & HTX-FL-G & $1.0 \mathrm{E}-5 / \mathrm{h}$ & 10 \\
\hline Plugs (tubes) & HTX-PG-G & $3.0 \mathrm{E}-6 / \mathrm{h}$ & 10 \\
\hline Leakage (tubes) & HTX-LI-G & $1.0 \mathrm{E}-5 / \mathrm{h}$ & 10 \\
\hline Rupture (tubes) & HTX-RI-G & $5.0 \mathrm{E}-7 / \mathrm{h}$ & 30 \\
\hline Leakage (shell) & HTX-LE-G & $1.0 \mathrm{E}-6 / \mathrm{h}$ & 10 \\
\hline Rupture (shell) & HTX-RE-G & $5.0 \mathrm{E}-8 / \mathrm{h}$ & 30 \\
\hline
\end{tabular}

Note: Modification of the recommended mean for hourly failure rates may be appropriate for components subjected to hostile radioactive or chemical environments. 
WSRC-TR-93-262

\begin{tabular}{||c|l|l|l||}
\hline Table 1c. Compressed gas system recommended gencric failure rates (continued). \\
\hline System/Component/Failure Mode & \multicolumn{1}{|c|}{ Identificr } & \multicolumn{2}{|c|}{$\begin{array}{c}\text { Recommended Failure Rate } \\
\text { Distribution (lognormal) }\end{array}$} \\
\cline { 2 - 4 } & & \multicolumn{1}{|c|}{ Mean } & \multicolumn{1}{|c|}{ Error Factor ${ }^{\mathrm{a}}$} \\
\hline $\begin{array}{c}\text { Heater (Electrical) } \\
\text { Fails to heat }\end{array}$ & HTE-FH-G & $1.0 \mathrm{E}-6 / \mathrm{h}$ & 10 \\
\hline Overheats & HTE-OH-G & $3.0 \mathrm{E}-7 / \mathrm{h}$ & 10 \\
\hline Leakage (external) & HTE-LE-G & $1.0 \mathrm{E}-6 / \mathrm{h}$ & 10 \\
\hline Rupture (external) & HTE-RE-G & $5.0 \mathrm{E}-8 / \mathrm{h}$ & 30 \\
\hline $\begin{array}{c}\text { Vaporizer } \\
\text { Failure }\end{array}$ & VAP-FA-G & $1.0 \mathrm{E}-4 / \mathrm{h}$ & 10 \\
\hline $\begin{array}{c}\text { Air Dryer } \\
\text { Failure }\end{array}$ & ADR-FA-G & $5.0 \mathrm{E}-6 / \mathrm{h}$ & 10 \\
\hline $\begin{array}{c}\text { Filter } \\
\text { Plugs }\end{array}$ & FLT-PG-G & $3.0 \mathrm{E}-6 / \mathrm{h}$ & 10 \\
\hline Leakage (internal) & FLT-LI-G & $3.0 \mathrm{E}-6 / \mathrm{h}$ & 10 \\
\hline Rupture (internal) & FLT-RI-G & $5.0 \mathrm{E}-7 / \mathrm{h}$ & 10 \\
\hline $\begin{array}{c}\text { Orifice } \\
\text { Plugs }\end{array}$ & ORF-PG-G & $1.0 \mathrm{E}-6 / \mathrm{h}$ & 10 \\
\hline
\end{tabular}

a. Error factor is the 95th percentile/50th percentile.

Note: Modification of the recommended mean for hourly failure rates may be appropriate for components subjected to hostile radioactive or chemical environments. 


\begin{tabular}{|c|c|c|c|}
\hline \multirow[t]{2}{*}{ System/Component/Failure Mode } & \multirow[t]{2}{*}{ Identifier } & \multicolumn{2}{|c|}{$\begin{array}{l}\text { Recommended Failure Rate } \\
\text { Distribution (lognormal) }\end{array}$} \\
\hline & & Mean & Error Factor ${ }^{a}$ \\
\hline $\begin{array}{l}\text { HVAC/Exhaust } \\
\text { Damper (Standby or Safety) } \\
\text { Manual } \\
\text { Fails to open/close }\end{array}$ & $\begin{array}{l}\text { XDM-CC-H or } \\
\text { XDM-OO-H }\end{array}$ & $3.0 \mathrm{E}-3 / \mathrm{d}$ & 10 \\
\hline Plugs & XDM-PG-H & $5.0 \mathrm{E}-7 / \mathrm{h}$ & 10 \\
\hline Leakage (intemal) & XDM-LI-H & $1.0 \mathrm{E}-5 / \mathrm{h}$ & 10 \\
\hline Rupture (intemal) & XDM-RI-H & $5.0 \mathrm{E}-7 / \mathrm{h}$ & 30 \\
\hline Leakage (external) & XDM-LE-H & $1.0 \mathrm{E}-7 / \mathrm{h}$ & 10 \\
\hline Rupture (external) & XDM-RE-H & $5.0 \mathrm{E}-9 / \mathrm{h}$ & 30 \\
\hline $\begin{array}{l}\text { Motor-Operated } \\
\text { Fails to open/close }\end{array}$ & $\begin{array}{l}\text { MOD-CC-H or } \\
\text { MODOO-H }\end{array}$ & $3.0 \mathrm{E}-2 / \mathrm{d}$ & 10 \\
\hline Spurious operation & $\begin{array}{l}\text { MOD-CO-H or } \\
\text { MOD-OC-H }\end{array}$ & $3.0 \mathrm{E}-6 / \mathrm{h}$ & 10 \\
\hline Plugs & MOD-PG-H & $5.0 \mathrm{E}-7 / \mathrm{h}$ & 10 \\
\hline Leakage (intemal) & MOD-L-H & $1.0 \mathrm{E}-5 / \mathrm{h}$ & 10 \\
\hline Rupture (internal) & $\overline{M O D-R I-H}$ & $5.0 \mathrm{E}-7 / \mathrm{h}$ & 10 \\
\hline Leakage (external) & MOD-LE-H & $1.0 \mathrm{E} \cdot 7 / \mathrm{h}$ & 10 \\
\hline Rupture (external) & MOD-RE-H & $5.0 \mathrm{E}-9 / \mathrm{h}$ & 30 \\
\hline $\begin{array}{l}\text { Air-Operated } \\
\text { Fails to open/close }\end{array}$ & $\begin{array}{l}\text { AOD-CC-H or } \\
\text { AOD-OO-H }\end{array}$ & $1.0 \mathrm{E}-2 / \mathrm{d}$ & 30 \\
\hline Spurious operation & $\begin{array}{l}\text { AOD-CO-H or } \\
\text { AOD-OC.H }\end{array}$ & $1.0 \mathrm{E}-5 / \mathrm{h}$ & 10 \\
\hline Plugs & AOD-PG-H & $5.0 \mathrm{E}-7 / \mathrm{h}$ & 10 \\
\hline Leakage (intemal) & AOD-LI-H & $1.0 \mathrm{E}-5 / \mathrm{h}$ & 10 \\
\hline Rupture (internal) & AOD-RI-H & $5.0 \mathrm{E}-7 / \mathrm{h}$ & 10 \\
\hline Leakage (external) & $\overline{\text { AOD-LE-H }}$ & $1.0 \mathrm{E}-7 / \mathrm{h}$ & 10 \\
\hline Rupture (external) & AOD-RE-H & $5.0 \mathrm{E}-9 / \mathrm{h}$ & 30 \\
\hline $\begin{array}{l}\text { Damper (Control) } \\
\text { Motor-Operated } \\
\text { Fails open }\end{array}$ & CMD-FO-H & $3.0 \mathrm{E}-6 / \mathrm{h}$ & 10 \\
\hline Fails closed & CMD-FC-H & $3.0 \mathrm{E}-6 / \mathrm{h}$ & 10 \\
\hline Fails to respond & CMD-NR-H & $3.0 \mathrm{E}-6 / \mathrm{h}$ & 10 \\
\hline Plugs & CMD-PG-H & $5.0 \mathrm{E}-7 / \mathrm{h}$ & 10 \\
\hline Leakage (external) & CMD-LE-H & $1.0 \mathrm{E}-7 / \mathrm{h}$ & 10 \\
\hline Rupture (external) & CMD-RE-H & $5.0 \mathrm{E}-9 / \mathrm{h}$ & 30 \\
\hline $\begin{array}{l}\text { Air-Operated } \\
\text { Fails open }\end{array}$ & CAD-FO-H & $3.0 \mathrm{E}-6 / \mathrm{h}$ & 10 \\
\hline Fails closed & CAD-FC-H & $3.0 \mathrm{E}-6 / \mathrm{h}$ & 10 \\
\hline Fails to respond & CAD-NR-H & $3.0 \mathrm{E}-6 / \mathrm{h}$ & 10 \\
\hline Plugs & CAD-PG-H & $5.0 \mathrm{E}-7 / \mathrm{h}$ & 10 \\
\hline Leakage (external) & CAD-LE-H & $1.0 \mathrm{E}-7 / \mathrm{h}$ & 10 \\
\hline Rupture (external) & CAD-RE-H & $5.0 \mathrm{E}-9 / \mathrm{h}$ & 30 \\
\hline
\end{tabular}

Note: Modification of the recommended mean for hourly failure rates may be appropriate for components subjected to hostile radioactive or chemical environments. 


\begin{tabular}{|c|c|c|c|}
\hline \multirow[t]{2}{*}{ System/Component/Failure Mode } & \multirow[t]{2}{*}{ Identifier } & \multicolumn{2}{|c|}{$\begin{array}{l}\text { Recommended Failure Rate } \\
\text { Distribution (lognormal) }\end{array}$} \\
\hline & & Mean & Error Factor \\
\hline $\begin{array}{l}\text { Fan } / \text { Blower } \\
\text { Motor-Driven } \\
\text { Fails to start }\end{array}$ & MDF-FS-H & $5.0 \mathrm{E}-3 / \mathrm{d}$ & 5 \\
\hline Fails to nun & MDF-FR-H & $3.0 \mathrm{E}-5 / \mathrm{h}$ & 3 \\
\hline Overspeed & MDF-OS-H & $5.0 \mathrm{E}-6 / \mathrm{h}$ & 10 \\
\hline Fails to stop & MDF-NS-H & $5.0 \mathrm{E}-3 / \mathrm{d}$ & 10 \\
\hline Leakage (external) & MDF-LE-H & $3.0 \mathrm{E}-7 / \mathrm{h}$ & 10 \\
\hline Rupture (external) & MDF-RE-H & $1.0 \mathrm{E}-8 / \mathrm{h}$ & 30 \\
\hline $\begin{array}{l}\text { Diesel-Driven } \\
\text { Fails to start }\end{array}$ & $\overline{D D F} \cdot \mathrm{FS} \cdot \mathrm{H}$ & $1.0 \mathrm{E}-2 / \mathrm{d}$ & 10 \\
\hline Fails to run & DDF-FR-H & $5.0 \mathrm{E} \cdot 3 / \mathrm{h}$ & 10 \\
\hline Overspeed & DDF-OS-H & $\overline{1.0 \mathrm{E}-3 / \mathrm{h}}$ & 10 \\
\hline Fails to stop & $\mathrm{DDF} \cdot \mathrm{NS} \cdot \mathrm{H}$ & $1.0 \mathrm{E}-2 / \mathrm{d}$ & 10 \\
\hline Leakage (extemal) & DDF-LE-H & $3.0 \mathrm{E}-7 / \mathrm{h}$ & 10 \\
\hline Rupture (external) & DDF-RE-H & $1.0 \mathrm{E}-8 / \mathrm{h}$ & 10 \\
\hline $\begin{array}{l}\text { Ducting } \\
\text { Leakage (external) }\end{array}$ & DCT-LE-H & $3.0 \mathrm{E}-7 / \mathrm{h}-\mathrm{ft}$ & 10 \\
\hline Rupture (external) & DCT-RE-H & $1.0 \mathrm{E} \cdot 8 / \mathrm{h}-\mathrm{ft}$ & 30 \\
\hline Plugs & DCT.PG-H & $1.0 \mathrm{E}-8 / \mathrm{h}-\mathrm{ft}$ & 30 \\
\hline $\begin{array}{l}\text { Heat Exchanger } \\
\text { Air Conditioning Unit/ } \\
\text { Chiller } \\
\text { Fails to start } \\
\end{array}$ & ACU-FS.H & $1.0 \mathrm{E}-2 / \mathrm{d}$ & 10 \\
\hline Fails to run & ACU-FR-H & $3.0 \mathrm{E}-5 / \mathrm{h}$ & 10 \\
\hline $\begin{array}{c}\text { Fan Cooler Unit } \\
\text { Fails to start }\end{array}$ & FCU-FS-H & $1.0 \mathrm{E}-2 / \mathrm{d}$ & 5 \\
\hline Fails to nun & FCU-FR-H & $1.0 \mathrm{E}-5 / \mathrm{h}$ & 3 \\
\hline $\begin{array}{l}\text { Heater (Electrical) } \\
\text { Fails to heat }\end{array}$ & HTE-FH-H & $1.0 \mathrm{E}-6 / \mathrm{h}$ & 3 \\
\hline Overheats & HTE-OH-H & $3.0 \mathrm{E}-7 / \mathrm{h}$ & 10 \\
\hline $\begin{array}{l}\text { Heater (Gas) } \\
\text { Fails to heat }\end{array}$ & HTG-FH-H & $1.0 \mathrm{E}-3 / \mathrm{h}$ & 10 \\
\hline Overheats & HTG-OH-H & $3.0 \mathrm{E} \cdot 4 / \mathrm{h}$ & 10 \\
\hline $\begin{array}{l}\text { Filter } \\
\text { Normal } \\
\text { Plugs } \\
\end{array}$ & FLT-PG-H & $3.0 \mathrm{E}-6 / \mathrm{h}$ & 10 \\
\hline Leakage (intemal) & FLT-LI-H & $3.0 \mathrm{E}-6 / \mathrm{h}$ & 10 \\
\hline Rupture (internal) & FLT-RI-H & $5.0 \mathrm{E}-7 / \mathrm{h}$ & 10 \\
\hline $\begin{array}{l}\text { Low-Efficiency } \\
\text { Plugs }\end{array}$ & FLLPG-H & $3.0 \mathrm{E}-6 / \mathrm{h}$ & 10 \\
\hline Lcakage (intemal) & FLLLLI-H & $3.0 \mathrm{E}-6 / \mathrm{h}$ & 10 \\
\hline Rupture (internal) & FLL-RI-H & $5.0 \mathrm{E}-7 / \mathrm{h}$ & 10 \\
\hline $\begin{array}{l}\text { HEPA } \\
\text { Plugs } \\
\end{array}$ & HPA-PG-H & $3.0 \mathrm{E}-6 / \mathrm{h}$ & 10 \\
\hline Leakage (internal) & HPA-LI-H & $3.0 \mathrm{E}-6 / \mathrm{h}$ & 10 \\
\hline Rupture (internal) & HPA-RI-H & $5.0 \mathrm{E}-7 / \mathrm{h}$ & 10 \\
\hline
\end{tabular}

Note: Modification of the recommended mean for hourly failure rates may be appropriate for components subjected to hostile radioactive or chemical environments. 


\begin{tabular}{|c|c|c|c|}
\hline \multirow[t]{2}{*}{ System/Component/Failurc Mode } & \multirow[t]{2}{*}{ Identifier } & \multicolumn{2}{|c|}{$\begin{array}{l}\text { Recommended Failure Rate } \\
\text { Distribution (lognormal) }\end{array}$} \\
\hline & & Mean & Error Factor ${ }^{a}$ \\
\hline $\begin{array}{l}\text { Sand } \\
\text { Plugs }\end{array}$ & FLS-PG-H & $3.0 \mathrm{E}-6 / \mathrm{h}$ & 10 \\
\hline Leakage (intemal) & FLS-LI-H & $3.0 \mathrm{E}-6 / \mathrm{h}$ & 10 \\
\hline Rupture (intemal) & FLS-RI-H & $5.0 \mathrm{E}-7 / \mathrm{h}$ & 10 \\
\hline $\begin{array}{l}\text { Baghouse } \\
\text { Plugs } \\
\end{array}$ & BAG-PG-H & $3.0 \mathrm{E}-5 / \mathrm{h}$ & 10 \\
\hline Leakage (intemal) & BAG-LI-H & $3.0 \mathrm{E}-5 / \mathrm{h}$ & 10 \\
\hline Rupture (internal) & BAG-RI-H & $5.0 \mathrm{E}-6 / \mathrm{h}$ & 10 \\
\hline $\begin{array}{l}\text { Miscellaneous } \\
\text { Mist Eliminator } \\
\text { Failure } \\
\end{array}$ & MTE-FA-H & $1.0 \mathrm{E}-4 / \mathrm{h}$ & 10 \\
\hline $\begin{array}{l}\text { Scrubber } \\
\text { Failure }\end{array}$ & SBR-FA-H & $1.0 \mathrm{E}-6 / \mathrm{h}$ & 10 \\
\hline $\begin{array}{l}\text { Precipitator } \\
\text { Failure } \\
\end{array}$ & PCP-FA-H & $5.0 \mathrm{E} \cdot 5 / \mathrm{h}$ & 10 \\
\hline
\end{tabular}

a. Error factor is the 95 th percentile/50th percentile.

Note: Modification of the recommended mean for hourly failure rates may be appropriate for components subjected to hostile radioactive or chemical environments. 


\begin{tabular}{|c|c|c|c|}
\hline \multirow[t]{2}{*}{ System/Componen//Failure Mode } & \multirow[t]{2}{*}{ Identifier } & \multicolumn{2}{|c|}{$\begin{array}{l}\text { Recommended Failure Rate } \\
\text { Distribution (lognormal) }\end{array}$} \\
\hline & & Mean & Error Factor \\
\hline $\begin{array}{l}\text { Electric Power } \\
\text { Generator } \\
\text { Diesel-Driven } \\
\text { Fails to start }\end{array}$ & DDG.FS-E & $1.0 \mathrm{E}-2 / \mathrm{d}$ & 3 \\
\hline Fails to nun & DDG-FR-E & $5.0 \mathrm{E}-3 / \mathrm{h}$ & 3 \\
\hline $\begin{array}{l}\text { Motor-Driven (ac to dc) } \\
\text { Fails to start }\end{array}$ & MDG-FS.E & $1.0 \mathrm{E} \cdot 5 / \mathrm{h}$ & 10 \\
\hline Fails to run & MDG-FR-E & $3.0 \mathrm{E} \cdot 5 / \mathrm{h}$ & 10 \\
\hline $\begin{array}{l}\text { Gas-Turbine-Driven } \\
\text { Fails to start }\end{array}$ & GTG-FS-E & $3.0 \mathrm{E}-2 / \mathrm{d}$ & 10 \\
\hline Fails to $\mathrm{Nm}$ & GTG-FR-E & $3.0 \mathrm{E}-4 / \mathrm{h}$ & 10 \\
\hline $\begin{array}{l}\text { Hydro-Turbine-Driven } \\
\text { Fails to start }\end{array}$ & HDG-FS-E & $3.0 \mathrm{E}-3 / \mathrm{d}$ & 10 \\
\hline Fails to run & HDG-FR-E & $3.0 \mathrm{E}-4 / \mathrm{h}$ & 10 \\
\hline $\begin{array}{c}\text { Battery } \\
\text { Failure }\end{array}$ & BAT-FA-E & $1.0 \mathrm{E}-5 / \mathrm{h}$ & 3 \\
\hline $\begin{array}{l}\text { Charger } \\
\text { Rectifier } \\
\text { Failure }\end{array}$ & RCT-FA-E & $1.0 \mathrm{E}-5 / \mathrm{h}$ & 3 \\
\hline $\begin{array}{l}\text { Bus } \\
\text { Metal-Enclosed } \\
\text { Failure }\end{array}$ & BUM-FA-E & $1.0 \mathrm{E}-7 / \mathrm{h}$ & 5 \\
\hline $\begin{array}{l}\text { Bare } \\
\text { Failure }\end{array}$ & BUB-FA-E & $1.0 \mathrm{E}-6 / \mathrm{h}$ & 10 \\
\hline $\begin{array}{l}\text { Cable/Joiny } \\
\text { Termination/Jumper } \\
\text { Cable (Copper, 1000fr) } \\
\text { Failure } \\
\end{array}$ & CBLFA-E & $3.0 \mathrm{E}-6 / \mathrm{h}$ & 3 \\
\hline $\begin{array}{l}\text { Joint (Copper) } \\
\text { Failure } \\
\end{array}$ & JNT-FA-E & $3.0 \mathrm{E} \cdot 6 / \mathrm{h}$ & 30 \\
\hline $\begin{array}{l}\text { Termination (Copper) } \\
\text { Failure }\end{array}$ & TMN-FA-E & $3.0 \mathrm{E}-7 / \mathrm{h}$ & 10 \\
\hline $\begin{array}{l}\text { Jumper (Power) } \\
\text { Failure }\end{array}$ & JPR-FA-E & $5.0 \mathrm{E} \cdot 6 / \mathrm{h}$ & 10 \\
\hline $\begin{array}{l}\text { Circuit Breaker } \\
\text { General } \\
\text { Fails to oncn/close }\end{array}$ & $\begin{array}{l}\text { CBR-CC.E or } \\
\text { CBR-OO-E or } \\
\text { CBR-NR-E } \\
\end{array}$ & $5.0 \mathrm{E}-4 / \mathrm{d}$ & 5 \\
\hline Spurious operation & $\begin{array}{l}\text { CBR-CO-E or } \\
\text { CBR-OC-E or } \\
\text { CBR-SO-E } \\
\end{array}$ & $3.0 \mathrm{E}-7 / \mathrm{h}$ & 10 \\
\hline $\begin{array}{l}\text { Reactor Trip } \\
\text { Fails to open }\end{array}$ & RTB-CC.E & $5.0 \mathrm{E}-3 / \mathrm{d}$ & 5 \\
\hline Spurious operation & RTB-CO-E & $3.0 \mathrm{E}-6 / \mathrm{h}$ & 10 \\
\hline $\begin{array}{l}\text { Relay } \\
\text { Protective } \\
\text { Fails to open/close }\end{array}$ & $\begin{array}{c}\text { RLP-CC-E or } \\
\text { RLP-OO-E or } \\
\because \mathrm{E}\end{array}$ & $1.0 \mathrm{E}-3 / \mathrm{d}$ & 10 \\
\hline Spurious operation & $\begin{array}{l}\text { RLP-CO-E or } \\
\text { RLP.OC-E or } \\
\text { RLP-SO-E }\end{array}$ & $1.0 E-7 / \mathrm{h}$ & 10 \\
\hline $\begin{array}{l}\text { Control } \\
\text { Fails to oper/close }\end{array}$ & $\begin{array}{l}\text { RLC-CC-E or } \\
\text { RLC-OO-E or } \\
\text { RLC-NR-E }\end{array}$ & $1.0 \mathrm{E}-4 / \mathrm{d}$ & 10 \\
\hline
\end{tabular}

Note: Modification of the recommended mean for hourly failure rates may be appropriate for components subjected to hostile radioactive or chemical environments. 


\begin{tabular}{|c|c|c|c|}
\hline \multirow[t]{2}{*}{ System/Component/Failure Mode } & \multirow[t]{2}{*}{ Identifier } & \multicolumn{2}{|c|}{$\begin{array}{l}\text { Recommended Failure Rate } \\
\text { Distribution (logno.mal) }\end{array}$} \\
\hline & & Mean & Error Factor ${ }^{a}$ \\
\hline Spurious operation & $\begin{array}{l}\text { RLC-CO-E or } \\
\text { RLC-OC-E or } \\
\text { RLC-SO-E }\end{array}$ & $3.0 \mathrm{E}-7 / \mathrm{h}$ & 30 \\
\hline $\begin{array}{l}\text { Bistable } \\
\text { Fails to open/close }\end{array}$ & $\begin{array}{l}\text { BIS-CC-E or } \\
\text { BIS-OO-E or } \\
\text { BIS-NR-E }\end{array}$ & $1.0 \mathrm{E}-5 / \mathrm{d}$ & 10 \\
\hline Spurious operation & $\begin{array}{l}\text { BIS-CO-E or } \\
\text { BIS-OC-E or } \\
\text { BIS-SO-E }\end{array}$ & $3.0 \mathrm{E}-7 / \mathrm{h}$ & 10 \\
\hline $\begin{array}{l}\text { Switch } \\
\text { Push-Button (Manual) } \\
\text { Fails to open/close }\end{array}$ & $\begin{array}{l}\text { XSP-CC-E or } \\
\text { XSP-OO-E or } \\
\text { XSP-NR-E }\end{array}$ & $1.0 \mathrm{E} \cdot 6 / \mathrm{h}$ & 10 \\
\hline Spurious operation & $\begin{array}{l}\text { XSP-CO-E or } \\
\text { XSP-OC-E or } \\
\text { XSP-SO-E }\end{array}$ & $1.0 \mathrm{E}-6 / \mathrm{h}$ & 10 \\
\hline $\begin{array}{l}\text { Rotary (Manual) } \\
\text { Fails to open/close }\end{array}$ & $\begin{array}{l}\text { XSR-CC-E or } \\
\text { XSR-OO-E or } \\
\text { XSR-NR-E }\end{array}$ & $5.0 \mathrm{E}-8 / \mathrm{h}$ & 10 \\
\hline Spurious operation & $\begin{array}{l}\text { XSR-CO-E or } \\
\text { XSR-OC-E or } \\
\text { XSR-SO-E }\end{array}$ & $5.0 \mathrm{E}-7 / \mathrm{h}$ & 10 \\
\hline $\begin{array}{l}\text { Key-Operated (Manual) } \\
\text { Fails to open/close }\end{array}$ & $\begin{array}{l}\text { XSK-CC-E or } \\
\text { XSK-OO-E or } \\
\text { XSK-NR-E }\end{array}$ & $3.0 \mathrm{E}-7 / \mathrm{h}$ & 10 \\
\hline Spurious operation & $\begin{array}{l}\text { XSK-CO-E or } \\
\text { XSK-OC-E or } \\
\text { XSK-SO-E }\end{array}$ & $1.0 \mathrm{E}-6 / \mathrm{h}$ & 10 \\
\hline $\begin{array}{l}\text { Automatic-Transfer } \\
\text { Fails to open/close }\end{array}$ & $\begin{array}{l}\text { ATS-CC-E or } \\
\text { ATS-OO-E or } \\
\text { ATS-NR-E }\end{array}$ & $1.0 \mathrm{E}-6 / \mathrm{h}$ & 10 \\
\hline Spurious operation & $\begin{array}{l}\text { ATS-CO-E or } \\
\text { ATS-OC-E or } \\
\text { ATS-SO-E }\end{array}$ & $1.0 \mathrm{E}-6 / \mathrm{h}$ & 10 \\
\hline $\begin{array}{l}\text { Limit } \\
\text { Fails to open/close }\end{array}$ & $\begin{array}{l}\text { LMS-CC-E or } \\
\text { LMS-OO-E or } \\
\text { LMS-NR-E }\end{array}$ & $1.0 \mathrm{E}-6 / \mathrm{h}$ & 10 \\
\hline Spurious operation & $\begin{array}{l}\text { LMS-CO-E or } \\
\text { LMS-OC-E or } \\
\text { LMS-SO-E }\end{array}$ & $1.0 \mathrm{E}-6 / \mathrm{h}$ & 5 \\
\hline $\begin{array}{l}\text { Fuse } \\
\text { Fail to open }\end{array}$ & FUS-CC-E & $1.0 \mathrm{E}-7 / \mathrm{h}$ & 10 \\
\hline Premature opening & FUS-SO-E & $1.0 \mathrm{E}-8 / \mathrm{h}$ & 10 \\
\hline $\begin{array}{l}\text { Inverter } \\
\text { Failure }\end{array}$ & INV-FA-E & $1.0 \mathrm{E}-5 / \mathrm{h}$ & 3 \\
\hline $\begin{array}{l}\text { Motor } \\
\text { AC } \\
\text { Fails to start }\end{array}$ & MRA-FS-E & $3.0 \mathrm{E}-4 / \mathrm{d}$ & 3 \\
\hline Fails to nu & MRA-FR-E & $5.0 \mathrm{E}-6 / \mathrm{h}$ & $\overline{3}$ \\
\hline $\begin{array}{l}\text { DC } \\
\text { Fails to start }\end{array}$ & MRD-FS-E & $3.0 \mathrm{E}-4 / \mathrm{d}$ & 3 \\
\hline
\end{tabular}

Notc: Modification of the recommended mean for hourly failure rates may be appropriate for components subjected to hostile radioactive or chemical environments. 
WSRC-TR-93-262

\begin{tabular}{||c|l|l|l||}
\hline \multicolumn{3}{|c|}{ Table 1e. Electrical distribution system recommended generic failure rates (cont'd). } \\
\hline System/Component/Failure Mode & Identifier & \multicolumn{1}{|c|}{$\begin{array}{c}\text { Recommended Failure Rate } \\
\text { Distribution (lognormal) }\end{array}$} \\
\cline { 2 - 4 } & & Mean & \multicolumn{1}{|c|}{ Error Factor } \\
\hline Fails to nu & MRD-FR-E & $1.0 \mathrm{E}-5 / \mathrm{h}$ & 3 \\
\hline $\begin{array}{c}\text { Synchro } \\
\text { Failure }\end{array}$ & SYN-FA-E & $1.0 \mathrm{E}-5 / \mathrm{h}$ & 10 \\
\hline $\begin{array}{c}\text { Transformer } \\
\text { Power } \\
\text { Failure }\end{array}$ & TFP-FA-E & $1.0 \mathrm{E}-6 / \mathrm{h}$ & 10 \\
\hline $\begin{array}{c}\text { Instrumentation/Control } \\
\text { Failure }\end{array}$ & TFI-FA-E & $1.0 \mathrm{E}-6 / \mathrm{h}$ & 10 \\
\hline
\end{tabular}

a. Error factor is the 95 th percentile/50th percentile.

Note: Modification of the recommended mean for hourly failure rates may be appropriate for components subjected to hostile radioactive or chemical environments. 


\begin{tabular}{|c|c|c|c|}
\hline \multirow[t]{2}{*}{ System/Component/Failure Mode } & \multirow[t]{2}{*}{ Identifier } & \multicolumn{2}{|c|}{$\begin{array}{l}\text { Recommended Failure Rate } \\
\text { Distribution (lognormal) }\end{array}$} \\
\hline & & Mean & Error Factor ${ }^{\mathrm{a}}$ \\
\hline $\begin{array}{l}\text { Instrumentation and Control } \\
\text { Alarm/Annunciator } \\
\text { Fails to alarm }\end{array}$ & ALR-NR-I & $3.0 \mathrm{E}-5 / \mathrm{h}$ & 10 \\
\hline Spurious operation & ALR-SO-I & $5.0 \mathrm{E}-6 / \mathrm{h}$ & 10 \\
\hline $\begin{array}{l}\text { Sensor/Transmitter/ } \\
\text { Transducer/Process Switch } \\
\text { Temperature } \\
\text { Failure } \\
\end{array}$ & TST-FA-I & $1.0 \mathrm{E}-6 / \mathrm{h}$ & 3 \\
\hline $\begin{array}{l}\text { Pressure } \\
\text { Failure }\end{array}$ & PST-FA-I & $1.0 \mathrm{E}-6 / \mathrm{h}$ & 3 \\
\hline $\begin{array}{l}\text { Differential Pressure } \\
\text { Failure }\end{array}$ & DPS-FA-I & $3.0 \mathrm{E}-6 / \mathrm{h}$ & 10 \\
\hline $\begin{array}{l}\text { Flow } \\
\text { Failure }\end{array}$ & FST-FA-I & $3.0 \mathrm{E}-6 / \mathrm{h}$ & 3 \\
\hline $\begin{array}{l}\text { Level } \\
\text { Failure } \\
\end{array}$ & LST-FA-I & $5.0 \mathrm{E}-7 / \mathrm{h}$ & 3 \\
\hline $\begin{array}{r}\text { Humidity } \\
\text { Failure }\end{array}$ & UST-FA-I & $1.0 \mathrm{E}-5 / \mathrm{h}$ & 10 \\
\hline $\begin{array}{l}\mathrm{pH} \\
\text { Failure } \\
\end{array}$ & HST-FA-I & $5.0 \mathrm{E}-7 / \mathrm{h}$ & 5 \\
\hline $\begin{array}{l}\text { Oxygen Concentration } \\
\text { Failure }\end{array}$ & OXC-FA-I & $1.0 \mathrm{E}-5 / \mathrm{h}$ & 10 \\
\hline $\begin{array}{l}\mathrm{CO}_{2} \text { Concentration } \\
\text { Failure }\end{array}$ & COC-FA-I & $1.0 \mathrm{E}-4 / \mathrm{h}$ & 10 \\
\hline $\begin{array}{l}\text { Hydrogen Concentration } \\
\text { Failure }\end{array}$ & HYC-FA-I & $1.0 \mathrm{E}-5 / \mathrm{h}$ & 3 \\
\hline $\begin{array}{l}\text { Nitrogen Concentration } \\
\text { Failure } \\
\end{array}$ & NIC-FA-I & $1.0 \mathrm{E}-5 / \mathrm{h}$ & 3 \\
\hline $\begin{array}{l}\text { Hydrocarbon Concentration } \\
\text { Failure }\end{array}$ & HCC-FA-I & $1.0 \mathrm{E}-5 / \mathrm{h}$ & 3 \\
\hline $\begin{array}{l}\text { Helium Concentration } \\
\text { Failure } \\
\end{array}$ & HEC-FA-I & $1.0 \mathrm{E}-5 / \mathrm{h}$ & 3 \\
\hline $\begin{array}{l}\text { Speed } \\
\text { Failure } \\
\end{array}$ & SST-FA-I & $1.0 \mathrm{E}-6 / \mathrm{h}$ & 10 \\
\hline $\begin{array}{c}\text { Seismic } \\
\text { Failure } \\
\end{array}$ & SET.FA-I & $1.0 \mathrm{E}-6 / \mathrm{h}$ & 5 \\
\hline $\begin{array}{c}\text { Radiation } \\
\text { Failure } \\
\end{array}$ & RST-FA-I & $5.0 \mathrm{E}-6 / \mathrm{h}$ & 5 \\
\hline $\begin{array}{c}\text { Indicator } \\
\text { Failure } \\
\end{array}$ & IND-FA-I & $1.0 \mathrm{E}-5 / \mathrm{h}$ & 10 \\
\hline $\begin{array}{l}\text { Amplifier } \\
\text { Failure } \\
\end{array}$ & AMP-FA-I & $5.0 \mathrm{E}-6 / \mathrm{h}$ & 10 \\
\hline $\begin{array}{l}\text { Modifier/Signal Conditioner } \\
\text { Failure }\end{array}$ & SCR-FA-I & $3.0 \mathrm{E}-7 / \mathrm{h}$ & 3 \\
\hline $\begin{array}{l}\text { Logic Module } \\
\text { Failure }\end{array}$ & LOG-FA-I & $3.0 \mathrm{E}-6 / \mathrm{h}$ & 5 \\
\hline $\begin{array}{c}\text { Recorder } \\
\text { Failure } \\
\end{array}$ & REC-FA-I & $3.0 \mathrm{E}-5 / \mathrm{h}$ & 30 \\
\hline $\begin{array}{c}\begin{array}{c}\text { Sampler } \\
\text { Failure }\end{array} \\
\end{array}$ & SAM-FA-I & $1.0 \mathrm{E}-5 / \mathrm{h}$ & 10 \\
\hline $\begin{array}{c}\text { Analyzer } \\
\text { Failure } \\
\end{array}$ & ANA-FA-I & $5.0 \mathrm{E}-6 / \mathrm{h}$ & 10 \\
\hline $\begin{array}{c}\text { Timer } \\
\text { Failure }\end{array}$ & TMR-FA-I & $5.0 \mathrm{E}-6 / \mathrm{h}$ & 10 \\
\hline
\end{tabular}

Note: Modification of the recommended mean for hourly failure rates may be appropriate for components subjected to hostile radioactive or chemical environments. 


\begin{tabular}{||c|c|c|c||}
\hline Table If. Instrumentation and control system recommended generic failure rates. \\
\hline System/Component/Failure Mode & \multicolumn{1}{|c|}{ Identifier } & \multicolumn{2}{|c|}{$\begin{array}{c}\text { Recommended Failure Rate } \\
\text { Distribution (lognormal) }\end{array}$} \\
\cline { 2 - 4 } & & \multicolumn{1}{|c|}{ Mean } & \multicolumn{1}{|c|}{ Error Factor ${ }^{\mathrm{a}}$} \\
\hline $\begin{array}{c}\text { Gas Chromatograph } \\
\text { Failure }\end{array}$ & GCR-FA-I & $5.0 \mathrm{E}-5 / \mathrm{h}$ & 10 \\
\hline $\begin{array}{c}\text { Voltage Regulator } \\
\text { Failure }\end{array}$ & VRG-FA-I & $3.0 \mathrm{E}-6 / \mathrm{h}$ & 10 \\
\hline $\begin{array}{c}\text { Transmitter } \\
\text { Failure }\end{array}$ & TRM-FA-I & $3.0 \mathrm{E}-6 / \mathrm{h}$ & 10 \\
\hline $\begin{array}{c}\text { Transducer } \\
\text { Failure }\end{array}$ & TRD-FA-I & $1.0 \mathrm{E}-6 / \mathrm{h}$ & 10 \\
\hline $\begin{array}{c}\text { Programmable Logic Controller } \\
\text { Failure }\end{array}$ & PLC-FA-I & $3.0 \mathrm{E}-5 / \mathrm{h}$ & 10 \\
\hline
\end{tabular}

a. Error factor is the 95 th percentile/50th percentile.

Notc: Modification of the recommended mean fo: hourly failure rates may be appropriate for components subjected to hostile radioactive or chemical environments. 
Table 2. Data sources used in generic data base development.

\section{Category 1}

\section{Big Rock Point PRA ${ }^{12}$}

\section{Oconee-3 PRA 13}

Zion PRA ${ }^{14}$

Indian Point PRA 15

Reliability data from Swedish nuclear power plants ${ }^{16}$

Millstone 1 PRA 17

Connecticut Yankee PRA 18

B\&W nuclear power plant (name withneld) ${ }^{a}$

European nuclear power plant (name withheld) PRAa

GE nuclear power plant (name withheld) ${ }^{a}$

Westinghouse nuclear power plant (name withheld) ${ }^{a}$

7 other nuclear power plant (names withheld) PRAs ${ }^{a}$

Savannah River Site reactors 3,4 , and 5

\section{Category 2}

Licensee event report (LER) survey of pumps at nuclear power plants ${ }^{19}$

LER survey of valves 20

LER survey of inverters 21

LER survey of selected instrumentation and control components 22

In-plant Reliability Data Base (IPRD) survey of pumps ${ }^{23}$

IPRD survey of valves 24

IPRD survey of diesel generators, batteries, chargers, and inverters 25

Nuclear Safety Analysis Center (NSAC) survey of diesel generators 26

Pipe break survey27

LER survey of pipes, valves, pumps, etc. 28

Nonelectronic Parts Reliability Data (military) ${ }^{6}$

OREDA data for offshore oil drilling components 7

Chemical processing plant 2

Tritium handling facility 8,9 , and 10

Liquid natural gas plant components 11

Category 3

WASH-140029

Center for Chemical Process Safety 30

IEEE Std 500-198431

Natural gas facility components 32

Gasification-combined-cycle power generation components 33

Coal-fired power generation components 34

Generic data base for nuclear reactor components 35

a. See Reference 1 for more details on these studies. 


\begin{tabular}{|c|c|c|c|}
\hline \multirow[t]{2}{*}{ System/Component/Failure Mode } & \multicolumn{3}{|c|}{ Failure Data (Category 1 Sources) ${ }^{2}$} \\
\hline & NUCLARRI & $\begin{array}{c}\text { SRS- } \\
\text { REACTORS }\end{array}$ & $\begin{array}{c}\text { Aggregated } \\
\text { Results }^{\mathrm{b}} \\
\end{array}$ \\
\hline $\begin{array}{l}\text { Water } \\
\text { Valve (Standby or Safety) } \\
\text { Manual } \\
\text { Fails to open/close }\end{array}$ & $\begin{array}{l}38 / 8.8 \mathrm{E}+4 \mathrm{~d}, \\
7 / 2.1 \mathrm{E}+8 \mathrm{~h} \\
\text { [1] }\end{array}$ & $\begin{array}{l}0 / 7.1 \mathrm{E}+3 \mathrm{~d}, \\
0 / 1.3 \mathrm{E}+4 \mathrm{~d}, \\
0 / 8.4 \mathrm{E}+2 \mathrm{~d}, \\
0 / 5.8 \mathrm{E}+2 \mathrm{~d} \\
{[1]}\end{array}$ & $\begin{array}{l}3.5 \mathrm{E}-4 / \mathrm{d}(18), \\
3.3 \mathrm{E}-8 \mathrm{~h}(88)\end{array}$ \\
\hline Plugs & & $\begin{array}{l}0 / 4.6 \mathrm{E}+5 \mathrm{~h}, \\
0 / 7.6 \mathrm{E}+3 \mathrm{~h}, \\
0 / 1.5 \mathrm{E}+3 \mathrm{~h} \\
{[2]}\end{array}$ & $\begin{array}{l}1.1 \mathrm{E}-6 / \mathrm{h}(10) \\
{[1]}\end{array}$ \\
\hline Leakage (internal) & & [3] & $\begin{array}{l}5.7 \mathrm{E}-3 / \mathrm{h}(10) \\
{[1]}\end{array}$ \\
\hline Rupture (internal) & & [3] & \\
\hline \multicolumn{4}{|l|}{ Leakage (external) } \\
\hline \multicolumn{4}{|l|}{ Ruptare (external) } \\
\hline $\begin{array}{l}\text { Check } \\
\text { Fails to open }\end{array}$ & $\begin{array}{l}1 / 2.5 E+4 d \\
{[2]}\end{array}$ & $\begin{array}{l}1 / 3.3 \mathrm{E}+3 \mathrm{~d}, \\
0 / 3.6 \mathrm{E}+2 \mathrm{~d}, \\
0 / 4.7 \mathrm{E}+2 \mathrm{~d}, \\
0 / 2.2 \mathrm{E}+2 \mathrm{~d}, \\
0 / 2.7 \mathrm{E}+3 \mathrm{~d}, \\
{[4]}\end{array}$ & $\begin{array}{l}6.0 \mathrm{E}-5 / \mathrm{d}(10) \\
{[2\}}\end{array}$ \\
\hline Fails to close & $\begin{array}{l}18 / 1.2 \mathrm{E}+4 \mathrm{~d} \\
{[3]}\end{array}$ & [4] & $\begin{array}{l}9.8 \mathrm{E}-4 / \mathrm{d}(4.6) \\
{[2]}\end{array}$ \\
\hline \multicolumn{4}{|l|}{ Plugs } \\
\hline Leakage (internal) & $\begin{array}{l}36 / 1.2 \mathrm{E}+7 \mathrm{~h} \\
{[4]}\end{array}$ & $\begin{array}{l}0 / 4.1 \mathrm{E}+7 \mathrm{~h} \\
{[5]}\end{array}$ & $6.8 \mathrm{E}-7 / \mathrm{h}(63)$ \\
\hline Rupture (intemal) & [4] & [5] & \\
\hline \multicolumn{4}{|l|}{ Leakage (external) } \\
\hline \multicolumn{4}{|l|}{ Rupture (external) } \\
\hline $\begin{array}{l}\text { Motor-Operated } \\
\text { Fails to open/close }\end{array}$ & $\begin{array}{l}390 / 1.2 \mathrm{E}+5 \mathrm{~d} \\
15 / 3.1 \mathrm{E}+6 \mathrm{~h} \\
{[5]}\end{array}$ & $\begin{array}{l}29 / 9.2 \mathrm{E}+3 \mathrm{~d} \\
10 / 7.5 \mathrm{E}+2 \mathrm{~d} \\
29 / 5.3 \mathrm{E}+3 \mathrm{~d} \\
4 / 1.8 \mathrm{E}+3 \mathrm{~d} \\
{[6]}\end{array}$ & $\begin{array}{l}3.3 \mathrm{E}-3 / \mathrm{d}(6.3), \\
4.8 \mathrm{E}-6 / \mathrm{h}(9.6)\end{array}$ \\
\hline
\end{tabular}




\begin{tabular}{|c|c|c|c|}
\hline \multirow[t]{2}{*}{ System/Component/Failure Mode } & \multicolumn{3}{|c|}{ Failure Data (Category 1 Sources) ${ }^{a}$} \\
\hline & NUCLARR1 & $\begin{array}{c}\text { SRS- } \\
\text { REACTORS }\end{array}$ & $\begin{array}{c}\text { Aggregated } \\
\text { Results }\end{array}$ \\
\hline Spurious operation & $\begin{array}{l}1 / 2.1 E+7 h \\
{[6]}\end{array}$ & $\begin{array}{l}2 / 1.3 \mathrm{E}+7 \mathrm{~h}, \\
0 / 2.5 \mathrm{E}+6 \mathrm{~h}, \\
7 / 4.4 \mathrm{E}+6 \mathrm{~h}, \\
0 / 1.9 \mathrm{E}+6 \mathrm{~h} \\
{[7]}\end{array}$ & $2.3 \mathrm{E}-7 / \mathrm{h}(7.2)$ \\
\hline Plugs & & $\begin{array}{l}1 / 1.8 \mathrm{E}+7 \mathrm{~h}, \\
0 / 2.5 \mathrm{E}+6 \mathrm{~h}, \\
0 / 4.4 \mathrm{E}+6 \mathrm{~h}, \\
0 / 1.9 \mathrm{E}+6 \mathrm{~h} \\
{[8]}\end{array}$ & $5.6 \mathrm{E}-8 / \mathrm{h}(10)$ \\
\hline Leakage (intemal) & & $\begin{array}{l}1 / 1.8 \mathrm{E}+6 \mathrm{~h} \\
{[9]}\end{array}$ & $8.3 \mathrm{E}-7 / \mathrm{h}(10)$ \\
\hline Rupture (internal) & & [9] & \\
\hline \multicolumn{4}{|l|}{ Leakage (external) } \\
\hline \multicolumn{4}{|l|}{ Rupture (extemal) } \\
\hline $\begin{array}{l}\text { Air-Operated } \\
\text { Fails to open/close }\end{array}$ & $\begin{array}{l}110 / 9.4 \mathrm{E}+4 \mathrm{~d}, \\
2 / 4.8 \mathrm{E}+6 \mathrm{~h} \\
{[7]}\end{array}$ & $\begin{array}{l}4 / 2.9 \mathrm{E}+3 \mathrm{~d}, \\
0 / 8.8 \mathrm{E}+2 \mathrm{~d}, \\
1 / 6.0 \mathrm{E}+2 \mathrm{~d} \\
{[10]}\end{array}$ & $\begin{array}{l}1.2 \mathrm{E}-3 / \mathrm{d}(21), \\
5.2 \mathrm{E}-7 / \mathrm{h}(10)\end{array}$ \\
\hline Spurious operation & $\begin{array}{l}13 / 6.2 \mathrm{E}+6 \mathrm{~h} \\
{[8]}\end{array}$ & $\begin{array}{l}0 / 4.0 \mathrm{E}+6 \mathrm{~h}, \\
0 / 5.0 \mathrm{E}+5 \mathrm{~h}, \\
0 / 1.5 \mathrm{E}+3 \mathrm{~h} \\
{[11]}\end{array}$ & $1.2 \mathrm{E}-6 / \mathrm{h}(5.4)$ \\
\hline Plugs & & $\begin{array}{l}0 / 4.0 \mathrm{E}+6 \mathrm{~h}, \\
0 / 5.0 \mathrm{E}+5 \mathrm{~h}, \\
0 / 2.6 \mathrm{E}+1 \mathrm{~h} \\
{[12]}\end{array}$ & $\begin{array}{l}1.1 \mathrm{E}-7 / \mathrm{h}(10) \\
{[1]}\end{array}$ \\
\hline Leakage (internal) & $\begin{array}{l}8 / 5.3 \mathrm{E}+6 \mathrm{~h} \\
{[9]}\end{array}$ & & $1.6 \mathrm{E}-6 / \mathrm{h}(10)$ \\
\hline Rupture (intemal) & [9] & & \\
\hline \multicolumn{4}{|l|}{ Leakage (external) } \\
\hline \multicolumn{4}{|l|}{ Rupture (external) } \\
\hline $\begin{array}{l}\text { Solenoid-Operated } \\
\text { Fails to open/close }\end{array}$ & $\begin{array}{l}2 / 3.5 \mathrm{E}+3 \mathrm{~d} \\
16 / 2.3 \mathrm{E}+7 \mathrm{~h} \\
{[10]}\end{array}$ & $\begin{array}{l}2 / 2.3 \mathrm{E}+3 \mathrm{~d} \\
{[13]}\end{array}$ & $\begin{array}{l}7.8 \mathrm{E}-4 / \mathrm{d}(10) \\
7.1 \mathrm{E}-7 / \mathrm{h}(10)\end{array}$ \\
\hline
\end{tabular}


WSRC-TR-93-262

\begin{tabular}{|c|c|c|c|}
\hline \multirow[t]{2}{*}{ System/Componen//Failure Mode } & \multicolumn{3}{|c|}{ Failure Data (Category 1 Sources) ${ }^{\mathrm{a}}$} \\
\hline & NUCLARR1 & $\begin{array}{c}\text { SRS- } \\
\text { REACTORS } \\
\end{array}$ & $\begin{array}{c}\text { Aggregated } \\
\text { Results }^{b}\end{array}$ \\
\hline Spurious operation & $\begin{array}{l}0 / 8.1 \mathrm{E}+5 \mathrm{~h} \\
{[11]}\end{array}$ & $\begin{array}{l}0 / 1.5 \mathrm{E}+3 \mathrm{~h} \\
{[14]}\end{array}$ & $\begin{array}{l}6.1 \mathrm{E}-7 / \mathrm{h}(10) \\
\text { [1] }\end{array}$ \\
\hline Plugs & & $\begin{array}{l}0 / 1.5 \mathrm{E}+3 \mathrm{~h} \\
{[15]}\end{array}$ & $\begin{array}{l}3.3 \mathrm{E}-4 / \mathrm{h}(10) \\
{[1]}\end{array}$ \\
\hline \multicolumn{4}{|l|}{ Leakage (internal) } \\
\hline \multicolumn{4}{|l|}{ Rupture (internal) } \\
\hline \multicolumn{4}{|l|}{ Leakage (external) } \\
\hline \multicolumn{4}{|l|}{ Rupture (external) } \\
\hline $\begin{array}{l}\text { Safety/Relief } \\
\text { Fails to open }\end{array}$ & $\begin{array}{l}15 / 5.4 \mathrm{E}+3 \mathrm{~d}, \\
0 / 5.9 \mathrm{E}+6 \mathrm{~h} \\
{[12]}\end{array}$ & & $\begin{array}{l}2.7 \mathrm{E}-3 / \mathrm{d}(3.3), \\
8.5 \mathrm{E}-8 / \mathrm{h}(10) \\
{[1]}\end{array}$ \\
\hline Fails to reclose & $\begin{array}{l}47 / 2.0 \mathrm{E}+4 \mathrm{~d} \\
{[13]}\end{array}$ & & $2.3 \mathrm{E}-3 / \mathrm{d}(1.7)$ \\
\hline \multicolumn{4}{|l|}{ Leakage (internal) } \\
\hline \multicolumn{4}{|l|}{ Rupture (internal) } \\
\hline \multicolumn{4}{|l|}{ Leakage (extemal) } \\
\hline \multicolumn{4}{|l|}{ Rupture (external) } \\
\hline $\begin{array}{l}\text { Vacuum-Breaker } \\
\text { Fails to open }\end{array}$ & $\begin{array}{l}0 / 4.5 \mathrm{E}+1 \mathrm{~d}, \\
0 / 1.1 \mathrm{E}+3 \mathrm{~h} \\
{[14]}\end{array}$ & & $\begin{array}{l}1.1 \mathrm{E}-2 / \mathrm{d}(10), \\
4.4 \mathrm{E}-4 / \mathrm{h}(10) \\
\text { [1] }\end{array}$ \\
\hline \multicolumn{4}{|l|}{ Fails to reclose } \\
\hline \multicolumn{4}{|l|}{ Leakage (intemal) } \\
\hline \multicolumn{4}{|l|}{ Rupture (intemal) } \\
\hline \multicolumn{4}{|l|}{ Leakage (external) } \\
\hline \multicolumn{4}{|l|}{ Rupture (external) } \\
\hline $\begin{array}{l}\text { Explosive } \\
\text { Fails to open }\end{array}$ & & $\begin{array}{l}0 / 2.7 E+1 d \\
{[16]}\end{array}$ & $\begin{array}{l}1.8 \mathrm{E}-2 / \mathrm{d}(10) \\
{[1]}\end{array}$ \\
\hline \multicolumn{4}{|l|}{ Leakage (internal) } \\
\hline Rupture (internal) & & & \\
\hline Leakage (external) & & & \\
\hline
\end{tabular}




\begin{tabular}{|c|c|c|c|}
\hline \multirow[t]{2}{*}{ System/Component/Failure Mode } & \multicolumn{3}{|c|}{ Failure Data (Category 1 Sources) ${ }^{\mathrm{a}}$} \\
\hline & NUCLARR1 & $\begin{array}{c}\text { SRS- } \\
\text { REACTORS }\end{array}$ & $\begin{array}{c}\text { Aggregated } \\
\text { Results } \\
\text { b }\end{array}$ \\
\hline \multicolumn{4}{|l|}{ Rupture (external) } \\
\hline \multicolumn{4}{|l|}{$\begin{array}{l}\text { Valve (Control) } \\
\text { Motor-Operated } \\
\text { Fails open }\end{array}$} \\
\hline \multicolumn{4}{|l|}{ Fails closed } \\
\hline \multicolumn{4}{|l|}{ Fails to respond } \\
\hline \multicolumn{4}{|l|}{ Plugs. } \\
\hline \multicolumn{4}{|l|}{ Leakage (external) } \\
\hline \multicolumn{4}{|l|}{ Rupture (external) } \\
\hline \multicolumn{4}{|l|}{$\begin{array}{l}\text { Air-Operated } \\
\text { Fails open }\end{array}$} \\
\hline \multicolumn{4}{|l|}{ Fails closed } \\
\hline \multicolumn{4}{|l|}{ Fails to respond } \\
\hline \multicolumn{4}{|l|}{ Plugs } \\
\hline \multicolumn{4}{|l|}{ Leakage (extemal) } \\
\hline \multicolumn{4}{|l|}{ Rupture (external) } \\
\hline \multicolumn{4}{|l|}{$\begin{array}{l}\text { Solenoid-Operated } \\
\text { Fails open }\end{array}$} \\
\hline \multicolumn{4}{|l|}{$\begin{array}{l}\text { Pauls open } \\
\text { Fails closed }\end{array}$} \\
\hline \multicolumn{4}{|l|}{ Fails to respond } \\
\hline \multicolumn{4}{|l|}{ Plugs } \\
\hline \multicolumn{4}{|l|}{ Leakage (extemal) } \\
\hline \multicolumn{4}{|l|}{ Rupture (external) } \\
\hline $\begin{array}{l}\text { Pump } \\
\text { Motor-Driven } \\
\text { Fails to start }\end{array}$ & $\begin{array}{l}137 / 4.9 \mathrm{E}+4 \mathrm{~d} \\
{[15]}\end{array}$ & $\begin{array}{l}25 / 2.1 \mathrm{E}+3 \mathrm{~d} \\
3 / 2.6 \mathrm{E}+2 \mathrm{~d} \\
42 / 3.7 \mathrm{E}+3 \mathrm{~d}, \\
6 / 2.4 \mathrm{E}+3 \mathrm{~d} \\
{[17]}\end{array}$ & $3.7 \mathrm{E}-3 / \mathrm{d}(4.7)$ \\
\hline
\end{tabular}


WSRC-TR-93-262

\begin{tabular}{|c|c|c|c|}
\hline \multirow[t]{2}{*}{ System/Component/Failure Mode } & \multicolumn{3}{|c|}{ Failure Data (Category 1 Sources) ${ }^{\mathbf{a}}$} \\
\hline & NUCLARR1 & $\begin{array}{c}\text { SRS- } \\
\text { REACTORS } \\
\end{array}$ & $\begin{array}{c}\text { Aggregated } \\
\text { Resulss }^{\mathrm{b}} \\
\end{array}$ \\
\hline Fails to run & $\begin{array}{l}2167.5 \mathrm{E}+6 \mathrm{~h} \\
{[16]}\end{array}$ & $\begin{array}{l}23 / 1.7 \mathrm{E}+6 \mathrm{~h}, \\
0 / 4.1 \mathrm{E}+2 \mathrm{~h}, \\
0 / 3.1 \mathrm{E}+4 \mathrm{~h}, \\
19 / 2.6 \mathrm{E}+6 \mathrm{~h} \\
{[18]}\end{array}$ & $2.2 \mathrm{E}-5 / \mathrm{h}(17)$ \\
\hline \multicolumn{4}{|l|}{ Overiped } \\
\hline Falls to stop & & $\begin{array}{l}5 / 2.6 E+2 d \\
5 / 2.4 E+3 d \\
{[19]}\end{array}$ & $3.8 \mathrm{E}-3 / \mathrm{d}(4.4)$ \\
\hline \multicolumn{4}{|l|}{ Leakage (external) } \\
\hline \multicolumn{4}{|l|}{ Rupture (extemal) } \\
\hline $\begin{array}{l}\text { Turbine-Driven } \\
\text { Fails to start }\end{array}$ & $\begin{array}{l}45 / 2.0 \mathrm{E}+3 \mathrm{~d} \\
3 / 6.4 \mathrm{E}+4 \mathrm{~h} \\
{[17]}\end{array}$ & & $\begin{array}{l}2.2 \mathrm{E}-2 / \mathrm{d}(3.8), \\
5.5 \mathrm{E}-5 / \mathrm{h}(10)\end{array}$ \\
\hline Fails to run & $\begin{array}{l}19 / 1.4 \mathrm{E}+5 \mathrm{~h} \\
{[18]}\end{array}$ & & $1.4 \mathrm{E}-4 / \mathrm{h}(22)$ \\
\hline \multicolumn{4}{|l|}{ Overspeed } \\
\hline \multicolumn{4}{|l|}{ Fails to stop } \\
\hline \multicolumn{4}{|l|}{ Leakage (external) } \\
\hline \multicolumn{4}{|l|}{ Rupture (external) } \\
\hline $\begin{array}{l}\text { Diesel-Driven } \\
\text { Fails to stan }\end{array}$ & $\begin{array}{l}15 / 1.9 \mathrm{E}+3 \mathrm{~d} \\
{[19]}\end{array}$ & & $7.9 \mathrm{E}-3 / \mathrm{d}(5.8)$ \\
\hline Fails to run & $\begin{array}{l}3 / 6.5 \mathrm{E}+2 \mathrm{~h} \\
{[20]}\end{array}$ & & $4.6 \mathrm{E}-3 / \mathrm{h}(7.4)$ \\
\hline \multicolumn{4}{|l|}{ Overspeed } \\
\hline \multicolumn{4}{|l|}{ Fails to stop } \\
\hline \multicolumn{4}{|l|}{ Leakage (external) } \\
\hline \multicolumn{4}{|l|}{ Ruphure (external) } \\
\hline \multicolumn{4}{|l|}{$\begin{array}{l}\text { Piping/Hose//umper } \\
\text { Piping } \\
\text { Leakage (external) }\end{array}$} \\
\hline Rupture (external) & & & \\
\hline Plugs & & & \\
\hline
\end{tabular}




\begin{tabular}{|c|c|c|c|}
\hline \multirow[t]{2}{*}{ System/Component/Failure Mode } & \multicolumn{3}{|c|}{ Failure Data (Category 1 Sources) } \\
\hline & NUCLARR1 & $\begin{array}{c}\text { SRS- } \\
\text { REACTORS }\end{array}$ & $\begin{array}{c}\text { Aggregated } \\
\text { Results }^{\mathrm{b}} \\
\end{array}$ \\
\hline \multicolumn{4}{|l|}{$\begin{array}{l}\text { Hose } \\
\quad \text { Leakage (external) }\end{array}$} \\
\hline \multicolumn{4}{|l|}{ Rupture (external) } \\
\hline \multicolumn{4}{|l|}{ Plugs } \\
\hline \multicolumn{4}{|l|}{$\begin{array}{l}\text { Jumper } \\
\text { Leakage (external) }\end{array}$} \\
\hline \multicolumn{4}{|l|}{ Rupture (external) } \\
\hline \multicolumn{4}{|l|}{ Plugs } \\
\hline $\begin{array}{l}\text { Vessel } \\
\text { Tank (Unpressurized) } \\
\text { Leakage (extemal) } \\
\end{array}$ & $\begin{array}{l}0 / 6.9 \mathrm{E}+5 \mathrm{~h} \\
{[21]}\end{array}$ & & $\begin{array}{l}7.3 \mathrm{E}-7 / \mathrm{h}(10) \\
{[1]}\end{array}$ \\
\hline Rupture (external) & [21] & & \\
\hline \multicolumn{4}{|l|}{$\begin{array}{l}\text { Tank (Pressurized) } \\
\text { Leakage (external) }\end{array}$} \\
\hline \multicolumn{4}{|l|}{ Rupture (external) } \\
\hline \multicolumn{4}{|l|}{$\begin{array}{l}\text { Flange/Gasket } \\
\text { Leakage (external) }\end{array}$} \\
\hline \multicolumn{4}{|l|}{ Rupture (external) } \\
\hline \multicolumn{4}{|l|}{$\begin{array}{l}\text { Heat Exchanger } \\
\text { Shell/Tube } \\
\text { Fouling (tubes) }\end{array}$} \\
\hline Plugs (tubes) & $\begin{array}{l}0 / 1.9 \mathrm{E}+6 \mathrm{~h} \\
{[22]}\end{array}$ & & $\begin{array}{l}2.6 \mathrm{E}-7 / \mathrm{h}(10) \\
{[1]}\end{array}$ \\
\hline Leakage (ubes) & $\begin{array}{l}0 / 3.8 \mathrm{E}+5 \mathrm{~h} \\
{[23]}\end{array}$ & & $\begin{array}{l}1.3 \mathrm{E}-6 / \mathrm{h}(10) \\
{[1]}\end{array}$ \\
\hline Rupture (tubes) & [23] & & \\
\hline Leakage (shell) & $\begin{array}{l}0 / 1.6 \mathrm{E}+6 \mathrm{~h} \\
{[24]}\end{array}$ & & $\begin{array}{l}3.1 \mathrm{E}-7 / \mathrm{h}(10) \\
{[1]}\end{array}$ \\
\hline Rupture (shell) & {$[24]$} & & \\
\hline \multicolumn{4}{|l|}{$\begin{array}{l}\text { Heater (Electrical) } \\
\text { Fails to heat } \\
\end{array}$} \\
\hline \multicolumn{4}{|l|}{ Overheats } \\
\hline Leakage (external) & & & \\
\hline
\end{tabular}




\begin{tabular}{||c|l|l|l||}
\hline Table 3a. Category 1 data for water system. \\
\hline System/Componen//Failure Mode & \multicolumn{3}{|c|}{ Failure Data (Category 1 Sources) } \\
\cline { 2 - 4 } & NUCLARR1 & \multicolumn{1}{|c|}{$\begin{array}{c}\text { SRS- } \\
\text { REACTORS }\end{array}$} & \multicolumn{1}{c|}{$\begin{array}{c}\text { Aggregated } \\
\text { Results }\end{array}$} \\
\hline \hline Rupture (external) & & & \\
\hline $\begin{array}{c}\text { Strainer/Filter } \\
\text { Plugs }\end{array}$ & $\begin{array}{l}2 / 6.0 \mathrm{E}+5 \mathrm{~h} \\
{[25]}\end{array}$ & $\begin{array}{l}0 / 2.5 \mathrm{E}+4 \mathrm{~h} \\
{[20]}\end{array}$ & $4.0 \mathrm{E}-6 / \mathrm{h}(10)$ \\
\hline Leakage (internal) & & & \\
\hline Rupture (internal) & & & \\
\hline $\begin{array}{c}\text { Orifice } \\
\text { Plugs }\end{array}$ & & & \\
\hline $\begin{array}{c}\text { Miscellaneous } \\
\text { Travelling Screen } \\
\text { Plugs }\end{array}$ & & $\begin{array}{l}0 / 9.6 \mathrm{E}+5 \mathrm{~h} \\
{[21]}\end{array}$ & $5.2 \mathrm{E}-7 / \mathrm{h}(10)$ \\
{$[1]$}
\end{tabular}

Table 3a. Category 1 data for water system.

a. Failure data are listed as: $\mathrm{X} / \mathrm{Y}$, where $\mathrm{X}$ is the number of failures, and $\mathrm{Y}$ is the exposure period (h) or number of demands. Numbers in brackets refer to notes listed below, arranged by source.

b. Aggregated results are mean and error factor (in parentheses). The error factor is the 95 th percentile/50th percentile.

Notes

Feactor PRAs, Idaho National Engineering Laboratory, EGG-SSRE-8875, February 1990.

All failure data were obtained from Tables 6 and 7 in the reference. These tables list the aggregations of Category 1 source data from NUCLARR as it existed in August 1989.

1. Manual valve fail to operate data used.

2. Check valve fail to open data used.

3. Check valve fail to close data used.

4. Check valve leakage data used. Size of leakage not indicated.

5. Motor-operated valve fail to operate data used.

6. Motor-operated valve spurious operation data used.

7. Pneumatic val \& fail to operate and fail to open daia used. 
8. Pneumatic valve spurious operation data used.

9. Pneumatic valve internal leakage data used. Size of leakage not indicated.

10. Solenoid-operated valve fail to operate data used.

11. Solenoid-operated valve spurious operation data used.

12. Relief valve fail to operate and fail to open data used.

13. Relief valve fail to close data used.

14. Vacuum-breaker valve fail to operate data used.

15. Motor-driven pump fail to start data used.

16. Motor-driven pump fail to run data used.

17. Turbine-driven pump fail to start data used.

Table 3a. Category 1 data for water system.

18. Turbine-driven pump fail to run data used.

19. Diesel-driven pump fail to start data used.

20. Diesel-driven pump fail to run data used.

21. Tank (unpressurized) external leakage data used. Size of leakage not indicated.

22. Heat exchanger tube plugged data used.

23. Heat exchanger internal leakage data used. Size of leakage not indicated.

24. Heat exchanger external leakage data used. Size of leakage not indicated.

25. Strainer plugged data used.

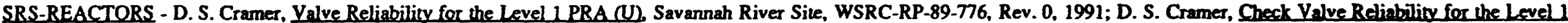

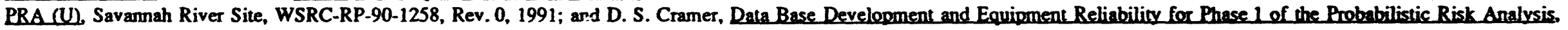
Savannah River Site, DPST-87-642, October 1987.

1. Data are from Table 27 (first reference, above) for gate and plug valves. Fail to open (CC) and fail to close (OO) data used.

2. Data are from Table 27 (first reference) for gate and plug valves. Fails to remain open (OC) data used. 
3. Data are from Table 27 (first reference) for gate and plug valves. Fails to remain closed (CO) data used. Size of internal leakage not indicated.

4. Data are from Table 1 (second reference). Failures are not broken down into fails to open and fails to close in this reference.

5. Data are from Table 1 (second reference). Fails to remain closed (CO) data used. Size of internal leakage not indicated.

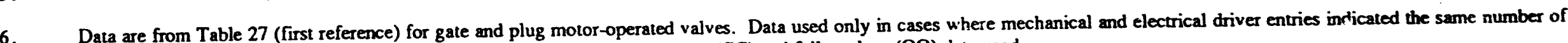
demands. Data include mechanical and electrical driver failures. Fail to open (CC) and fail to close (OO) data used.

7. See Note 6 above. Data include only electrical driver failures. Fails to remain closed (CO) and fails to remain open (OC) data used.

8. See Note 6 above. Data include only mechanical failures. Fails to remain open (OC) data used.

9. See Note 6 above. Data include only mechanical failures. Fails to remain closed (CO) data used. Size of internal leakage not indicated.

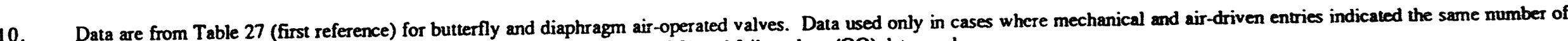
demands. Data include mechanical and air driver failures. Fail to open (CC) and fail to close (OO) data used.

11. See Note 10 above. Data include only air driver failures. Fails to remain closed (CO) and fails to remain open (OC) data used.

12. See Note 10 above. Data include only mechanical failures. Fails to remain open $(O C)$ data used.

13. Data are from Table 27 (first reference) for solenoid-operated valves. Fail to open (CC) and fail to close (OO) data used.

Table 3a. Category 1 data for water system.

14. See Note 13 above. Data include only solenoid driver failures. Fails to remain closed (CO) and fails to remain open (OC) data used.

15. See Note 13 above. Dara include only mechanical failures. Fails to remain open (OC) data used.

16. Data are from Table V.3 (third reference) for explosive valves. Fail to open (CC) data used.

17. Data are from Tables P.1, P.4, P.5, P.7, and P.8 (third reference). Data include mechanical and motor driver failures. Fail to start data used.

18. See Note 17 above. Fail to nun data used.

19. See Note 17 above. Fail to stop data used.

20. Data are from Table P.6 (third reference) for screens. Plugged failure data used.

21. Data are from Table W.1 (third reference) for travelling screen. Plugged failure data used. 
Aggregated Results

1. Failure rate based on zero failures, and may be conservatively high.

2. SRS-REACTOR data are not broken down into failure modes. NUCLARR1 data indicate that most failures are fail to close. Therefore, the SRS-REACTOR failure was assumed to be fails to close. 


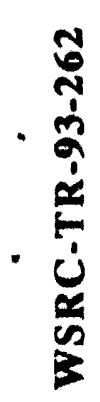

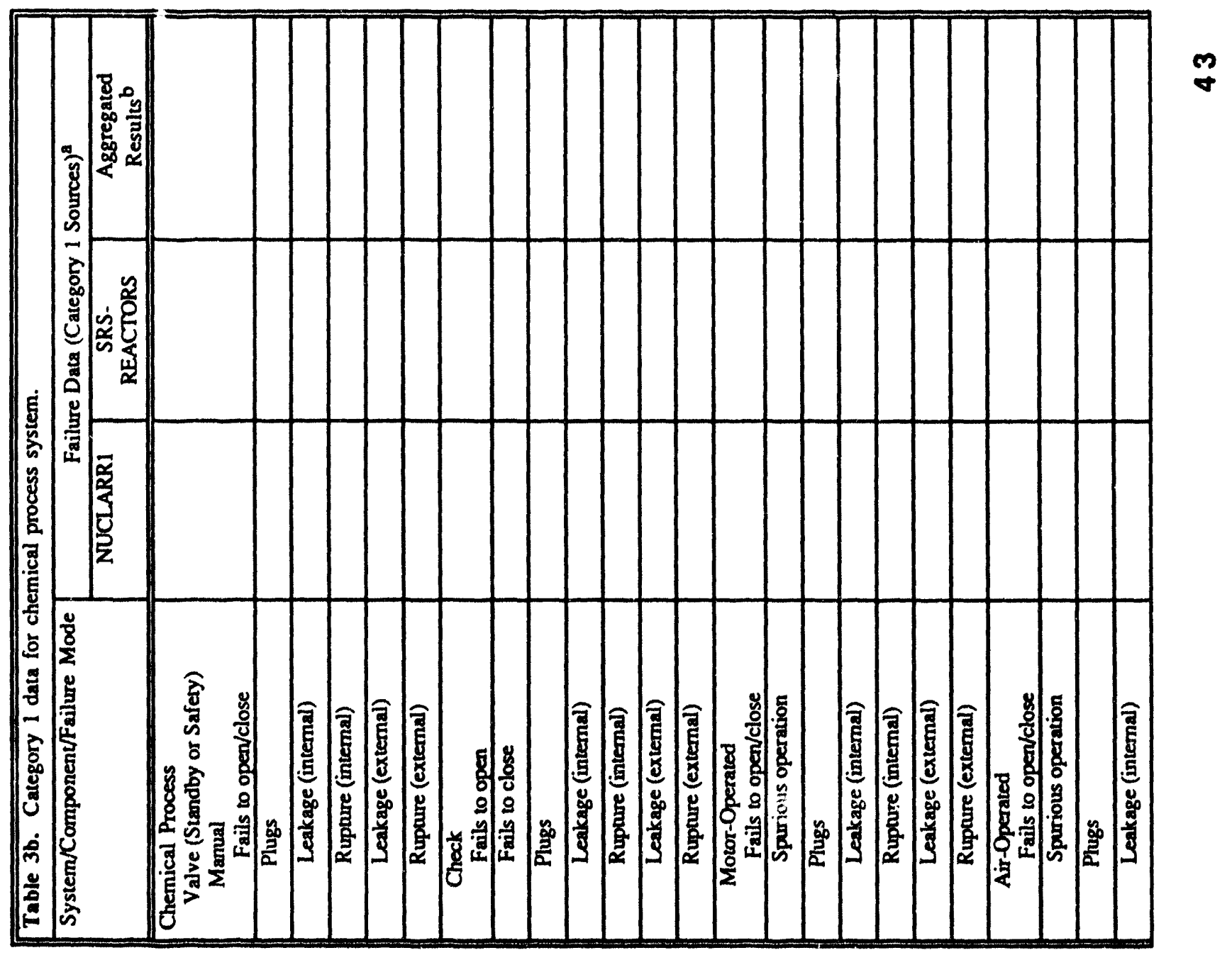




\begin{tabular}{|c|c|c|c|}
\hline \multirow[t]{2}{*}{ System/Componen/Failure Mode } & \multicolumn{3}{|c|}{ Failure Data (Category 1 Sources) } \\
\hline & NUCLARRI & $\begin{array}{c}\text { SRS- } \\
\text { REACTORS } \\
\end{array}$ & $\begin{array}{c}\text { Aggregated } \\
\text { Results }{ }^{b}\end{array}$ \\
\hline \multicolumn{4}{|l|}{ Rupture (internal) } \\
\hline \multicolumn{4}{|l|}{ Leakage (external) } \\
\hline \multicolumn{4}{|l|}{ Rupture (external) } \\
\hline \multicolumn{4}{|l|}{$\begin{array}{l}\text { Solenoid-Operated } \\
\text { Fails to open/close }\end{array}$} \\
\hline \multicolumn{4}{|l|}{ Spurious operation } \\
\hline \multicolumn{4}{|l|}{ Plugs } \\
\hline \multicolumn{4}{|l|}{ Leakage (internal) } \\
\hline \multicolumn{4}{|l|}{ Rupture (internal) } \\
\hline \multicolumn{4}{|l|}{ Leakage (external) } \\
\hline \multicolumn{4}{|l|}{ Rupture (external) } \\
\hline \multicolumn{4}{|l|}{$\begin{array}{l}\text { Safery/Relief } \\
\text { Fails to open }\end{array}$} \\
\hline \multicolumn{4}{|l|}{ Fails to reclose } \\
\hline \multicolumn{4}{|l|}{ Leakage (internal) } \\
\hline \multicolumn{4}{|l|}{ Rupture (internal) } \\
\hline \multicolumn{4}{|l|}{ Leakage (external) } \\
\hline \multicolumn{4}{|l|}{ Rupture (external) } \\
\hline \multicolumn{4}{|l|}{$\begin{array}{l}\text { Vacuum-Breaker } \\
\text { Fails to open }\end{array}$} \\
\hline \multicolumn{4}{|l|}{ Fails to reclose } \\
\hline \multicolumn{4}{|l|}{ Leakage (internal) } \\
\hline \multicolumn{4}{|l|}{ Rupture (internal) } \\
\hline \multicolumn{4}{|l|}{ Leakage (external) } \\
\hline \multicolumn{4}{|l|}{ Rupture (external) } \\
\hline \multicolumn{4}{|l|}{$\begin{array}{l}\text { Explosive } \\
\text { Fails to open }\end{array}$} \\
\hline Leakage (internal) & & & \\
\hline
\end{tabular}




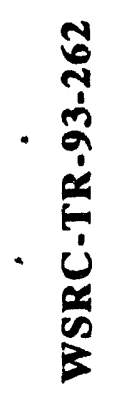

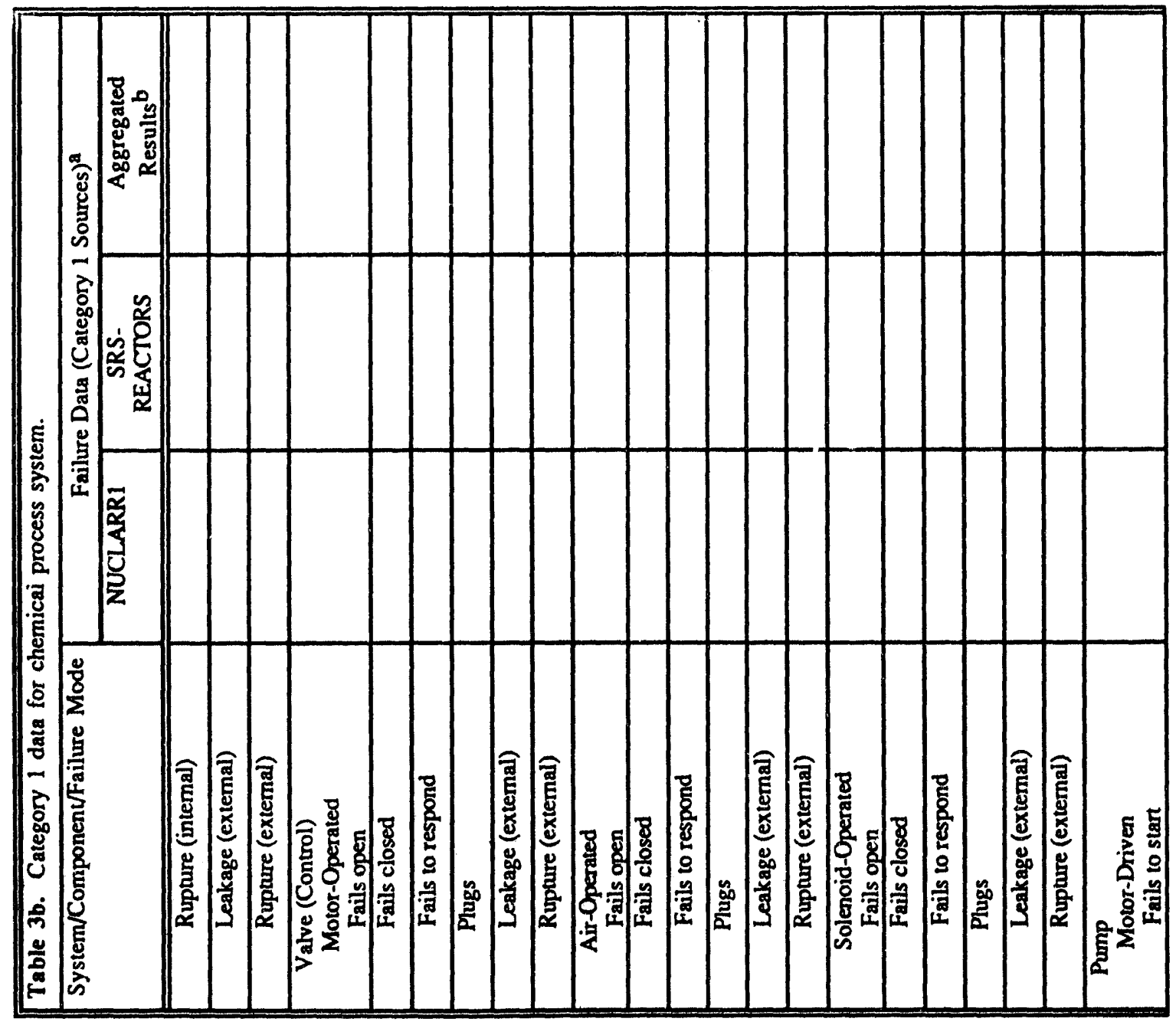

us 
WSRC-TR-93-262

Table 3b. Category 1 data for chemical process system.

\begin{tabular}{|l|l}
\hline System/Componen/Failure Mode & Failure Data (Category 1 Sources) \\
\end{tabular}

\begin{tabular}{|c|c|c|c||}
\hline \multirow{2}{*}{ System/Componen/Failure Mode } & \multicolumn{3}{|c|}{ Failure Data (Category 1 Sources) } \\
\hline & NUCLARR1 & $\begin{array}{c}\text { SRS- } \\
\text { REACTORS }\end{array}$ & $\begin{array}{c}\text { Aggregated } \\
\text { Results }\end{array}$ \\
\hline \hline Fails to run & & & \\
\hline Overspeed & & & \\
\hline Fails to stop & & & \\
\hline Leakage (external) & & & \\
\hline Rupture (external) & & & \\
\hline $\begin{array}{c}\text { Turbine-Driven } \\
\text { Fails to start }\end{array}$ & & & \\
\hline Fails to nun & & & \\
\hline Overspeed & & & \\
\hline Fails to stop & & & \\
\hline Leakage (external) & & & \\
\hline Rupture (extemal) & & & \\
\hline $\begin{array}{c}\text { Diesel-Driven } \\
\text { Fails to start }\end{array}$ & & & \\
\hline Fails to run & & & \\
\hline Overspeed & & & \\
\hline Fails to stop & & & \\
\hline Leakage (external) & & & \\
\hline Rupture (extemal) & & & \\
\hline $\begin{array}{c}\text { Piping/Hose/Jumper } \\
\text { Piping } \\
\text { Leakage (extemal) }\end{array}$ & & & \\
\hline Rupure (external) & & & \\
\hline Plugs & & & \\
\hline Hose \\
Leakage (external) & & & \\
\hline Rupture (extemal) & & & \\
\hline Plugs & & & \\
\hline
\end{tabular}




\begin{tabular}{|c|c|c|c|}
\hline \multirow[t]{2}{*}{ System/Component/Failure Mode } & \multicolumn{3}{|c|}{ Failure Data (Category 1 Sources) ${ }^{a}$} \\
\hline & NUCLARR1 & $\begin{array}{c}\text { SRS- } \\
\text { REACTORS }\end{array}$ & $\begin{array}{c}\text { Aggregated } \\
\text { Results }\end{array}$ \\
\hline \multicolumn{4}{|l|}{$\begin{array}{l}\text { Jumper } \\
\text { Leakage (external) }\end{array}$} \\
\hline \multicolumn{4}{|l|}{$\frac{\text { Leakage (extemal) }}{\text { Rupture (extermal) }}$} \\
\hline \multicolumn{4}{|l|}{$\begin{array}{l}\text { Plugs } \\
\end{array}$} \\
\hline \multicolumn{4}{|l|}{$\begin{array}{l}\text { Vessel } \\
\text { Tank (Unpressurized) } \\
\text { Leakage (extemal) }\end{array}$} \\
\hline \multicolumn{4}{|l|}{$\begin{array}{l}\text { Leakage (external) } \\
\text { Rupture (external) }\end{array}$} \\
\hline \multicolumn{4}{|l|}{$\begin{array}{l}\text { Tank (Pressurized) } \\
\text { Leakage (extemal) }\end{array}$} \\
\hline \multicolumn{4}{|l|}{ Rupture (external) } \\
\hline \multicolumn{4}{|l|}{$\begin{array}{l}\text { Flange/Gasket } \\
\text { Leakage (extemal) }\end{array}$} \\
\hline \multicolumn{4}{|l|}{$\begin{array}{l}\text { Leakage (external) } \\
\text { Rupture (external) }\end{array}$} \\
\hline \multicolumn{4}{|l|}{$\begin{array}{l}\text { Heat Exchanger } \\
\text { Shell/Tube } \\
\text { Fouling (tubes) }\end{array}$} \\
\hline \multicolumn{4}{|l|}{$\begin{array}{l}\text { Fouling (uubes) } \\
\text { Plugs (tubes) }\end{array}$} \\
\hline \multicolumn{4}{|l|}{ Leakage (tubes) } \\
\hline \multicolumn{4}{|l|}{ Rupture (ubes) } \\
\hline \multicolumn{4}{|l|}{ Leakage (shell) } \\
\hline \multicolumn{4}{|l|}{ Rupture (shell) } \\
\hline \multicolumn{4}{|l|}{$\begin{array}{l}\text { Heater (Electrical) } \\
\text { Fails to heat }\end{array}$} \\
\hline \multicolumn{4}{|l|}{$\begin{array}{l}\text { Faus to neal } \\
\text { Overheats }\end{array}$} \\
\hline \multicolumn{4}{|l|}{ Leakage (external) } \\
\hline Rupture (external) & & & \\
\hline $\begin{array}{l}\text { Strainer/Filter } \\
\text { Plugs }\end{array}$ & & & \\
\hline
\end{tabular}




\begin{tabular}{|c|c|c|c|}
\hline \multirow[t]{2}{*}{ System/Componen/Failure Mode } & \multicolumn{3}{|c|}{ Failure Data (Category 1 Sources) ${ }^{\mathrm{a}}$} \\
\hline & NUCLARR1 & $\begin{array}{c}\text { SRS- } \\
\text { REACTORS }\end{array}$ & $\begin{array}{c}\text { Aggregated } \\
\text { Results }{ }^{b}\end{array}$ \\
\hline Leakage (internal) & & & \\
\hline Rupture (internal) & & & \\
\hline $\begin{array}{l}\text { Orifice } \\
\text { Plugs }\end{array}$ & & & \\
\hline $\begin{array}{l}\text { Miscellaneous } \\
\text { Mixer/Blender } \\
\text { Failure }\end{array}$ & & & \\
\hline $\begin{array}{c}\text { Agitator } \\
\text { Failure }\end{array}$ & & & \\
\hline $\begin{array}{l}\text { Centrifuge } \\
\text { Failure }\end{array}$ & & & \\
\hline
\end{tabular}

Table 3b. Category 1 data for chemical process system.

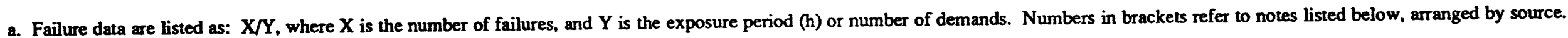

b. Aggregated results are mean and error factor (in parentheses). The error factor is the 95th percentile/50th percentile. 


\begin{tabular}{|c|c|c|c|}
\hline \multirow[t]{2}{*}{ System/Component/Failure Mode } & \multicolumn{3}{|c|}{ Failure Data (Category 1 Sources) ${ }^{a}$} \\
\hline & NUCLARR1 & $\begin{array}{c}\text { SRS- } \\
\text { REACTORS } \\
\end{array}$ & $\begin{array}{c}\text { Aggregated } \\
\text { Results } \\
\end{array}$ \\
\hline \multicolumn{4}{|l|}{$\begin{array}{l}\text { Compressed Gas } \\
\text { Valve (Standby or Safety) } \\
\text { Manual } \\
\text { Fails to open/close } \\
\end{array}$} \\
\hline \multicolumn{4}{|l|}{ Plugs } \\
\hline \multicolumn{4}{|l|}{ Leakage (internal) } \\
\hline \multicolumn{4}{|l|}{ Rupture (internal) } \\
\hline \multicolumn{4}{|l|}{ Leakage (external) } \\
\hline \multicolumn{4}{|l|}{ Rupture (external) } \\
\hline \multicolumn{4}{|l|}{$\begin{array}{l}\text { Check } \\
\text { Fails to open }\end{array}$} \\
\hline \multicolumn{4}{|l|}{$\begin{array}{l}\text { Fails to open } \\
\text { Fails to close }\end{array}$} \\
\hline \multicolumn{4}{|l|}{ Plugs } \\
\hline \multicolumn{4}{|l|}{ Leakage (intemal) } \\
\hline \multicolumn{4}{|l|}{ Rupture (internal) } \\
\hline \multicolumn{4}{|l|}{ Leakage (external) } \\
\hline \multicolumn{4}{|l|}{ Rupture (external) } \\
\hline \multicolumn{4}{|l|}{$\begin{array}{l}\text { Motor-Operated } \\
\text { Fails to open/close }\end{array}$} \\
\hline \multicolumn{4}{|l|}{$\begin{array}{l}\text { Fails to open/close } \\
\text { Spurious operation }\end{array}$} \\
\hline \multicolumn{4}{|l|}{ Plugs } \\
\hline \multicolumn{4}{|l|}{ Leakage (intemal) } \\
\hline \multicolumn{4}{|l|}{ Rupture (internal) } \\
\hline \multicolumn{4}{|l|}{ Leakage (extemal) } \\
\hline \multicolumn{4}{|l|}{ Rupture (external) } \\
\hline \multicolumn{4}{|l|}{$\begin{array}{l}\text { Air-Operated } \\
\text { Fails to open/close }\end{array}$} \\
\hline \multicolumn{4}{|l|}{ Spurious operation } \\
\hline \multicolumn{4}{|l|}{ Plugs } \\
\hline Leakage (internal) & & & \\
\hline
\end{tabular}


WSRC-TR-93-262

\begin{tabular}{|c|c|c|c|}
\hline \multirow[t]{2}{*}{ System/Component/Failure Mode } & \multicolumn{3}{|c|}{ Failure Data (Category 1 Sources) $)^{\mathrm{a}}$} \\
\hline & NUCLARR1 & $\begin{array}{c}\text { SRS- } \\
\text { REACTORS }\end{array}$ & $\begin{array}{l}\text { Aggregated } \\
\text { Results }^{\mathrm{b}}\end{array}$ \\
\hline \multicolumn{4}{|l|}{ Rupture (internal) } \\
\hline \multicolumn{4}{|l|}{ Leakage (extemal) } \\
\hline \multicolumn{4}{|l|}{ Rupture (external) } \\
\hline \multicolumn{4}{|l|}{$\begin{array}{l}\text { Solenoid-Operated } \\
\text { Fails to open/close }\end{array}$} \\
\hline \multicolumn{4}{|l|}{ Spurious operation } \\
\hline \multicolumn{4}{|l|}{ Plugs } \\
\hline \multicolumn{4}{|l|}{ Leakage (internal) } \\
\hline \multicolumn{4}{|l|}{ Rupture (intemal) } \\
\hline \multicolumn{4}{|l|}{ Leakage (external) } \\
\hline \multicolumn{4}{|l|}{ Rupture (external) } \\
\hline \multicolumn{4}{|l|}{$\begin{array}{l}\text { Safety/Relief } \\
\text { Fails to open } \\
\end{array}$} \\
\hline \multicolumn{4}{|l|}{ Fails to reclose } \\
\hline \multicolumn{4}{|l|}{ Leakage (internal) } \\
\hline \multicolumn{4}{|l|}{ Rupture (internal) } \\
\hline \multicolumn{4}{|l|}{ Leakage (external) } \\
\hline \multicolumn{4}{|l|}{ Rupture (extemal) } \\
\hline \multicolumn{4}{|l|}{$\begin{array}{l}\text { Vacuum-Breaker } \\
\text { Fails to open }\end{array}$} \\
\hline \multicolumn{4}{|l|}{ Fails to reclose } \\
\hline \multicolumn{4}{|l|}{ Leakage (internal) } \\
\hline \multicolumn{4}{|l|}{ Rupture (internal) } \\
\hline \multicolumn{4}{|l|}{ Leakage (external) } \\
\hline \multicolumn{4}{|l|}{ Rupture (external) } \\
\hline $\begin{array}{l}\text { Valve (Control) } \\
\text { Motor-Operated } \\
\text { Fails open }\end{array}$ & & & \\
\hline Fails closed & & & \\
\hline
\end{tabular}




\begin{tabular}{|c|c|c|c|}
\hline \multirow[t]{2}{*}{ System/Component/Failure Mode } & \multicolumn{3}{|c|}{ Failure Data (Category 1 Sources) ${ }^{\mathrm{a}}$} \\
\hline & NUCLARR1 & $\begin{array}{c}\text { SRS- } \\
\text { REACTORS }\end{array}$ & $\begin{array}{c}\text { Aggregated } \\
\text { Results }^{\mathrm{b}}\end{array}$ \\
\hline \multicolumn{4}{|l|}{ Fails to respond } \\
\hline \multicolumn{4}{|l|}{ Plugs } \\
\hline \multicolumn{4}{|l|}{ Leakage (extemal) } \\
\hline \multicolumn{4}{|l|}{ Rupture (external) } \\
\hline \multicolumn{4}{|l|}{$\begin{array}{l}\text { Air-Operated } \\
\text { Fails open }\end{array}$} \\
\hline \multicolumn{4}{|l|}{ Fails closed } \\
\hline \multicolumn{4}{|l|}{ Fails to respond } \\
\hline \multicolumn{4}{|l|}{ Plugs } \\
\hline \multicolumn{4}{|l|}{ Leakage (external) } \\
\hline \multicolumn{4}{|l|}{ Rupture (external) } \\
\hline \multicolumn{4}{|l|}{$\begin{array}{l}\text { Solenoid-Operated } \\
\text { Fails open }\end{array}$} \\
\hline \multicolumn{4}{|l|}{$\begin{array}{l}\text { Fauls open } \\
\text { Fails closed }\end{array}$} \\
\hline \multicolumn{4}{|l|}{ Fails to respond } \\
\hline \multicolumn{4}{|l|}{ Plugs } \\
\hline \multicolumn{4}{|l|}{ Leakage (external) } \\
\hline \multicolumn{4}{|l|}{ Rupture (external) } \\
\hline $\begin{array}{l}\text { Compressor } \\
\text { Motor-Driven } \\
\text { Fails to start } \\
\end{array}$ & $\begin{array}{l}10 / 1.4 \mathrm{E}+3 \mathrm{~d} \\
{[1]}\end{array}$ & $\begin{array}{l}2 / 3.3 \mathrm{E}+2 \mathrm{~d}, \\
4 / 4.4 \mathrm{E}+2 \mathrm{~d} \\
{[1]}\end{array}$ & $7.5 \mathrm{E}-3 / \mathrm{d}(7.1)$ \\
\hline Fails to run & $\begin{array}{l}23 / 3.0 \mathrm{E}+5 \mathrm{~h} \\
{[2]}\end{array}$ & $\begin{array}{l}6 / 1.7 E+5 h, \\
2 / 8.9 E+4 h \\
{[2]}\end{array}$ & $5.6 \mathrm{E}-5 / \mathrm{h}(1.2)$ \\
\hline \multicolumn{4}{|l|}{ Overspeed } \\
\hline \multicolumn{4}{|l|}{ Fails to ston } \\
\hline \multicolumn{4}{|l|}{ Leakage (external) } \\
\hline Rupture (external) & & & \\
\hline
\end{tabular}


部

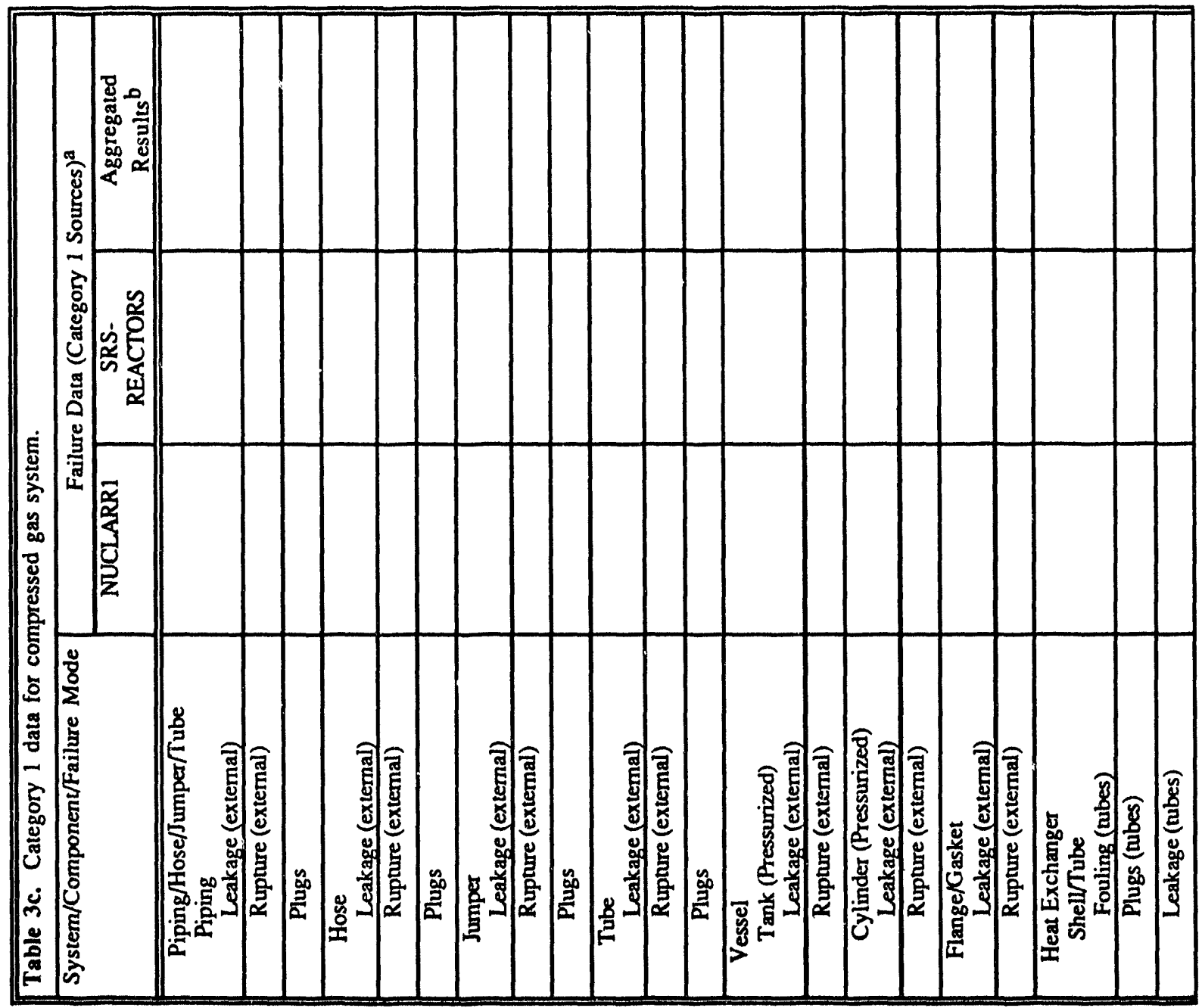

in 


\begin{tabular}{|c|c|c|c|}
\hline \multirow[t]{2}{*}{ System/Component/Failure Mode } & \multicolumn{3}{|c|}{ Failure Data (Category 1 Sources) ${ }^{\mathrm{a}}$} \\
\hline & NUCLARR1 & $\begin{array}{c}\text { SRS- } \\
\text { REACTORS } \\
\end{array}$ & $\begin{array}{c}\text { Aggregated } \\
\text { Results } \\
\text { b }\end{array}$ \\
\hline \multicolumn{4}{|l|}{ Rupture (tubes) } \\
\hline \multicolumn{4}{|l|}{ Leakage (shell) } \\
\hline \multicolumn{4}{|l|}{ Rupture (shell) } \\
\hline \multicolumn{4}{|l|}{$\begin{array}{l}\text { Heater (Electrical) } \\
\text { Fails to heat }\end{array}$} \\
\hline \multicolumn{4}{|l|}{ Overheats } \\
\hline \multicolumn{4}{|l|}{ Leakage (external) } \\
\hline \multicolumn{4}{|l|}{ Rupture (external) } \\
\hline \multicolumn{4}{|l|}{ 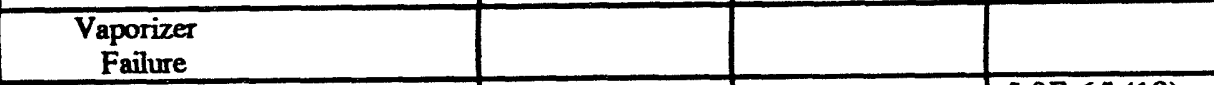 } \\
\hline $\begin{array}{c}\text { Air Dryer } \\
\text { Failure } \\
\end{array}$ & $\begin{array}{l}0 / 8.6 \mathrm{E}+4 \mathrm{~h} \\
{[3]}\end{array}$ & & $\begin{array}{l}5.8 \mathrm{E}-6 / \mathrm{h}(10) \\
{[1]}\end{array}$ \\
\hline \multicolumn{4}{|l|}{$\begin{array}{l}\text { Filter } \\
\quad \text { Plugs } \\
\end{array}$} \\
\hline \multicolumn{4}{|l|}{ Leakage (internal) } \\
\hline Rupture (intemal) & & & \\
\hline $\begin{array}{c}\text { Orifice } \\
\text { Plugs }\end{array}$ & & & \\
\hline
\end{tabular}

Table 3c. Category 1 data for compressed gas system.

a. Failure data are listed as: $\mathrm{X} / \mathrm{Y}$, where $\mathrm{X}$ is the number of failures, and $\mathrm{Y}$ is the exposure period $(\mathrm{h})$ or number of demands. Numbers in brackets refer to notes listed below, arranged by source.

b. Aggregated results are mean and error factor (in parentheses). The error factor is the 95 th percentile/50th percentile.

Notes

NUCLARR1 - S. A. Eide et al.. Generic Component Failure Data Base for Light Water and Liquid Sodium Reactor PRAs, Idaho National Engineering Laboratory, EGG-SSRE-8875, February 1990.

All failure data were obtained from Tables 6 and 7 in the reference. These tables list the aggregations of Category 1 source data from NUCLARR as it existed in August 1989. 
1. Blower/compressor fail to start data used.

2. Blower/compressor fail to run data used.

3. Air dryer fail to operate data used.

SRS-REACTORS - D. S. Cramer, Data Base Development and Equipment Reliability for Phase 1 of the Probabilistic Risk Analysis, Savannah River Site, DPST-87-642, October 1987.

1. Data are from Tables G.2 and G.3. Air dryer failures are included in the data. Fail to start data used.

2. Data are from Tables G.2 and G.3. Air dryer failures are included in the data. Fail to run data used.

\section{Aggregated Results}

1. Failure rate based on zero failures and may be conservatively high. 


\begin{tabular}{|c|c|c|c|}
\hline \multirow[t]{2}{*}{ System/Component/Failure Mode } & \multicolumn{3}{|c|}{ Failure Data (Calegory 1 Sources) ${ }^{a}$} \\
\hline & NUCLARR1 & $\begin{array}{c}\text { SRS- } \\
\text { REACTORS } \\
\end{array}$ & $\begin{array}{c}\text { Aggregated } \\
\text { Results }{ }^{b}\end{array}$ \\
\hline \multicolumn{4}{|l|}{$\begin{array}{l}\text { HVAC/Exhaust } \\
\text { Damper (Standby or Safety) } \\
\text { Manual } \\
\text { Fails wo open/close }\end{array}$} \\
\hline \multicolumn{4}{|l|}{ Plugs } \\
\hline \multicolumn{4}{|l|}{ Leakage (internal) } \\
\hline \multicolumn{4}{|l|}{ Rupture (internal) } \\
\hline \multicolumn{4}{|l|}{ Leakage (external) } \\
\hline \multicolumn{4}{|l|}{ Rupture (external) } \\
\hline \multicolumn{4}{|l|}{$\begin{array}{l}\text { Motor-Operated } \\
\text { Fails to open/close }\end{array}$} \\
\hline \multicolumn{4}{|l|}{ Spurious operation } \\
\hline \multicolumn{4}{|l|}{ Plugs } \\
\hline \multicolumn{4}{|l|}{ Leskage (intemal) } \\
\hline \multicolumn{4}{|l|}{ Rupture (internal) } \\
\hline \multicolumn{4}{|l|}{ Leakage (external) } \\
\hline \multicolumn{4}{|l|}{ Rupture (extemal) } \\
\hline $\begin{array}{l}\text { Air-Operated } \\
\text { Fails to open/close }\end{array}$ & $\begin{array}{l}3 / 1.2 E+3 d \\
{[1]}\end{array}$ & $\begin{array}{l}10 / 3.5 \mathrm{E}+2 \mathrm{~d}, \\
17 / 2.1 \mathrm{E}+2 \mathrm{~d} \\
12 / 2.5 \mathrm{E}+3 \mathrm{~d}, \\
8 / 5.3 \mathrm{E}+2 \mathrm{~d}, \\
2 / 5.3 \mathrm{E}+2 \mathrm{~d} \\
{[1]}\end{array}$ & $9.9 \mathrm{E}-3 / \mathrm{d}(6.1)$ \\
\hline Spurious operation & & $\begin{array}{l}5 / 2.9 \mathrm{E}+5 \mathrm{~h}, \\
5 / 9.2 \mathrm{E}+5 \mathrm{~h}, \\
0 / 8.7 \mathrm{E}+5 \mathrm{~h}, \\
10 / 2.9 \mathrm{E}+5 \mathrm{~h}, \\
3 / 2.9 \mathrm{E}+5 \mathrm{~h}, \\
1 / 2.9 \mathrm{E}+5 \mathrm{~h} \\
{[2]}\end{array}$ & $8.2 \mathrm{E}-6 / \mathrm{h}(4.2)$ \\
\hline \multicolumn{4}{|l|}{ Plugs } \\
\hline \multicolumn{4}{|l|}{ Leakage (internal) } \\
\hline Rupture (internal) & & & \\
\hline
\end{tabular}


WSRC-TR-93-262

\begin{tabular}{|c|c|c|c|}
\hline \multirow[t]{2}{*}{ System/Component/Failure Mode } & \multicolumn{3}{|c|}{ Failure Data (Category 1 Sources) ${ }^{\mathrm{a}}$} \\
\hline & NUCLARR1 & $\begin{array}{c}\text { SRS- } \\
\text { REACTORS }\end{array}$ & $\begin{array}{c}\text { Aggregated } \\
\text { Results }\end{array}$ \\
\hline \multicolumn{4}{|l|}{ Leakage (external) } \\
\hline \multicolumn{4}{|l|}{ Ruphure (external) } \\
\hline \multicolumn{4}{|l|}{$\begin{array}{l}\text { Damper (Control) } \\
\text { Motor-Operated } \\
\text { Fails open }\end{array}$} \\
\hline \multicolumn{4}{|l|}{ Fails closed } \\
\hline \multicolumn{4}{|l|}{ Fails to respond } \\
\hline \multicolumn{4}{|l|}{ Plugs } \\
\hline \multicolumn{4}{|l|}{ Leakage (external) } \\
\hline \multicolumn{4}{|l|}{ Rupture (extemal) } \\
\hline \multicolumn{4}{|l|}{$\begin{array}{l}\text { Air-Operated } \\
\text { Fails open }\end{array}$} \\
\hline \multicolumn{4}{|l|}{ Fails closed } \\
\hline \multicolumn{4}{|l|}{ Fails to respond } \\
\hline \multicolumn{4}{|l|}{ Plugs } \\
\hline \multicolumn{4}{|l|}{ Leakage (external) } \\
\hline \multicolumn{4}{|l|}{ Rupture (external) } \\
\hline $\begin{array}{l}\text { Fan/Blower } \\
\text { Motor-Driven } \\
\text { Fails to start }\end{array}$ & $\begin{array}{l}11 / 1.5 \mathrm{E}+3 \mathrm{~d} \\
{[2]}\end{array}$ & $\begin{array}{l}1 / 1.2 \mathrm{E}+3 \mathrm{~d} \\
{[3]}\end{array}$ & $4.4 \mathrm{E}-3 / \mathrm{d}(5.4)$ \\
\hline Fails to run & $\begin{array}{l}13 / 5.2 \mathrm{E}+5 \mathrm{~h} \\
{[3]}\end{array}$ & $\begin{array}{l}14 / 4.2 \mathrm{E}+5 \mathrm{~h} \\
{[4]}\end{array}$ & $2.9 \mathrm{E}-5 / \mathrm{h}(2.4)$ \\
\hline \multicolumn{4}{|l|}{ Overspeed } \\
\hline \multicolumn{4}{|l|}{ Fails to stop } \\
\hline \multicolumn{4}{|l|}{ Leakage (external) } \\
\hline \multicolumn{4}{|l|}{ Rupture (extemal) } \\
\hline \multicolumn{4}{|l|}{$\begin{array}{l}\text { Diesel-Driven } \\
\text { Fails to start }\end{array}$} \\
\hline Fails to run & & & \\
\hline Overspeed & & & \\
\hline
\end{tabular}

56 
Table 3d. Category 1 data for HVAC/exhaust system.

\begin{tabular}{|c|c|c|c|}
\hline \multirow[t]{2}{*}{ System/Component/Failure Mode } & \multicolumn{3}{|c|}{ Failure Data (Category 1 Sources) ${ }^{a}$} \\
\hline & NUCLARR1 & $\begin{array}{c}\text { SRS- } \\
\text { REACTORS }\end{array}$ & $\begin{array}{l}\text { Aggregated } \\
\text { Results }{ }^{b}\end{array}$ \\
\hline \multicolumn{4}{|l|}{ Fails to stop } \\
\hline \multicolumn{4}{|l|}{ Leakage (external) } \\
\hline \multicolumn{4}{|l|}{ Rupture (external) } \\
\hline \multicolumn{4}{|l|}{$\begin{array}{l}\text { Ducting } \\
\text { Leakage (external) }\end{array}$} \\
\hline \multicolumn{4}{|l|}{ Rupture (extemal) } \\
\hline \multicolumn{4}{|l|}{ Phugs } \\
\hline $\begin{array}{l}\text { Heat Exchanger } \\
\text { Air Conditioning Univ } \\
\text { Chiller } \\
\text { Fails to start }\end{array}$ & $\begin{array}{l}8 / 6.1 E+2 d \\
{[4]}\end{array}$ & & $1.4 \mathrm{E}-2 / \mathrm{d}(10)$ \\
\hline Fails to run & $\begin{array}{l}4 / 1.9 \mathrm{E}+5 \mathrm{~h} \\
{[5]}\end{array}$ & & $2.4 \mathrm{E}-5 / \mathrm{h}(10)$ \\
\hline $\begin{array}{l}\text { Fan Cooler Unit } \\
\text { Fails to start }\end{array}$ & $\begin{array}{l}21 / 2.5 E+3 d \\
{[6]}\end{array}$ & & $8.4 \mathrm{E}-3 / \mathrm{d}(7.2)$ \\
\hline Fails to run & $\begin{array}{l}2 / 2.3 \mathrm{E}+5 \mathrm{~h} \\
{[7]}\end{array}$ & & $8.8 E-6 / h(2.9)$ \\
\hline \multicolumn{4}{|l|}{$\begin{array}{l}\text { Heater (Electrical) } \\
\text { Filis in heat }\end{array}$} \\
\hline \multicolumn{4}{|l|}{ Guthas } \\
\hline \multicolumn{4}{|l|}{$\begin{array}{l}\text { Heater (Gas) } \\
\text { Fails to heat }\end{array}$} \\
\hline \multicolumn{4}{|l|}{ Overheats } \\
\hline \multicolumn{4}{|l|}{$\begin{array}{l}\text { Filter } \\
\text { Normal } \\
\text { Plugs } \\
\end{array}$} \\
\hline \multicolumn{4}{|l|}{ Leakage (internal) } \\
\hline \multicolumn{4}{|l|}{ Rupture (internal) } \\
\hline \multicolumn{4}{|l|}{$\begin{array}{l}\text { Low-Efficiency } \\
\text { Phugs }\end{array}$} \\
\hline \multicolumn{4}{|l|}{ Leskage (intemal) } \\
\hline Rupture (internal) & & & \\
\hline
\end{tabular}




\begin{tabular}{|c|c|c|c|}
\hline \multirow[t]{2}{*}{ System/Component/Failure Mode } & \multicolumn{3}{|c|}{ Failure Data (Category 1 Sources) ${ }^{\mathrm{a}}$} \\
\hline & NUCLARR1 & $\begin{array}{c}\text { SRS- } \\
\text { REACTORS } \\
\end{array}$ & $\begin{array}{c}\text { Aggregated } \\
\text { Results }^{\mathrm{b}} \\
\end{array}$ \\
\hline \multicolumn{4}{|l|}{$\begin{array}{l}\text { HEPA } \\
\text { Plugs }\end{array}$} \\
\hline \multicolumn{4}{|l|}{ Leakage (internal) } \\
\hline \multicolumn{4}{|l|}{ Rupaure (internal) } \\
\hline \multicolumn{4}{|l|}{$\begin{array}{l}\text { Sand } \\
\text { Plugs }\end{array}$} \\
\hline \multicolumn{4}{|l|}{$\begin{array}{l}\text { Pugs } \\
\text { Leakage (internal) }\end{array}$} \\
\hline \multicolumn{4}{|l|}{ Rupture (internal) } \\
\hline \multicolumn{4}{|l|}{$\begin{array}{l}\text { Baghouse } \\
\text { Phugs }\end{array}$} \\
\hline \multicolumn{4}{|l|}{ Prugs } \\
\hline \multicolumn{4}{|l|}{ Rupture (internal) } \\
\hline \multicolumn{4}{|l|}{$\begin{array}{l}\text { Miscellaneous } \\
\text { Mist Eliminator } \\
\text { Failure }\end{array}$} \\
\hline \multicolumn{4}{|l|}{$\begin{array}{l}\text { Scrubber } \\
\text { Failure }\end{array}$} \\
\hline $\begin{array}{l}\text { Precipitator } \\
\text { Failure }\end{array}$ & & & \\
\hline
\end{tabular}

Table 3d. Category 1 data for HVAC/exhaust system.

a. Fsilure data are listed as: $\mathrm{X} / \mathrm{Y}$, where $\mathrm{X}$ is the number of failures, and $\mathrm{Y}$ is the exposure period (h) or number of demands. Numbers in brackets refer wo notes listed below, arranged by source.

b. Aggregated results are mean and error factor (in parentheses). The error factor is the 95th percentile/50th percencile.

\section{Notes}

NUCLARR1 - S. A. Eide et al, Generic Compenent Failure Data Base for Light Water and Liquid Sodium Reactor PRAs, Idaho National Engineering Laboratory, EGG-SSRE-8875, February 1990.

All faihure data were obtained from Tables 6 and 7 in the reference. These tables list the aggregations of Category 1 source data from NUCLARR as it existed in August 1989. 
1. Damper fail to operate data used. Type of damper not indicated (air-operated assumed).

2. Ventilator fan fail to start data used.

3. Ventilator tail to run data used.

4. Air conditioning unit fail to start data used.

5. Air conditioning unit fail to run data used.

6. Fan/cooler unit fail to start data used.

7. Fan/cooler unit fail to run data used.

SRS-REACTORS - D. S. Cramer, Data Base Development and Equipment Reliability for Phase 1 of the Probabilistic Risk Analysis, Savannah River

S. Cramer, Reliability of Dampers in the Reactor Confinement System, Savannah River Site, SRL-PRA-900011, Revision 3, January 25, 1990.

1. Data are from Table 1 (second reference). Fail to open (CC) and fail to close (OO) data used.

2. Data are fren Table 1 (second reference). Fails to remain open $(O C)$ and fails to remain closed (CO) data used.

3. Data are from Tables VENT.7, VENT.8, and VENT.9 (first reference). Fails to start data used.

4. Data are from Tables VENT.7, VENT.8, and VENT.9 (first reference). Fails to run data used. 


\begin{tabular}{|c|c|c|c|}
\hline \multirow[t]{2}{*}{ System/Componen/Failure Mode } & \multicolumn{3}{|c|}{ Failure Data (Category 1 Sources) } \\
\hline & NUCLARR1 & $\begin{array}{c}\text { SRS- } \\
\text { REACTORS } \\
\end{array}$ & $\begin{array}{c}\text { Aggregated } \\
\text { Results } \\
\end{array}$ \\
\hline $\begin{array}{l}\text { Electric Power } \\
\text { Generator } \\
\text { Diesel-Driven } \\
\text { Fails to start } \\
\end{array}$ & $\begin{array}{l}92 / 8.7 E+3 d \\
{[1]}\end{array}$ & $\begin{array}{l}26 / 9.1 \mathrm{E}+2 \mathrm{~d} \\
16 / 9.5 \mathrm{E}+2 \mathrm{~d} \\
{[1]}\end{array}$ & $1.3 \mathrm{E}-2 / \mathrm{d}(2.2)$ \\
\hline Fails to run & $\begin{array}{l}29 / 6.6 \mathrm{E}+3 \mathrm{~h} \\
{[2]}\end{array}$ & $\begin{array}{l}1 / 5.8 \mathrm{E}+2 \mathrm{~h} \\
{[2]}\end{array}$ & $4.1 \mathrm{E}-3 / \mathrm{h}(3.3)$ \\
\hline \multicolumn{4}{|l|}{$\begin{array}{l}\text { Motor-Driven (ac to dc) } \\
\text { Fails to start }\end{array}$} \\
\hline Fails to run & $\begin{array}{l}31 / 1.5 \mathrm{E}+6 \mathrm{~h} \\
{[3]}\end{array}$ & & $2.1 \mathrm{E}-5 / \mathrm{h}(10)$ \\
\hline $\begin{array}{l}\text { Gas-Turbine-Driven } \\
\text { Fails to start } \\
\end{array}$ & $\begin{array}{l}35 / 9.4 \mathrm{E}+2 \mathrm{~d} \\
{[4]}\end{array}$ & & $3.8 \mathrm{E}-2 / \mathrm{d}(10)$ \\
\hline Fails to run & $\begin{array}{l}1 / 5.7 \mathrm{E}+3 \mathrm{~h} \\
{[5]}\end{array}$ & & $2.6 \mathrm{E}-4 / \mathrm{h}(10)$ \\
\hline $\begin{array}{l}\text { Hydro-Turbine-Driven } \\
\text { Fails to start } \\
\end{array}$ & $\begin{array}{l}12 / 4.1 \mathrm{E}+3 \mathrm{~d} \\
{[6]}\end{array}$ & & $3.1 \mathrm{E}-3 / \mathrm{d}(10)$ \\
\hline \multicolumn{4}{|l|}{ Fails to run } \\
\hline $\begin{array}{l}\text { Battery } \\
\text { Failure }\end{array}$ & $\begin{array}{l}7 / 5.9 \mathrm{E}+2 \mathrm{~d} \\
8 / 9.4 \mathrm{E}+5 \mathrm{~h} \\
{[7]}\end{array}$ & $\begin{array}{l}12 / 1.8 \mathrm{E}+3 \mathrm{~d} \\
{[3]}\end{array}$ & $\begin{array}{l}8.0 \mathrm{E}-3 / \mathrm{d}(1.2) \\
8.4 \mathrm{E}-6 / \mathrm{h}(3.7)\end{array}$ \\
\hline $\begin{array}{l}\text { Charger } \\
\text { Rectifier } \\
\text { Failure } \\
\end{array}$ & $\begin{array}{l}29 / 1.6 \mathrm{E}+6 \mathrm{~h} \\
{[8]}\end{array}$ & & $1.8 \mathrm{E}-5 / \mathrm{h}(1.7)$ \\
\hline $\begin{array}{l}\text { Bus } \\
\text { Metal-Enclosed } \\
\text { Failure } \\
\end{array}$ & $\begin{array}{l}1 / 1.4 \mathrm{E}+7 \mathrm{~h} \\
{[9]}\end{array}$ & & $1.1 \mathrm{E}-7 / \mathrm{h}(10)$ \\
\hline $\begin{array}{l}\text { Bare } \\
\text { Failure } \\
\end{array}$ & [9] & & \\
\hline \multicolumn{4}{|l|}{$\begin{array}{l}\text { Failure } \\
\text { Cable/Joint/ } \\
\text { Termination/Jumper } \\
\text { Cable (Copper, 1000ft) } \\
\text { Failure }\end{array}$} \\
\hline \multicolumn{4}{|l|}{$\begin{array}{l}\text { Joint (Copper) } \\
\text { Failure } \\
\end{array}$} \\
\hline $\begin{array}{l}\text { Termination (Copper) } \\
\text { Failure }\end{array}$ & & & \\
\hline
\end{tabular}


Table 3e. Category 1 data for electrical distribution system.

\begin{tabular}{|c|c|c|c|}
\hline \multirow[t]{2}{*}{ System/Component/Failure Mode } & \multicolumn{3}{|c|}{ Failure Data (Category 1 Sources) ${ }^{\mathrm{a}}$} \\
\hline & NUCLARR1 & $\begin{array}{c}\text { SRS- } \\
\text { REACTORS } \\
\end{array}$ & $\begin{array}{c}\text { Aggregated } \\
\text { Results }^{\mathrm{b}} \\
\end{array}$ \\
\hline \multicolumn{4}{|l|}{$\begin{array}{l}\text { Jumper (Power) } \\
\text { Failure }\end{array}$} \\
\hline $\begin{array}{l}\text { Circuit Breaker } \\
\text { General } \\
\text { Fails to open/close }\end{array}$ & $\begin{array}{l}101 / 1.8 E+5 d \\
{[10]}\end{array}$ & $\begin{array}{l}4 / 1.3 E+3 d \\
{[4]}\end{array}$ & $5.6 \mathrm{E}-4 / \mathrm{d}(4.5)$ \\
\hline Spurious operation & $\begin{array}{l}15 / 4.1 \mathrm{E}+7 \mathrm{~h} \\
{[111}\end{array}$ & & $3.8 \mathrm{E}-7 / \mathrm{h}(10)$ \\
\hline \multicolumn{4}{|l|}{$\begin{array}{l}\text { Reactor Trip } \\
\text { Fails to open } \\
\end{array}$} \\
\hline \multicolumn{4}{|l|}{$\begin{array}{l}\text { Fails to open } \\
\text { Spurious operation }\end{array}$} \\
\hline 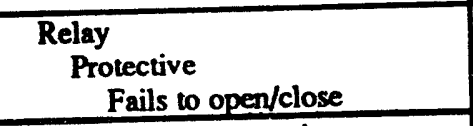 & & $\begin{array}{l}5 / 4.5 E+3 d \\
{[5]}\end{array}$ & $1.2 \mathrm{E}-3 / \mathrm{d}(10)$ \\
\hline \multicolumn{4}{|l|}{$\begin{array}{l}\text { Fails to open/close } \\
\text { Spurious operation }\end{array}$} \\
\hline \multicolumn{4}{|l|}{$\begin{array}{l}\text { Control } \\
\text { Fails to open/close }\end{array}$} \\
\hline \multicolumn{4}{|l|}{$\begin{array}{l}\text { Falis to open/ciose } \\
\text { Spurious operation }\end{array}$} \\
\hline \multicolumn{4}{|l|}{$\begin{array}{l}\text { Bistable } \\
\text { Fails to open/close }\end{array}$} \\
\hline \multicolumn{4}{|l|}{$\begin{array}{l}\text { Fails to open/ciose } \\
\text { Spurious operation }\end{array}$} \\
\hline \multicolumn{4}{|l|}{$\begin{array}{l}\text { Switch } \\
\text { Push-Button (Manual) } \\
\text { Fails to open/close }\end{array}$} \\
\hline \multicolumn{4}{|l|}{$\begin{array}{l}\text { Fails to open/ciose } \\
\text { Spurious operation }\end{array}$} \\
\hline \multicolumn{4}{|l|}{$\begin{array}{l}\text { Rotary (Manual) } \\
\text { Fails to open/close }\end{array}$} \\
\hline \multicolumn{4}{|l|}{$\begin{array}{l}\text { Fails to open/close } \\
\text { Spurious operation }\end{array}$} \\
\hline \multicolumn{4}{|l|}{ Key-Operated (Manual) } \\
\hline \multicolumn{4}{|l|}{ Fails to open/close } \\
\hline Spurious operation & & & \\
\hline $\begin{array}{l}\text { Automatic-Transfer } \\
\text { Fails to open/close }\end{array}$ & $\begin{array}{l}1 / 7.5 \mathrm{E}+1 \mathrm{~d} \\
{[12]}\end{array}$ & & $2.0 \mathrm{E}-2 / \mathrm{d}(10)$ \\
\hline
\end{tabular}


WSRC-TR-93-262

\begin{tabular}{|c|c|c|c|}
\hline \multirow[t]{2}{*}{ System/Component/Failure Mode } & \multicolumn{3}{|c|}{ Failure Data (Category 1 Sources) ${ }^{2}$} \\
\hline & NUCLARR1 & $\begin{array}{c}\text { SRS- } \\
\text { REACTORS }\end{array}$ & $\begin{array}{c}\text { Aggregated } \\
\text { Results }\end{array}$ \\
\hline \multicolumn{4}{|l|}{ Spurious operation } \\
\hline $\begin{array}{l}\text { Limit } \\
\text { Fails to open/close }\end{array}$ & $\begin{array}{l}0 / 1.3 \mathrm{E}+4 \mathrm{~d} \\
7 / 8.2 \mathrm{E}+6 \mathrm{~h} \\
{[13]}\end{array}$ & & $\begin{array}{l}4.0 \mathrm{E}-5 / \mathrm{d}(10) \\
9.2 \mathrm{E}-7 / \mathrm{h}(10) \\
{[1]}\end{array}$ \\
\hline \multicolumn{4}{|l|}{ Spurious operation } \\
\hline \multicolumn{4}{|l|}{$\begin{array}{l}\text { Fuse } \\
\text { Fail to open }\end{array}$} \\
\hline \multicolumn{4}{|l|}{ Premature opening } \\
\hline $\begin{array}{l}\text { Inverter } \\
\text { Failure }\end{array}$ & $\begin{array}{l}0 / 6.3 \mathrm{E}+1 \mathrm{~d} \\
23 / 1.9 \mathrm{E}+6 \mathrm{~h} \\
{[14]}\end{array}$ & & $\begin{array}{l}7.9 \mathrm{E}-3 / \mathrm{d}(10) \\
1.2 \mathrm{E}-5 / \mathrm{h}(1.9) \\
{[1]}\end{array}$ \\
\hline \multicolumn{4}{|l|}{$\begin{array}{l}\text { Motor } \\
\text { AC } \\
\text { Fails to start }\end{array}$} \\
\hline \multicolumn{4}{|l|}{ Fails to run } \\
\hline \multicolumn{4}{|l|}{$\begin{array}{l}\text { DC } \\
\text { Fails to start }\end{array}$} \\
\hline \multicolumn{4}{|l|}{ Fails to run } \\
\hline \multicolumn{4}{|l|}{$\begin{array}{l}\text { Synchro } \\
\text { Failure }\end{array}$} \\
\hline $\begin{array}{l}\text { Transformer } \\
\text { Power } \\
\text { Failure } \\
\end{array}$ & $\begin{array}{l}1 / 1.8 \mathrm{E}+6 \mathrm{~h} \\
{[15]}\end{array}$ & & $8.2 \mathrm{E}-7 / \mathrm{h}(10)$ \\
\hline $\begin{array}{l}\text { Instrumentation/Control } \\
\text { Failure }\end{array}$ & $\begin{array}{l}5 / 6.2 \mathrm{E}+6 \\
{[16]}\end{array}$ & & $8.9 \mathrm{E}-7 / \mathrm{h}(10)$ \\
\hline
\end{tabular}

Table 3e. Category 1 data for electrical distribution system.

a. Failure data are listed as: $\mathrm{X} / \mathrm{Y}$, where $\mathrm{X}$ is the number of failures, and $\mathrm{Y}$ is the exposure period $(\mathrm{h})$ or number of demands. Numbers in brackets refer to notes listed below, arranged by source.

b. Aggregated results are mean and error factor (in parentheses). The error factor is the 95 th percentile/ 50 th percentile. 
Notes

NUCLARR1 - S. A. Eide et al., Generic Component Failure Data Base for Light Water and Liquid Sodium Reactor PRAs, Idaho National Engineering Laboratory, EGG-SSRE-8875, February 1990.

All failure data were obtained from Tables 6 and 7 in the reference. These tables list the aggregations of Category 1 source data from NUCLARR as it existed in August 1989.

1. Diesel generator fail to start data used.

2. Diesel generator fail to run data used.

3. Motor-driven generator fai! fo run data used.

4. Gas-turbine-driven generator fail to start data used.

5. Gas-turbine-driven generator fail to run data used.

6. Hydro-turbine-driven generator fail to start data used.

7. Battery fail to operate data used.

8. Battery charger fail to operate data used.

9. Electrical conductor (bus) fail to operate data used. Type of bus not indicated.

10. Circuit breaker data (except for spurious failure mode) used.

11. Circuit breaker spurious data used.

12. Automatic transfer switch fail to operate data used.

13. Instrumentation (limit) switch fail to operate data used.

14. Power electronics (inverter) fail to operate data used.

15. Power transformer fail to operate data used.

16. Control and instrumentation transformer fail to operate data used.

Table 3e. Category 1 data for electrical distribution system. 
SRS-REACTORS - D. S. Cramer, Data Base Development and Equipment Reliability for Phase 1 of the Probabilistic Risk Analysis, Savannah River Site, DPST-87-642, October 1987.

1. Data are from Tables E.10 and VENT.5. Fail to start data used.

2. Data are from Tables E.10 and VENT.5. Fail to run data used.

3. Data are from Table E.1.

4. Data are from 'Table E.11.

5. Data are from Table E.13.

\section{Aggregated Results}

1. Failure rate based on zero failures and may be conservatively high. 


\begin{tabular}{|c|c|c|c|}
\hline \multirow[t]{2}{*}{ System/Component/Failure Mode } & \multicolumn{3}{|c|}{ Failure Data (Category 1 Sources) $)^{a}$} \\
\hline & NUCLARR1 & $\begin{array}{c}\text { SRS- } \\
\text { REACTORS } \\
\end{array}$ & $\begin{array}{c}\text { Aggregated } \\
\text { Results } \mathrm{b}\end{array}$ \\
\hline \multicolumn{4}{|l|}{$\begin{array}{l}\text { Instrumentation and Control } \\
\text { Alarm/Annunciator } \\
\text { Fails to alarm } \\
\end{array}$} \\
\hline \multicolumn{4}{|l|}{ Spurious operation } \\
\hline $\begin{array}{l}\text { Sensor/Transmitter/ } \\
\text { Transducer/Process Switch } \\
\text { Temperature } \\
\text { Failure }\end{array}$ & $\begin{array}{l}12 / 5.7 \mathrm{E}+4 \mathrm{~d} \\
78 / 7.6 \mathrm{E}+7 \mathrm{~h} \\
{[1]}\end{array}$ & $\begin{array}{l}2 / 8.7 \mathrm{E}+5 \mathrm{~h} \\
6 / 1.2 \mathrm{E}+6 \mathrm{~h} \\
{[1]}\end{array}$ & $\begin{array}{l}2.8 \mathrm{E}-4 / \mathrm{d}(3.5) \\
1.1 \mathrm{E}-6 / \mathrm{h}(3.3)\end{array}$ \\
\hline $\begin{array}{l}\text { Pressure } \\
\text { Failure }\end{array}$ & [1] & & $\begin{array}{l}2.8 \mathrm{E}-4 / \mathrm{d}(3.5) \\
1.1 \mathrm{E}-6 / \mathrm{h}(3.3)\end{array}$ \\
\hline \multicolumn{4}{|l|}{$\begin{array}{l}\text { Differential Pressure } \\
\text { Failure }\end{array}$} \\
\hline \multicolumn{4}{|l|}{$\begin{array}{l}\text { Flow } \\
\text { Failure }\end{array}$} \\
\hline \multicolumn{4}{|l|}{$\begin{array}{l}\text { Level } \\
\text { Failure }\end{array}$} \\
\hline \multicolumn{4}{|l|}{$\begin{array}{l}\text { Humidity } \\
\text { Failure }\end{array}$} \\
\hline \multicolumn{4}{|l|}{ FH } \\
\hline \multicolumn{4}{|l|}{$\begin{array}{l}\text { Oxygen Concentration } \\
\text { Failure }\end{array}$} \\
\hline \multicolumn{4}{|l|}{$\begin{array}{l}\mathrm{CO}_{2} \text { Concentration } \\
\text { Failure }\end{array}$} \\
\hline \multicolumn{4}{|l|}{$\begin{array}{l}\text { Hydrogen Concentration } \\
\text { Failure }\end{array}$} \\
\hline \multicolumn{4}{|l|}{$\begin{array}{l}\text { Nitrogen Concentration } \\
\text { Failure }\end{array}$} \\
\hline \multicolumn{4}{|l|}{$\begin{array}{l}\text { Hydrocarbon Concentration } \\
\text { Failure }\end{array}$} \\
\hline \multicolumn{4}{|l|}{$\begin{array}{l}\text { Helium Concentration } \\
\text { Failure }\end{array}$} \\
\hline \multicolumn{4}{|l|}{$\begin{array}{l}\text { Speed } \\
\text { Failure } \\
\end{array}$} \\
\hline $\begin{array}{c}\text { Seismic } \\
\text { Failure }\end{array}$ & & & \\
\hline
\end{tabular}




\begin{tabular}{|c|c|c|c|}
\hline \multirow[t]{2}{*}{ System/Component/Failure Mode } & \multicolumn{3}{|c|}{ Failure Data (Category 1 Sources) ${ }^{\mathrm{a}}$} \\
\hline & NUCLARR1 & $\begin{array}{c}\text { SRS- } \\
\text { REACTORS }\end{array}$ & $\begin{array}{c}\text { Aggregated } \\
\text { Results }\end{array}$ \\
\hline $\begin{array}{c}\text { Raciation } \\
\text { Failure } \\
\end{array}$ & & & \\
\hline $\begin{array}{c}\text { Indicator } \\
\text { Failure } \\
\end{array}$ & & & \\
\hline $\begin{array}{l}\text { Amplifier } \\
\text { Failure }\end{array}$ & & & \\
\hline $\begin{array}{l}\text { Modifier/Signal Conditioner } \\
\text { Failure }\end{array}$ & & & \\
\hline $\begin{array}{l}\text { Logic Module } \\
\text { Failure }\end{array}$ & & & \\
\hline $\begin{array}{c}\text { Recorder } \\
\text { Failure }\end{array}$ & & & \\
\hline $\begin{array}{c}\text { Sampler } \\
\text { Failure }\end{array}$ & & & \\
\hline $\begin{array}{c}\text { An:lyzer } \\
\text { Failure } \\
\end{array}$ & & & \\
\hline $\begin{array}{l}\text { Timer } \\
\text { Failure } \\
\end{array}$ & & & \\
\hline $\begin{array}{l}\text { Gas Chromatograph } \\
\text { Failure }\end{array}$ & & & \\
\hline $\begin{array}{l}\text { Voltage Regulator } \\
\text { Failure }\end{array}$ & & & \\
\hline $\begin{array}{c}\text { Transmitter } \\
\text { Failure } \\
\end{array}$ & $\begin{array}{l}57 / 2.6 \mathrm{E}+7 \mathrm{~h} \\
{[2]}\end{array}$ & & $2.2 \mathrm{E}-6 / \mathrm{h}(1.4)$ \\
\hline $\begin{array}{l}\text { Transducer } \\
\text { Failure }\end{array}$ & & & \\
\hline $\begin{array}{l}\text { Programmable Logic Controller } \\
\text { Failure }\end{array}$ & & & \\
\hline
\end{tabular}

Table 3r. Category 1 data for instrumentation and control system.

a Failure data are listed as: $\mathrm{X} / \mathrm{Y}$, where $\mathrm{X}$ is the number of failures, and $\mathrm{Y}$ is the exposure period (h) or number of demands. Numbers in brackets refer to notes listed below, arranged by source. b. Aggregated results are mean and error factor (in parentheses). The error factor is the 95 th percentile/50th percentile. Notes 
作 1990. All failure data were obtaines from Tables 6 and 7 in the reference. These tables list the aggregations of Category 1 source data from NUCLARR as it existed in August 1989.

1. Transducer (sensor) data used. First data entry is for failure to operate. Second data entry is for spurious operation. Type of sensor not indicated.

2. Transmitter failure to operate data used.

Data Base Develooment and Equipment Reliability for Phase 1 of the Probabilistic Risk. Analysis, Savannah River Site, DPST-87-642, October 1987.

1. Thermocouple data from Table E.16 used. 
WSRC-TR-93-262

\begin{tabular}{|c|c|c|c|c|c|c|c|}
\hline \multirow[t]{2}{*}{ System/Component/Failure Mode } & \multicolumn{7}{|c|}{ Failure Data (Category 2 Sources) $)^{\mathrm{a}}$} \\
\hline & NUCLARR2 & NPRD-3 & OREDA & WIN-330 & TRIIUM & LNG1 & $\begin{array}{c}\text { Aggregated } \\
\text { Results } \\
\end{array}$ \\
\hline $\begin{array}{l}\text { Water } \\
\text { Valve (Standby or Safety) } \\
\text { Manual } \\
\text { Fails to open/close }\end{array}$ & $\begin{array}{l}9 / 6.5 E+4 d \\
{[1]}\end{array}$ & $\begin{array}{l}2.5 / 3.8 \mathrm{E}+6 \mathrm{~h}, \\
1.5 / 2.5 \mathrm{E}+6 \mathrm{~h} \\
{[1]}\end{array}$ & & & & & $\begin{array}{l}1.4 \mathrm{E}-4 / \mathrm{d}(19) \\
7.1 \mathrm{E}-7 / \mathrm{h}(10)\end{array}$ \\
\hline \multicolumn{8}{|l|}{ Plugs } \\
\hline \multicolumn{8}{|l|}{ Leakage (internal) } \\
\hline \multicolumn{8}{|l|}{ Rupture (internal) } \\
\hline Leakage (external) & $\begin{array}{l}124 / 1.3 \mathrm{E}+10 \mathrm{~h} \\
{[2]}\end{array}$ & $\begin{array}{l}5 / 3.8 \mathrm{E}+6 \mathrm{~h}, \\
3 / 2.5 \mathrm{E}+6 \mathrm{~h} \\
{[1]}\end{array}$ & & & & & $1.0 \mathrm{E}-8 / \mathrm{h}(9.6)$ \\
\hline Rupture (external) & $\begin{array}{l}7 / 1.3 \mathrm{E}+10 \mathrm{~h} \\
{[3]}\end{array}$ & $\begin{array}{l}0 / 3.8 \mathrm{E}+6 \mathrm{~h}, \\
0 / 2.5 \mathrm{E}+6 \mathrm{~h}\end{array}$ & & & & & $5.8 \mathrm{E}-10 / \mathrm{h}(10)$ \\
\hline $\begin{array}{l}\text { Check } \\
\text { Fails to open }\end{array}$ & $\begin{array}{l}3 / 4.7 E+4 d \\
{[4]}\end{array}$ & $\begin{array}{l}3.3 / 2.2 \mathrm{E}+7 \mathrm{~h}, \\
1.4 / 1.8 \mathrm{E}+7 \mathrm{~h} \\
{[2]}\end{array}$ & & & & & $\begin{array}{l}1.8 \mathrm{E}-5 / \mathrm{d}(10) \\
1.3 \mathrm{E}-7 / \mathrm{h}(10)\end{array}$ \\
\hline Fails to close & [4] & 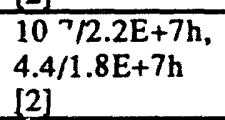 & & & & & $\begin{array}{l}\text { 5.7E-5/d(10), } \\
3.9 \mathrm{E}-7 / \mathrm{h}(10)\end{array}$ \\
\hline \multicolumn{8}{|l|}{ Plugs } \\
\hline Leakage (internal) & $\begin{array}{l}55 / 1.0 \mathrm{E}+8 \mathrm{~h} \\
{[5]}\end{array}$ & $\begin{array}{l}52.9 / 2.2 \mathrm{E}+7 \mathrm{~h}, \\
21.8 / 1.8 \mathrm{E}+7 \mathrm{~h} \\
{[3]}\end{array}$ & & & & & $9.1 \mathrm{E}-7 / \mathrm{h}(2.9)$ \\
\hline Rupture (internal) & [5] & [3] & & & & & \\
\hline Leakage (external) & $\begin{array}{l}124 / 1.3 \mathrm{E}+10 \mathrm{~h} \\
{[2]}\end{array}$ & $\begin{array}{l}52.9 / 2.2 \mathrm{E}+7 \mathrm{~h}, \\
21.8 / 1.8 \mathrm{E}+7 \mathrm{~h} \\
{[3]}\end{array}$ & & & & & $1.5 \mathrm{E}-8 / \mathrm{h}(26)$ \\
\hline Rupture (external) & $\begin{array}{l}7 / 1.3 \mathrm{E}+10 \mathrm{~h} \\
{[3]}\end{array}$ & $\begin{array}{l}0 / 2.2 \mathrm{E}+7 \mathrm{~h} \\
0 / 1.8 \mathrm{E}+7 \mathrm{~h} \\
{[4]}\end{array}$ & & & & & $5.8 \mathrm{E}-10 / \mathrm{h}(10)$ \\
\hline $\begin{array}{l}\text { Motor-Operated } \\
\text { Fails to open/close }\end{array}$ & 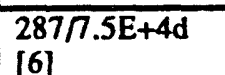 & & & & & & $3.8 \mathrm{E}-3 / \mathrm{d}(1.8)$ \\
\hline Spurious operation & $\begin{array}{l}24 / 1.4 \mathrm{E}+8 \mathrm{~h} \\
{[7]}\end{array}$ & & & & & & $1.8 \mathrm{E}-7 / \mathrm{h}(11)$ \\
\hline
\end{tabular}


WSRC-TR-93-262

\begin{tabular}{|c|c|c|c|c|c|c|c|}
\hline \multirow[t]{2}{*}{ System/Component/Failure Mode } & \multicolumn{7}{|c|}{ Failure Data (Category 2 Sources) ${ }^{\mathbf{a}}$} \\
\hline & NUCLARR2 & NPRD-3 & OREDA & WIN-330 & TRITIUM & LNG1 & $\begin{array}{c}\text { Aggregated } \\
\text { Results }^{\mathrm{b}}\end{array}$ \\
\hline \multicolumn{8}{|l|}{ Plugs } \\
\hline \multicolumn{8}{|l|}{ Leakage (internal) } \\
\hline \multicolumn{8}{|l|}{ Rupture (internal) } \\
\hline Leakage (external) & $\begin{array}{l}124 / 1.3 \mathrm{E}+10 \mathrm{~h} \\
{[2]}\end{array}$ & & & & & & $9.6 \mathrm{E}-9 / \mathrm{h}(10)$ \\
\hline Ruphure (external) & $7 / 1.3 \mathrm{E}+10 \mathrm{~h}$ & & & & & & $5.8 \mathrm{E}-10 / \mathrm{h}(10)$ \\
\hline $\begin{array}{l}\text { Air-Operated } \\
\text { Faiis to open/close }\end{array}$ & $\begin{array}{l}85 / 2.9 \mathrm{E}+4 \mathrm{~d} \\
{[8]}\end{array}$ & & $\begin{array}{l}16 / 8.9 \mathrm{E}+2 \mathrm{~d} \\
{[1]}\end{array}$ & & & & $3.4 \mathrm{E}-3 / \mathrm{d}(3.8)$ \\
\hline Spurious operation & $\begin{array}{l}10 / 1.1 \mathrm{E}+7 \mathrm{~h} \\
{[9]}\end{array}$ & & $\begin{array}{l}9 / 4.3 E+6 h \\
{[1]}\end{array}$ & & & & $1.2 \mathrm{E}-6 / \mathrm{h}(2.0)$ \\
\hline \multicolumn{8}{|l|}{ Plugs } \\
\hline Leakage (internal) & $\begin{array}{l}8 / 1.4 \mathrm{E}+7 \mathrm{~h} \\
{[10]}\end{array}$ & & $\begin{array}{l}7 / 4.3 \mathrm{E}+6 \mathrm{~h} \\
{[1]}\end{array}$ & & & & $6.9 \mathrm{E}-7 / \mathrm{h}(2.6)$ \\
\hline Rupture (intemal) & {$[10]$} & & $\begin{array}{l}3 / 4.3 \mathrm{E}+6 \mathrm{~h} \\
{[1]}\end{array}$ & & & & $3.0 \mathrm{E}-7 / \mathrm{h}(2.6)$ \\
\hline Leakage (external) & $\begin{array}{l}124 / 1.3 \mathrm{E}+10 \mathrm{~h} \\
{[2]}\end{array}$ & & $\begin{array}{l}5 / 4.3 \mathrm{E}+6 \mathrm{~h} \\
{[1]}\end{array}$ & & & & $9.9 \mathrm{E}-9 / \mathrm{h}(7.6)$ \\
\hline Rupture (external) & $\begin{array}{l}7 / 1.3 \mathrm{E}+10 \mathrm{~h} \\
{[3]}\end{array}$ & & $\begin{array}{l}0 / 4.3 \mathrm{E}+6 \mathrm{~h} \\
{[1]}\end{array}$ & & & & $5.8 \mathrm{E}-10 / \mathrm{h}(10)$ \\
\hline $\begin{array}{l}\text { Solenoid-Operated } \\
\text { Fails to open/close }\end{array}$ & $\begin{array}{l}96 / 9.0 \mathrm{E}+3 \mathrm{~d} \\
{[11]}\end{array}$ & $\begin{array}{l}0.3 / 4.6 \mathrm{E}+4 \mathrm{~h}, \\
2.8 / 4.8 \mathrm{E}+7 \mathrm{~h} \\
{[5]}\end{array}$ & & & & & $\begin{array}{l}\text { 1.1E-2/d(10), } \\
7.5 \mathrm{E}-8 / \mathrm{h}(10)\end{array}$ \\
\hline Spurious operation & $\begin{array}{l}1 / 8.8 \mathrm{E}+4 \mathrm{~h} \\
{[12]}\end{array}$ & $\begin{array}{l}0.1 / 4.6 \mathrm{E}+4 \mathrm{~h}, \\
1.2 / 4.8 \mathrm{E}+7 \mathrm{~h} \\
{[5]}\end{array}$ & & & & & $5.6 \mathrm{E}-8 / \mathrm{h}(10)$ \\
\hline \multicolumn{8}{|l|}{ Plugs } \\
\hline \multicolumn{8}{|l|}{ Leakage (internal) } \\
\hline \multicolumn{8}{|l|}{ Ruphure (internal) } \\
\hline Leakage (external) & $\begin{array}{l}124 / 1.3 \mathrm{E}+10 \mathrm{~h} \\
{[2]}\end{array}$ & $\begin{array}{l}3.9 / 4.6 \mathrm{E}+4 \mathrm{~h} \\
41.4 / 4.8 \mathrm{E}+7 \mathrm{~h} \\
{[5]}\end{array}$ & & & & & $1.3 \mathrm{E}-8 / \mathrm{h}(37)$ \\
\hline
\end{tabular}


WSRC-TR-93-262

\begin{tabular}{|c|c|c|c|c|c|c|c|}
\hline \multirow[t]{2}{*}{ System/Component/Failure Mode } & \multicolumn{7}{|c|}{ Failure Data (Category 2 Sources) ${ }^{\mathrm{a}}$} \\
\hline & NUCLARR2 & NPRD-3 & OREDA & WIN-330 & TRITUM & LNG1 & $\begin{array}{c}\text { Aggregated } \\
\text { Results } \\
\end{array}$ \\
\hline Rupture (external) & $\begin{array}{l}7 / 1.3 \mathrm{E}+10 \mathrm{~h} \\
{[3]}\end{array}$ & $\begin{array}{l}0 / 4.6 \mathrm{E}+4 \mathrm{~h}, \\
0 / 4.8 \mathrm{E}+7 \mathrm{~h} \\
{[5]}\end{array}$ & & & & & $5.8 \mathrm{E}-10 / \mathrm{h}(10)$ \\
\hline $\begin{array}{l}\text { Safety/Relief } \\
\text { Fails to open }\end{array}$ & $\begin{array}{l}130 / 4.9 E+4 d \\
{[13]}\end{array}$ & & & & & & $1.2 \mathrm{E}-2 / \mathrm{d}(3.3)$ \\
\hline \multicolumn{8}{|l|}{ Fails to reclose } \\
\hline Leakage (internal) & & $\begin{array}{l}4 / 9.7 \mathrm{E}+5 \mathrm{~h}, \\
7.5 / 9.2 \mathrm{E}+6 \mathrm{~h} \\
{[6]}\end{array}$ & & & & & $1.1 \mathrm{E}-6 / \mathrm{h}(2.5)$ \\
\hline \multicolumn{8}{|l|}{ Rupture (internal) } \\
\hline Leakage (external) & & $\begin{array}{l}4 / 9.7 \mathrm{E}+5 \mathrm{~h}, \\
7.5 / 9.2 \mathrm{E}+6 \mathrm{~h} \\
{[6]}\end{array}$ & & & & & $1.1 \mathrm{E}-6 / \mathrm{h}(2.5)$ \\
\hline Rupture (external) & & $\begin{array}{l}0 / 9.7 \mathrm{E}+5 \mathrm{~h}, \\
0 / 9.2 \mathrm{E}+6 \mathrm{~h} \\
{[6]}\end{array}$ & & & & & $4.9 \mathrm{E}-8 / \mathrm{h}(10)$ \\
\hline \multicolumn{8}{|l|}{$\begin{array}{c}\text { Vacinum-Breaker } \\
\text { Fails to open }\end{array}$} \\
\hline \multicolumn{8}{|l|}{ Fails to reclose } \\
\hline \multicolumn{8}{|l|}{ Leakage (internal) } \\
\hline \multicolumn{8}{|l|}{ Rupture (intemal) } \\
\hline \multicolumn{8}{|l|}{ Leakage (external) } \\
\hline \multicolumn{8}{|l|}{ Rupture (external) } \\
\hline \multicolumn{8}{|l|}{$\begin{array}{l}\text { Valve (Centrol) } \\
\text { Motor-Operated } \\
\text { Fails open } \\
\end{array}$} \\
\hline \multicolumn{8}{|l|}{ Fails closed } \\
\hline \multicolumn{8}{|l|}{ Fails to respond } \\
\hline \multicolumn{8}{|l|}{ Phugs } \\
\hline \multicolumn{8}{|l|}{ Leakage (externai) } \\
\hline Rupture (external) & & & & & & & \\
\hline
\end{tabular}


Table 4a. Category 2 data for water sy:tem (cont'd).

\begin{tabular}{|c|c|c|c|c|c|c|c|}
\hline \multirow[t]{2}{*}{ System/Component/Failure Mode } & \multicolumn{7}{|c|}{ Failure Data (Category 2 Sources) } \\
\hline & TUCLARR2 & NPRD-3 & OREDA & WIN-330 & TRITIUM & LNG1 & $\begin{array}{l}\text { Aggregaled } \\
\text { Results }\end{array}$ \\
\hline \multicolumn{8}{|l|}{$\begin{array}{l}\text { Air-Operated } \\
\text { Fails open }\end{array}$} \\
\hline \multicolumn{8}{|l|}{ Fails closed } \\
\hline \multicolumn{8}{|l|}{ Fails to respond } \\
\hline \multicolumn{8}{|l|}{ Phugs } \\
\hline \multicolumn{8}{|l|}{ Leakage (external) } \\
\hline \multicolumn{8}{|l|}{ Rupture (external) } \\
\hline \multicolumn{8}{|l|}{$\begin{array}{l}\text { Solenoid-Operated } \\
\text { Fails open }\end{array}$} \\
\hline \multicolumn{8}{|l|}{ Fails closed } \\
\hline \multicolumn{8}{|l|}{ Fails to respond } \\
\hline \multicolumn{8}{|l|}{ Phugs } \\
\hline \multicolumn{8}{|l|}{ Leakage (external) } \\
\hline \multicolumn{8}{|l|}{ Rupture (external) } \\
\hline $\begin{array}{l}\text { Pump } \\
\text { Moto-Driven } \\
\text { Fails to start }\end{array}$ & $\begin{array}{l}84 / 1.0 \mathrm{E}+5 \mathrm{~d} \\
{[14]}\end{array}$ & $\begin{array}{l}1.4 / 8.4 \mathrm{E}+5 \mathrm{~h}, \\
2.9 / 2.6 \mathrm{E}+6 \mathrm{~h}, \\
6.0 / 2.6 \mathrm{E}+6 \mathrm{~h} \\
{[7]}\end{array}$ & $\begin{array}{l}2 / 3.0 \mathrm{E}+2 \mathrm{~d} \\
3 / 6.3 \mathrm{E}+4 \mathrm{~h} \\
10 / 2.7 \mathrm{E}+5 \mathrm{~h} \\
{[2]}\end{array}$ & & & & $\begin{array}{l}8.3 \mathrm{E}-4 / \mathrm{d}(9.1) \\
3.7 \mathrm{E}-6 / \mathrm{h}(8.3)\end{array}$ \\
\hline Fails to run & $\begin{array}{l}242 / 2.4 \mathrm{E}+7 \mathrm{~h} \\
{[15]}\end{array}$ & $\begin{array}{l}0.8 / 8.4 \mathrm{E}+5 \mathrm{~h} \\
1.8 / 2.6 \mathrm{E}+6 \mathrm{~h}, \\
3.7 / 2.6 \mathrm{E}+6 \mathrm{~h} \\
{[7]}\end{array}$ & $\begin{array}{l}2 / 2.0 \mathrm{E}+2 \mathrm{~h}, \\
8 / 6.3 \mathrm{E}+4 \mathrm{~h}, \\
17 / 6.3 \mathrm{E}+4 \mathrm{~h} \\
12\end{array}$ & & & & $9.4 \mathrm{E}-6 / \mathrm{h}(11)$ \\
\hline Overspeed & & $\begin{array}{l}0.2 / 8.4 \mathrm{E}+5 \mathrm{~h} \\
0.4 / 2.6 \mathrm{E}+6 \mathrm{~h}, \\
0.7 / 2.6 \mathrm{E}+6 \mathrm{~h} \\
{[7]}\end{array}$ & & & & & $1.9 \mathrm{E}-6 / \mathrm{h}(11)$ \\
\hline Fails to stop & & & & & & & \\
\hline
\end{tabular}




\begin{tabular}{|c|c|c|c|c|c|c|c|}
\hline \multirow[t]{2}{*}{ System/Component/Failure Mode } & \multicolumn{7}{|c|}{ Failure Data (Category 2 Sources) ${ }^{a}$} \\
\hline & NUCLARR2 & NPRD-3 & OREDA & WIN-330 & TRITIUM & LNG1 & $\begin{array}{c}\text { Aggregated } \\
\text { Results }{ }^{b}\end{array}$ \\
\hline Leakage (external) & $\begin{array}{l}43 / 1.6 \mathrm{E}+9 \mathrm{~h} \\
{[16]}\end{array}$ & $\begin{array}{l}3.6 / 8.4 \mathrm{E}+5 \mathrm{~h}, \\
7.7 / 2.6 \mathrm{E}+6 \mathrm{~h}, \\
15.9 / 2.6 \mathrm{E}+6 \mathrm{~h} \\
{[7]}\end{array}$ & $\begin{array}{l}1 / 6.3 \mathrm{E}+4 \mathrm{~h}, \\
24 / 2.7 \mathrm{E}+5 \mathrm{~h} \\
{[2]}\end{array}$ & & & & $5.9 \mathrm{E}-8 / \mathrm{h}(55)$ \\
\hline Rupture (external) & $\begin{array}{l}2 / 1.6 \mathrm{E}+9 \mathrm{~h} \\
{[17]}\end{array}$ & $\begin{array}{l}0.02 / 8.4 \mathrm{E}+5 \mathrm{~h}, \\
0.05 / 2.6 \mathrm{E}+6 \mathrm{~h}, \\
0.11 / 2.6 \mathrm{E}+6 \mathrm{~h} \\
{[7]}\end{array}$ & $\begin{array}{l}0 / 6.3 \mathrm{E}+4 \mathrm{~h}, \\
0 / 2.7 \mathrm{E}+5 \mathrm{~h} \\
{[2]}\end{array}$ & & & & $1.7 \mathrm{E}-9 / \mathrm{h}(10)$ \\
\hline $\begin{array}{c}\text { Turbine-Driven } \\
\text { Fails to start }\end{array}$ & $\begin{array}{l}72 / 7.1 \mathrm{E}+3 \mathrm{~d} \\
{[18]}\end{array}$ & & & & & & $1.0 \mathrm{E}-2 / \mathrm{d}(10)$ \\
\hline Fails to run & $\begin{array}{l}22 / 2.2 \mathrm{E}+5 \mathrm{~h} \\
{[19]}\end{array}$ & & & & & & $1.0 \mathrm{E}-4 / \mathrm{h}(1.7)$ \\
\hline \multicolumn{8}{|l|}{ Overspeed } \\
\hline \multicolumn{8}{|l|}{ Fails to stop } \\
\hline Leakage (external) & $\begin{array}{l}43 / 1.6 \mathrm{E}+9 \mathrm{~h} \\
{[16]}\end{array}$ & & & & & & $2.7 \mathrm{E}-8 \mathrm{~h}(10)$ \\
\hline Rupture (external) & $2 / 1.6 \mathrm{E}+9 \mathrm{~h}$ & & & & & & $1.6 \mathrm{E}-9 \mathrm{~h}(10)$ \\
\hline $\begin{array}{c}\text { Diesel-Driven } \\
\text { Fails to start }\end{array}$ & $\begin{array}{l}10 / 1.1 \mathrm{E}+3 \mathrm{~d} \\
{[20]}\end{array}$ & & $\begin{array}{l}75 / 1.1 \mathrm{E}+3 \mathrm{~d}, \\
2 / 3.0 \mathrm{E}+2 \mathrm{~d} \\
{[3]}\end{array}$ & & & & $3.4 \mathrm{E}-2 / \mathrm{d}(4.0)$ \\
\hline Fails to ru & & & $\begin{array}{l}4 / 2.0 \mathrm{E}+2 \mathrm{~h} \\
{[3]}\end{array}$ & & & & $2.3 \mathrm{E}-2 \mathrm{~h}(10)$ \\
\hline \multicolumn{8}{|l|}{ Overspeed } \\
\hline \multicolumn{8}{|l|}{ Fails to stop } \\
\hline Leakage (external) & $\begin{array}{l}43 / 1.6 \mathrm{E}+9 \mathrm{~h} \\
{[16]}\end{array}$ & & $\begin{array}{l}1 / 3.8 \mathrm{E}+5 \mathrm{~h} \\
{[3]}\end{array}$ & & & & $2.7 \mathrm{E}-8 \mathrm{~h}(10)$ \\
\hline Rupture (external) & $\begin{array}{l}2 / 1.6 \mathrm{E}+9 \mathrm{~h} \\
{[17]}\end{array}$ & & $\begin{array}{l}0 / 3.8 \mathrm{E}+5 \mathrm{~h} \\
{[3]}\end{array}$ & & & & $1.6 \mathrm{E}-9 / \mathrm{h}(10)$ \\
\hline $\begin{array}{l}\text { Piping/Hose/Jumper } \\
\text { Piping } \\
\text { Leakage (external) }\end{array}$ & $\begin{array}{l}456 ! \\
2.0 \mathrm{E}+11 \mathrm{~h}-\mathrm{ft} \\
{[21]}\end{array}$ & & & & & & $2.3 \mathrm{E}-9 / \mathrm{h}-\mathrm{ft}(10)$ \\
\hline
\end{tabular}


WSRC-TR-93-262

\begin{tabular}{|c|c|c|c|c|c|c|c|}
\hline \multirow[t]{2}{*}{ System/Component/Failure Mode } & \multicolumn{7}{|c|}{ Failure Data (Category 2 Sources) $^{\mathrm{a}}$} \\
\hline & NUCLARR2 & NPRD-3 & OREDA & WIN-330 & TRITUM & LNG1 & $\begin{array}{c}\text { Aggregated } \\
\text { Results }{ }^{b}\end{array}$ \\
\hline Rupture (external) & $\begin{array}{l}111 \\
2.0 \mathrm{E}+11 \mathrm{~h}-\mathrm{ft} \\
{[22]}\end{array}$ & & & & & & $5.8 \mathrm{E}-11 / \mathrm{h}-\mathrm{ft}(10)$ \\
\hline \multicolumn{8}{|l|}{ Plugs } \\
\hline $\begin{array}{l}\text { Hose } \\
\text { Leakage (external) }\end{array}$ & & $\begin{array}{l}01 \\
1.7 \mathrm{E}+7 \mathrm{~h}-\mathrm{ft}, \\
1.91 \\
1.4 \mathrm{E}+9 \mathrm{~h}-\mathrm{ft} \\
{[8]}\end{array}$ & & & & & $1.7 \mathrm{E}-9 / \mathrm{h}-\mathrm{ft}(10)$ \\
\hline Rupture (external) & & $\begin{array}{l}0 / \\
1.7 \mathrm{E}+7 \mathrm{~h}-\mathrm{ft}, \\
14 / \\
1.4 \mathrm{E}+9 \mathrm{~h}-\mathrm{ft} \\
{[8]}\end{array}$ & & & & & $1.0 \mathrm{E}-8 / \mathrm{h}-\mathrm{ft}(10)$ \\
\hline \multicolumn{8}{|l|}{ Plugs } \\
\hline \multicolumn{8}{|l|}{$\begin{array}{l}\text { Jumper } \\
\text { Leakage (external) }\end{array}$} \\
\hline \multicolumn{8}{|l|}{ Rupture (external) } \\
\hline \multicolumn{8}{|l|}{ Plugs } \\
\hline $\begin{array}{l}\text { Vessel } \\
\text { Tank (Unpressurized) } \\
\text { Leakage (external) }\end{array}$ & $\begin{array}{l}8 / 6.3 \mathrm{E}+8 \mathrm{~h} \\
{[23]}\end{array}$ & $\begin{array}{l}7 / 4.3 \mathrm{E}+6 \mathrm{~h} \\
{[9]}\end{array}$ & & & & & $2.2 \mathrm{E}-8 / \mathrm{h}(19)$ \\
\hline Rupture (external) & $\begin{array}{l}1 / 6.3 \mathrm{E}+8 \mathrm{~h} \\
{[24]}\end{array}$ & [9] & & & & & $3.0 \mathrm{E}-9 / \mathrm{h}(19)$ \\
\hline $\begin{array}{l}\text { Tank (Pressurized) } \\
\text { Leakage (external) }\end{array}$ & [23] & $\begin{array}{l}93 / 8.2 \mathrm{E}+6 \mathrm{~h}, \\
565 / 1.2 \mathrm{E}+7 \mathrm{~h} \\
{[10]}\end{array}$ & & & & & $1.0 \mathrm{E}-6 / \mathrm{h}(24)$ \\
\hline Rupture (extermal) & [24] & $\begin{array}{l}3.9 / 8.2 \mathrm{E}+6 \mathrm{~h} \\
24 / 1.2 \mathrm{E}+7 \mathrm{~h} \\
{[10]}\end{array}$ & & & & & $4.5 \mathrm{E}-8 / \mathrm{h}(23)$ \\
\hline $\begin{array}{l}\text { Flange/Gasket } \\
\text { Leakage (external) }\end{array}$ & $\begin{array}{l}116 / 6.3 \mathrm{E}+9 \mathrm{~h} \\
{[25]}\end{array}$ & $16 / 1.2 \mathrm{E}+7 \mathrm{~h}$ & & & & & $2.1 \mathrm{E}-8 / \mathrm{h}(11)$ \\
\hline Rupture (external) & $\begin{array}{l}1 / 6.3 \mathrm{E}+9 \mathrm{~h} \\
{[26]}\end{array}$ & [11] & & & & & $1.8 \mathrm{E}-10 / \mathrm{h}(11)$ \\
\hline
\end{tabular}


WSRC-TR-93-262

\begin{tabular}{|c|c|c|c|c|c|c|c|}
\hline \multirow[t]{2}{*}{ System/Componen//Failure Mode } & \multicolumn{7}{|c|}{ Failure Data (Category 2 Sources) } \\
\hline & NUCLARR2 & NPRD-3 & OREDA & WIN-330 & TRITIUM & LNG1 & $\begin{array}{l}\text { Aggregated } \\
\text { Results }{ }^{b}\end{array}$ \\
\hline \multicolumn{8}{|l|}{$\begin{array}{l}\text { Heat Exchanger } \\
\text { Shell/Tube } \\
\text { Fouling (tubes) }\end{array}$} \\
\hline \multicolumn{8}{|l|}{ Plugs (tubes) } \\
\hline Leakage (ubbes) & $\begin{array}{l}53 / 3.2 \mathrm{E}+8 \mathrm{~h} \\
{[27]}\end{array}$ & & $\begin{array}{l}4 / 6.6 E+4 h \\
{[4]}\end{array}$ & & & & $1.8 \mathrm{E}-7 \mathrm{~h}(17)$ \\
\hline Rupture (tubes) & $\begin{array}{l}2 / 3.2 \mathrm{E}+8 \mathrm{~h} \\
{[28]}\end{array}$ & & & & & & $7.8 \mathrm{E}-9 / \mathrm{h}(10)$ \\
\hline Leakage (shell) & $\begin{array}{l}2 / 3.2 \mathrm{E}+8 \mathrm{~h} \\
{[29]}\end{array}$ & & & & & & $7.8 \mathrm{E}-9 / \mathrm{h}(10)$ \\
\hline Rupture (shell) & $\begin{array}{l}0 / 3.2 \mathrm{E}+8 \mathrm{~h} \\
{[30]}\end{array}$ & & & & & & $1.6 \mathrm{E}-9 / \mathrm{h}(10)$ \\
\hline $\begin{array}{l}\text { Heater (Electrical) } \\
\text { Fails to heat }\end{array}$ & & $\begin{array}{l}1.2 / 8.2 \mathrm{E}+5 \mathrm{~h} \\
{[12]}\end{array}$ & & & & & $1.8 \mathrm{E}-6 / \mathrm{h}(10)$ \\
\hline Overheats & & $\begin{array}{l}0.2 / 8.2 \mathrm{E}+5 \mathrm{~h} \\
{[12]}\end{array}$ & & & & & $3.0 \mathrm{E}-7 / \mathrm{h}(10)$ \\
\hline \multicolumn{8}{|l|}{ Leakage (external) } \\
\hline \multicolumn{8}{|l|}{ Rupture (external) } \\
\hline \multicolumn{8}{|l|}{$\begin{array}{l}\text { Strainer/Filter } \\
\text { Plugs }\end{array}$} \\
\hline Leakage (internal) & & $\begin{array}{l}26 / 1.2 \mathrm{E}+7 \mathrm{~h} \\
{[13]}\end{array}$ & & & & & $2.2 \mathrm{E}-6 / \mathrm{h}(10)$ \\
\hline Rupture (internal) & & $\begin{array}{l}7.7 / 1.2 \mathrm{E}+7 \mathrm{~h} \\
{[13]}\end{array}$ & & & & & $6.8 \mathrm{E}-7 / \mathrm{h}(10)$ \\
\hline \multicolumn{8}{|l|}{$\begin{array}{l}\text { Orifice } \\
\text { Plugs } \\
\end{array}$} \\
\hline $\begin{array}{l}\text { Miscellsmeous } \\
\text { Travelling Screen } \\
\text { Plugs }\end{array}$ & & & & & & & \\
\hline
\end{tabular}

Table 4a. Category 2 data for water system.

a. Failure data are listed as: $\mathrm{X} / \mathrm{Y}$, where $\mathrm{X}$ is the number of failures, and $\mathrm{Y}$ is the exposure period $(\mathrm{h})$ or number of demands. Numbers in brackets refer to notes listed below, arranged by source.

b. Aggregated results are mean and error factor (in parentheses). The error factor is the 95 th percentile/50th percentile. 
Notes

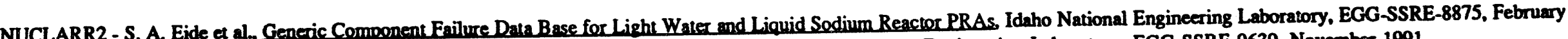
1990. Ans

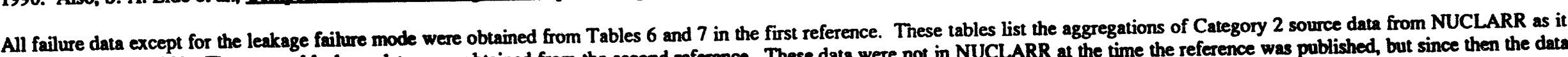

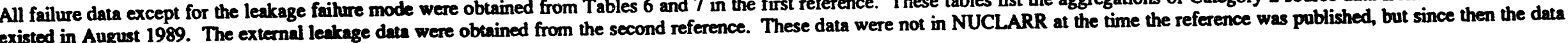
pave been entered into NUCLARR.

1. Manual valve fail to operate data used.

2. Valve extemal leakage data used (second reference).

3. Valve extemal rupture data used (second reference).

4. Check valve fail to operate data used. Data include both fails to open and fails to close.

5. Check valve internal leakage data used. Size of internal leakage not indicated.

6. Motor-operated valve fail to operate, fail to open, and fail to close data used.

7. Moto1-operated valve spurious operation data used.

8. Pneumatic valve fail to operate and fail to open data used.

9. Pneumatic valve spurious operation data used.

10. Pneumatic valve internal leakage data used. Size of internal leakage not indicated.

11. Solenoid valve fail to operate data used.

12. Solenoid valve spurious operation data used.

13. Relief valve fail to open data used.

14. Motor-driven pump fail to start data used.

15. Motor-driven pump fail to run data used.

Table 4a. Category 2 data for water system.

16. Pump external leakage data used (second reference).

17. Pump external rupture data used (second reference).

18. Steam-turbine-driven pump fail to start dats used. 
19. Stearn-turbine-driven pump fail to run data used.

20. Diesel-driven pump fail to start data used.

21. Piping external leakage data used (second reference).

22. Piping extemal rupture data used (second reference).

23. Tank external leakage data used (second refermace).

24. Tank external rupture data used (second reference).

25. Flange external leakage data used (second reference).

26. Flange external rupture data used (second reference).

27. Heat exchanger tube leakage data used (second reference).

28. Heat exchanger tube rupture data used (second reference).

29. Heat exchanger shell leakage data used (second reference).

30. Heat exchanger shell nupture data used (second reference).

NPRD-3 - M. J. Rossi, Nonelectronic Parts Reliability Data, Reliability Analysis Center, Rome Air Development Center, NPRD-3, 1985.

All failure data apply to a ground fixed (GF) environment, sless otherwise indicated. Commercial (C), military (M), and unknown source (?) data were used if available. Failure mode

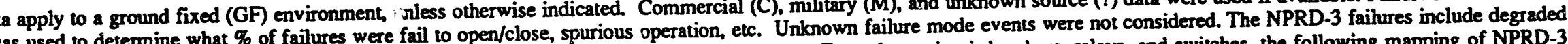

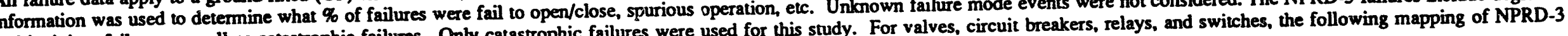
failure modes into the failure modes of interest was used:

Fail to open/close/operate - binding, breach (not for valves), contaminated, cracked/fractured, no movement, no operation, seized, stuck open, and stuck closed

Spurious operation - arcing, burned, collapsed, displaced, false response, improper flow, intermittent, opened, overheated, and shorted

Plugs - none identified

Leakage (intemal) - none identified except for leaking (one-half of events applied to internal leakage for check and relief valves)

Tsibie 4a. Category 2 data for water system.

Leakage (extemal) - leaking

Rupture (extemal) - breach (valves only).

For pumps, fans, and diesel generators, the following mapping was used: 
Fail to start - contaminated, cracked/fractured, no movement, no operation

Fail to run - binding, broken, displaced, false response, improper flow, intermittent, overheated, seized, shorted, stuck closed, stuck open

Overspeed (subset of fail to run) - improper flow (one-half of events used), stuck open

Fail to stop - none identified

Leakage (extemal) - leaking

Rupture (external) - breach (not for generator)

For vessels, piping, hoses, and jumpers, the following mapping was used:

Leakage (external) - cracked/fractured, leaking

Rupture (external) - broken, breach.

For filters, the following mapping was used:

Plug - improper flow (one-half of events used)

Leakage (internal) - improper flow (one-half of events used)

Rupture (intemal) - broken, cracked/fractured, ruptured.

For heaters, the following mapping was used:

Fail to heat - cracked/fractured, no operation, opened

Overheat - shorted.

1. Manually-activated valve data used (p. 153). Failure mode information (p. 297) indicates $25 \%$ of failures are fail to open/close, $50 \%$ are external leakage, and $0 \%$ are external rupture.

2. Check valve data used (p. 150). Failure mode information (p. 296) indicates $8.2 \%$ of failures are fail to close and $2.5 \%$ are fail to open.

3. Check valve data used (p. 150). Failure mode information (p. 296 )

Table 4a. Category 2 data for water system.

4. Check valve data used (p. 150). Failure mode information (p. 296) indicates $0 \%$ of failures are external rupture.

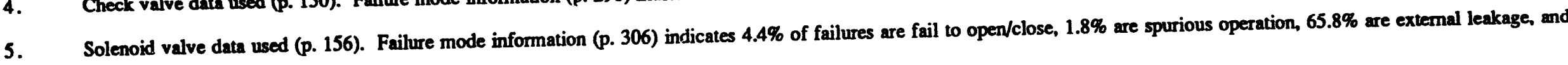




\section{$0 \%$ are external nupture.} Relief valve data used (p. 155). Failure mode information (p. 299)

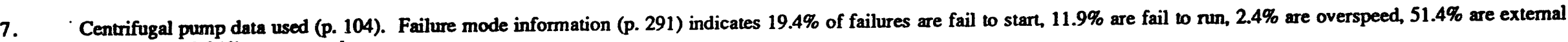
leakage, and $0.34 \%$ are external rupture.

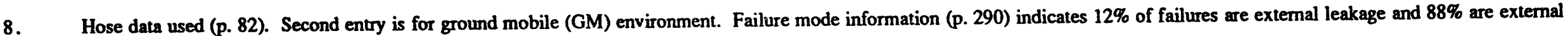
rupture. Each hose was assumed to be $20 \mathrm{ft}$.

9. Storage tank data used (p. 143). No failure mode information, so $100 \%$ assumed to be external leakage/rupture. Size of leakage not indicated.

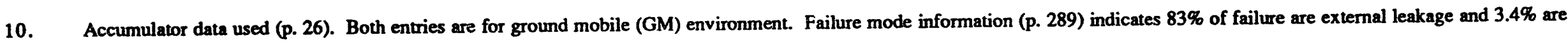
external rupture.

11. General gasket data used (p. 71). No failure mode information, to $100 \%$ assumed to be external leakage/rupture. Size of leakage not indicated.

12. Electrical hearer data used (p. 81). Faihure mode information (p. 287) indicates $62 \%$ of failures are fail to heat and $7.7 \%$ are overheat.

13. Fluid filter das used (p. 67). Failure mode information (p. 285) indicates $75 \%$ of failures are internal leakage and $22 \%$ are internal rupture.

\section{OREDA - Offshore Reliabjlity Data Handbook, Offshore Reliability Data (OREDA), Norway, 1984.}

All failure information was taken from the critical failure mode category, unless otherwise indicated.

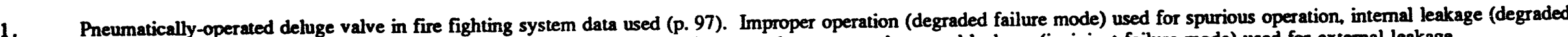
failure mode) used for internal leakage, significant internal leakage used for internal rupture, and external leakage (incipient failure mode) used for external leakage.

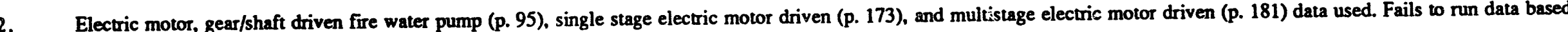
on operational time. Leakage (incipient failure mode) used for external leakage.

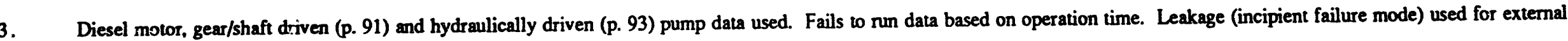
leakage.

4. Water/water tube/shell type heat exchanger data used (p. 207). Leakage (degraded failure mode) used for tube leakage.

WUN-330 - J. N. Wilkinson et al., Idahe Chemical Processing Plant Failure Rate Database, Idaho Natiơndi Engineering Laboratory, WIN-330, October 1991.

All faihure information was taken from Appendix C, p. 32.

Table 4a. Category 2 data for water system.

TRTTUM - L. C. Cadwallader and M. A. Stolpe Gavett, Tritium Waste Treatment System Component Failure Data Analysis from June 18, 1984 to December 31. 1989, Idaho National 
Engineering Laboratory, EGG-FSP-8973, Rev. 1, November 1990; L. C. Cadwallader, M. A. Stolpe Gavett, and L. Quintana, Tritium Room Air Monitor Component Failure Data Analysis 1984 to Necemal Engineering Laboratory, EGG-FSP-9450, May 1991; and L. C. Cadwallader and D. P. Sanchez, Secondary Containment System Component 1992

LNG1 - D. W. Johnson and J. R. Welker, Development of an Improved LNG Plant Failure Rate Data Base, Gas Research Institute, PB82-153503, September 1981.

Aggregated Results 


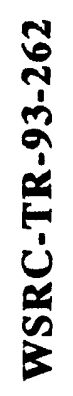

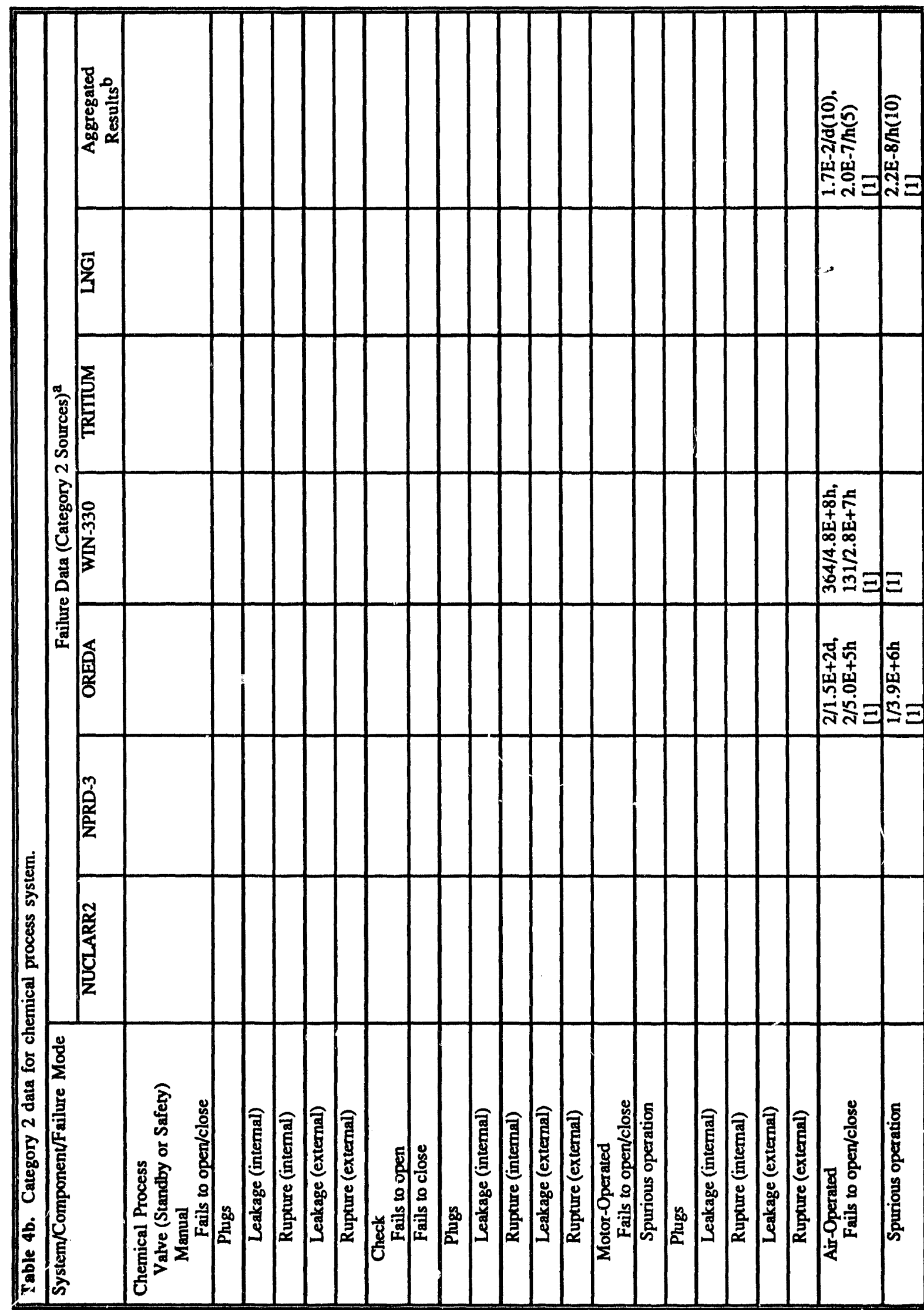




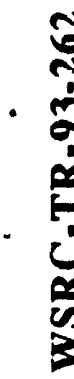

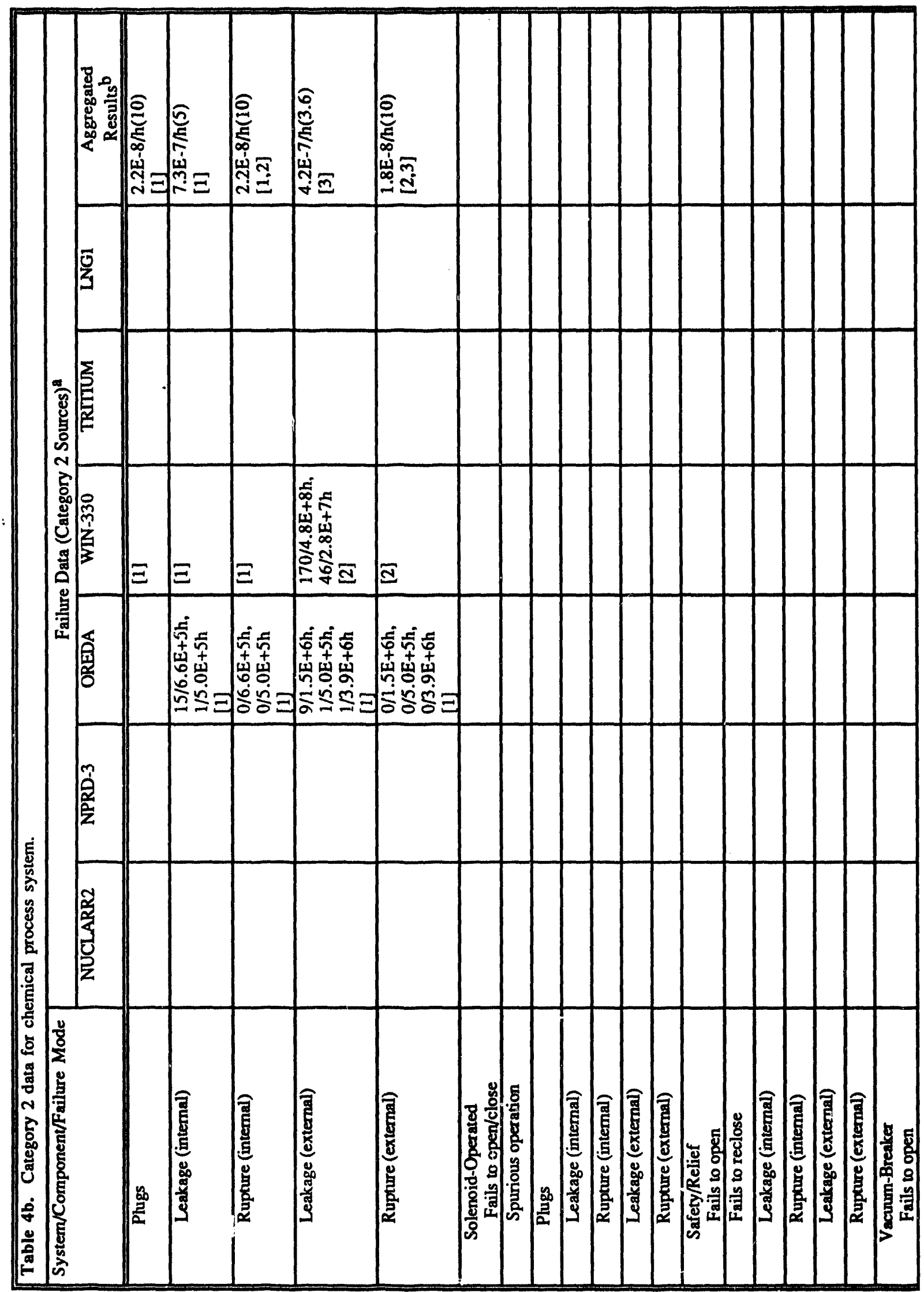


WSRC-TR-93-262

\begin{tabular}{|c|c|c|c|c|c|c|c|}
\hline \multirow[t]{2}{*}{ System/Com; snent/Failure Mode } & \multicolumn{7}{|c|}{ Failure Data (Category 2 Sources) $)^{a}$} \\
\hline & NUCLARR2 & NPRD-3 & OREDA & WIN-330 & TRITIUM & LNG1 & $\begin{array}{c}\text { Aggregated } \\
\text { Results }{ }^{b}\end{array}$ \\
\hline \multicolumn{8}{|l|}{ Fails to reclose } \\
\hline \multicolumn{8}{|l|}{ Leakage (internal) } \\
\hline \multicolumn{8}{|l|}{ Rupture (internal) } \\
\hline \multicolumn{8}{|l|}{ Leakage (external) } \\
\hline \multicolumn{8}{|l|}{ Rupture (external) } \\
\hline \multicolumn{8}{|l|}{$\begin{array}{l}\text { Valve (Control) } \\
\text { Motor-Operated } \\
\text { Fails open } \\
\end{array}$} \\
\hline \multicolumn{8}{|l|}{ Fails closed } \\
\hline \multicolumn{8}{|l|}{ Fails to respond } \\
\hline \multicolumn{8}{|l|}{$\overline{\text { Piugs }}$} \\
\hline \multicolumn{8}{|l|}{ Leakage (external) } \\
\hline \multicolumn{8}{|l|}{ Rupture (external) } \\
\hline $\begin{array}{l}\text { Air-Operated } \\
\text { Fails open }\end{array}$ & & & $\begin{array}{l}3 / 5.8 \mathrm{E}+5 \mathrm{~h} \\
{[2]}\end{array}$ & & & & $6.0 \mathrm{E}-6 / \mathrm{h}(10)$ \\
\hline Fails closed & & & $\begin{array}{l}1 / 5.8 \mathrm{E}+5 \mathrm{~h} \\
{[2]}\end{array}$ & & & & $2.6 \mathrm{E}-6 / \mathrm{h}(10)$ \\
\hline \multicolumn{8}{|l|}{ Fails to respond } \\
\hline \multicolumn{8}{|l|}{ Plugs } \\
\hline Leakage (external) & & & $\begin{array}{l}6 / 5.8 \mathrm{E}+5 \mathrm{~h} \\
{[2]}\end{array}$ & & & & $1.1 \mathrm{E}-5 / \mathrm{h}(10)$ \\
\hline \multicolumn{8}{|l|}{ Rupture (external) } \\
\hline \multicolumn{8}{|l|}{$\begin{array}{l}\text { Solenoid-Operated } \\
\text { Fails open }\end{array}$} \\
\hline \multicolumn{8}{|l|}{ Fails closed } \\
\hline \multicolumn{8}{|l|}{ Fails to respond } \\
\hline \multicolumn{8}{|l|}{ Phugs } \\
\hline Leakage (external) & & & & & & & \\
\hline
\end{tabular}




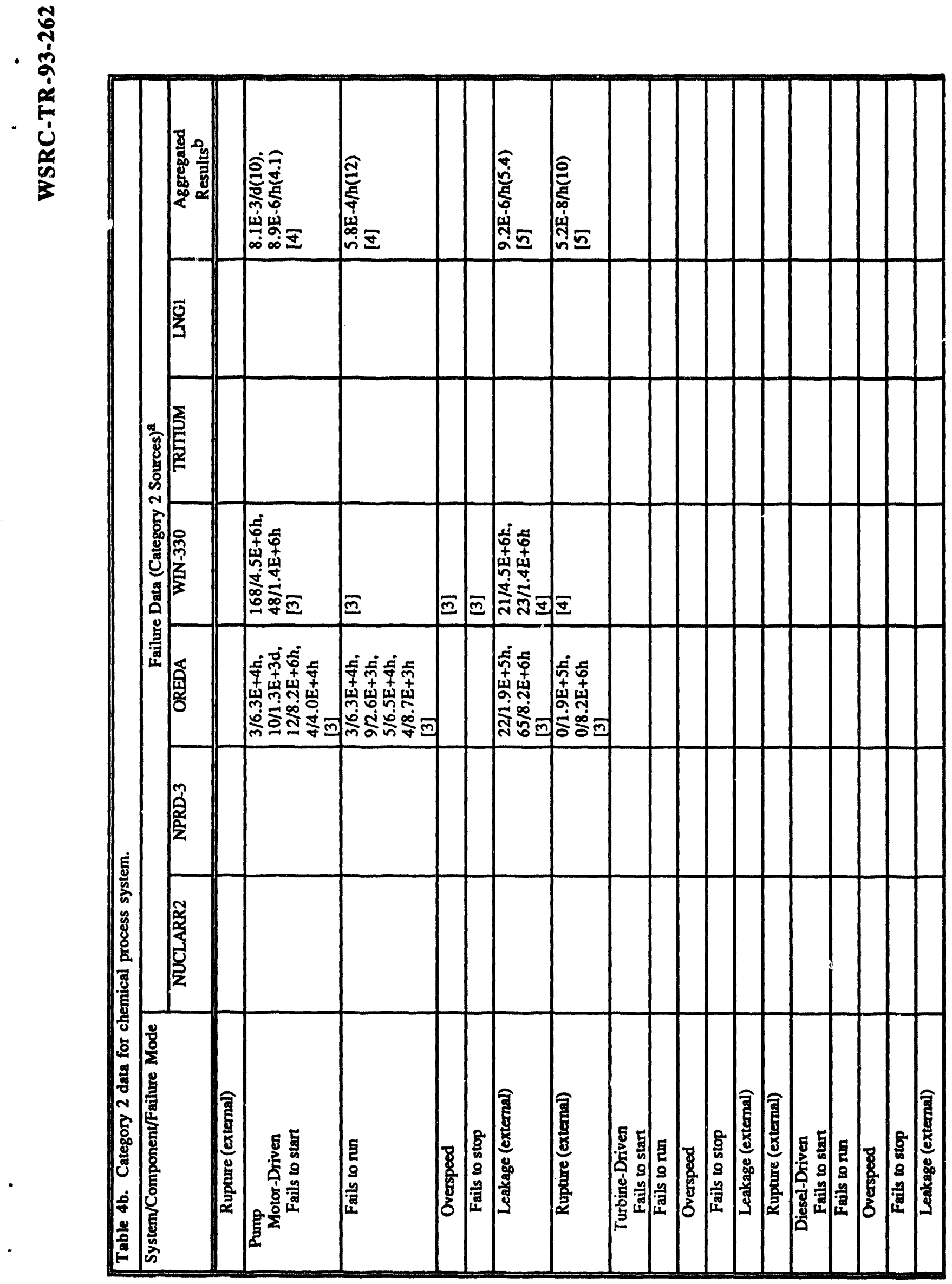


Table 4b. Category 2 data for chemical process system.

\begin{tabular}{|c|c|c|c|c|c|c|c|}
\hline \multirow[t]{2}{*}{ System/Component/Failure Mode } & \multicolumn{7}{|c|}{ Failure Data (Category 2 Sources) ${ }^{a}$} \\
\hline & NUCLARR2 & FPRD-3 & OREDA & WIN-330 & TRTIUM & LNG1 & $\begin{array}{l}\text { Aggregated } \\
\text { Results }^{\mathrm{b}}\end{array}$ \\
\hline \multicolumn{8}{|l|}{ Rupture (external) } \\
\hline \multicolumn{8}{|l|}{$\begin{array}{l}\text { Piping/Hose/Jumper } \\
\text { Piping } \\
\text { Leakage (external) }\end{array}$} \\
\hline \multicolumn{8}{|l|}{ Rupture (external) } \\
\hline \multicolumn{8}{|l|}{ Plugs } \\
\hline \multicolumn{8}{|l|}{$\begin{array}{l}\text { Hose } \\
\text { Leakage (external) }\end{array}$} \\
\hline \multicolumn{8}{|l|}{ Rupture (external) } \\
\hline \multicolumn{8}{|l|}{ Plugs } \\
\hline \multicolumn{8}{|l|}{$\begin{array}{l}\text { Jumper } \\
\text { Leakage (external) }\end{array}$} \\
\hline \multicolumn{8}{|l|}{ Rupture (external) } \\
\hline \multicolumn{8}{|l|}{ Plugs } \\
\hline $\begin{array}{l}\text { Vessel } \\
\text { Tank (Unpressurized) } \\
\text { Leakage (external) }\end{array}$ & & & & $\begin{array}{l}13 / 4.6 \mathrm{E}+6 \mathrm{~h}, \\
19 / 8.0 \mathrm{E}+6 \mathrm{~h} \\
{[5]}\end{array}$ & & & $2.6 \mathrm{E}-6 / \mathrm{h}(10)$ \\
\hline Rupture (external) & & & & [5] & & & \\
\hline $\begin{array}{l}\text { Tank (Pressurized) } \\
\text { Leakage (external) }\end{array}$ & & & $\begin{array}{l}24 / 1.7 \mathrm{E}+6 \mathrm{~h} \\
{[4]}\end{array}$ & $\begin{array}{l}13 / 4.6 \mathrm{E}+6 \mathrm{~h}, \\
19 / 8.0 \mathrm{E}+6 \mathrm{~h} \\
{[5]}\end{array}$ & & & $\begin{array}{l}3.7 \mathrm{E}-6 / \mathrm{h}(3.8) \\
{[5]}\end{array}$ \\
\hline Rupture (external) & & & $\begin{array}{l}2 / 1.7 \mathrm{E}+6 \mathrm{~h} \\
{[4]}\end{array}$ & [5] & & & $\begin{array}{l}3.8 \mathrm{E}-7 / \mathrm{h}(10) \\
{[5]}\end{array}$ \\
\hline \multicolumn{8}{|l|}{$\begin{array}{l}\text { Flange/Gasket } \\
\text { Leakage (external) }\end{array}$} \\
\hline Rupture (extermal) & & & & & & & \\
\hline $\begin{array}{l}\text { Heat Exchanger } \\
\text { Shell/Tube } \\
\text { Fouling (tubes) }\end{array}$ & & & $\begin{array}{l}13 / 2.3 \mathrm{E}+6 \mathrm{~h} \\
{[5]}\end{array}$ & $\begin{array}{l}31 / 1.6 \mathrm{E}+6 \mathrm{~h}, \\
15 / 8.4 \mathrm{E}+5 \mathrm{~h} \\
{[6]}\end{array}$ & & & $\begin{array}{l}7.8 \mathrm{E}-6 / \mathrm{h}(1.3) \\
{[6]}\end{array}$ \\
\hline Phigs (ubes) & & & & [6] & & & \\
\hline Leakage (tubes) & & & $\begin{array}{l}12 / 2.3 \mathrm{E}+6 \mathrm{~h}, \\
1 / 2.2 \mathrm{E}+4 \mathrm{~h} \\
{[5]}\end{array}$ & [6] & & & $\begin{array}{l}7.2 \mathrm{E}-6 / \mathrm{h}(1.3) \\
{[6]}\end{array}$ \\
\hline Rupture (tubes) & & & & [6] & & & \\
\hline
\end{tabular}


WSRC-TR-93-262

\begin{tabular}{|c|c|c|c|c|c|c|c|}
\hline \multirow[t]{2}{*}{ System/Component/Failure Mode } & \multicolumn{7}{|c|}{ Failure Data (Category 2 Sources) ${ }^{a}$} \\
\hline & NUCLARR2 & NPRD-3 & $\overline{\text { OREDA }}$ & WIN-330 & TRITIUM & LNG1 & $\begin{array}{l}\text { Aggregated } \\
\text { Results }\end{array}$ \\
\hline Leakage (shell) & & & & [6] & & & \\
\hline Rupture (shell) & & & & [6] & & & \\
\hline $\begin{array}{l}\text { Heater (Electrical) } \\
\text { Fails to heat }\end{array}$ & & & $\begin{array}{l}4 / 2.2 \mathrm{E}+4 \mathrm{~h}, \\
1 / 1.2 \mathrm{E}+5 \mathrm{~h} \\
{[6]}\end{array}$ & & & & $3.5 \mathrm{E}-5 / \mathrm{h}(5.8)$ \\
\hline Overheats & & & $\begin{array}{l}1 / 2.2 \mathrm{E}+4 \mathrm{~h} \\
{[6]}\end{array}$ & & & & $\begin{array}{l}7.0 \mathrm{E}-6 / \mathrm{h}(10) \\
{[7]}\end{array}$ \\
\hline \multicolumn{8}{|l|}{ Leakage (external) } \\
\hline \multicolumn{8}{|l|}{ Rupture (external) } \\
\hline $\begin{array}{l}\text { Strainer/Filter } \\
\text { Plugs }\end{array}$ & & $\begin{array}{l}1.2 / 5.8 \mathrm{E}+6 \mathrm{~h} \\
{[1]}\end{array}$ & $\begin{array}{l}19 / 6.4 \mathrm{E}+5 \mathrm{~h} \\
{[7]}\end{array}$ & & & & $3.1 \mathrm{E}-6 / \mathrm{h}(11)$ \\
\hline Leakage (internal) & & $\begin{array}{l}2.7 / 5.8 \mathrm{E}+6 \mathrm{~h} \\
\text { [1] }\end{array}$ & & & & & $\begin{array}{l}7.0 \mathrm{E}-6 / \mathrm{h}(11) \\
{[8]}\end{array}$ \\
\hline Rupture (internal) & & $\begin{array}{l}0 / 5.8 \mathrm{E}+6 \mathrm{~h} \\
{[1]}\end{array}$ & & & & & $\begin{array}{l}7.0 \mathrm{E}-7 / \mathrm{h}(11) \\
{[2,8]}\end{array}$ \\
\hline \multicolumn{8}{|l|}{$\begin{array}{c}\text { Orifice } \\
\text { Phugs }\end{array}$} \\
\hline \multicolumn{8}{|l|}{$\begin{array}{l}\text { Miscellaneous } \\
\text { Mixer/Blender } \\
\text { Failure }\end{array}$} \\
\hline $\begin{array}{l}\text { Agitator } \\
\text { Failure }\end{array}$ & & & & $\begin{array}{l}4 / 6.3 \mathrm{E}+5 \mathrm{~h}, \\
4 / 6.8 \mathrm{E}+5 \mathrm{~h} \\
{[7]}\end{array}$ & & & $6.5 \mathrm{E}-6 / \mathrm{h}(10)$ \\
\hline $\begin{array}{l}\text { Centrifuge } \\
\text { Failure }\end{array}$ & & & & & & & \\
\hline
\end{tabular}

Table 4b. Category 2 data for chemical process system.

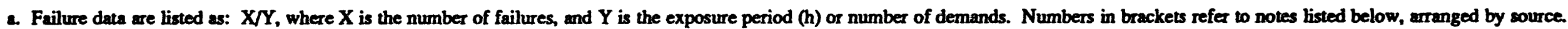

b. Aggregated results are mean and error factor (in parentheses). The error factor is the 95 th percentile/50th percentile. 


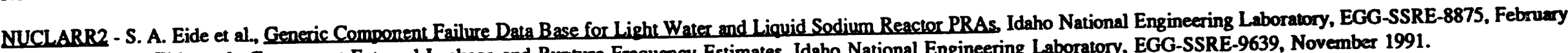
1990. Also, S. A. Eide et al., Component External Leakage and Rupture Frequency Estimates, Idaho National Engineering Laboratory, EGG-SSRE-9639, November 1991.

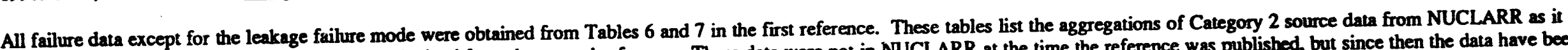

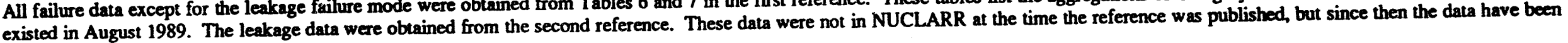
entered into NUCLARR.

NPRD-3 - M. J. Rossi, Nonelectronic Parts Reliability Data, Reliability Analysis Center, Rome Air Development Center, NPRD-3, 1985.

Ill failure data apply to a ground fixed (GF) environment, unless otherwise indicated. Commercial (C), military (M), and unknown source (?) data were used if available. Failure mode apply to a greund fixed (GF) encente were not considered. The NPRD-3 failures include degraded

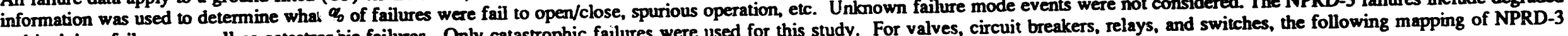

and incipient failures as well as catastrojic failures. Only
failure moves into the failure modes of interest was used:

Fail to open/close/operate - binding, breach (not for valves), contaminated. cracked/fractured, no movement, no operation, seized, stuck open, and stuck closed

Spurious operation - arcing, burned, collapsed, displaced, false response, improper flow, intermittent, opened, overheated, and shorted

Plugs - none identified

Leakage (internal) - none identified except for leaking (one-half of events applied to intemal leakage for check and relief valves)

Leakage (external) - leaking

Rupture (external) - breach (valves only).

For pumps, fans, and diesel generators, the following mapping was used:

Fail to start - contaminated, cracked/fractured, no movement, no operation

Fail to run - binding, broken, displaced, false response, improper flow, intermittent, overheated, seized, shorted, stuck closed, stuck open

Overspeed (subset of fail to run) - improper flow (one-half of events used), stuck open

Fail $\rightarrow$ stop - none identified

Leakage (extemal) - leaking

Table 4b. Category 2 data for chemical process system.

Rupture (external) - breach (not for generator) 


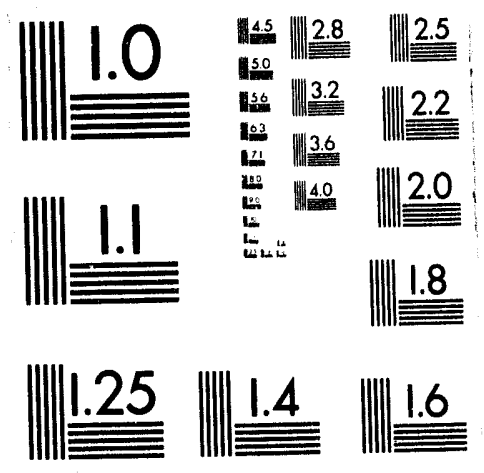



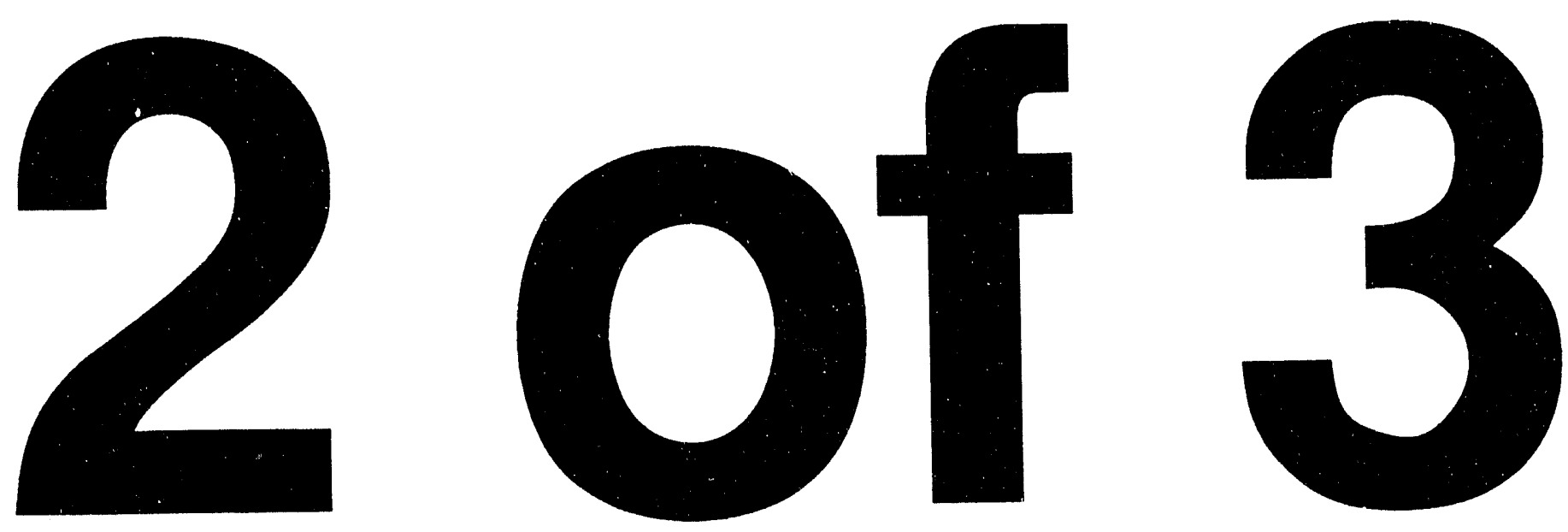
For vessels, piping, hoses, and jumpers, the following mapping was used:

Leakage (cxternal) - cracked/fractured, leaking

Rupture (external) - broken, breach.

For filters, the following mapping was used:

Plug - improper flow (one-half of events used)

Leakage (internal) - improper flow (one-half of events used)

Rupture (internal) - broken, cracked/fractured, ruptured.

For heaters, the following mapping was used:

Fail to heat - cracked/fractured, no operation, opened

Overheat - shorted.

1. Gas filter data used (p. 68). Failure mode information (p. 286) indicates $16.7 \%$ of failures are plugs, $38.9 \%$ are internal leakage, and $0 \%$ are internal ruparre.

OREDA - Offshore Reliability Data Handbook, Offshore Reliability Data (OREDA), Norway, 1984.

All failure information was taken from the critical failure mode category, unless otherwise indicated.

1.

Hydraulically and pneumatically operated valve (oil fluid) data used (pp. 111, 117, and 159). Improper operation (degraded failure mod
leakare (degraded failure mode) data used for internal leakage, and external leakage (degraded failure mode) used for external leakage.

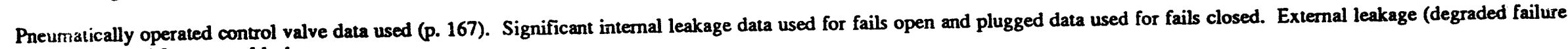
mode) data used for external leakage.

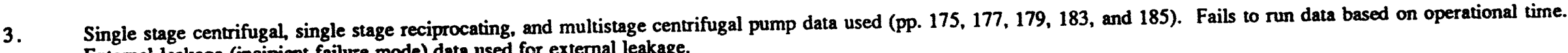
External leakage (incipient failure mode) data used for external leakage.

4. Separator data used (p. 143). Minor leakage (incipient failure mode) data used for external leakage, and major leakage and break data used for external rupture.

5. Oil/water (p. 193) and glycol/water (p. 203) tube/shell heat exchanger data used. Leakage (degraded failure mode) data used for tube leakage.

6. Oil (p. 215) and glycol (p. 219) heater data used.

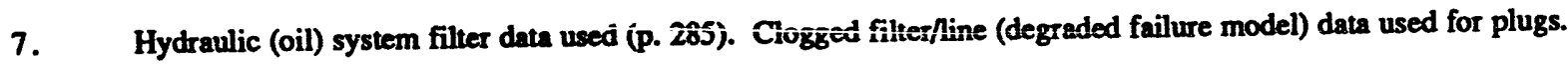


Table 4b. Category 2 data for chemical process system.

WIN-330 - J. N. Wilkinson et al., Idaho Chemical Processine Plant Failure Rate Database, Idaho National Engineering Laboratory, WIN-330, October 1991.

All failure information was taken from Appendix C. p. 32.

1. Valve (non-acid and acid fluid) data used (other failure mode). All valves assumed to be air-operated. Failure modes (other than leak) not indicated.

2. See Note 1. Leak failure mode data used for external leakage/rupture.

3. Pump (non-acid and acid fluid) data used (other failure mode). All pumps assumed to be motor-operated. Failure modes (other than leak) not indicated.

4. See Note 3. Leak failure mode data used for external leakage/rupture.

5. Vessel (non-acid and acid fluid) data used for unpressurized and pressurized tanks. All failure mode data used for external leakage/rupture.

6. Heat exchanger/condenser (non-acid and acid fluid) data used. Failure modes not indicated.

7. Agitator (non-acid and acid fluid) data used. Faihure modes not indicated.

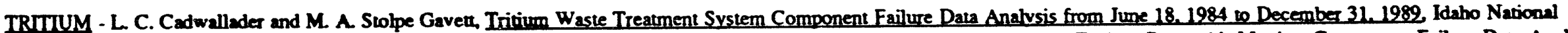

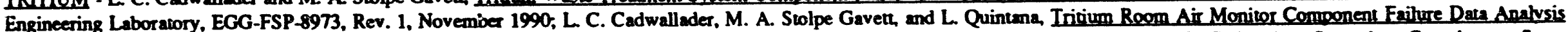

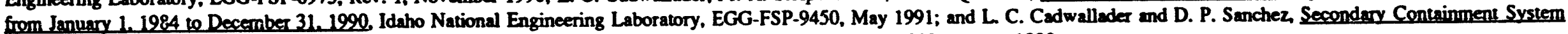
Component Faihure Data Analysis from 1984 to 1991. Idaho Nationel Engineering Laboratory. EGG-FSP-10323, August 1992.

LNG1 - D. W. Johnson and J. R. Welker, Development of an Improved LNG Plant Failure Rate Data Base, Gas Research Institute, PB82-153503, September 1981.

\section{Acoregated Results}

1. All failure data were combined to obtain an overall failure rate. The OREDA data were used to estimate the fraction for each failure mode. Data indicate 4 fails to open/close, 16 internal leakages, and zero spurious operations, plugs, and internal ruptures. Zero failure cases were handled using a Bayesian update of a noninformative prior with zero events in 20 "demands".

2. Failure rate based on zero failures and may be conservatively high.

3. Similar to Note 1. Ruptures are zero events in 11 "demands".

4. One-half of WIN-330 failures were assigned to fails to start. Fails to run result was increased by the same amount that the fails to start estimate was increased.

5. Leakage/rupture combined result was calculated. Then OREDA data were used to partition into extemal leakage and extemal rupture.

6. Combined fouling and tube leakage result was calculated. Then OREDA data were used to partition into fouling and tube leakage.

7. One-fifth of fails to heat result used, based on OREDA data. 
Plugs result multiplied by $2.7 / 1.2$ to obtain internal leakage result, and by $1 /[2(1.2)+2]$ to obtain rupture result, based on NPRD-3 data. 
WSRC-TR-93-262

\begin{tabular}{|c|c|c|c|c|c|c|c|}
\hline \multirow[t]{2}{*}{ System/Component/Failure Mode } & \multicolumn{7}{|c|}{ Failure Data (Category 2 Sources) } \\
\hline & NUCLARR2 & NPRD-3 & $\overline{\text { OREDA }}$ & WIN-330 & TRITIUM & LNG1 & $\begin{array}{c}\text { Aggregated } \\
\text { Results }\end{array}$ \\
\hline $\begin{array}{l}\text { Compressed Gas } \\
\text { Valve (Standby or Safety) } \\
\text { Manual } \\
\text { Fails to open/close } \\
\end{array}$ & & $\begin{array}{l}0 / 8.3 \hat{E}+5 h \\
{[1]}\end{array}$ & & & $\begin{array}{l}3 / 1.6 \mathrm{E}+3 \mathrm{~d} \\
{[1]}\end{array}$ & & $\begin{array}{l}2.2 \mathrm{E}-3 / \mathrm{d}(10) \\
6.0 \mathrm{E}-7 / \mathrm{h}(10)\end{array}$ \\
\hline \multicolumn{8}{|l|}{ Plugs } \\
\hline \multicolumn{8}{|l|}{ Leakage (internal) } \\
\hline \multicolumn{8}{|l|}{ Rupture (internal) } \\
\hline Leakage (external) & & $\begin{array}{l}0 / 8.3 \mathrm{E}+5 \mathrm{~h} \\
{[1]}\end{array}$ & & & $\begin{array}{l}0 / 3.9 \mathrm{E}+6 \mathrm{~h} \\
{[1]}\end{array}$ & & $1.1 \mathrm{E}-7 / \mathrm{h}(10)$ \\
\hline Rupture (external) & & [1] & & & [1] & & \\
\hline $\begin{array}{l}\text { Check } \\
\text { Fails to open }\end{array}$ & & $\begin{array}{l}4.1 / 1.6 \mathrm{E}+6 \mathrm{~h} \\
{[2]}\end{array}$ & $\begin{array}{l}1 / 3.1 \mathrm{E}+6 \mathrm{~h} \\
{[1]}\end{array}$ & & & & $1.1 \mathrm{E}-6 / \mathrm{h}(2.7)$ \\
\hline Fails to close & & $\begin{array}{l}8.9 / 1.6 \mathrm{E}+6 \mathrm{~h} \\
{[2]}\end{array}$ & & & & & $\begin{array}{l}2.4 \mathrm{E}-6 / \mathrm{h}(2.7) \\
{[1]}\end{array}$ \\
\hline \multicolumn{8}{|l|}{ Phugs } \\
\hline Leakage (internal) & & $13 / 1.6 \mathrm{E}+6 \mathrm{~h}$ & & & & & $8.4 \mathrm{E}-6 / \mathrm{h}(10)$ \\
\hline Rupture (internal) & & [3] & $\begin{array}{l}1 / 3.1 \mathrm{E}+6 \mathrm{~h} \\
{[1]}\end{array}$ & & & & $4.8 \mathrm{E}-7 / \mathrm{h}(10)$ \\
\hline Leakage (external) & & $\begin{array}{l}13 / 1.6 \mathrm{E}+6 \mathrm{~h} \\
{[3]}\end{array}$ & & & & & $8.4 \mathrm{E}-6 / \mathrm{h}(10)$ \\
\hline Rupture (external) & & $\begin{array}{l}0 / 1.6 \mathrm{E}+6 \mathrm{~h} \\
{[4]}\end{array}$ & & & & & $\begin{array}{l}3.1 \mathrm{E}-7 / \mathrm{h}(10) \\
{[2]}\end{array}$ \\
\hline $\begin{array}{l}\text { Motor-Operated } \\
\text { Fails to open/close }\end{array}$ & & & & & $\begin{array}{l}10 / 2.0 \mathrm{E}+4 \mathrm{~d} \\
{[2]}\end{array}$ & & $5.3 \mathrm{E}-4 / \mathrm{d}(10)$ \\
\hline \multicolumn{8}{|l|}{ Spurious operation } \\
\hline \multicolumn{8}{|l|}{ Plugs } \\
\hline \multicolumn{8}{|l|}{ Leakage (internal) } \\
\hline \multicolumn{8}{|l|}{ Rupture (internal) } \\
\hline Leakage (external) & & & & & $\begin{array}{l}0 / 3.3 \mathrm{E}+6 \mathrm{~h} \\
{[2]}\end{array}$ & & $1.5 \mathrm{E}-7 / \mathrm{h}(10)$ \\
\hline Rupture (external) & & & & & & & \\
\hline
\end{tabular}


WSRC-TR-93-262

\begin{tabular}{|c|c|c|c|c|c|c|c|}
\hline \multirow[t]{2}{*}{ System/Component/Failure Mode } & \multicolumn{7}{|c|}{ Failure Data (Category 2 Sources) ${ }^{\mathrm{a}}$} \\
\hline & NUCLARR2 & NPRD-3 & OREDA & WIN-330 & TRITIUM & LNG1 & $\begin{array}{l}\text { Aggregated } \\
\text { Results }^{b}\end{array}$ \\
\hline $\begin{array}{l}\text { Air-Operated } \\
\text { Fails to open/close }\end{array}$ & & $\begin{array}{l}0.6 / 1.8 \mathrm{E}+7 \mathrm{~h} \\
{[5]}\end{array}$ & $\begin{array}{l}13 / 6.0 \mathrm{E}+6 \mathrm{~h}, \\
13 / 4.2 \mathrm{E}+6 \mathrm{~h}, \\
9 / 6.7 \mathrm{E}+5 \mathrm{~h}, \\
2 / 5.1 \mathrm{E}+5 \mathrm{~h}, \\
12 / 2.7 \mathrm{E}+3 \mathrm{~d}, \\
15 / 6.2 \mathrm{E}+3 \mathrm{~d} \\
{[2]}\end{array}$ & & & & $\begin{array}{l}3.0 \mathrm{E}-3 / \mathrm{d}(1.2) \\
1.3 \mathrm{E}-6 / \mathrm{h}(6.5)\end{array}$ \\
\hline Spurious operation & & $\begin{array}{l}0.3 / 1.8 \mathrm{E}+7 \mathrm{~h} \\
{[5]}\end{array}$ & $\begin{array}{l}27 / 2.3 \mathrm{E}+6 \mathrm{~h}, \\
15 / 2.2 \mathrm{E}+6 \mathrm{~h} \\
{[2]}\end{array}$ & & & & $1.9 \mathrm{E}-6 / \mathrm{h}(8.1)$ \\
\hline$\overline{\text { Plugs }}$ & & & $\begin{array}{l}1 / 2.3 \mathrm{E}+6 \mathrm{~h} \\
{[2]}\end{array}$ & & & & $6.5 \mathrm{E}-7 \mathrm{~h}(10)$ \\
\hline Leakage (internal) & & & $\begin{array}{l}9 / 6.0 \mathrm{E}+6 \mathrm{~h}, \\
8 / 4.2 \mathrm{E}+6 \mathrm{~h} \\
14 / 2.3 \mathrm{E}+6 \mathrm{~h} \\
{[2]}\end{array}$ & & & & $2.5 \mathrm{E}-6 / \mathrm{h}(2.5)$ \\
\hline Rupture (internal) & & & $\begin{array}{l}0 / 6.0 \mathrm{E}+6 \mathrm{~h}, \\
0 / 4.2 \mathrm{E}+6 \mathrm{~h}, \\
4 / 2.3 \mathrm{E}+6 \mathrm{~h}, \\
2 / 2.2 \mathrm{E}+6 \mathrm{~h} \\
{[2]}\end{array}$ & & & & $4.1 \mathrm{E}-7 \mathrm{~h}(4.8)$ \\
\hline Leakage (external) & & $\begin{array}{l}9.2 / 1.8 \mathrm{E}+7 \mathrm{~h} \\
{[5]}\end{array}$ & $\begin{array}{l}10 / 4.2 \mathrm{E}+6 \mathrm{~h}, \\
19 / 2.3 \mathrm{E}+6 \mathrm{~h} \\
{[2]}\end{array}$ & & & & $1.6 \mathrm{E}-6 / \mathrm{h}(5.5)$ \\
\hline Rupture (external) & & $\begin{array}{l}0 / 1.8 \mathrm{E}+7 \mathrm{~h} \\
{[5]}\end{array}$ & $\begin{array}{l}0 / 4.2 \mathrm{E}+6 \mathrm{~h}, \\
0 / 2.3 \mathrm{E}+6 \mathrm{~h} \\
{[2]}\end{array}$ & & & & $\begin{array}{l}2.0 \mathrm{E}-8 / \mathrm{h}(10) \\
{[2]}\end{array}$ \\
\hline $\begin{array}{l}\text { Solenoid-Operated } \\
\text { Fails to open/close }\end{array}$ & & $\begin{array}{l}0.8 / 1.9 \mathrm{E}+6 \mathrm{~h} \\
{[6]}\end{array}$ & & & & & $6.8 \mathrm{E}-7 / \mathrm{h}(10)$ \\
\hline Spurious operation & & $\begin{array}{l}0.3 / 1.9 \mathrm{E}+6 \mathrm{~h} \\
{[6]}\end{array}$ & & & & & $4.2 \mathrm{E}-7 / \mathrm{h}(10)$ \\
\hline \multicolumn{8}{|l|}{ Plugs } \\
\hline \multicolumn{8}{|l|}{ Leakage (internal) } \\
\hline \multicolumn{8}{|l|}{ Rupture (internal) } \\
\hline Leakage (external) & & $12.5 / 1.9 \mathrm{E}+6 \mathrm{~h}$ & & & & & $5.8 \mathrm{E} \cdot 6 / \mathrm{h}(10)$ \\
\hline
\end{tabular}


WSRC-TR-93-262

\begin{tabular}{|c|c|c|c|c|c|c|c|}
\hline \multirow[t]{2}{*}{ System/Component/Failure Mode } & \multicolumn{7}{|c|}{ Failure Data (Category 2 Sources) } \\
\hline & NUCLARR2 & NPRD-3 & OREDA & WIN-330 & TRITTUM & $\overline{\text { LNG1 }}$ & $\begin{array}{l}\text { Aggrizgated } \\
\text { Resialts }{ }^{b}\end{array}$ \\
\hline Rupture (external) & & $\begin{array}{l}0 / 1.9 \mathrm{E}+6 \mathrm{~h} \\
{[6]}\end{array}$ & & & & & $\begin{array}{l}2.6 \mathrm{E}-7 / \mathrm{h}(10) \\
{[2]}\end{array}$ \\
\hline \multicolumn{8}{|l|}{$\begin{array}{l}\text { Safety/Relief } \\
\text { Fails to open }\end{array}$} \\
\hline \multicolumn{8}{|l|}{ Fails to reclose } \\
\hline Leakage (internal) & & $3.5 / 4.3 \mathrm{E}+6 \mathrm{~h}$ & & & & & $9.3 \mathrm{E}-7 / \mathrm{h}(10)$ \\
\hline Rupture (internal) & & {$[7]$} & & & & & \\
\hline Leakage (external) & & $\begin{array}{l}3.5 / 4.3 \mathrm{E}+6 \mathrm{~h} \\
{[7]}\end{array}$ & & & & & $9.3 \mathrm{E}-7 / \mathrm{h}(10)$ \\
\hline Rupture (external) & & $\begin{array}{l}0 / 4.3 \mathrm{E}+6 \mathrm{~h} \\
{[8]}\end{array}$ & & & & & $\begin{array}{l}1.2 \mathrm{E}-7 / \mathrm{h}(10) \\
{[2]}\end{array}$ \\
\hline \multicolumn{8}{|l|}{$\begin{array}{l}\text { Vacuum-Breaker } \\
\text { Fails to open }\end{array}$} \\
\hline \multicolumn{8}{|l|}{ Fails to rectose } \\
\hline \multicolumn{8}{|l|}{ Leakage (internal) } \\
\hline \multicolumn{8}{|l|}{ Rupture (internal) } \\
\hline \multicolumn{8}{|l|}{ Leakage (externsl) } \\
\hline \multicolumn{8}{|l|}{ Rupture (extermal) } \\
\hline \multicolumn{8}{|l|}{$\begin{array}{l}\text { Valve (Control) } \\
\text { Motor-Operated } \\
\text { Fails open }\end{array}$} \\
\hline \multicolumn{8}{|l|}{ Fails closed } \\
\hline \multicolumn{8}{|l|}{ Fails to respond } \\
\hline \multicolumn{8}{|l|}{ Phugs } \\
\hline \multicolumn{8}{|l|}{ Leakage (external) } \\
\hline \multicolumn{8}{|l|}{ Rupture (external) } \\
\hline $\begin{array}{l}\text { Air-Operated } \\
\text { Fails open }\end{array}$ & & & $\begin{array}{l}1 / 2.3 \mathrm{E}+6 \mathrm{~h} \\
5 / 1.2 \mathrm{E}+6 \mathrm{~h} \\
{[3]}\end{array}$ & & $\begin{array}{l}0 / 4.3 \mathrm{E}+5 \mathrm{~h} \\
29 / 6.9 \mathrm{E}+5 \mathrm{~h} \\
{[3]}\end{array}$ & & $\begin{array}{l}3.0 \mathrm{E}-6 / \mathrm{h}(6.1) \\
{[3]}\end{array}$ \\
\hline
\end{tabular}


WSRC-TR-93-262

\begin{tabular}{|c|c|c|c|c|c|c|c|}
\hline \multirow[t]{2}{*}{ System/Component/Failure Mode } & \multicolumn{7}{|c|}{ Failure Data (Category 2 Sources) ${ }^{\mathbf{a}}$} \\
\hline & NUCLARR2 & NPRD-3 & $\overline{\text { OREDA }}$ & WIN-330 & TRITIUM & LNG1 & $\begin{array}{l}\text { Aggregated } \\
\text { Results }{ }^{b}\end{array}$ \\
\hline Finis closed & & & & & [3] & & $\begin{array}{l}3.0 \mathrm{E}-6 / \mathrm{h}(6.1) \\
{[3]}\end{array}$ \\
\hline Fails to respond & & & $\begin{array}{l}3 / 2.3 \mathrm{E}+6 \mathrm{~h}, \\
3 / 1.2 \mathrm{E}+6 \mathrm{~h} \\
\text { [3] }\end{array}$ & & [3] & & $\begin{array}{l}3.0 \mathrm{E}-6 / \mathrm{h}(6.1) \\
{[3]}\end{array}$ \\
\hline \multicolumn{8}{|l|}{ Plugs } \\
\hline Leakage (external) & & & $\begin{array}{l}3 / 2.3 \mathrm{E}+6 \mathrm{~h} \\
14 / 1.2 \mathrm{E}+6 \mathrm{~h} \\
{[3]}\end{array}$ & & & & $4.9 \mathrm{E}-6 / \mathrm{h}(3.7)$ \\
\hline Rupture (external) & & & $\begin{array}{l}0 / 2.3 \mathrm{E}+6 \mathrm{~h} \\
0 / 1.2 \mathrm{E}+6 \mathrm{~h} \\
{[3]}\end{array}$ & & & & $\begin{array}{l}1.4 \mathrm{E}-7 / \mathrm{h}(10) \\
{[2]}\end{array}$ \\
\hline $\begin{array}{l}\text { Soleswid-Operated } \\
\text { Fails open }\end{array}$ & & & & & $\begin{array}{l}2 / 1.6 \mathrm{E}+6 \mathrm{~d} \\
{[4]}\end{array}$ & & $\begin{array}{l}1.6 E-6 / d(10) \\
{[4]}\end{array}$ \\
\hline Fails closed & & & & & [4] & & [4] \\
\hline Fails to respond & & & & & [4] & & [4] \\
\hline \multicolumn{8}{|l|}{ Phugs } \\
\hline \multicolumn{8}{|l|}{ Leakage (external) } \\
\hline \multicolumn{8}{|l|}{ Rupture (external) } \\
\hline $\begin{array}{l}\text { Mpressor } \\
\text { Mutor-Driven } \\
\text { Fails to start }\end{array}$ & & $\begin{array}{l}5.2 / 3.2 \mathrm{E}+6 \mathrm{~h} \\
{[9]}\end{array}$ & & & $\begin{array}{l}2 / 6.4 E+4 d \\
{[5]}\end{array}$ & $\begin{array}{l}116 / 2.3 \mathrm{E}+6 \mathrm{~h} \\
{[1]}\end{array}$ & $\begin{array}{l}3.9 \mathrm{E}-5 / \mathrm{d}(10) \\
8.2 \mathrm{E}-6 / \mathrm{h}(3.6)\end{array}$ \\
\hline Fails to $\mathrm{nm}$ & & $\begin{array}{l}8.6 / 3.2 \mathrm{E}+6 \mathrm{~h} \\
{[9]}\end{array}$ & $\begin{array}{l}80 / 3.1 \mathrm{E}+4 \mathrm{~h} \\
129 / 2.0 \mathrm{E}+4 \mathrm{~h} \\
\text { [4] }\end{array}$ & & $\begin{array}{l}1 / 4.5 \mathrm{E}+4 \mathrm{~h} \\
{[5]}\end{array}$ & [1] & $\begin{array}{l}1.3 \mathrm{E}-5 / \mathrm{h}(3.6), \\
4.1 \mathrm{E}-3 \mathrm{~h}(2.0) \\
{[5]}\end{array}$ \\
\hline Overspeed & & $\begin{array}{l}1.7 / 3.2 \mathrm{E}+6 \mathrm{~h} \\
{[9]}\end{array}$ & & & & [1] & $\begin{array}{l}2.6 \mathrm{E}-6 \mathrm{~h}(5) \\
{[5]}\end{array}$ \\
\hline Fails to stop & & & & & & [1] & \\
\hline Leakage (external) & & $\begin{array}{l}0 / 3.2 \mathrm{E}+6 \mathrm{~h} \\
{[9]}\end{array}$ & & & & & $\begin{array}{l}7.2 \mathrm{E}-7 / \mathrm{h}(10) \\
{[5]}\end{array}$ \\
\hline Rupture (external) & & [9] & & & & & \\
\hline $\begin{array}{l}\text { Piping/Hose/Jumper/Tube } \\
\text { Piping } \\
\text { Leakage (external) } \\
\end{array}$ & & & & & & & \\
\hline
\end{tabular}


WSRC-TR-93-262

\begin{tabular}{|c|c|c|c|c|c|c|c|}
\hline \multirow[t]{2}{*}{ System/Component/Failure Mode } & \multicolumn{7}{|c|}{ Failure Data (Category 2 Sources) } \\
\hline & NUCLARR2 & NPKD-3 & $\overline{\text { OREDA }}$ & WIN-330 & TRITIUM & LNG1 & $\begin{array}{l}\text { Aggregated } \\
\text { Results } \\
\end{array}$ \\
\hline \multicolumn{8}{|l|}{ Ruprure (external) } \\
\hline \multicolumn{8}{|l|}{ Plugs } \\
\hline \multicolumn{8}{|l|}{$\begin{array}{l}\text { Hose } \\
\text { Leakage (external) }\end{array}$} \\
\hline \multicolumn{8}{|l|}{ Rupture (external) } \\
\hline \multicolumn{8}{|l|}{ Plugs } \\
\hline \multicolumn{8}{|l|}{$\begin{array}{l}\text { Jumper } \\
\text { Leakage (extemal) }\end{array}$} \\
\hline \multicolumn{8}{|l|}{ Ruppure (external) } \\
\hline \multicolumn{8}{|l|}{ Plugs } \\
\hline \multicolumn{8}{|l|}{$\begin{array}{l}\text { Tube } \\
\text { Leakage (exterma) }\end{array}$} \\
\hline \multicolumn{8}{|l|}{ Rupure (external) } \\
\hline \multicolumn{8}{|l|}{ Plugs } \\
\hline $\begin{array}{l}\text { Vessel } \\
\text { Tank (Pressurized) } \\
\text { Leakage (externa) }\end{array}$ & & $\begin{array}{l}0.3 / 2.8 \mathrm{E}+6 \mathrm{~h} \\
{[10]}\end{array}$ & & & $\begin{array}{l}0 / 4.8 \mathrm{E}+4 \mathrm{~h}, \\
0 / 9.5 \mathrm{E}+4 \mathrm{~h}, \\
0 / 4.8 \mathrm{E}+4 \mathrm{~h} \\
{[6]}\end{array}$ & & $\begin{array}{l}2.3 \mathrm{E}-7 / \mathrm{h}(10) \\
{[6]}\end{array}$ \\
\hline Rupture (external) & & $\begin{array}{l}0.1 / 2.8 \mathrm{E}+6 \mathrm{~h} \\
{[10]}\end{array}$ & & & [6] & & $\begin{array}{l}7.5 \mathrm{E}-8 / \mathrm{h}(10) \\
{[6]}\end{array}$ \\
\hline \multicolumn{8}{|l|}{$\begin{array}{l}\text { Cylinder (Pressurized) } \\
\text { Leakage (external) }\end{array}$} \\
\hline \multicolumn{8}{|l|}{ Rupture (external) } \\
\hline \multicolumn{8}{|l|}{$\begin{array}{l}\text { Flange/Gasket } \\
\text { Leakage (external) }\end{array}$} \\
\hline \multicolumn{8}{|l|}{ Rupture (external) } \\
\hline \multicolumn{8}{|l|}{$\begin{array}{l}\text { Heat Exchanger } \\
\text { Shell/Tube } \\
\text { Fouling (tubes) }\end{array}$} \\
\hline \multicolumn{8}{|l|}{ Plugs (wbes) } \\
\hline Leakage (ubbes) & & & $\begin{array}{l}25 / 4.6 \mathrm{E}+5 \mathrm{~h}, \\
1 / 1.3 \mathrm{E}+5 \mathrm{~h} \\
{[5]}\end{array}$ & & & & $4.4 \mathrm{E}-5 / \mathrm{h}(1.8)$ \\
\hline
\end{tabular}


WSRC-TR-93-262

\begin{tabular}{|c|c|c|c|c|c|c|c|}
\hline \multirow[t]{2}{*}{ System/Componen/Failure Mode } & \multicolumn{7}{|c|}{ Failure Data (Category 2 Sources) ${ }^{\mathrm{a}}$} \\
\hline & NUCLARR2 & NPRD-3 & OREDA & WIN-330 & TRITIUM & LNG1 & $\begin{array}{c}\text { Aggregated } \\
\text { Results }^{\mathrm{b}} \\
\end{array}$ \\
\hline Rupture (tubes) & & & $\begin{array}{l}0 / 4.6 \mathrm{E}+5 \mathrm{~h}, \\
0 / 1.3 \mathrm{E}+5 \mathrm{~h} \\
{[5]}\end{array}$ & & & & $\begin{array}{l}8.5 \mathrm{E}-7 / \mathrm{h}(10) \\
{[2]}\end{array}$ \\
\hline \multicolumn{8}{|l|}{ Leakage (s 1 ill) } \\
\hline \multicolumn{8}{|l|}{ Ruprure (shcll) } \\
\hline $\begin{array}{l}\text { Heater (Electrical) } \\
\text { Fails to heat }\end{array}$ & & & & & $\begin{array}{l}0 / 4.8 \mathrm{E}+4 \mathrm{~h} \\
{[7]}\end{array}$ & & $\begin{array}{l}1.0 \mathrm{E}-5 / \mathrm{h}(10) \\
{[2]}\end{array}$ \\
\hline \multicolumn{8}{|l|}{ Overheats } \\
\hline \multicolumn{8}{|l|}{ Leakage (external) } \\
\hline \multicolumn{8}{|l|}{ Rupture (external) } \\
\hline $\begin{array}{l}\text { Vaporizer } \\
\text { Falure }\end{array}$ & & & & & & $\begin{array}{l}26 / 1.9 \mathrm{E}+5 \mathrm{~h} \\
{[2]}\end{array}$ & $1.4 \mathrm{E}-4 / \mathrm{h}(10)$ \\
\hline $\begin{array}{l}\text { Air Dryer } \\
\text { Fonture }\end{array}$ & & & $\begin{array}{l}1 / 1.2 \mathrm{E}+5 \mathrm{~h} \\
{[6]}\end{array}$ & & $\begin{array}{l}0 / 4.8 E+4 h \\
{[8]}\end{array}$ & & $8.9 \mathrm{E}-6 / \mathrm{h}(\mathrm{i} 0)$ \\
\hline $\begin{array}{l}\text { Filter } \\
\text { Phugs }\end{array}$ & & & & $\begin{array}{l}61 / 4.2 \mathrm{E}+6 \mathrm{~h} \\
{[1]}\end{array}$ & $\begin{array}{l}0 / 1.9 \mathrm{E}+5 \mathrm{~h} \\
{[9]}\end{array}$ & & $\begin{array}{l}1.4 \mathrm{E}-5 / \mathrm{h}(1.3) \\
{[4]}\end{array}$ \\
\hline Leakage (intemal) & & & & [1] & [9] & & [4] \\
\hline Ruphure (iniernal) & & & & [1] & [9] & & [4] \\
\hline $\begin{array}{c}\text { Orifice } \\
\text { Plugs }\end{array}$ & & & & & $\begin{array}{l}0 / 2.4 \mathrm{E}+5 \mathrm{~h} \\
{[10]}\end{array}$ & & $\begin{array}{l}2.1 \mathrm{E}-6 / \mathrm{h}(10) \\
{[2]}\end{array}$ \\
\hline
\end{tabular}

Table 4c. Category 2 data for compressed gas system.

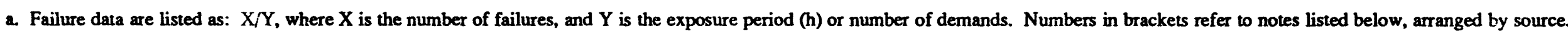

b. Aggregated results are mean and error factor (in parentheses). The error factor is the 95 th percentile/50th percentile.

Notes

NUCLARR2 - S. A. Eide et al., Generic Component Failure Data Base for Light Water and Liquid Sodium Reactor PRAs, Idaho National Engineering Laboratory, EGG-SSRE-8875, February 1990. Also, S. A. Eide et al., Component External Leakage and Rupture Frequency Estimates, Idaho National Engineering Laboratory, EGG-SSRE-9639, November 1991.

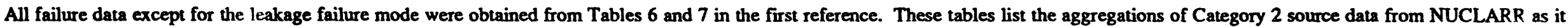

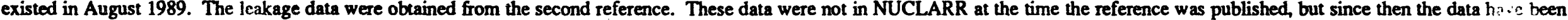
entered into NUCLARR. 
NPRD-3 - M. J. Rossi, Nonelectronic Parts Reliability Data, Reliability Analysis Center, Rome Air Development Center, NPRD-3, 1985.

all frihre data apply to a ground fixed (GF) environment, unless otherwise indicated. Commercial (C), military (M), and unknown source (?) data were used if available. Failure mode

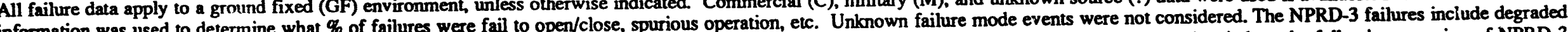

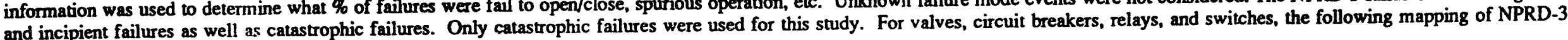
and incipient failures as well as catastrophic failures. Onil

Fail to open/close/operate - binding, breach (not for valves), contaminated, cracked/fractured, no movement, no operation, seized, stuck open, and stuck closed

Spurious operation - arcing, burned, collapsed, displaced, false response, improper flow, intermittent, opened, overheated, and shorted

Plugs - none identified

Leakage (internal) - none identified except for leaking (one-half of events applied to intemal leakage for check and relief valves)

Leakage (extemal) - leaking

Rupture (external) - breach (valves only).

For pumps, fans, and diesel generators, the following mapping was used:

Fail to start - contaminated, crackedfrectured, no movement, no operation

Fail to run - binding, broken, displaced, false response, improper flow, intermittent, overheated, seized, shorted, stuck closed, stuck open

Overspeed (subset of fail to nun - improper flow (one-half of events used), stuck open

Fail to stop - none identified

Leakage (external) - leaking

Table 4c. Category 2 data for compressed gas system.

Rupture (external) - breach (not for generator)

For vessels, piping, hoses, and jumpers, the following mapping was used:

Leakage (external) - cracked/fractured, leaking

Rupture (external) - broken, breach.

For filters, the following mapping was used:

Plug - improper flow (one-half of events used)

Leakage (internal) - improper flow (one-half of events used) 
Rupture (internal) - broken, cracked/fractured, ruptured.

For heaters, the following mapping was used:

Fail to heat - cracked/fractured, no operation, opened

Overheat - shorted.

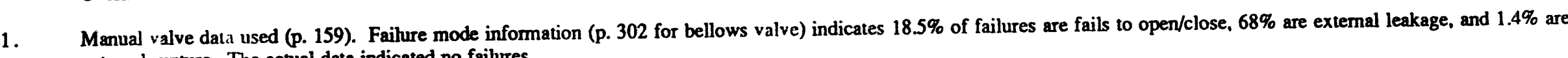
external rupture. The actual data indicated no failures.

2. Check valve data used (p. 158). Failure mode information (p. 303) indicates $9.2 \%$ of failures are fails to open and $20 \%$ are fails to close.

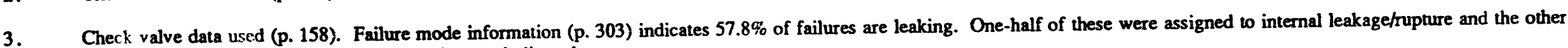
half axternal leakage. Internal leakage size not indicated.

4. Checs valve data used (p. 158). Failure mode information (p. 303) indicates $0 \%$ of failures are external rupture.

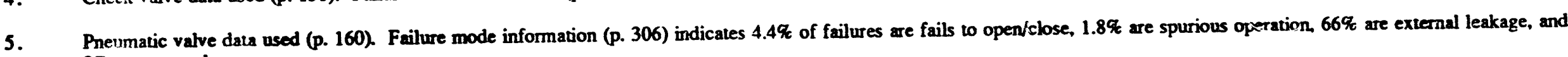
O\& are external rupture.

6. Solenoid valve data used (p. 161). See Note 5 for failure mode information.

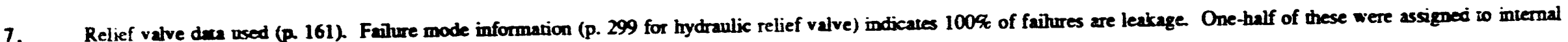
leakagefrupture and the other half to extemal leakage.

8. Relief valve data used (p. 161). Failure mode information (p. 299 for hydraulic relief valve) indicates $0 \%$ of failures are external rupture.

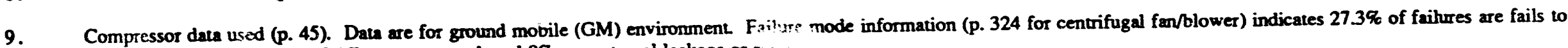
start, $455 \%$ are fails $10 \mathrm{run}, 9.1 \%$ are overspeed, and $0 \%$ are external leakage or $r$ :

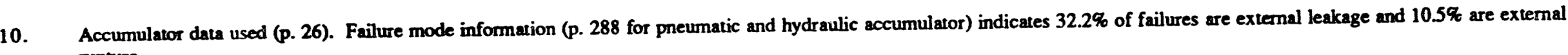
rupture.

Table 4c. Category 2 data for compressed gas system.

OREDA - Offshore Reliability Data Handbook, Offshore Reliability Data (OREDA), Norway, 1984.

All failure information was taken from the critical failure mode categciy, unless otherwise indicated.

1. Check valve (hydrocarbon gas) data used (p. 163). Plugged data used for fails to open. Significant internal leakage data used for internal rupture.

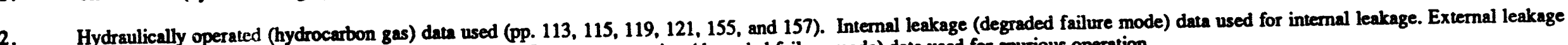
(degraded failure mode) data used for external leakage. Improper operation (degraded failure mode) data used for spurious operation.

Hydraulically operated and pneumatically operated (hydrocarbon gas) control valve data used (pp. 165 and 169). Internal leakage (degraded failure mode) data used for fails open. Failed to operate data used for fails to respond. External leakage (degraded failure mode) data used for external leakage. 
4. Centrifugal and reciprocating ( $>1500$ psig) compressor data used (pp. 229 and 231 ). No gas flow data used for fails to run.

5. Gas/water (p. 197), gas/freon (p. 201), and air/water (p. 209) tube/shell heat exchanger data used. Leakage (degraded failure mode) data used for tube failure.

6. Electric heater (non hydrocarbon service) data used (p. 219).

WIN-330 - J. N. Wilkinson et al., Idahe Chemical Processine Plant Failure Rate Database, Idaho National Engineering Laboratory, WIN-330, October 1991.

All failure information was taken from Appendix C, p. 32.

1. Filter (offgas) data used. Failure modes not indicated.

TRITIUM - L. C. Cadwallader and M. A. Stolpe Gavett. Tritium Waste Treatment System Component Failure Data Analysis from June 18. 1984 to December 31.1989 Idaho National

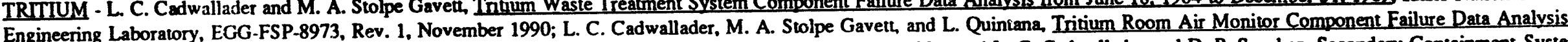

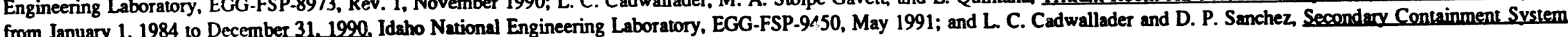
Compent Faihure Data Analysis from 1984 io 1991 Idaho National Engineering Laboratory, EGG-FSP-10323, August 1992

1. Manual valve data used (Table A-1, first reference). Faihure modes not indicated.

2. Mowr-operated valve data used (Table A-1. fint reference). Faihure modes (except for leak) not indicared

3. Pressure control valve data used (Table A-1, first reference and p. 23, third reference). Faihure modes (other than leak) not indicated.

4. Solenoid control valve data used (p. 27, third reference). Failure modes (other than leak) not indicated.

5. Compressor data used (Table A-1, third reference).

6. Tritium waste gas tank data used (Table A-1, first reference).

7. Electrical heater data used (Table A-1, first reference). Failure modes (except for leak) not indicated.

8. Moisture collector data used (Table A-1, first reference). Failure modes not indicated.

Table 4c. Category 2 data for compressed gas system.

9. Filter data used (Table A-1, first reference). Failure modes not indicated.

10. Orifice data used (Table A-1, first reference).

LNG1 - D. W. Johnson and J. R. Welker, Development of an Improved LNG Plant Failure Rate Data Base, Gas Research Institute, PB82-153503, September 1981.

1. Compressor system data used (p. 9). Failure modes not indicated.

2. Vaporizer data used (p. 9). 


\section{Aggreqated Results}

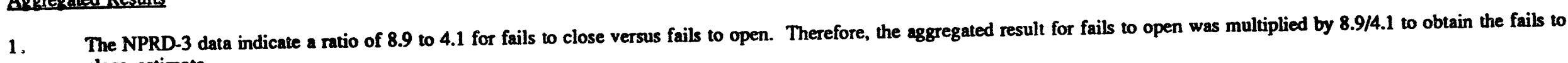
close estimate.

2. Faihure rate based on zero failures and may be conservatively high.

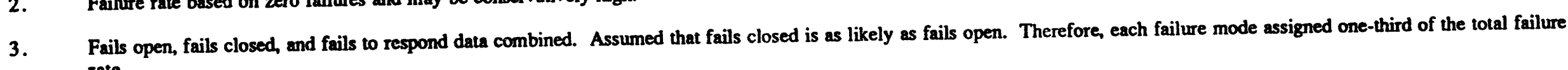
rate.

4. Failure modes not indicated.

5. All failure modes combined. OREDA data treated separately. NPRD-3 data used to partition among failure modes.

6. Both failure modes combined. NPRD-3 data used to partition between failure modes. 
WSRC-TR-93-262

\begin{tabular}{|c|c|c|c|c|c|c|c|}
\hline \multirow[t]{2}{*}{ System/Component/Failure Mode } & \multicolumn{7}{|c|}{ Failure Data (Category 2 Sources) } \\
\hline & NUCLARR2 & NPRD-3 & OREDA & WIN-330 & TRTIUM & LNG1 & $\begin{array}{l}\text { Aggregated } \\
\text { Results }\end{array}$ \\
\hline \multicolumn{8}{|l|}{$\begin{array}{l}\text { HVAC/Exhaust } \\
\text { Damper (Standby or Safery) } \\
\text { Manual } \\
\text { Fails to open/close } \\
\end{array}$} \\
\hline \multicolumn{8}{|l|}{ Plugs } \\
\hline \multicolumn{8}{|l|}{ Leakage (intemal) } \\
\hline \multicolumn{8}{|l|}{ Rupture (internal) } \\
\hline \multicolumn{8}{|l|}{ Leakage (external) } \\
\hline \multicolumn{8}{|l|}{ Rupture (external) } \\
\hline \multicolumn{8}{|l|}{$\begin{array}{l}\text { Motor-Opermed } \\
\text { Fails to open/close }\end{array}$} \\
\hline \multicolumn{8}{|l|}{ Spurious operation } \\
\hline \multicolumn{8}{|l|}{ Proges } \\
\hline \multicolumn{8}{|l|}{ Lestage (incomin) } \\
\hline \multicolumn{8}{|l|}{ Rupure (miems) } \\
\hline \multicolumn{8}{|l|}{ Leakage (extermal) } \\
\hline \multicolumn{8}{|l|}{ Rupture (external) } \\
\hline \multicolumn{8}{|l|}{$\begin{array}{l}\text { Air-Operated } \\
\text { Fails to open/close }\end{array}$} \\
\hline \multicolumn{8}{|l|}{ Spurious operation } \\
\hline \multicolumn{8}{|l|}{ Plugs } \\
\hline \multicolumn{8}{|l|}{ Leakage (internal) } \\
\hline \multicolumn{8}{|l|}{ Rupture (intemal) } \\
\hline \multicolumn{8}{|l|}{ Leakage (external) } \\
\hline \multicolumn{8}{|l|}{ Rupture (external) } \\
\hline \multicolumn{8}{|l|}{$\begin{array}{c}\text { Damper (Control) } \\
\text { Motor-Operated } \\
\text { Fails open } \\
\end{array}$} \\
\hline \multicolumn{8}{|l|}{ Fails closed } \\
\hline \multicolumn{8}{|l|}{ Fails to respond } \\
\hline Phugs & & & & & & & \\
\hline
\end{tabular}


WSRC-TR-93-262

\begin{tabular}{|c|c|c|c|c|c|c|c|}
\hline \multirow[t]{2}{*}{ System/Component/Failure Mode } & \multicolumn{7}{|c|}{ Failure Data (Category 2 Sources) } \\
\hline & NUCLARR2 & NPRD-3 & OREDA & WIN-330 & TRITIUM & LNG1 & $\begin{array}{c}\text { Aggregated } \\
\text { Results }^{\mathbf{b}} \\
\end{array}$ \\
\hline \multicolumn{8}{|l|}{ Leakage (cxternal) } \\
\hline \multicolumn{8}{|l|}{ Rupture (external) } \\
\hline $\begin{array}{r}\text { Air-Operated } \\
\text { Fails open } \\
\end{array}$ & & & $\begin{array}{l}48 / 6.1 \mathrm{E}+6 \mathrm{~h} \\
{[1]}\end{array}$ & & & & $\begin{array}{l}8.0 \mathrm{E}-6 / \mathrm{h}(10) \\
{[1]}\end{array}$ \\
\hline Fails closed & & & [1] & & & & [1] \\
\hline Fails to respond & & & $\begin{array}{l}5 / 6.1 \mathrm{E}+6 \mathrm{~h} \\
\text { [1] }\end{array}$ & & & & $9.0 \mathrm{E}-7 / \mathrm{h}(10)$ \\
\hline \multicolumn{8}{|l|}{ Plugs } \\
\hline Leakage (external) & & & $\begin{array}{l}2 / 6.1 \mathrm{E}+6 \mathrm{~h} \\
{[1]}\end{array}$ & & & & $4.1 \mathrm{E}-7 / \mathrm{h}(10)$ \\
\hline Rupare (extermal) & & & $\begin{array}{l}1 / 6.1 \mathrm{E}+6 \mathrm{~h} \\
{[1]}\end{array}$ & & & & $2.5 \mathrm{E}-7 / \mathrm{h}(10)$ \\
\hline $\begin{array}{l}\text { Fan/Blowa } \\
\text { Motor-Diven } \\
\text { Fails to star }\end{array}$ & & $\begin{array}{l}2.216 .7 \mathrm{E}+5 \mathrm{~h} . \\
4.6 / 6.1 \mathrm{E}+6 \mathrm{~h} . \\
11.2 / 2.3 \mathrm{E}+6 \mathrm{~h} \\
11]\end{array}$ & $\begin{array}{l}24 / 3.5 \mathrm{E}-6 \mathrm{~h} \\
{[2]}\end{array}$ & & & & $3.3 \mathrm{E}-6 / \mathrm{h}(2.9)$ \\
\hline Fails to run & & $\begin{array}{l}3.6 / 6.7 \mathrm{E}+5 \mathrm{~h}, \\
7.7 / 6.1 \mathrm{E}+6 \mathrm{~h} \\
18.6 / 2.3 \mathrm{E}+6 \mathrm{~h} \\
{[1]}\end{array}$ & $\begin{array}{l}33 / 3.5 \mathrm{E}+6 \mathrm{~h} \\
{[2]}\end{array}$ & & & & $5.0 \mathrm{E}-6 / \mathrm{h}(2.8)$ \\
\hline Overspeed & & $\begin{array}{l}0.7 / 6.7 \mathrm{E}+5 \mathrm{~h}, \\
1.5 / 6.1 \mathrm{E}+6 \mathrm{~h}, \\
3.7 / 2.3 \mathrm{E}+6 \mathrm{~h} \\
{[1]}\end{array}$ & & & & & $6.5 \mathrm{E}-7 / \mathrm{h}(1.9)$ \\
\hline Fails to stop & & & $\begin{array}{l}12 / 3.5 \mathrm{E}+6 \mathrm{~h} \\
{[2]}\end{array}$ & & & & $3.6 \mathrm{E}-6 / \mathrm{h}(10)$ \\
\hline Leakage (external) & & $\begin{array}{l}0 / 6.7 \mathrm{E}+5 \mathrm{~h}, \\
0 / 6.1 \mathrm{E}+6 \mathrm{~h}, \\
0 / 2.3 \mathrm{E}+6 \mathrm{~h} \\
{[1]} \\
\end{array}$ & & & & & $\begin{array}{l}5.5 \mathrm{E}-8 / \mathrm{h}(10) \\
{[1,2]}\end{array}$ \\
\hline Rupture (external) & & [1] & & & & & {$[1,2]$} \\
\hline \multicolumn{8}{|l|}{$\begin{array}{c}\text { Diesel-Driven } \\
\text { Fails to start }\end{array}$} \\
\hline \multicolumn{8}{|l|}{ Fails to run } \\
\hline Overspeed & & & & & & & \\
\hline
\end{tabular}


WSRC-TR-93-262

\begin{tabular}{|c|c|c|c|c|c|c|c|}
\hline \multirow[t]{2}{*}{ System/Component/Failure Mode } & \multicolumn{7}{|c|}{ Failure Data (Category 2 Sources) $)^{\mathrm{a}}$} \\
\hline & NUCLARR2 & NPRD-3 & OREDA & WIN-330 & TRITIUM & LNG1 & $\begin{array}{l}\text { Aggregated } \\
\text { Results }\end{array}$ \\
\hline \multicolumn{8}{|l|}{ Fails to stop } \\
\hline \multicolumn{8}{|l|}{ Leakage (external) } \\
\hline \multicolumn{8}{|l|}{ Rupture (external) } \\
\hline \multicolumn{8}{|l|}{$\begin{array}{l}\text { Ducting } \\
\quad \text { Leakage (external) }\end{array}$} \\
\hline \multicolumn{8}{|l|}{ Rupcure (external) } \\
\hline \multicolumn{8}{|l|}{ Plugs } \\
\hline \multicolumn{8}{|l|}{$\begin{array}{l}\text { Heat Exchanger } \\
\text { Air Conditioning Unit/ } \\
\text { Chiller } \\
\text { Fails to stan } \\
\end{array}$} \\
\hline \multicolumn{8}{|l|}{ Faiks 00} \\
\hline \multicolumn{8}{|l|}{$\begin{array}{l}\text { Fan Cooker Lini } \\
\text { Fails to stan }\end{array}$} \\
\hline Faiks to run & & & $\begin{array}{l}0 / 1.4 \mathrm{E}+5 \mathrm{~h} \\
{[3]}\end{array}$ & & & & $\begin{array}{l}3.6 \mathrm{E}-6 / \mathrm{h}(10) \\
{[2]}\end{array}$ \\
\hline $\begin{array}{l}\text { Heater (Electrical) } \\
\text { Fails to heat }\end{array}$ & & $\begin{array}{l}1.2 / 4.2 \mathrm{E}+4 \mathrm{~h}, \\
8.0 / 1.1 \mathrm{E}+7 \mathrm{~h} \\
{[2]}\end{array}$ & & & & & $8.4 \mathrm{E}-7 / \mathrm{h}(2.6)$ \\
\hline Overheats & & $\begin{array}{l}0.2 / 4.2 \mathrm{E}+4 \mathrm{~h}, \\
1.0 / 1.1 \mathrm{E}+7 \mathrm{~h} \\
{[2]}\end{array}$ & & & & & $1.6 \mathrm{E}-7 / \mathrm{h}(10)$ \\
\hline $\begin{array}{l}\text { Heater (Gas) } \\
\text { Fails to heat }\end{array}$ & & & $\begin{array}{l}72 / 8.8 \mathrm{E}+4 \mathrm{~h} \\
\text { [4] }\end{array}$ & & & & $8.2 \mathrm{E}-4 / \mathrm{h}(10)$ \\
\hline Overheats & & & $\begin{array}{l}1 / 8.8 \mathrm{E}+4 \mathrm{~h} \\
{[4]}\end{array}$ & & & & $1.7 \mathrm{E}-5 / \mathrm{h}(10)$ \\
\hline $\begin{array}{l}\text { Filter } \\
\text { Normal } \\
\text { Plugs }\end{array}$ & & $\begin{array}{l}0 / 5.8 \mathrm{E}+6 \mathrm{~h} \\
{[3]}\end{array}$ & $\begin{array}{l}5 / 5.0 \mathrm{E}+5 \mathrm{~h} \\
{[5]}\end{array}$ & $\begin{array}{l}34 / 6.1 \mathrm{E}+6 \mathrm{~h} \\
{[1]}\end{array}$ & & & $\begin{array}{l}1.4 \mathrm{E}-6 \mathrm{~h}(5) \\
{[3]}\end{array}$ \\
\hline Leakage (intemal) & & $\begin{array}{l}5.2 / 5.8 \mathrm{E}+6 \mathrm{~h} \\
{[3]}\end{array}$ & $\begin{array}{l}1 / 5.0 \mathrm{E}+5 \mathrm{~h} \\
{[5]}\end{array}$ & [1] & & & $\begin{array}{l}1.8 \mathrm{E}-6 / \mathrm{h}(5) \\
{[3]}\end{array}$ \\
\hline Rupture (internal) & & $\begin{array}{l}1.5 / 5.8 \mathrm{E}+6 \mathrm{~h} \\
{[3]}\end{array}$ & $\begin{array}{l}1 / 5.0 \mathrm{E}+5 \mathrm{~h} \\
{[5]}\end{array}$ & [1] & & & $\begin{array}{l}7.0 \mathrm{E}-7 \mathrm{~h}(5) \\
{[3]}\end{array}$ \\
\hline $\begin{array}{l}\text { Low-Efficiency } \\
\text { Plugs }\end{array}$ & & & & & & & \\
\hline
\end{tabular}




\begin{tabular}{|c|c|c|c|c|c|c|c|}
\hline \multirow[t]{2}{*}{ System/Component/Failure Mode } & \multicolumn{7}{|c|}{ Failure Data (Category 2 Sources) ${ }^{\mathrm{a}}$} \\
\hline & NUCLARR2 & NPRD-3 & OREDA & WIN-330 & TRITIUM & LNG1 & $\begin{array}{l}\text { Aggregated } \\
\text { Results }{ }^{b}\end{array}$ \\
\hline \multicolumn{8}{|l|}{ Leakage (intemal) } \\
\hline \multicolumn{8}{|l|}{ Rupture (intemal) } \\
\hline \multicolumn{8}{|l|}{$\begin{array}{l}\text { HEPA } \\
\text { Phugs }\end{array}$} \\
\hline \multicolumn{8}{|l|}{$\begin{array}{l}\text { Plugs } \\
\text { Leakage (internal) }\end{array}$} \\
\hline \multicolumn{8}{|l|}{ Rupture (intemal) } \\
\hline \multicolumn{8}{|l|}{$\begin{array}{l}\text { Sand } \\
\text { Phugs } \\
\end{array}$} \\
\hline \multicolumn{8}{|l|}{$\begin{array}{l}\text { Phugs } \\
\text { Leakage (internal) }\end{array}$} \\
\hline \multicolumn{8}{|l|}{ Ruppure (intermal) } \\
\hline \multicolumn{8}{|l|}{$\begin{array}{l}\text { Baphouse } \\
\text { Ph:-: }\end{array}$} \\
\hline \multicolumn{8}{|l|}{ Ph $=\frac{}{\text { se (iniemal) }}$} \\
\hline \multicolumn{8}{|l|}{$\bar{R} \cdots+\ldots$ (internal) } \\
\hline \multicolumn{8}{|l|}{$\begin{array}{l}\text { Misce: : :ius } \\
\text { Mist Eliminator } \\
\text { Failure }\end{array}$} \\
\hline $\begin{array}{l}\text { Sirubber } \\
\text { Failure }\end{array}$ & & & $\begin{array}{l}1 / 4.7 \mathrm{E}+6 \mathrm{~h} \\
16]\end{array}$ & & & & $3.2 \mathrm{E}-7 / \mathrm{h}(10)$ \\
\hline $\begin{array}{l}\text { Precipitator } \\
\text { Failure }\end{array}$ & & & & & & & \\
\hline
\end{tabular}

Table 4d. Category 2 data for HVAC/exhaust system.

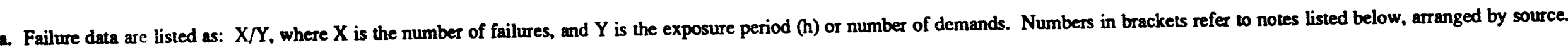
b. Aggregated results are mean and error factor (in parentheses). The error factor is the 95 th percentile/50th percentile. 
Notes

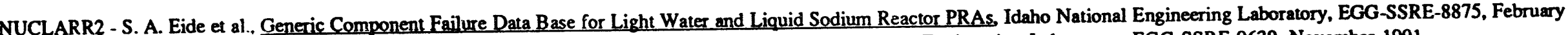
1990. Also, S. A. Eide et al. Component External Leakage and Rupture Erequency Estimates, Idaho National Engineering Laboratory, EGG-SSRE-9639, November 1991.

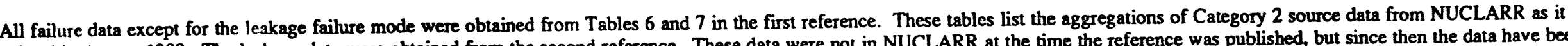

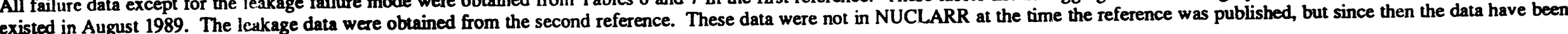
entered into NUCLARR.

NPRD-3 - M. J. Rossi, Nonelecironic Parts Reliability Data, Reliability Analysis Center, Rome Air Development Center, NPRD-3, 1985.

All failure data apply to a ground fixed (GF) environment, unless otherwise indicated. Commercial (C), military (M), and unknown source (?) data were used if available. Failure mode

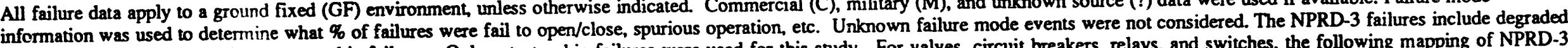

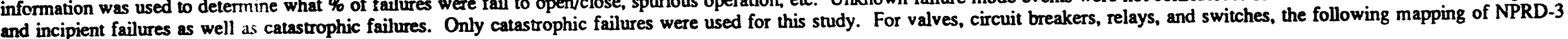
failure modes into the failure modes of interest was used:

Fail to open/close/operate - binding. breach (not for valves), contaminated, cracked/fractured, no movement, no operation, seized, stuck open, and stuck closed

Spurious operation - arcing, burned, collapsed, displaced, false response, improper flow, intermittent, opened, overheated, and shorted

Plugs - none identified

Leakage (intemal) - none identified except for leaking (one-half of events applied to internal leakage for checix and relief valves)

Leakage (extemal) - leaking

Rupture (external) - breach (valves only).

For pumps, fans, and diesel generators, the following mapping was used:

Fail to start - contaminated, cracked/fractured, no movement, no operation

Fail to run - binding, broken, displeced, false response, improper flow, intermittent, overheated, seized, shorted, stuck closed, stuck open

Overspeed (subset of fail to run) - improper flow (one-half of events used), stuck open

Fail to stop - none identified

Leakage (extemal) - leaking

Table 4d. Category 2 data for HVAC/exhaust system.

Rupture (external) - breach (not for generator)

For vessels, piping, hoses, and jumpers, the following mapping was used:

Leakage (external) - cracked/fractured, leaking 
Rupture (external) - Broken, breach.

For filters, the following mapping was used:

Plug - improper flow (one-half of events used)

Leakage (internal) - improper flow (one-half of events used)

Rupture (internal) - broken, cracked/fractured, ruptured.

For heaters, the following mapping was used:

Fail to heat - cracked/fractured, no operation, opened

Overheat - shorted.

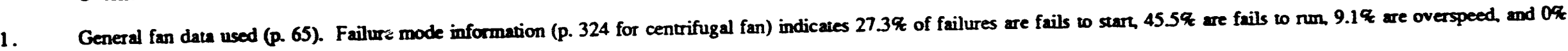
are extemal leakage or rupure.

Duct hearer (p. 79) and electric space beater (p. 81) dala used respectively. Failure mode information (p. 287 for resistive electrical bearer) indicates 6154 of failures are fails 6 bea and 7.79 are oreaters.

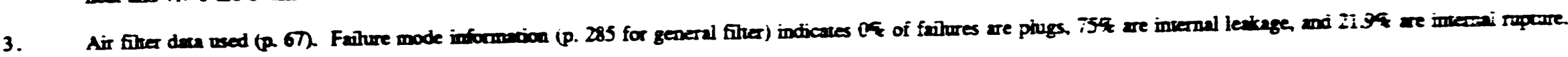

OREDA - Offshore Relinbility Dun Handbook, Offsbore Reliability Data (OREDA), Norway, 1984.

All failure information was taken from the critical failure mode category, unless otherwise indicated

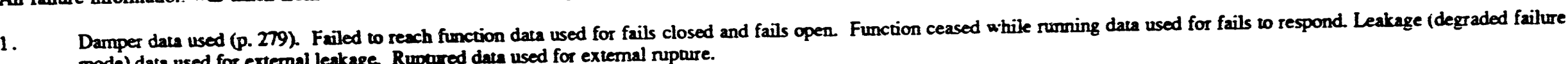
mode) data used for extemal leakage. Ruptured data used for extemal nupure.

Fan uas used (p. 277). Failed to operate upon command and failed to function when signaled data used for fails to start. Failed while running data used for fails to run. Functioned without signal data considered similar to fails to stop.

3. Cooler (nun hydrocarbon service) data used (p. 221).

4. Gas/diesel fired boilex data used (p. 283).

Table 4d. Category 2 data for HVAC/exhaust system.

5. Filter data used (p. 281). Clogged data used for plugs. Internal leakage (degraded faikure mode) data used for intemal leakage. Ruptured data used for internal rupture.

6. Scrubber (removal of oil/condensate from hydrocarbon gas) data used (p. 147). Plugged or clogged data used for failure.

WIN-330 - J. N. Wilkinson et al., Idaho Chemical Processing Plant Failure Rate Database, Idaho National Engineering Laboratory, WIN-330, October 1991. 
All failure information was taken from Appendix C, p. 32.

1.

Filter (other) data used. Failure modes not indicated.

TRITIUM - L. C. Cadwallader and M. A. Stolpe Gavett. Tritium Waste Treatment System Component Failure Data Analysis from June 18. 1984 to December 31. 1989, Idaho National

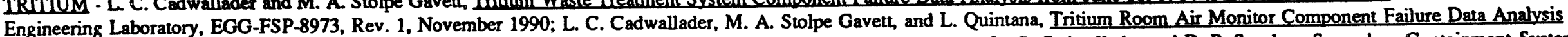

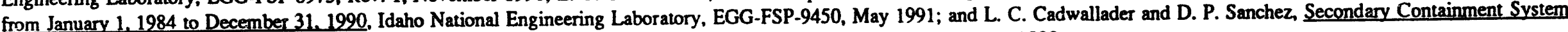
Component Failure Data Analysis from 1984 to 1991, Idaho National Engineering Laboratory, EGG-FSP-10323, August 1992.

LNG1 - D. W. Johnson and J. R. Welker, Development of an Improved LNG Plant Failure Rate Dais Base, Gas Research Institute, PB82-153503, September 1981.

Aggregated Results

1. Faiture modes not indicared.

2. Faiture rase besed on zero falures and may be conseriativety high

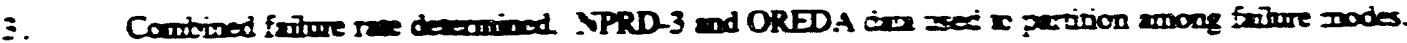


WSRC-TR-93-262

\begin{tabular}{|c|c|c|c|c|c|c|c|}
\hline \multirow[t]{2}{*}{ System/Component/Failure Mode } & \multicolumn{7}{|c|}{ Failure Data (Category 2 Sources) } \\
\hline & NUCLARR2 & NPRD-3 & OREDA & WIN-330 & TRITUM & LNG1 & $\begin{array}{c}\text { Aggregated } \\
\text { Results }^{\mathbf{b}}\end{array}$ \\
\hline $\begin{array}{l}\text { Electrical Distribution } \\
\text { Generator } \\
\text { Diesel-Driven } \\
\text { Fails to }\end{array}$ & $\begin{array}{l}83 / 2.2 E+4 d \\
{[1]}\end{array}$ & $\begin{array}{l}149 / 5.9 \mathrm{E}+5 \mathrm{~h} \\
{[1]}\end{array}$ & $\begin{array}{l}18 / 5.0 \mathrm{E}+5 \mathrm{~h}, \\
6 / 7.6 \mathrm{E}+2 \mathrm{~d} \\
{[1]}\end{array}$ & & & & $\begin{array}{l}3.9 \mathrm{E}-3 / \mathrm{d}(3.0) \\
4.1 \mathrm{E}-5 / \mathrm{h}(3.0) \\
{[1]}\end{array}$ \\
\hline Fails to run & $\begin{array}{l}140 / 1.4 E+4 d \\
{[2]}\end{array}$ & [1] & $\begin{array}{l}82 / 2.2 \mathrm{E}+5 \mathrm{~h} \\
1 / 1.3 \mathrm{E}+4 \mathrm{~h} \\
11]\end{array}$ & & & & $\begin{array}{l}9.7 \mathrm{E}-3 / \mathrm{d}(3.1) \\
1.9 \mathrm{E}-4 / \mathrm{h}(1.2) \\
{[1]}\end{array}$ \\
\hline $\begin{array}{l}\text { Motor-Driven (ac to dc) } \\
\text { Fails to start }\end{array}$ & & $\begin{array}{l}4 / 3.2 \mathrm{E}+5 \mathrm{~h} \\
{[2]}\end{array}$ & & & & & $1.4 \mathrm{E}-5 / \mathrm{h}(10)$ \\
\hline Fails to run & & $2 / 3.2 \mathrm{E}+5 \mathrm{~h}$ & & & & & $7.8 \mathrm{E}-6 / \mathrm{h}(10)$ \\
\hline $\begin{array}{l}\text { Gas-Turbine-Driven } \\
\text { Fails to start }\end{array}$ & & $\begin{array}{l}5.0 / 4.0 \mathrm{E}+6 \mathrm{~h} \\
35.5 / 5.4 \mathrm{E}+5 \mathrm{~h} \\
{[3]}\end{array}$ & $\begin{array}{l}6 / 1.8 \mathrm{E}+5 \mathrm{~h} \\
51 / 3.4 \mathrm{E}+3 \mathrm{~d} \\
6 / 7.6 \mathrm{E}+1 \mathrm{~d} \\
11 / 2.6 \mathrm{E}+3 \mathrm{~d} \\
{[2]}\end{array}$ & & & & $\begin{array}{l}1.1 \mathrm{E}-2 / \mathrm{d}(3.0) \\
9.9 \mathrm{E}-6 / \mathrm{h}(8.4)\end{array}$ \\
\hline Fails to run & & $\begin{array}{l}7.6 / 4.0 \mathrm{E}+6 \mathrm{~h} \\
53.4 / 5.4 \mathrm{E}+5 \mathrm{~h} \\
{[3]}\end{array}$ & $\begin{array}{l}43 / 2.7 \mathrm{E}+5 \mathrm{~h}, \\
7 / 1.3 \mathrm{E}+4 \mathrm{~h}, \\
5 / 4.4 \mathrm{E}+4 \mathrm{~h} \\
{[2]}\end{array}$ & & & & $2.4 \mathrm{E}-5 / \mathrm{h}(8.7)$ \\
\hline \multicolumn{8}{|l|}{$\begin{array}{l}\text { Hydro-Turbine-Driven } \\
\text { Fails to start }\end{array}$} \\
\hline \multicolumn{8}{|l|}{ Fails to run } \\
\hline $\begin{array}{l}\text { Battery } \\
\text { Failure }\end{array}$ & $\begin{array}{l}6 / 1.5 E+6 h \\
{[3]}\end{array}$ & $\begin{array}{l}6.5 / 1.1 \mathrm{E}+7 \mathrm{~h} \\
{[4]}\end{array}$ & $\begin{array}{l}4 / 5.9 E+6 h \\
{[3]}\end{array}$ & & & & $6.9 \mathrm{E}-7 / \mathrm{h}(10)$ \\
\hline $\begin{array}{l}\text { Charger } \\
\text { Rectifier } \\
\text { Faihure }\end{array}$ & $\begin{array}{l}12 / 2.2 E+6 h \\
{[4]}\end{array}$ & & $\begin{array}{l}1 / 2.7 \mathrm{E}+6 \mathrm{~h} \\
35 / 5.6 \mathrm{E}+6 \mathrm{~h} \\
{[4]}\end{array}$ & & & & $4.6 \mathrm{E}-6 / \mathrm{h}(2.5)$ \\
\hline \multicolumn{8}{|l|}{$\begin{array}{l}\text { Bus } \\
\text { Metal-Enclosed } \\
\text { Faihure }\end{array}$} \\
\hline \multicolumn{8}{|l|}{$\begin{array}{l}\text { Bare } \\
\text { Faihure }\end{array}$} \\
\hline \multicolumn{8}{|l|}{$\begin{array}{l}\text { Cable/Joint/ } \\
\text { Termination/Jumper } \\
\text { Cable (Copper, 1000ft) } \\
\text { Failure }\end{array}$} \\
\hline $\begin{array}{l}\text { Joini (Copper) } \\
\text { Faihure }\end{array}$ & & & & & & & \\
\hline
\end{tabular}


WSRC-TR-93-262

Table 4e. Category 2 data for electrical distribution system.

\begin{tabular}{|c|c|c|c|c|c|c|c|}
\hline \multirow[t]{2}{*}{ System/Componen//Failure Mode } & \multicolumn{7}{|c|}{ Failure Data (Category 2 Sources) } \\
\hline & NUCLARR2 & NPRD-3 & OREDA & WIN-330 & TRTIUM & LNG1 & $\begin{array}{c}\text { Aggregated } \\
\text { Results } \\
\end{array}$ \\
\hline \multicolumn{8}{|l|}{ Termination (Copper) } \\
\hline \multicolumn{8}{|l|}{ Failure } \\
\hline \multicolumn{8}{|l|}{$\begin{array}{l}\text { Jumper (Power) } \\
\text { Failure }\end{array}$} \\
\hline $\begin{array}{l}\text { Circuit Breaker } \\
\text { General } \\
\text { Fails to open/close }\end{array}$ & $\begin{array}{l}25 / 6.3 E+4 d \\
{[5]}\end{array}$ & $\begin{array}{l}3.3 / 7.5 \mathrm{E}+5 \mathrm{~h}, \\
40.1 / 8.9 \mathrm{E}+7 \mathrm{~h} \\
{[5]}\end{array}$ & $\begin{array}{l}5 / 2.7 \mathrm{E}+7 \mathrm{~h}, \\
9 / 1.8 \mathrm{E}+6 \mathrm{~h} \\
{[5]}\end{array}$ & & & & $\begin{array}{l}4.0 \mathrm{E}-4 / \mathrm{d}(1.4) \\
4.8 \mathrm{E}-7 / \mathrm{h}(4.8)\end{array}$ \\
\hline Spurious operation & & $\begin{array}{l}0.8 / 7.5 \mathrm{E}+5 \mathrm{~h}, \\
9.4 / 8.9 \mathrm{E}+7 \mathrm{~h} \\
{[5]}\end{array}$ & $\begin{array}{l}2 / 1.2 \mathrm{E}+6 \mathrm{~h}, \\
6 / 2.7 \mathrm{E}+7 \mathrm{~h}, \\
4 / 1.8 \mathrm{E}+6 \mathrm{~h} \\
{[5]}\end{array}$ & & & & $1.9 \mathrm{E}-7 / \mathrm{h}(4.8)$ \\
\hline \multicolumn{8}{|l|}{$\begin{array}{l}\text { Reactor Trip } \\
\text { Fails to open }\end{array}$} \\
\hline \multicolumn{8}{|l|}{ Spurious operation } \\
\hline $\begin{array}{l}\text { Relay } \\
\text { Protective } \\
\quad \text { Fails to open/close } \\
\end{array}$ & & $\begin{array}{l}8.9 / 1.9 \mathrm{E}+8 \mathrm{~h}, \\
7.9 / 1.8 \mathrm{E}+8 \mathrm{~h} \\
{[6]}\end{array}$ & & & & & $4.2 \mathrm{E}-8 / \mathrm{h}(10)$ \\
\hline Spurious operation & & $\begin{array}{l}33.6 / 1.9 \mathrm{E}+8 \mathrm{~h}, \\
29.8 / 1.8 \mathrm{E}+8 \mathrm{~h} \\
{[6]}\end{array}$ & & & & & $1.7 \mathrm{E}-7 / \mathrm{h}(10)$ \\
\hline \multicolumn{8}{|l|}{$\begin{array}{l}\text { Control } \\
\text { Fails to open/close }\end{array}$} \\
\hline \multicolumn{8}{|l|}{ Spurious } \\
\hline \multicolumn{8}{|l|}{$\begin{array}{l}\text { Bistable } \\
\text { Fails to open/close }\end{array}$} \\
\hline Spurious operation & & & & & & & \\
\hline $\begin{array}{l}\text { Switch } \\
\text { Push-Button (Manual) } \\
\text { Fails to open/close } \\
\end{array}$ & & $\begin{array}{l}1588 / 7.8 \mathrm{E}+8 \mathrm{~h}, \\
0.6 / 5.6 \mathrm{E}+7 \mathrm{~h} \\
{[7]}\end{array}$ & & & & & $1.9 \mathrm{E}-6 / \mathrm{h}(10)$ \\
\hline Spurious operation & & $\begin{array}{l}1486 / 7.8 \mathrm{E}+8 \mathrm{~h}, \\
5.6 / 5.6 \mathrm{E}+7 \mathrm{~h} \\
{[7]}\end{array}$ & & & & & $1.8 \mathrm{E}-6 / \mathrm{h}(10)$ \\
\hline $\begin{array}{l}\text { Rotary (Manual) } \\
\text { Fails to open/close }\end{array}$ & & $\begin{array}{l}0.08 / 1.1 \mathrm{E}+5 \mathrm{~h}, \\
3.9 / 7.5 \mathrm{E}+7 \mathrm{~h} \\
{[8]}\end{array}$ & & & & & $6.0 \mathrm{E}-8 / \mathrm{h}(10)$ \\
\hline Spurious operation & & $\begin{array}{l}0.7 / 1.1 \mathrm{E}+5 \mathrm{~h} \\
36.6 / 7.5 \mathrm{E}+7 \mathrm{~h} \\
{[8]}\end{array}$ & & & & & $5.0 \mathrm{E}-7 / \mathrm{h}(10)$ \\
\hline
\end{tabular}


WSRC-TR-93-262

\begin{tabular}{|c|c|c|c|c|c|c|c|}
\hline \multirow[t]{2}{*}{ System/Component/Failure Mode } & \multicolumn{7}{|c|}{ Failure Data (Category 2 Sources) ${ }^{\mathrm{a}}$} \\
\hline & NUCLARR2 & NPRD-3 & OREDA & WIN-330 & TRITIUM & LNG1 & $\begin{array}{c}\text { Aggregated } \\
\text { Results }\end{array}$ \\
\hline $\begin{array}{l}\text { Key-Operated (Manual) } \\
\text { Fails to open/close }\end{array}$ & & $\begin{array}{l}0.5 / 2.6 \mathrm{E}+6 \mathrm{~h} \\
{[9]}\end{array}$ & & & & & $3.9 \mathrm{E}-7 / \mathrm{h}(10)$ \\
\hline Spurious operation & & $\begin{array}{l}4.2 / 2.6 \mathrm{E}+6 \mathrm{~h} \\
{[9]}\end{array}$ & & & & & $1.8 \mathrm{E}-6 / \mathrm{h}(10)$ \\
\hline \multicolumn{8}{|l|}{$\begin{array}{l}\text { Automatic-Transfee } \\
\text { Fails to open/close }\end{array}$} \\
\hline \multicolumn{8}{|l|}{ Spurious operation } \\
\hline \multicolumn{8}{|l|}{$\begin{array}{l}\text { Limit } \\
\text { Fails to open/close }\end{array}$} \\
\hline Spurious operation & & & & & & & \\
\hline $\begin{array}{l}\text { Fuse } \\
\text { Fail to open }\end{array}$ & & $\begin{array}{l}61.5 / 5.4 \mathrm{E}+8 \mathrm{~h} \\
{[10]}\end{array}$ & & & & & $1.2 \mathrm{E}-7 / \mathrm{h}(10)$ \\
\hline Premanure opening & & $\begin{array}{l}8.2 / 5.4 \mathrm{E}+8 \mathrm{~h} \\
{[10]}\end{array}$ & & & & & $1.6 \mathrm{E}-8 / \mathrm{h}(10)$ \\
\hline Fallure & $\begin{array}{l}90 / 3.9 \mathrm{E}+4 \mathrm{~h} \\
{[6]}\end{array}$ & & $\begin{array}{l}6 / 1.2 \mathrm{E}+6 \mathrm{~h} \\
{[6]}\end{array}$ & & & & $1.8 \mathrm{E}-5 / \mathrm{h}(6.9)$ \\
\hline $\begin{array}{l}\text { Motor } \\
\text { Fails to stant }\end{array}$ & & $\begin{array}{l}213 / 8.3 \mathrm{E}+6 \mathrm{~h}, \\
4.5 / 6.5 \mathrm{E}+6 \mathrm{~h} \\
{[11]}\end{array}$ & & & & & $1.5 \mathrm{E}-5 / \mathrm{h}(3.4)$ \\
\hline Fails to run & & $\begin{array}{l}87.1 / 8.3 \mathrm{E}+6 \mathrm{~h} . \\
1.8 / 6.5 \mathrm{E}+6 \mathrm{~h} \\
{[11]}\end{array}$ & & & & & $6.0 \mathrm{E}-6 / \mathrm{h}(3.3)$ \\
\hline $\begin{array}{l}\mathrm{DC} \\
\text { Fails to start }\end{array}$ & & $\begin{array}{l}40.5 / 1.1 \mathrm{E}+6 \mathrm{~h}, \\
5 / 1.1 \mathrm{E}+6 \mathrm{~h} \\
{[12]}\end{array}$ & & & & & $2.1 \mathrm{E}-5 / \mathrm{h}(3.0)$ \\
\hline Fails to run & & $\begin{array}{l}16.6 / 1.1 \mathrm{E}+6 \mathrm{~h}, \\
2.1 / 1.1 \mathrm{E}+6 \mathrm{~h} \\
{[12]}\end{array}$ & & & & & $8.5 \mathrm{E}-6 / \mathrm{h}(2.8)$ \\
\hline $\begin{array}{l}\text { Synchro } \\
\text { Failure }\end{array}$ & & $4.9 / 3.8 \mathrm{E}+5 \mathrm{~h}$ & & & & & $1.4 \mathrm{E}-5 / \mathrm{h}(10)$ \\
\hline $\begin{array}{l}\text { Transformer } \\
\text { Power } \\
\text { Failure } \\
\end{array}$ & & $\begin{array}{l}3.0 / 7.8 \mathrm{E}+6 \mathrm{~h}, \\
077.6 \mathrm{E}+5 \mathrm{~h} \\
{[14]}\end{array}$ & $\begin{array}{l}0 / 6.9 \mathrm{E}+5 \mathrm{~h}, \\
0 / 8.4 \mathrm{E}+5 \mathrm{~h} \\
{[7]}\end{array}$ & & & & $3.5 \mathrm{E}-7 / \mathrm{h}(10)$ \\
\hline $\begin{array}{l}\text { Instrumentation/Control } \\
\text { Failure }\end{array}$ & & & & & & & \\
\hline
\end{tabular}

Table 4e. Category 2 data for electrical system. 


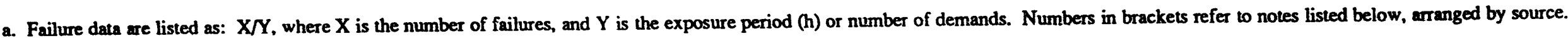
b. Aggregated results are mean and error factor (in parentheses). The error factor is the 95th percentile/50th percentile.

Notes

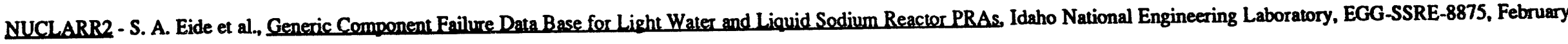
1990.

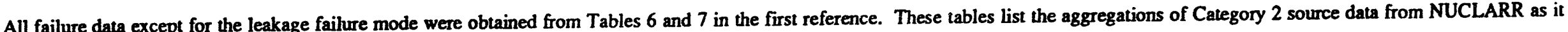
existed in August 1989.

1. Diesel generator fail to start data used.

2. Diesel generator fail to nun data used.

3. Battery data used.

4. Battery charger data used.

5. Power circuit breaker fail to operate and fail to stan data used.

6. Power electronics (inverter) data used.

NPRD-3 - M. J. Rossi, Nonelectronic Parts Reliability Data, Reliability Analysis Center, Rome Air Development Center, NPRD-3, 1985.

failure data

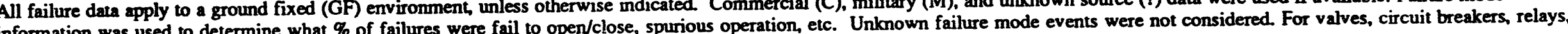
and switches, the following mapping of NPRD-3 failure modes into the failure modes of interest was used:

Fail to open/close/oper: - binding, breach (not for valves), contaminated, cracked/fractured, no movement, no operation, seized, stuck open, and stuck closed

Spurious operation - arcing, collapsed, false response, improper flow, intermittent, opened, overheated, and shorted

Plugs - none identified

Leakage (internal) - none identified except for leaking (check valve only)

Leakage (extemal) - breach (valves only), leaking

Degraded/incipient (not catastrophic) - degraded, drift, fatigue, improper timing, noisy, other, out of adjustment, out of specification, unstable.

For pumps, fans, and diesel generators, the following mapping was used:

Fail to start - contaminated, cracked/fractured, no movement, no operation

Fail to run - binding, broken, displaced, false response, improper flow, intermittent, overheated, seized, shorted, stuck closed, stuck open 
WSC-TR-93-262

Overspeed (subset of fail to run) - improper flow (one-half of events used), stuck open

Fail to stop - none identified

Leakage (external) - breach (not for diesel generator), leaking

Degraded/incipient (not catastrophic) - corroded, drift, noisy, out of adjustment, out of specification, worn out.

1. Diesel generator data used (p. 76). Failure mode information (p. 328) indicates that $100 \%$ of failures are fails to start.

2. De generator data used (p. 75). Failure mode information (p. 326) indicates that $44.4 \%$ of failures are fails to start and $22.2 \%$ are fails to run.

3. Turbine generator data used (p. 77). Failure mode information (p. 330) indicates that $10.5 \%$ of failures are fails to start and $15.8 \%$ are fails to run.

4. Rechargeable battery data used (p. 33). Failure mode information (p. 279) indicates that $36.4 \%$ of failures are catastrophic.

5. General circuit breaker data used (p. 43). Failure mode information (p. 283) indicates that $65.7 \%$ of failures are fails to open/close and $15.4 \%$ are spurious operation.

6. General relay data used (p. 117). Faihure mode information (p. 311) indicates that $16.8 \%$ of failures are fails to open/close and $63.5 \%$ are spurious operation.

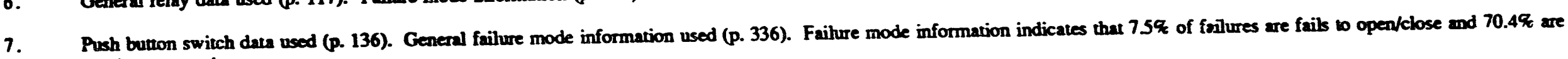
spurious operation.

8. Rotary switch data used (p. 137). See Nore 7 for faihure mode information.

9. Key switch data used (p. 135). See Note 7 for failure mode information.

10. Fuse data used (p. 69). Failure mode information (p. 285) indicates that $75 \%$ of failures are fails wo open and $10 \%$ are premature opening.

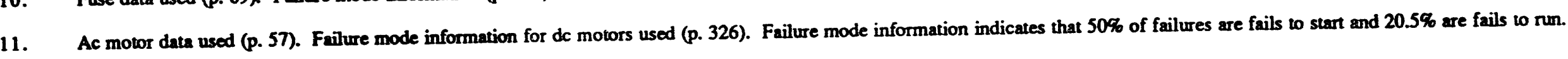

12. De motor data used (p. 58). See Note 11 for failure mode information.

13. General synchro data used (p. 141). Failure mode information (p. 331) indicates that $81.7 \%$ of failures are catastrophic.

14. General transformer data used (p. 148). Failure mode information (p. 319) indicates that $60 \%$ of failures are catastrophic.

OREDA - Offshore Reliability Data Handbook, Offshore Reliability Data (OREDA), Norway, 1984.

All failure information was taken from the critical failure mode category, unless otherwise indicated.

1. Diesel engine driven generator set data used (pp. 247 and 249). Failed while running and improper operation data used for fails to run. Operation time used for fails to run.

2. 
3. Battery data used (p. 263). Inadequate output and no output during emergency mode of operation data used for failure.

4. Rectifier (p. 259) and battery charger (p. 261) data used.

5. Circuit breaker data used (pp. 265, 267, and 269).

6. Inverter data used (p. 257). No output and failed to transfer data used for failure.

7. Transformer data used (pp. 253 and 255).

WIN-330 - J. N. Wilkinson et al., Idaho Chemical Processing Plant Failure Rate Database, Idaho National Engineering Laboratory, WIN-330, October 1991.

All failure information was taken from Appendix C, p. 32.

TRITIUM - L. C. Cadwallader and M. A. Stolpe Gavett, Tritium Waste Treatment System Component Failure Data Analysis from June 18. 1984 to December 31. 1989, Idaho National

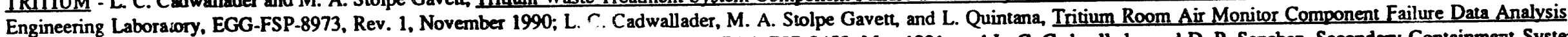

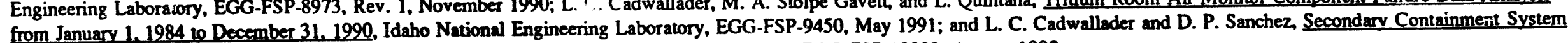
Component Failure Data Analysis from 1984 te 1991. Idaho National Engineering Laboratory, EGG-FSP-10323, August 1992.

LNG1 - D. W. Johnson ad J. R. Welker, Development of an Improved LNG Plant Failure Rate Data Base, Gas Research Institute, PB82-153503, September 1981.

\section{Aggregated Results}

1. Conbined result calculated for per hour data. OREDA data used to partition among failure modes. 
WSRC-TR-93-262

\begin{tabular}{|c|c|c|c|c|c|c|c|}
\hline \multirow{2}{*}{ System/Component/Failure Mode } & \multicolumn{7}{|c|}{ Failure Data (Category 2 Sources) ${ }^{\mathrm{a}}$} \\
\hline & NUCLARR2 & NPRD-3 & OREDA & WIN-330 & TRITIUM & LNG1 & $\begin{array}{c}\text { Aggregated } \\
\text { Results }^{b}\end{array}$ \\
\hline $\begin{array}{c}\text { Instrumentation and Control } \\
\text { Alarm/Annunciator } \\
\text { Fails to alarm }\end{array}$ & & & $\begin{array}{l}11 / 3.2 \mathrm{E}+5 \mathrm{~h} \\
{[1]}\end{array}$ & & & & $3.6 \mathrm{E}-5 / \mathrm{h}(10)$ \\
\hline Spurious operation & & & $\begin{array}{l}1 / 3.2 \mathrm{E}+5 \mathrm{~h} \\
{[1]}\end{array}$ & & & & $4.7 \mathrm{E}-6 / \mathrm{h}(10)$ \\
\hline $\begin{array}{l}\text { Sensor/Transmitter/ } \\
\text { Transducer/Process Switch } \\
\text { Temperature } \\
\text { Failure } \\
\end{array}$ & & $\begin{array}{l}1.2 / 5.1 \mathrm{E}+6 \mathrm{~h} \\
{[1]}\end{array}$ & $\begin{array}{l}0 / 1.9 \mathrm{E}+6 \mathrm{~h}, \\
8 / 1.2 \mathrm{E}+6 \mathrm{~h} \\
{[2]}\end{array}$ & $\begin{array}{l}7 / 1.5 \mathrm{E}+6 \mathrm{~h} \\
{[1]}\end{array}$ & $\begin{array}{l}0 / 5.2 \mathrm{E}+5 \mathrm{~h} \\
{[1]}\end{array}$ & $\begin{array}{l}2 / 2.6 \mathrm{E}+6 \mathrm{~h} \\
0 / 8.4 \mathrm{E}+6 \mathrm{~h} \\
{[1]}\end{array}$ & $8.6 \mathrm{E}-7 / \mathrm{h}(7.9)$ \\
\hline $\begin{array}{l}\text { Pressure } \\
\text { Faihure }\end{array}$ & & $\begin{array}{l}26.2 / 4.6 \mathrm{E}+7 \mathrm{~h} \\
{[2]}\end{array}$ & $\begin{array}{l}4 / 7.2 \mathrm{E}+6 \mathrm{~h}, \\
8 / 4.3 \mathrm{E}+6 \mathrm{~h}, \\
14 / 5.0 \mathrm{E}+6 \mathrm{~h}, \\
0 / 3.5 \mathrm{E}+6 \mathrm{~h}, \\
2 / 3.5 \mathrm{E}+6 \mathrm{~h}, \\
3 / 5.5 \mathrm{E}+6 \mathrm{~h} \\
{[3]}\end{array}$ & $\begin{array}{l}3 / 1.0 \mathrm{E}+6 \mathrm{~h} \\
{[2]}\end{array}$ & $\begin{array}{l}3 / 5.7 \mathrm{E}+5 \mathrm{~h} \\
{[2]}\end{array}$ & & $8.3 \mathrm{E}-7 / \mathrm{h}(3.1)$ \\
\hline $\begin{array}{l}\text { Differential Pressure } \\
\text { Faihure }\end{array}$ & & & 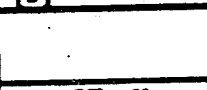 & $\begin{array}{l}2 / 9.2 \mathrm{E}+5 \mathrm{~h} \\
{[3]}\end{array}$ & & & $2.7 \mathrm{E}-6 / \mathrm{h}(10)$ \\
\hline $\begin{array}{l}\text { Fow } \\
\text { Faikure }\end{array}$ & & $\begin{array}{l}38.5 / 1.5 \mathrm{E}+7 \mathrm{~h} \\
{[3]}\end{array}$ & $\begin{array}{l}1 / 1.5 \mathrm{E}+6 \mathrm{~h} . \\
0 / 1.1 \mathrm{E}+5 \mathrm{~h}, \\
1 / 2.4 \mathrm{E}+5 \mathrm{~h}, \\
0 / 6.6 \mathrm{E}+5 \mathrm{~h}, \\
4 / 2.7 \mathrm{E}+6 \mathrm{~h} . \\
0 / 4.2 \mathrm{E}+5 \mathrm{~h}, \\
21 / 3.4 \mathrm{E}+6 \mathrm{~h}, \\
0 / 3.4 \mathrm{E}+5 \mathrm{~h} \\
{[4]}\end{array}$ & $\begin{array}{l}3 / 7.6 \mathrm{E}+5 \mathrm{~h} \\
{[4]}\end{array}$ & & & $2.9 \mathrm{E}-6 / \mathrm{h}(2.0)$ \\
\hline $\begin{array}{l}\text { Level } \\
\text { Failure }\end{array}$ & & $\begin{array}{l}2.3 / 7.6 \mathrm{E}+5 \mathrm{~h} \\
{[4]}\end{array}$ & $\begin{array}{l}9 / 2.6 \mathrm{E}+7 \mathrm{~h}, \\
4 / 1.2 \mathrm{E}+7 \mathrm{~h} \\
1 / 4.6 \mathrm{E}+6 \mathrm{~h} \\
{[5]}\end{array}$ & $\begin{array}{l}9 / 4.1 \mathrm{E}+6 \mathrm{~h} \\
{[5]}\end{array}$ & $\begin{array}{l}0 / 9.5 \mathrm{E}+4 \mathrm{~h} \\
{[3]}\end{array}$ & & $5.3 \mathrm{E}-7 \mathrm{~h}(3.7)$ \\
\hline $\begin{array}{r}\text { Humidity } \\
\text { Failure }\end{array}$ & & $\begin{array}{l}2.3 / 2.4 \mathrm{E}+5 \mathrm{~h} \\
{[5]}\end{array}$ & & & & & $1.2 \mathrm{E}-5 / \mathrm{h}(10)$ \\
\hline Failure & & & & & & & \\
\hline $\begin{array}{l}\text { Oxygen Concentration } \\
\text { Failure }\end{array}$ & & & & & $\begin{array}{l}3 / 3.7 \mathrm{E}+5 \mathrm{~h} \\
{[4]}\end{array}$ & & $9.5 \mathrm{E}-6 / \mathrm{h}(10)$ \\
\hline $\begin{array}{l}\mathrm{CO}_{2} \text { Concentration } \\
\text { Failure }\end{array}$ & & & & & & & \\
\hline $\begin{array}{l}\text { Hydrogen Concentration } \\
\text { Failure }\end{array}$ & & & & & & & \\
\hline
\end{tabular}


WSRC-TR-93-262

\begin{tabular}{|c|c|c|c|c|c|c|c|}
\hline \multirow[t]{2}{*}{ System/Component/Failure Mode } & \multicolumn{7}{|c|}{ Failure Data (Category 2 Sources) $)^{a}$} \\
\hline & NUCLARR2 & NPRD-3 & OREDA & WIN-330 & TRITIUM & LNG1 & $\begin{array}{l}\text { Aggregated } \\
\text { Results } b\end{array}$ \\
\hline \multicolumn{8}{|l|}{$\begin{array}{l}\text { Nitrogen Concentration } \\
\text { Failure }\end{array}$} \\
\hline $\begin{array}{l}\text { Hydrocarbon Concen- } \\
\text { tration } \\
\text { Failure } \\
\end{array}$ & & & $\begin{array}{l}318 / 2.9 \mathrm{E}+7 \mathrm{~h} \\
{[6]}\end{array}$ & & & $\begin{array}{l}44 / 1.7 \mathrm{E}+7 \mathrm{~h} \\
{[2]}\end{array}$ & $7.9 \mathrm{E}-6 / \mathrm{h}(2.2)$ \\
\hline \multicolumn{8}{|l|}{$\begin{array}{l}\text { Helium Concentration } \\
\text { Failure } \\
\end{array}$} \\
\hline \multicolumn{8}{|l|}{$\begin{array}{l}\text { Speed } \\
\text { Failure }\end{array}$} \\
\hline \multicolumn{8}{|l|}{$\begin{array}{l}\text { Seismic } \\
\text { Failure }\end{array}$} \\
\hline $\begin{array}{l}\text { Radiation } \\
\text { Failure }\end{array}$ & & & & $\begin{array}{l}11 / 2.1 \mathrm{E}+6 \mathrm{~h}, \\
18 / 1.4 \mathrm{E}+6 \mathrm{~h} \\
{[6]}\end{array}$ & $\begin{array}{l}17 / 1.9 \mathrm{E}+5 \mathrm{~h}, \\
2 / 4.6 \mathrm{E}+5 \mathrm{~h} \\
{[5]}\end{array}$ & & $1.2 \mathrm{E}-5 / \mathrm{h}(5.7)$ \\
\hline $\begin{array}{c}\text { Indicator } \\
\text { Faihure } \\
\end{array}$ & & $\begin{array}{l}2.6 / 2.1 \mathrm{E}+5 \mathrm{~h} \\
{[6]}\end{array}$ & & & & & $1.5 \mathrm{E}-5 / \mathrm{h}(10)$ \\
\hline \multicolumn{8}{|l|}{$\begin{array}{l}\text { Amplifier } \\
\text { Failure }\end{array}$} \\
\hline \multicolumn{8}{|l|}{$\begin{array}{l}\text { Modifier/Signal Conditioner } \\
\text { Failure }\end{array}$} \\
\hline \multicolumn{8}{|l|}{$\begin{array}{l}\text { Logic Module } \\
\text { Failure }\end{array}$} \\
\hline \multicolumn{8}{|l|}{$\begin{array}{c}\text { Recorder } \\
\text { Failure }\end{array}$} \\
\hline $\begin{array}{l}\text { Sampler } \\
\text { Failure }\end{array}$ & & & & $\begin{array}{l}27 / 2.5 \mathrm{E}+6 \mathrm{~h}, \\
15 / 1.2 \mathrm{E}+6 \mathrm{~h} \\
{[7]}\end{array}$ & & & $1.2 \mathrm{E}-5 \mathrm{~h}(10)$ \\
\hline $\begin{array}{c}\text { Analyzer } \\
\text { Failure } \\
\end{array}$ & & & & $\begin{array}{l}1 / 2.5 \mathrm{E}+5 \mathrm{~h} \\
{[8]}\end{array}$ & & & $6.0 \mathrm{E}-6 / \mathrm{h}(10)$ \\
\hline \multicolumn{8}{|l|}{$\begin{array}{l}\text { Timer } \\
\text { Failure } \\
\end{array}$} \\
\hline $\begin{array}{l}\text { Gas Chromatograph } \\
\text { Faihure }\end{array}$ & & & & & $\begin{array}{l}3 / 4.8 \mathrm{E}+4 \mathrm{~h} \\
{[6]}\end{array}$ & & $7.3 \mathrm{E}-5 / \mathrm{h}(10)$ \\
\hline $\begin{array}{l}\text { Voltage Regulator } \\
\text { Failure }\end{array}$ & & $\begin{array}{l}10 / 3.3 \mathrm{E}+6 \mathrm{~h} \\
{[7]}\end{array}$ & & & & & $3.2 \mathrm{E}-6 / \mathrm{h}(10)$ \\
\hline $\begin{array}{c}\text { Transmitter } \\
\text { Failure }\end{array}$ & & & & & & & \\
\hline
\end{tabular}




\begin{tabular}{|c|c|c|c|c|c|c|c|}
\hline \multirow{2}{*}{ System/Component/Failure Mode } & \multicolumn{7}{|c|}{ Failure Data (Category 2 Sources) } \\
\hline & NUCLARR2 & NPRD-3 & OREDA & WIN-330 & TRITUM & LNG1 & $\begin{array}{c}\text { Aggregated } \\
\text { Results }\end{array}$ \\
\hline $\begin{array}{l}\text { Transducer } \\
\text { Failure } \\
\end{array}$ & & $\begin{array}{l}0.7 / 1.3 \mathrm{E}+6 \mathrm{~h} \\
{[8]}\end{array}$ & & & & & $9.2 \mathrm{E}-7 / \mathrm{h}(10)$ \\
\hline $\begin{array}{l}\text { Programmable Logic Controller } \\
\text { Failure }\end{array}$ & & & $\begin{array}{l}8.2 / 2.7 \mathrm{E}+5 \mathrm{~h} \\
{[7]}\end{array}$ & & & & $3.2 \mathrm{E}-5 / \mathrm{h}(10)$ \\
\hline
\end{tabular}

Table 4f. Category 2 data for instrumentation and control system.

a. Failure data are listed as: $X / Y$, where $X$ is the number of failures, and $Y$ is the exposure period $(h)$ or number of demands. Numbers in brackets refer to notes listed below, arranged by source.

b. Aggregated results are mean and error factor (in parentheses). The error factor is the 95 th percentile/50th percentile.

Notes

NUCLARR2 . S. A. Eide a al, Generic Component Failure Dara Base for Light Water and Liquid Sodium Reactor PRAs Idaho National Engineering Laboratory, EGG-SSRE-8875, Fetruary 1990.

All failure data except for the leakage failure mode were obtained from Tables 6 and 7 in the first reference. These tables list the aggregations of Category 2 source data from NUCLARR as it existed in August 1989.

NPRD-3 - M. J. Rossi, Nonelectronic Parts Reliability Data Reliability Analysis Center, Rome Air Development Center, NPRD-3, 1985.

All failure data apply to a All failure data apply to a ground fixed (GF) environment, unless otherwise indicated. Commercial (C), Unknown failure mode events were not considered. For valves, circuit breakers, relays, information was used to determine what \% of failures were fail to opene failure modes of interest was used:

Fail to open/close/operate - binding, breach (not for valves), contaminated, cracked/fractured, no movement, no operation, seized, stuck open, and stuck closed

Spurious operation - arcing, collapsed, false response, improper flow, intermittent, opened, overheated, and shorted

Plugs - none identified

Leakage (internal) - none identified except for leaking (check valve only)

Leakage (extemal) - breach (valves only), leaking

Degraded/incipient (not catastrophic) - degraded, drift, fatigue, improper timing, noisy, other, out of adjustment, out of specification, unstable. 
For pumps, fans, and diesel generators, the following mapping was used:

Fail to start - contaminated, cracked/fractured, no movement, no operation

Fail to run - binding, broken, displaced, false response, improper flow, intermittent, overheated, seized, shorted, stuck closed, stuck open

Overspeed (subset of fail to run) - improper flow (one-half of events used), stuck open

Fail to stop - none identified

Leakage (external) - breach (not for diesel generator), leaking

Table 4f. Category 2 data for instrumentation and control system.

Degraded/incipient (not catastrophic) - corroded, drift, noisy, out of adjustment, out of specification, wom out.

1. Temperature switch data used (p. 139). General switch failure mode information used (p. 336). Failure mode information indicates 58.3\% of failures are catastrophic.

2. Pressure switch data used (p. 136). See Note 1 for failure mode information.

3. Flow switch data used (p. 133). See Note 1 for failure mode information.

4. Level switch data used (p. 135). See Note 1 for faihure mode information.

5. Humidity switch data used (p. 134). See Note 1 for failure mode information.

6. Elapsed time indicator data used (p. 86). CRT display failure mode information used (p. 307). Faihure mode information indicates 28.6\% of failures are catastrophic.

7. Voltage regulator data used (p. 114). No failure mode information available.

8. General transducer data used (p. 146). Faihure mode information (p. 338) indicates $13.2 \%$ of failures are catastrophic.

QREDA - Offshore Reliability Data Handbook, Offshore Reliability Data (OREDA), Norway, 1984.

All failure information was taken from the critical failure mode category, unless otherwise indicated.

1. Alarm system data used (p. 133).

2. Sensor/pneumatic switch (p. 61) sensor/electric switch (p. 63), and sensor/transducer (p. 65) data used.

3. Sensor/pneumatic switch (pp. 49 and 51), sensor/electric switch (p. 53), and sensor/transducer (pp. 55, 57, and 59) data used.

4. Sensor/pneumatic switch (pp. 67 and 69), sensor/electric switch (pp. 71 and 73), and sensor/transducer (pp. 75, 77, 79, and 81) data used.

5. Sensor/pneumatic switch (p. 83), sensor/electric switch (p. 85), and sensor/transducer (p. 87) data used. 
Hydrocarbon gas detector data used.

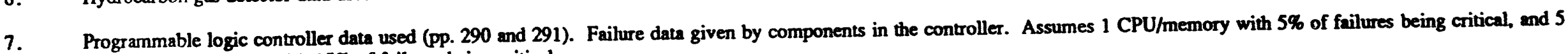
input and output cards with $15 \%$ of failures being critical.

WIN-330 - J. N. Wilkinson et al., Idaho Chemical Presessine Plant Failure Rate Database, Idaho National Engineering Laboratory, WIN-330, October 1991.

All failure information was taken from Appendix C, p. 32.

Table 4f. Categrry 2 data for instrumentation and control system.

1. Temperature (loop) data used. Fail failure mode data used.

2. Pressure nnm) data used. Fail failure mode data used.

3. Differential pressure (loop) data used. Fail failure mode data used.

4. Flow (gas loop) dma used. Fail failure mode data used.

5. Level (:op) data used. Fail failure mode data used

6. Radiation (general loop) and radiation (criticality, bop) data used Fail faihure mode dana used

7. Samplet system (non-acid and acid fluid) data used.

8. Analysis (loop) data used. Fail faihure mode data used

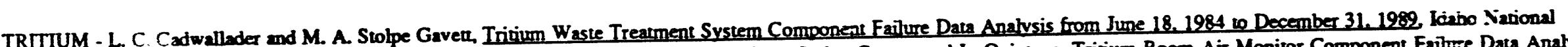

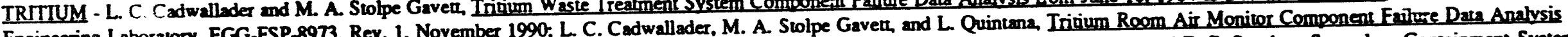

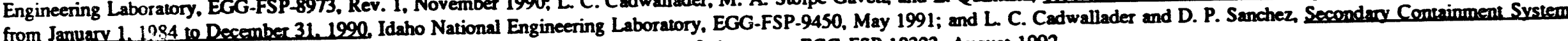
from January 1.1984 to December 31. 1920 to 1991 . Idaho National Engineering Laboratory. EGG-FSP-10323, August 1992.

1. Temperature monitor data used (Table A-1, first reference).

2. Pressure monitor data used (Table A-1, first reference).

3. Level monitor data used (Table A-1, first reference).

4. Oxygen monitor date used (Table A-1, first reference).

5. Radiatio: monitor data used (Table A-1, first reference and p. A-3, second reference).

6. Gas chromatograph data used (Table A-1, first reference). 
LNG1 - D. W. Johnson and J. R. Welker, Development of an Improved LNG Plant Failure Rate Data Base, Gas Research Institute, PB82-153503, September 1981.

1. First set of data is for low temperature detector (p. 9). Second set of data is for high temperature detector (p. 9).

2. Gas detector data used (p. 9). 
WSRC-TR-93-262

\begin{tabular}{|c|c|c|c|c|c|c|c|}
\hline \multirow[t]{2}{*}{ System/Component/Failure Mode } & \multicolumn{7}{|c|}{ Faihure Dai= (Calegory 3 Sources) ${ }^{\mathrm{a}}$} \\
\hline & WASH-1400 & CCPS & IEEE & LNG2 & EPRII & EPR12 & $\begin{array}{l}\text { Aggregated } \\
\text { Results b }\end{array}$ \\
\hline $\begin{array}{l}\text { Water } \\
\text { Valve (Standby or Safety) } \\
\text { Manual } \\
\text { Fails to open/close }\end{array}$ & & & $\begin{array}{l}6.0 \mathrm{E}-5 / \mathrm{d} \\
{[1]}\end{array}$ & & & & $6.0 \mathrm{E}-5 / \mathrm{d}$ \\
\hline Plugs & $3.8 \mathrm{E}-7 / \mathrm{h}(3)$ & & & & & & $3.8 \mathrm{E}-7 / \mathrm{h}(3)$ \\
\hline \multicolumn{8}{|l|}{ Leakage (internal) } \\
\hline \multicolumn{8}{|l|}{ Rupture ( .mal) } \\
\hline Leakage : atemal) & & & $\begin{array}{l}2.0 \mathrm{E}-8 / \mathrm{h} \\
\text { [1] }\end{array}$ & & & & $2.0 \mathrm{E}-8 / \mathrm{h}$ \\
\hline Rupture vernal) & $2.7 \mathrm{E}-8 / \mathrm{h}(10)$ & & & & & & $2.7 \mathrm{E}-8 / \mathrm{h}(10)$ \\
\hline $\begin{array}{r}\text { Check } \\
\text { I.:3 to open }\end{array}$ & $1.3 \mathrm{E}-4 / \mathrm{d}(3)$ & & $\begin{array}{l}\text { 6.0E-5/d } \\
\text { [i] }\end{array}$ & & & & $8.8 \mathrm{E}-5 / \mathrm{d}(3.0)$ \\
\hline \multicolumn{8}{|l|}{ Fails to close } \\
\hline \multicolumn{8}{|l|}{ Plugs } \\
\hline Leakage (internal) & & & $\begin{array}{l}5.0 \mathrm{E}-7 / \mathrm{h} \\
\text { [1] }\end{array}$ & & & & $5.0 \mathrm{E}-7 / \mathrm{h}(10)$ \\
\hline Rupture (internal) & $3.8 \mathrm{E}-7 \mathrm{~h}(3)$ & & $\begin{array}{l}5.0 \mathrm{E}-8 / \mathrm{h} \\
\text { [1] }\end{array}$ & & & & $1.9 \mathrm{E}-7 / \mathrm{h}(5.4)$ \\
\hline \multicolumn{8}{|l|}{ Líakage (external) } \\
\hline Rupture (extemal) & $2.7 \mathrm{E}-8 / \mathrm{h}(10)$ & & & & & & $2.7 \mathrm{E}-8 / \mathrm{h}(10)$ \\
\hline $\begin{array}{l}\text { Motor-Operated } \\
\text { Fails to open/close }\end{array}$ & $1.3 \mathrm{E}-3 / \mathrm{d}(3)$ & & $\begin{array}{l}6.0 \mathrm{E}-3 / \mathrm{d} \\
{[1]}\end{array}$ & & & & $3.0 \mathrm{E}-3 / \mathrm{d}(3.5)$ \\
\hline \multicolumn{8}{|l|}{ Spurious operation } \\
\hline Plugs & $3.8 \mathrm{E}-7 / \mathrm{h}(3)$ & & $\begin{array}{l}3.0 \mathrm{E}-8 / \mathrm{h} \\
\text { [1] }\end{array}$ & & & & $1.9 \mathrm{E}-7 \mathrm{~h}(8.1)$ \\
\hline \multicolumn{8}{|l|}{ Leakage (internal) } \\
\hline \multicolumn{8}{|l|}{ Rupare (internal) } \\
\hline Leakage (external) & & & $\begin{array}{l}1.0 \mathrm{E}-7 \mathrm{~h} \\
{[1]}\end{array}$ & & & & $1.0 \mathrm{E}-7 / \mathrm{h}(10)$ \\
\hline Rupture (external) & $2.7 \mathrm{E}-8 / \mathrm{h}(10)$ & & & & & & $2.7 \mathrm{E}-8 / \mathrm{h}(10)$ \\
\hline
\end{tabular}


WSRC-TR-93-262

\begin{tabular}{|c|c|c|c|c|c|c|c|}
\hline \multirow[t]{2}{*}{ System/Component/Failure Mode } & \multicolumn{7}{|c|}{ Failure Data (Category 3 Sources) } \\
\hline & WASH-1400 & $\overline{C C P S}$ & IEEE & LNG2 & EPRI1 & EPR12 & $\begin{array}{l}\text { Aggregated } \\
\text { Results }\end{array}$ \\
\hline $\begin{array}{l}\text { Air-Operated } \\
\text { Fails to open/close }\end{array}$ & $3.8 \mathrm{E}-4 / \mathrm{d}(3)$ & & $\begin{array}{l}2.0 \mathrm{E}-3 / \mathrm{d} \\
{[1]}\end{array}$ & & & & $9.8 \mathrm{E}-4 / \mathrm{d}(3.9)$ \\
\hline \multicolumn{8}{|l|}{ Spurious operation } \\
\hline Plugs & $3.8 \mathrm{E}-7 / \mathrm{h}(3)$ & & & & & & $3.8 \mathrm{E}-7 / \mathrm{h}(3)$ \\
\hline \multicolumn{8}{|l|}{ Leakage (internal) } \\
\hline \multicolumn{8}{|l|}{ Rupturc (internal) } \\
\hline Leakage (external) & & & $\begin{array}{l}1.0 \mathrm{E}-7 / \mathrm{h} \\
{[1]}\end{array}$ & & & & $1.0 \mathrm{E}-7 \mathrm{~h}(10)$ \\
\hline Rupture (external) & $2.7 \mathrm{E}-8 / \mathrm{h}(10)$ & & & & & & $2.7 E-8 / h(10)$ \\
\hline $\begin{array}{l}\text { Solenoid-Oprrated } \\
\text { Fails to open/close }\end{array}$ & $1.3 \mathrm{E}-3 / \mathrm{d}(3)$ & & & & & & $1.3 \mathrm{E}-3 / \mathrm{d}(3)$ \\
\hline \multicolumn{8}{|l|}{ Spurious operation } \\
\hline Plugs & $\begin{array}{l}3.8 \mathrm{E}-7 / \mathrm{h}(3) \\
{[1]}\end{array}$ & & & & & & $3.8 \mathrm{E}-7 / \mathrm{h}(3)$ \\
\hline \multicolumn{8}{|l|}{ Leakage (internal) } \\
\hline \multicolumn{8}{|l|}{ Rupkure (intemal) } \\
\hline \multicolumn{8}{|l|}{ Leakagc (citernal) } \\
\hline Rupture (external) & $2.7 \mathrm{E}-8 / \mathrm{h}(10)$ & & & & & & $2.7 \mathrm{E}-8 / \mathrm{h}(10)$ \\
\hline $\begin{array}{l}\text { Safety/Relicf } \\
\text { Fails to open }\end{array}$ & $1.3 \mathrm{E}-5 / \mathrm{d}(3)$ & & $\begin{array}{l}4.0 E-3 / d \\
{[1.2]}\end{array}$ & & & & $1.5 \mathrm{E}-3 / \mathrm{d}(30)$ \\
\hline \multicolumn{8}{|l|}{ Fails to reclose } \\
\hline Leakage (iniemal) & $1.3 \mathrm{E}-5 / \mathrm{h}(3)$ & & & & & & $1.3 \mathrm{E}-5 / \mathrm{h}(3)$ \\
\hline Rupture (intemal) & & & $\begin{array}{l}3.0 \mathrm{E}-6 / \mathrm{h} \\
{[1,2]}\end{array}$ & & & & $3.0 \mathrm{E}-6 / \mathrm{h}(10)$ \\
\hline \multicolumn{8}{|l|}{ Leakage (external) } \\
\hline \multicolumn{8}{|l|}{ Rupture (external) } \\
\hline $\begin{array}{l}\text { Vacunum-Breaker } \\
\text { Fails to open }\end{array}$ & $3.8 \mathrm{E}-5 / \mathrm{d}(3)$ & & & & & & $3.8 E-5 / d(3)$ \\
\hline
\end{tabular}


WSRC-TR-93-262

Table 5a. Category 3 estimates for water system (continued).

\begin{tabular}{|c|c|c|c|c|c|c|c|}
\hline \multirow[t]{2}{*}{ System/Component/Faihure Mode } & \multicolumn{7}{|c|}{ Faihure Data (Category 3 Sources) } \\
\hline & WASH-1400 & CCPS & IEEE & LNG2 & EPRI1 & EPR12 & $\begin{array}{l}\text { Aggregated } \\
\text { Results }\end{array}$ \\
\hline \multicolumn{8}{|l|}{ Fails to reclose } \\
\hline \multicolumn{8}{|l|}{ sage (internal) } \\
\hline \multicolumn{8}{|l|}{ Rupture (intemal) } \\
\hline \multicolumn{8}{|l|}{ Leakage (external) } \\
\hline \multicolumn{8}{|l|}{$\begin{array}{l}\text { Valve (Control) } \\
\text { Motor-Operated } \\
\text { Fails open }\end{array}$} \\
\hline \multicolumn{8}{|l|}{ Fails closed } \\
\hline \multicolumn{8}{|l|}{ Fails to respond } \\
\hline \multicolumn{8}{|l|}{ Plugs } \\
\hline \multicolumn{8}{|l|}{ Leakage (external) } \\
\hline \multicolumn{8}{|l|}{ Rupture (external) } \\
\hline \multicolumn{8}{|l|}{$\begin{array}{l}\text { Air-Operated } \\
\text { Fails open }\end{array}$} \\
\hline \multicolumn{8}{|l|}{ Fails closed } \\
\hline \multicolumn{8}{|l|}{ Fails to respond } \\
\hline \multicolumn{8}{|l|}{ Phigs } \\
\hline \multicolumn{8}{|l|}{ Leakage (extermal) } \\
\hline \multicolumn{8}{|l|}{ Rupture (external) } \\
\hline \multicolumn{8}{|l|}{$\begin{array}{l}\text { Solenoid-Operated } \\
\text { Fails open }\end{array}$} \\
\hline \multicolumn{8}{|l|}{ Fails closed } \\
\hline \multicolumn{8}{|l|}{ Fn: to respond } \\
\hline Phin & & & & & & & \\
\hline
\end{tabular}



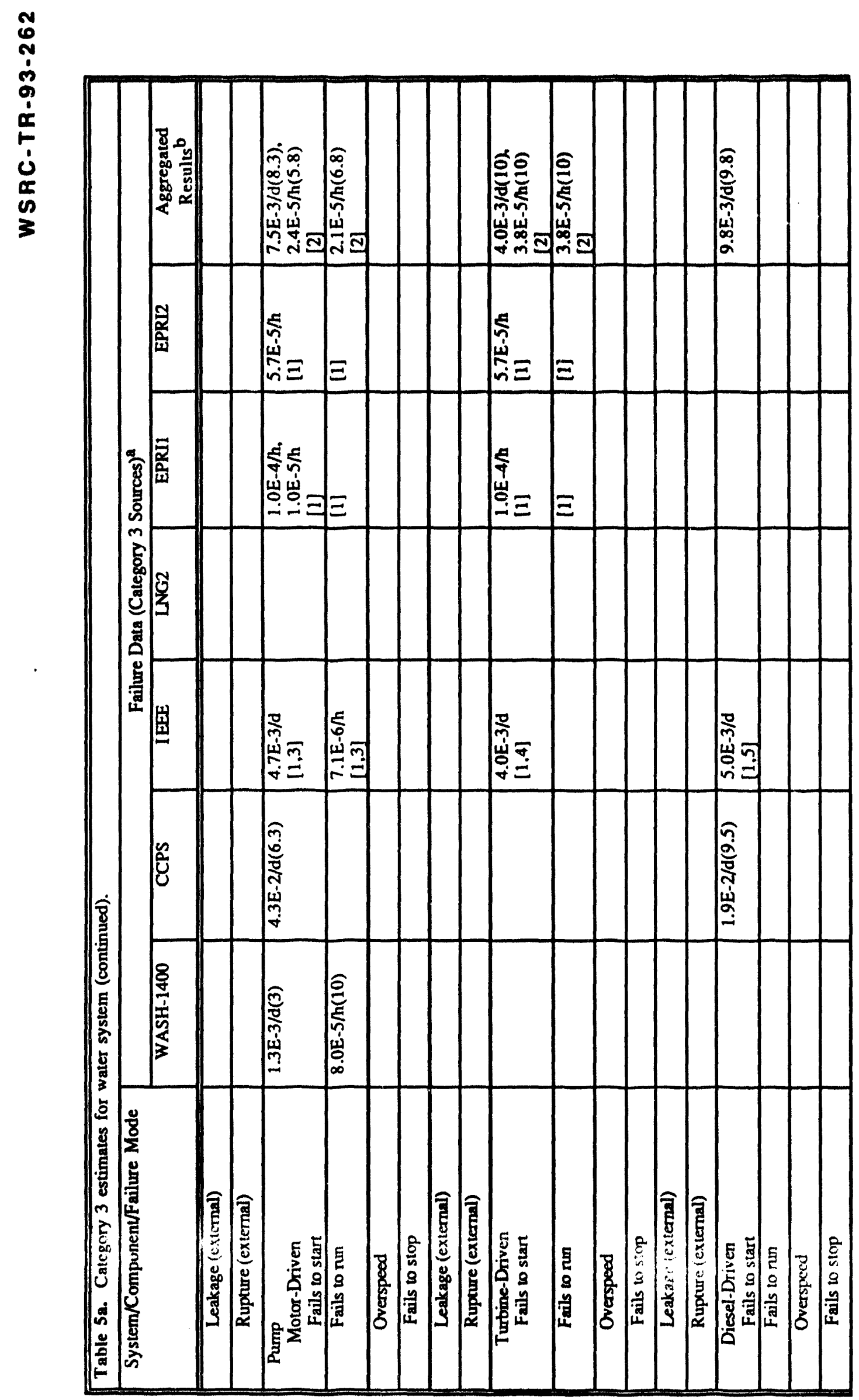

$\stackrel{N}{N}$ 


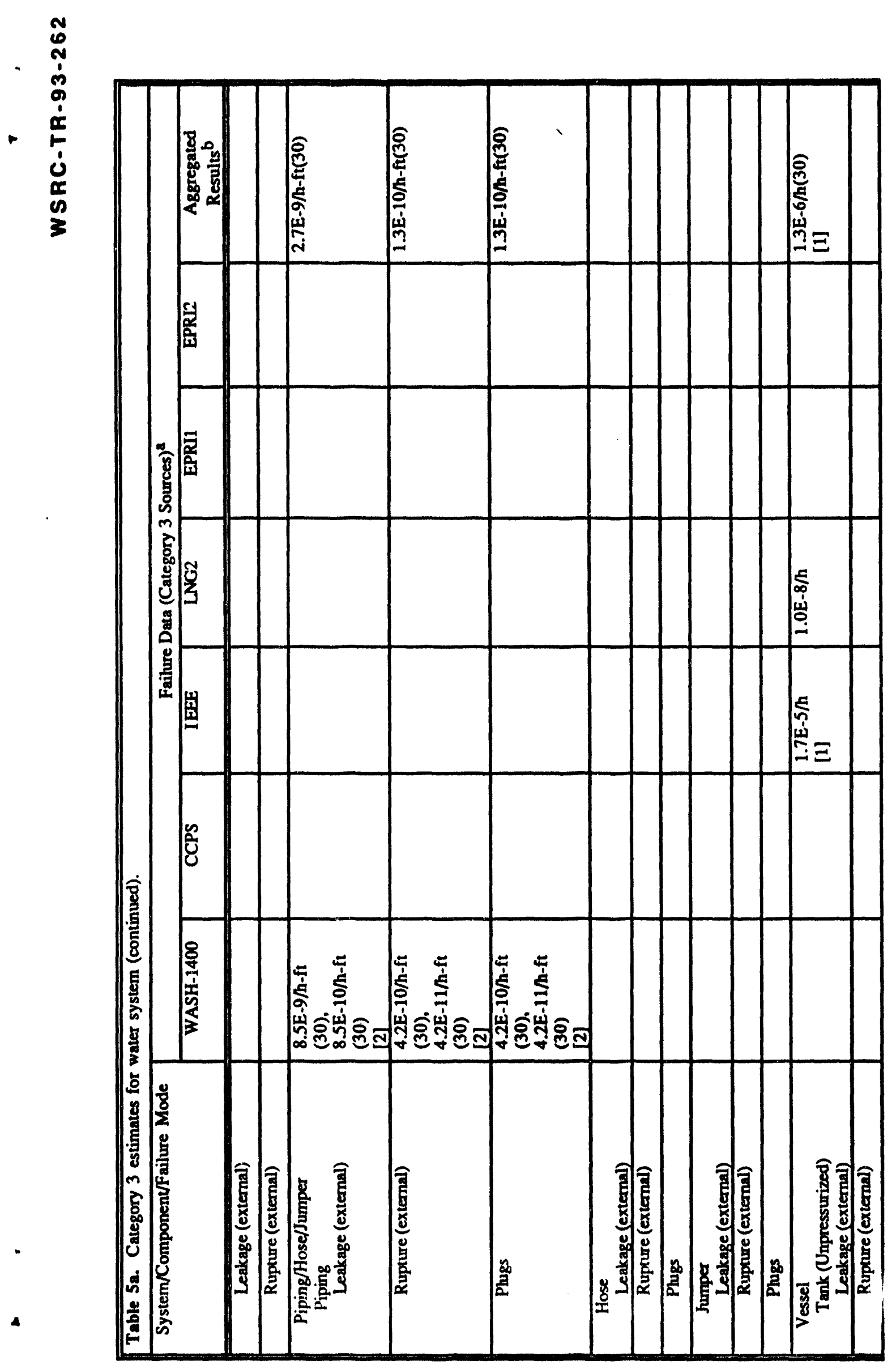

$\cong$ 
WSRC-TR-93-262

\begin{tabular}{|c|c|c|c|c|c|c|c|}
\hline \multirow[t]{2}{*}{ System/Component/Failure Mode } & \multicolumn{7}{|c|}{ Failure Data (Category 3 Sources) ${ }^{2}$} \\
\hline & WASH-1400 & $\overline{\mathrm{CCPS}}$ & IEEE & LNG2 & EPRII & $\overline{\text { EPRI2 }}$ & $\begin{array}{c}\text { Aggregated } \\
\text { Results } \\
\end{array}$ \\
\hline $\begin{array}{l}\text { Tank (Pressurized) } \\
\text { Leakage (external) }\end{array}$ & & & $\begin{array}{l}1.7 \mathrm{E}-6 / \mathrm{h} \\
{[1]}\end{array}$ & & & & $\begin{array}{l}1.3 \mathrm{E}-7 / \mathrm{h}(30) \\
{[3]}\end{array}$ \\
\hline Rupture (external) & & & & & & & \\
\hline \multicolumn{8}{|l|}{$\begin{array}{l}\text { Flange/Gasket } \\
\text { Leakage (extemal) }\end{array}$} \\
\hline \multicolumn{8}{|l|}{ Rupture (external) } \\
\hline $\begin{array}{l}\text { Heat Exchanger } \\
\text { Shell/Tube } \\
\text { Fouling (tubes) }\end{array}$ & & & $\begin{array}{l}6.3 \mathrm{E}-6 / \mathrm{h} \\
{[1,6]}\end{array}$ & & & $\begin{array}{l}1.4 \mathrm{E}-5 / \mathrm{h} \\
{[1]}\end{array}$ & $\begin{array}{l}9.4 \mathrm{E}-6 / \mathrm{h}(10) \\
{[4]}\end{array}$ \\
\hline Plugs (ubbes) & & & {$[6]$} & & & [1] & [4] \\
\hline Leakage (tubes) & & & [6] & & & [1] & [4] \\
\hline \multicolumn{8}{|l|}{ Rupure (nibes) } \\
\hline \multicolumn{8}{|l|}{ Leakage (shell) } \\
\hline \multicolumn{8}{|l|}{ Rupare (shell) } \\
\hline \multicolumn{8}{|l|}{$\begin{array}{l}\text { Heater (Electrical) } \\
\text { Fails to heal }\end{array}$} \\
\hline \multicolumn{8}{|l|}{ Overheats } \\
\hline \multicolumn{8}{|l|}{ Leakage (externa) } \\
\hline \multicolumn{8}{|l|}{ Rupture (external) } \\
\hline $\begin{array}{l}\text { Strainer/Filter } \\
\text { Plugs }\end{array}$ & & & $\begin{array}{l}2.8 \mathrm{E}-6 / \mathrm{h}(3) \\
{[6,7]}\end{array}$ & & & & $\begin{array}{l}2.8 \mathrm{E}-6 / \mathrm{h}(3.0) \\
{[4]}\end{array}$ \\
\hline Leakage (internal) & & & {$[6,7]$} & & & & [4] \\
\hline Rupture (intemal) & & & {$[6,7]$} & & & & [4] \\
\hline $\begin{array}{l}\text { Orifice } \\
\text { Plugs }\end{array}$ & $8.0 \mathrm{E}-7 / \mathrm{h}(3)$ & & & & & & $8.0 \mathrm{E}-7 / \mathrm{h}(3)$ \\
\hline $\begin{array}{l}\text { Miscellaneous } \\
\text { Travelling Screen } \\
\text { Plugs }\end{array}$ & & & & & & & \\
\hline
\end{tabular}

Table 5a. Category 3 estimates for water system.

a. Failure rate estimates are listed as: mean frequency (error factor), where the error factor is the 95 th percentile/50th percentile. Numbers in brackets refer to notes listed below, arranged by source.

b. Aggregated results are mean and error factor (in parentheses). 


\section{Notes}

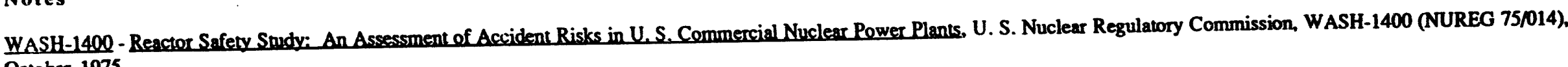
October 1975.

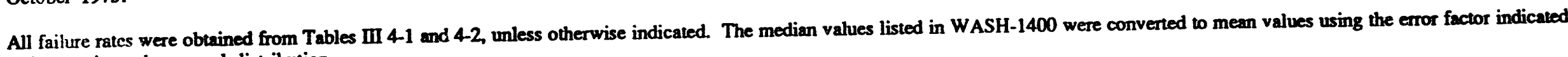
and assuming a lognormal distribution.

1. Equivalent estimate per hour (from air-operated valve information) used.

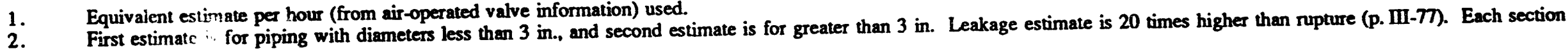
was assumed to be $20 \mathrm{ft}$.

CCPS - Guidelines for Process Equipment Reliability Data With Data Tables, Center for Chemical Process Safety, American Institute of Chemical Engineers, 1989.

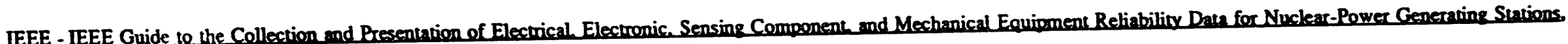
Institute of Electrical and Electronic Engineers, Inc. IEEE Std 500-1984, 1983.

Recommended estimates were interpreted to be medians. Recommended estimates were
unless otherwise indicated. If the error factor was larger than 30, it was rechuced to 30 .

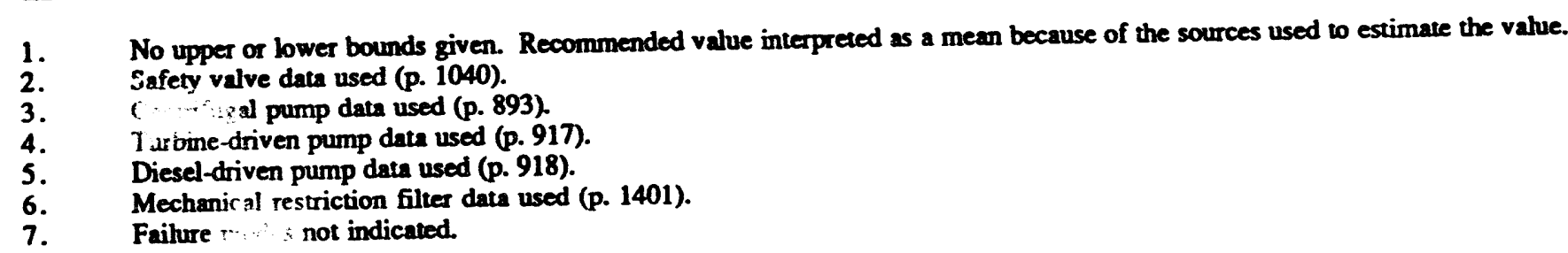

Table 5a. Category 3 estimates for water system.

LNG2 - H. Lammerse and M. Bosman, "Data of Gas Compressors and Instrumentation - Hard to Collect, Easy to Analyse," Reliability Engineering 13 (1985), pp. 65-78.

All estimates were taken from the observed data on p. 78.

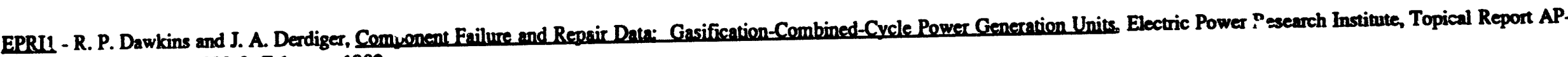
2205, Research Project 239-2, February 1982.

1. Failure modes not indicated. 
EPRI2 - J. A. Derdiger et al., Component Failure and Repair Data for Coal-Fired Power Units, Electric Power Research Institute, Topical Report AP-2071, Research Project 239-2, October 1981.

1. Failure modes not indicated.

\section{Agoregated Results}

1. Aggregation routine resulted in an error factor $>\mathbf{3 0}$, so an error factor of $\mathbf{3 0}$ was assumed.

2. For per hour estiv? :tes without failure mode indicated, one-half was assigned to fails to start and one-half to fails to run.

3. IEEE estimites indicate pressurized tank value is one-tenth of unpressurized value.

4. Failure modes not indicated. 
WSRC-TR-93-262

\begin{tabular}{|c|c|c|c|c|c|c|c|}
\hline \multirow[t]{2}{*}{ System/Component/Failure Mode } & \multicolumn{7}{|c|}{ Failure Estimates (Category 3 Sources) } \\
\hline & WASH-1400 & $\overline{C C P S}$ & IEEE & LNG2 & EPRI1 & EPRI2 & $\begin{array}{l}\text { Aggregated } \\
\text { Results }^{b}\end{array}$ \\
\hline $\begin{array}{l}\text { Chemical Process } \\
\text { Valve (Standby or Safety) } \\
\text { Manual } \\
\text { Fails to open/close }\end{array}$ & & $2.9 \mathrm{E}-4 / \mathrm{d}(8.7)$ & & & & & $2.9 \mathrm{E}-4 / \mathrm{d}(8.7)$ \\
\hline \multicolumn{8}{|l|}{ Plugs } \\
\hline \multicolumn{8}{|l|}{ Leakage (intemal) } \\
\hline \multicolumn{8}{|l|}{ Rupture (internal) } \\
\hline \multicolumn{8}{|l|}{ Leakage (external) } \\
\hline \multicolumn{8}{|l|}{ Rupture (external) } \\
\hline $\begin{array}{l}\text { Check } \\
\text { Fails to open }\end{array}$ & & $1.5 \mathrm{E}-4 / \mathrm{d}(3.2)$ & & & & & $1.5 \mathrm{E}-4 / \mathrm{d}(3.2)$ \\
\hline Fails to close & & $2.2 E-3 / d(4.9)$ & & & & & $2.2 \mathrm{E}-3 / \mathrm{d}(4.9)$ \\
\hline \multicolumn{8}{|l|}{ Plugs } \\
\hline \multicolumn{8}{|l|}{ Leakrge (internal) } \\
\hline \multicolumn{8}{|l|}{ Rupture (internal) } \\
\hline \multicolumn{8}{|l|}{ Leakage (crtemal) } \\
\hline \multicolumn{8}{|l|}{ Rupture (cxlemal) } \\
\hline $\begin{array}{l}\text { Motor-Operased } \\
\text { Fails to oret/close }\end{array}$ & & $5.6 E-3 / d(6.1)$ & & & & & $5.6 \mathrm{E}-3 / \mathrm{d}(6.1)$ \\
\hline Spurious operation & & $1.4 E-6 / h(4.0)$ & & & & & $1.4 \mathrm{E}-6 / \mathrm{h}(4.0)$ \\
\hline \multicolumn{8}{|l|}{ Plugs } \\
\hline \multicolumn{8}{|l|}{ Leakage (inicmal) } \\
\hline \multicolumn{8}{|l|}{ Rupture (intemal) } \\
\hline \multicolumn{8}{|l|}{ Leakage (external) } \\
\hline \multicolumn{8}{|l|}{ Rupture (extcrnal) } \\
\hline $\begin{array}{l}\text { Air-Operated } \\
\text { Fails to orm/close }\end{array}$ & & $2.2 \mathrm{E}-3 / \mathrm{d}(4.7)$ & & $\begin{array}{l}2.4 \mathrm{E}-5 / \mathrm{h} \\
{[1]}\end{array}$ & & & $\begin{array}{l}2.2 \mathrm{E}-3 / \mathrm{d}(4.7) \\
{[1]}\end{array}$ \\
\hline Spurious opreration & & $3.6 \mathrm{E}-6 / \mathrm{h}(6.7)$ & & [1] & & & $\begin{array}{l}3.6 \mathrm{E}-6 / \mathrm{h}(6.7) \\
{[1]}\end{array}$ \\
\hline \multicolumn{8}{|l|}{ Plugs } \\
\hline Leakage (internal) & & & & & & & \\
\hline
\end{tabular}


WSRC-TR-93-262

\begin{tabular}{|c|c|c|c|c|c|c|c|}
\hline \multirow[t]{2}{*}{ System/Component/Failure Mode } & \multicolumn{7}{|c|}{ Failure Estimates (Category 3 Sources) $^{\mathbf{a}}$} \\
\hline & WASH-1400 & CCPS & IEEE & LNG2 & EPRI1 & EPR12 & $\begin{array}{c}\text { Aggregated } \\
\text { Results }^{b} \\
\end{array}$ \\
\hline \multicolumn{8}{|l|}{ Rupture (intcmal) } \\
\hline \multicolumn{8}{|l|}{ Leakage (external) } \\
\hline \multicolumn{8}{|l|}{ Rupture (external) } \\
\hline $\begin{array}{l}\text { Solenoid-Operates. } \\
\text { Fails to open/close }\end{array}$ & & $2.8 \mathrm{E}-3 / \mathrm{d}(5.5)$ & & & & & $2.8 \mathrm{E}-3 / \mathrm{d}(5.5)$ \\
\hline Spurious operation & & $4.1 \mathrm{E}-7 / \mathrm{h}(3.0)$ & & & & & $4.1 \mathrm{E}-7 / \mathrm{h}(3.0)$ \\
\hline \multicolumn{8}{|l|}{ Plugs } \\
\hline \multicolumn{8}{|l|}{ Leakage (internal) } \\
\hline \multicolumn{8}{|l|}{ Rupture (intemal) } \\
\hline \multicolumn{8}{|l|}{ Leakage (external) } \\
\hline \multicolumn{8}{|l|}{ Rupaure (external) } \\
\hline $\begin{array}{l}\text { Safety/Relief } \\
\text { Fails to open }\end{array}$ & & $\begin{array}{l}4.2 \mathrm{E}-3 / \mathrm{d}(30) \text {, } \\
2.1 \mathrm{E}-4 / \mathrm{d}(10)\end{array}$ & & & & & $7.8 \mathrm{E}-4 / \mathrm{d}(18)$ \\
\hline Fails to reclose & & $\begin{array}{l}5.0 \mathrm{E}-3 / \mathrm{d}(10), \\
5.2 \mathrm{E}-3 / \mathrm{d}(13)\end{array}$ & & & & & $5.1 \mathrm{E}-3 / \mathrm{d}(12)$ \\
\hline \multicolumn{8}{|l|}{ Leakage (internal) } \\
\hline \multicolumn{8}{|l|}{ Rupture (internal) } \\
\hline \multicolumn{8}{|l|}{ Leakage (external) } \\
\hline \multicolumn{8}{|l|}{ Rupture (extemal) } \\
\hline \multicolumn{8}{|l|}{$\begin{array}{c}\text { Vacuum-Breaker } \\
\text { Fails to open }\end{array}$} \\
\hline \multicolumn{8}{|l|}{ Fails to reclose } \\
\hline \multicolumn{8}{|l|}{ Leakage (internal) } \\
\hline \multicolumn{8}{|l|}{ Rupture (internal) } \\
\hline \multicolumn{8}{|l|}{ Leakage (external) } \\
\hline \multicolumn{8}{|l|}{ Rupture (external) } \\
\hline \multicolumn{8}{|l|}{$\begin{array}{c}\text { Valve (Control) } \\
\text { Motor-Operated } \\
\text { Fails open } \\
\end{array}$} \\
\hline Fails closed & & & & & & & \\
\hline Fails in respond & & & & & & & \\
\hline
\end{tabular}




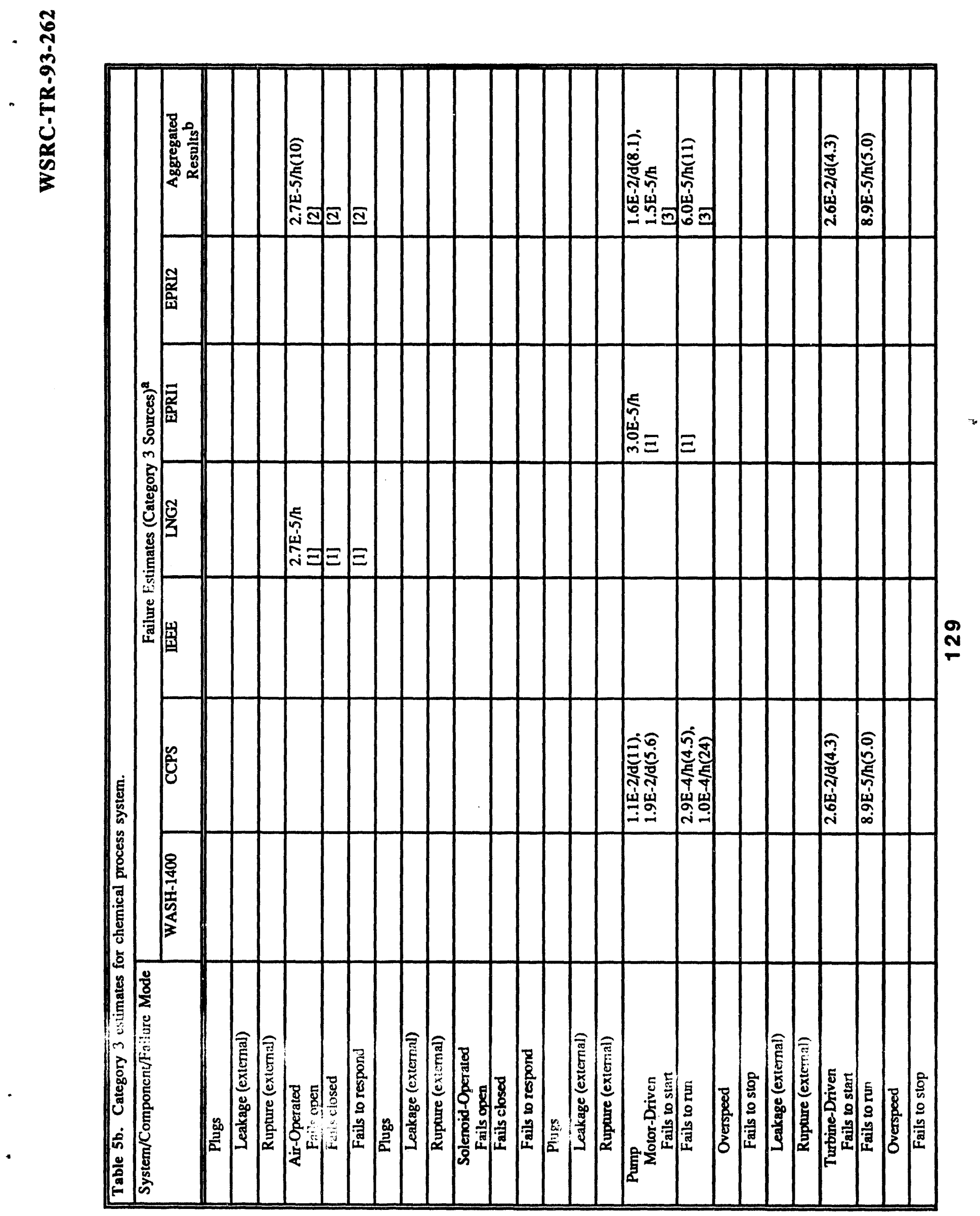


WSRC-TR-93-262

\begin{tabular}{|c|c|c|c|c|c|c|c|}
\hline \multirow[t]{2}{*}{ System/Component/Failure Mode } & \multicolumn{7}{|c|}{ Failure Estimates (Category 3 Sources) ${ }^{\mathrm{a}}$} \\
\hline & WASH-1400 & CCPS & IEEE & LNG2 & EPRII & EPRI2 & $\begin{array}{c}\text { Aggregated } \\
\text { Results }^{\mathrm{b}} \\
\end{array}$ \\
\hline \multicolumn{8}{|l|}{ Leakage (external) } \\
\hline \multicolumn{8}{|l|}{ Rupture (external) } \\
\hline \multicolumn{8}{|l|}{$\begin{array}{l}\text { Diesel-Driven } \\
\text { Fails to start }\end{array}$} \\
\hline \multicolumn{8}{|l|}{ Fails to run } \\
\hline \multicolumn{8}{|l|}{ Overspeed } \\
\hline \multicolumn{8}{|l|}{ Fails to stop } \\
\hline \multicolumn{8}{|l|}{ Leakage (external) } \\
\hline \multicolumn{8}{|l|}{ Rupture (extemal) } \\
\hline \multicolumn{8}{|l|}{$\begin{array}{l}\text { Piping/Hose/Jumper } \\
\text { Piping } \\
\text { Leakage (external) }\end{array}$} \\
\hline Rupture (extemsl) & & $\begin{array}{l}5.1 \mathrm{E}-12 / \mathrm{h}-\mathrm{ft} \\
\text { (15) } \\
{[1]}\end{array}$ & & & & & $5.1 \mathrm{E}-12 / \mathrm{h}-\mathrm{ft}(15)$ \\
\hline \multicolumn{8}{|l|}{ Plugs } \\
\hline \multicolumn{8}{|l|}{$\begin{array}{l}\text { Hose } \\
\text { Leakage (external) }\end{array}$} \\
\hline Rupture (extemal) & & $\begin{array}{l}2.9 \mathrm{E}-8 / \mathrm{h}-\mathrm{ft} \\
(15) \\
{[1]}\end{array}$ & & & & & $2.9 \mathrm{E}-8 / \mathrm{h}-\mathrm{ft}(15)$ \\
\hline \multicolumn{8}{|l|}{ Plugs } \\
\hline \multicolumn{8}{|l|}{$\begin{array}{l}\text { Jumper } \\
\text { Leakage (external) }\end{array}$} \\
\hline \multicolumn{8}{|l|}{$\begin{array}{l}\text { Leakage (external) } \\
\text { Rupture (external) }\end{array}$} \\
\hline \multicolumn{8}{|l|}{ Plugs } \\
\hline $\begin{array}{l}\text { Vessel } \\
\text { Tank (Unpressurized) } \\
\text { Leakage (external) } \\
\end{array}$ & & $\begin{array}{l}9.9 \mathrm{E}-7 / \mathrm{h}(4.9) \\
1.2 \mathrm{E}-6 / \mathrm{h}(15)\end{array}$ & & & & & $1.2 \mathrm{E}-6 / \mathrm{h}(9.3)$ \\
\hline \multicolumn{8}{|l|}{ Rupture (external) } \\
\hline $\begin{array}{l}\text { Tank (Pressurized) } \\
\text { Leakage (extemal) }\end{array}$ & & $1.1 \mathrm{E}-8 / \mathrm{h}(17)$ & & & & & $1.1 \mathrm{E}-8 / \mathrm{h}(17)$ \\
\hline Rupture (extermal) & & & & & & & \\
\hline
\end{tabular}


WSRC-TR-93-262

\begin{tabular}{|c|c|c|c|c|c|c|c|}
\hline \multirow[t]{2}{*}{ System/Component/Failure Mode } & \multicolumn{7}{|c|}{ Failure Estimates (Category 3 Sources) ${ }^{\mathrm{a}}$} \\
\hline & WASH-1400 & CCPS & IEEE & LNG2 & EPRII & EPRI2 & $\begin{array}{l}\text { Aggregated } \\
\text { Results }^{b}\end{array}$ \\
\hline \multicolumn{8}{|l|}{ Flange/Gasket } \\
\hline \multicolumn{8}{|l|}{ Kupturc (extemal) } \\
\hline \multicolumn{8}{|l|}{$\begin{array}{l}\text { Heat Exchanger } \\
\text { Shell/Tube } \\
\text { Fouling (tubes) }\end{array}$} \\
\hline \multicolumn{8}{|l|}{ Plugs (tubes) } \\
\hline Leakage (tubes) & & $2.6 \mathrm{E}-5 / \mathrm{h}(7.5)$ & & & & & $2.6 \mathrm{E}-5 / \mathrm{h}(7.5)$ \\
\hline \multicolumn{8}{|l|}{ Rupture (tuibes) } \\
\hline \multicolumn{8}{|l|}{ Leakage (shell) } \\
\hline \multicolumn{8}{|l|}{ Rupture (shell) } \\
\hline \multicolumn{8}{|l|}{$\begin{array}{l}\text { Heater (Electrical) } \\
\text { Fails to heat }\end{array}$} \\
\hline \multicolumn{8}{|l|}{ Overheats } \\
\hline \multicolumn{8}{|l|}{ Leakage (extemal) } \\
\hline \multicolumn{8}{|l|}{ Rupture (extemal) } \\
\hline $\begin{array}{l}\text { Strainer/Filter } \\
\text { Plugs }\end{array}$ & & & $\begin{array}{l}5.6 \mathrm{E}-6 / \mathrm{h}(5.0) \\
{[1,2]}\end{array}$ & & & & $\begin{array}{l}5.6 \mathrm{E}-6 / \mathrm{h}(5.0) \\
{[2]}\end{array}$ \\
\hline Leakage (intemal) & & & [2] & & & & [2] \\
\hline Rupture (internal) & & & [2] & & & & [2] \\
\hline \multicolumn{8}{|l|}{$\begin{array}{c}\text { Orifice } \\
\text { Plugs }\end{array}$} \\
\hline \multicolumn{8}{|l|}{$\begin{array}{l}\text { Miscellaneous } \\
\text { Mixer/Blender } \\
\text { Failure }\end{array}$} \\
\hline $\begin{array}{l}\text { Agitator } \\
\text { Failure }\end{array}$ & & & & & $5.7 \mathrm{E}-5 / \mathrm{h}$ & $\begin{array}{l}1.0 \mathrm{E}-5 / \mathrm{h}, \\
1.9 \mathrm{E}-5 / \mathrm{h}, \\
3.0 \mathrm{E}-6 / \mathrm{h}, \\
7.0 \mathrm{E}-6 / \mathrm{h}, \\
3.8 \mathrm{E}-5 / \mathrm{h}\end{array}$ & $2.5 \mathrm{E}-5 / \mathrm{h}(10)$ \\
\hline $\begin{array}{l}\text { Centrifuge } \\
\text { Faihure }\end{array}$ & & & & & & & \\
\hline
\end{tabular}


Table 5b. Category 3 estimates for chemical process system.

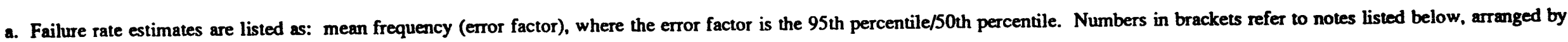
source.

b. Aggregated results are mean and error factor (in parentheses).

Notes

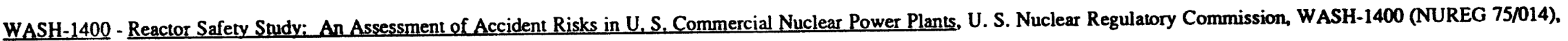
October 1975.

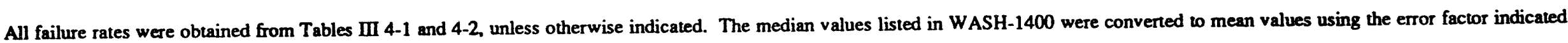
and assuming a lognormal distribution.

CCPS - Guidelines for Process Equipment Reliability Data With Data Tables, Center for Chemical Process Safety. American Institute of Criemical Engineers, 1989.

Error factor estimates were obtrined from the square root of the upper bound times the lower bound.

1. Each section was assumed to be $20 \mathrm{ft}$.

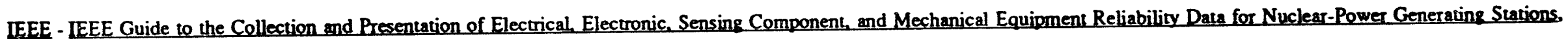
Institute of Electrical and Electronic Engineers. Inc.. IEEE Std 500-1984, 1983.

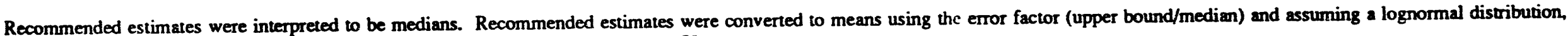
unless otherwise indicated. If the error factor was larger than 50 , it was reduced to 50.

1. Strainer/filter for chemical fluid estimate used (p. 1407).

2. Failure modes not indicated.

LNG2 - H. Lammerse and M. Bosman, "Data of Gas Compressors and Instrumentation - Hard to Collect, Easy to Analyse," Reliability Enpineering 13 (1985), Pp. 65-78.

All estimates were taken from the observed dats on p. 78.

1. Failure modes not indicated. 
EPRIl - R. P. Dawkins and J. A. Derdiger, Component Failure and Repair Data; Gasification-Combined-Cycle Power Generation Units, Electric Power Reseanch Institute, Topical Report AP2205, Research Project 239-2, February 1982.

1. Faikure modes not indicated.

Table 5b. Category 3 estimates for chemical process system.

EPRI2 - J. A. Derdiger et al., Component Failure and Repair Data for Coal-Fired Power Units, Electric Power Research Instiute, Topical Report AP-2071. Research Project 239-2, October 1981.

\section{Asgregated Results}

1. LNG2 data not used (no information on how to partition among failure modes).

2. Failure modes not indicated.

3. Per hour estimate without failure modes indicated was divided evenly between fails to start and fails to run. 
WSRC-TR-93-262

\begin{tabular}{|c|c|c|c|c|c|c|c|}
\hline \multirow[t]{2}{*}{ System/Component/Failure Mode } & \multicolumn{7}{|c|}{ Failure Estimates (Category 3 Sources) } \\
\hline & WASH-1400 & CCPS & IEEE & LNG2 & EPRI1 & EPR12 & $\begin{array}{l}\text { Aggregated } \\
\text { Results }^{b}\end{array}$ \\
\hline \multicolumn{8}{|l|}{$\begin{array}{l}\text { Compressed Gas } \\
\text { Vilve (Standby or Safety) } \\
\text { Manual } \\
\text { Fails to open/close } \\
\end{array}$} \\
\hline \multicolumn{8}{|l|}{ Plugs } \\
\hline \multicolumn{8}{|l|}{ Leakage (internal) } \\
\hline \multicolumn{8}{|l|}{ Rupture (intemal) } \\
\hline \multicolumn{8}{|l|}{ Leakage (extemal) } \\
\hline \multicolumn{8}{|l|}{ Rupture (external) } \\
\hline \multicolumn{8}{|l|}{$\begin{array}{l}\text { Check } \\
\text { Fails to open }\end{array}$} \\
\hline \multicolumn{8}{|l|}{ Fails to close } \\
\hline \multicolumn{8}{|l|}{ Plugs } \\
\hline \multicolumn{8}{|l|}{ Leakage (mtema) } \\
\hline \multicolumn{8}{|l|}{ Ruppure (internal) } \\
\hline \multicolumn{8}{|l|}{ Leakage (extemal) } \\
\hline \multicolumn{8}{|l|}{ Rupurre (external) } \\
\hline \multicolumn{8}{|l|}{$\begin{array}{l}\text { Motor-Operated } \\
\text { Fails to open/close }\end{array}$} \\
\hline \multicolumn{8}{|l|}{ Spurious operation } \\
\hline \multicolumn{8}{|l|}{ Plugs } \\
\hline \multicolumn{8}{|l|}{ Leakage (intemal) } \\
\hline \multicolumn{8}{|l|}{ Rupture (internal) } \\
\hline \multicolumn{8}{|l|}{ Leakage (external) } \\
\hline \multicolumn{8}{|l|}{ Rupture (external) } \\
\hline \multicolumn{8}{|l|}{$\begin{array}{l}\text { Air-Operated } \\
\text { Fails to open/close }\end{array}$} \\
\hline \multicolumn{8}{|l|}{ Spurious operation } \\
\hline \multicolumn{8}{|l|}{ Plugs } \\
\hline Leakage (intemal) & & & & & & & \\
\hline
\end{tabular}


WSRC-TR-93-262

\begin{tabular}{|c|c|c|c|c|c|c|c|}
\hline \multirow[t]{2}{*}{ System/Componen//Failure Mode } & \multicolumn{7}{|c|}{ Failure Estimates (Category 3 Sources) } \\
\hline & WASH-1400 & CCPS & IEEE & LNG2 & EPRII & EPRI2 & $\begin{array}{l}\text { Aggregated } \\
\text { Results } b\end{array}$ \\
\hline \multicolumn{8}{|l|}{ Rupture (internal) } \\
\hline \multicolumn{8}{|l|}{ Leakage (exicmal) } \\
\hline \multicolumn{8}{|l|}{ Rupture (extemal) } \\
\hline \multicolumn{8}{|l|}{$\begin{array}{l}\text { Solenoi: sersted } \\
\text { Fails to open/close }\end{array}$} \\
\hline \multicolumn{8}{|l|}{ Spurious operation } \\
\hline \multicolumn{8}{|l|}{ Plugs } \\
\hline \multicolumn{8}{|l|}{ Leakace (intemal) } \\
\hline \multicolumn{8}{|l|}{ Rupture (internal) } \\
\hline \multicolumn{8}{|l|}{ Leakage (extermal) } \\
\hline \multicolumn{8}{|l|}{ Rupurre (exiernal) } \\
\hline \multicolumn{8}{|l|}{$\begin{array}{l}\text { Safety/Relief } \\
\text { Fails to open }\end{array}$} \\
\hline \multicolumn{8}{|l|}{ Fails w: reclose } \\
\hline \multicolumn{8}{|l|}{ Leakage (internal) } \\
\hline \multicolumn{8}{|l|}{ Rupture (intemal) } \\
\hline \multicolumn{8}{|l|}{ Leakage (external) } \\
\hline \multicolumn{8}{|l|}{ Rupbure (external) } \\
\hline \multicolumn{8}{|l|}{$\begin{array}{l}\text { Vacuum-B - iker } \\
\text { Fails to open }\end{array}$} \\
\hline \multicolumn{8}{|l|}{ Fails to reclose } \\
\hline \multicolumn{8}{|l|}{ Leakage (internal) } \\
\hline \multicolumn{8}{|l|}{ Rupture (intermal) } \\
\hline \multicolumn{8}{|l|}{ Leakage (extemal) } \\
\hline \multicolumn{8}{|l|}{ Ruphure (external) } \\
\hline \multicolumn{8}{|l|}{$\begin{array}{l}\text { Valve (Control) } \\
\text { Motor-Operated } \\
\text { Fails open } \\
\end{array}$} \\
\hline Fails closed & & & & & & & \\
\hline
\end{tabular}

135 
WSRC-TR-93-262

\begin{tabular}{|c|c|c|c|c|c|c|c|}
\hline \multirow[t]{2}{*}{ System/Component/Failure Mode } & \multicolumn{7}{|c|}{ Failure Estimates (Category 3 Sources) ${ }^{\mathrm{a}}$} \\
\hline & WASH-1400 & CCPS & IEEE & LNG2 & EPRII & EPRI2 & $\begin{array}{l}\text { Aggrega:ed } \\
\text { Results }{ }^{b}\end{array}$ \\
\hline \multicolumn{8}{|l|}{ Fails to respond } \\
\hline \multicolumn{8}{|l|}{ Plugs } \\
\hline \multicolumn{8}{|l|}{ Leakage (external) } \\
\hline \multicolumn{8}{|l|}{ Rupture (external) } \\
\hline \multicolumn{8}{|l|}{$\begin{array}{l}\text { Air-Operated } \\
\text { Fails open }\end{array}$} \\
\hline \multicolumn{8}{|l|}{ Fails closed } \\
\hline \multicolumn{8}{|l|}{ Fails to respond } \\
\hline \multicolumn{8}{|l|}{ Plugs } \\
\hline \multicolumn{8}{|l|}{ Leakage (external) } \\
\hline \multicolumn{8}{|l|}{ Rupture (extermal) } \\
\hline \multicolumn{8}{|l|}{$\begin{array}{l}\text { Solenoid-Operated } \\
\text { Fails open }\end{array}$} \\
\hline \multicolumn{8}{|l|}{ Fails closed } \\
\hline \multicolumn{8}{|l|}{ Fails to respond } \\
\hline \multicolumn{8}{|l|}{ Plugs } \\
\hline \multicolumn{8}{|l|}{ Leakage (extemal) } \\
\hline \multicolumn{8}{|l|}{ Rupture (extemal) } \\
\hline $\begin{array}{l}\text { Compressor } \\
\text { Motor-Driven } \\
\text { Fails to start }\end{array}$ & & & $\begin{array}{l}6.7 \mathrm{E}-4 / \mathrm{h}(30) \\
4.7 \mathrm{E}-5 / \mathrm{h} \\
\text { [1] }\end{array}$ & & $\begin{array}{l}3.8 \mathrm{E}-5 / \mathrm{h} \\
{[1]}\end{array}$ & & $\begin{array}{l}1.6 \mathrm{E}-4 / \mathrm{h}(30) \\
{[1.2]}\end{array}$ \\
\hline Fails to run & & $2.5 \mathrm{E}-3 / \mathrm{h}(19)$ & [1] & & [1] & & [2] \\
\hline \multicolumn{8}{|l|}{ Overspeed } \\
\hline \multicolumn{8}{|l|}{ Fails to stop } \\
\hline \multicolumn{8}{|l|}{ Leakage (extermal) } \\
\hline \multicolumn{8}{|l|}{ Rupture (external) } \\
\hline \multicolumn{8}{|l|}{$\begin{array}{l}\text { Piping/Hose/Jumper/Tube } \\
\text { Piping } \\
\text { Leakage (external) }\end{array}$} \\
\hline Rupture (extemal) & & & & & & & \\
\hline
\end{tabular}


WSRC-TR-93-262

\begin{tabular}{|c|c|c|c|c|c|c|c|}
\hline \multirow[t]{2}{*}{ System/Componen/Frilure Mode } & \multicolumn{7}{|c|}{ Failure Estimates (Category 3 Sources) } \\
\hline & WASH-1400 & CCPS & IEEE & LNG2 & EPRI1 & EPR12 & $\begin{array}{l}\text { Aggregated } \\
\text { Results }^{b}\end{array}$ \\
\hline \multicolumn{8}{|l|}{ Plugs } \\
\hline \multicolumn{8}{|l|}{$\begin{array}{l}\text { Hose } \\
\text { Leakage (extemal) }\end{array}$} \\
\hline \multicolumn{8}{|l|}{ Rupture (external) } \\
\hline \multicolumn{8}{|l|}{ Plugs } \\
\hline \multicolumn{8}{|l|}{$\begin{array}{l}\text { Jumper } \\
\text { Leakage (external) }\end{array}$} \\
\hline \multicolumn{8}{|l|}{ Rupture (external) } \\
\hline \multicolumn{8}{|l|}{ Plugs } \\
\hline \multicolumn{8}{|l|}{$\begin{array}{l}\text { Tube } \\
\text { Leakage (extemal) }\end{array}$} \\
\hline \multicolumn{8}{|l|}{ Rupture (externs) } \\
\hline \multicolumn{8}{|l|}{ Plugs } \\
\hline \multicolumn{8}{|l|}{$\begin{array}{l}\text { Vessel } \\
\text { Tank (Pressurizod) } \\
\text { Leakage (external) }\end{array}$} \\
\hline \multicolumn{8}{|l|}{ Rupare (extemal) } \\
\hline \multicolumn{8}{|l|}{$\begin{array}{l}\text { Cylinder (Pressurized) } \\
\text { Leakage (extermal) }\end{array}$} \\
\hline \multicolumn{8}{|l|}{ Rupture (external) } \\
\hline \multicolumn{8}{|l|}{$\begin{array}{l}\text { Flange/Gasket } \\
\text { Leakage (externa) }\end{array}$} \\
\hline \multicolumn{8}{|l|}{ Rupture (external) } \\
\hline \multicolumn{8}{|l|}{$\begin{array}{l}\text { Heat Exchanger } \\
\text { Shell/Tube } \\
\text { Fouling (ubes) }\end{array}$} \\
\hline \multicolumn{8}{|l|}{ Plugs (ubbes) } \\
\hline \multicolumn{8}{|l|}{ Leakage (ubbes) } \\
\hline \multicolumn{8}{|l|}{ Rupture (tubes) } \\
\hline \multicolumn{8}{|l|}{ Leakage (shell) } \\
\hline Rupture (shell) & & & & & & & \\
\hline
\end{tabular}


WSRC-TR-93-262

\begin{tabular}{|c|c|c|c|c|c|c|c|}
\hline \multirow[t]{2}{*}{ System/Component/Failure Mode } & \multicolumn{7}{|c|}{ Failure Estimates (Category 3 Sources) $^{\mathrm{a}}$} \\
\hline & WASH-1400 & CCPS & IEEE & LNG2 & EPRII & EPR12 & $\begin{array}{l}\text { Aggregated } \\
\text { Results }\end{array}$ \\
\hline \multicolumn{8}{|l|}{$\begin{array}{l}\text { Heater (Electrical) } \\
\text { Fails to heat }\end{array}$} \\
\hline \multicolumn{8}{|l|}{ Overheats } \\
\hline \multicolumn{8}{|l|}{ Leakage (external) } \\
\hline \multicolumn{8}{|l|}{ Rupture (external) } \\
\hline \multicolumn{8}{|l|}{$\begin{array}{c}\text { Vaporizer } \\
\text { Failure }\end{array}$} \\
\hline $\begin{array}{c}\text { Air Dryer } \\
\text { Failure } \\
\end{array}$ & & & $\begin{array}{l}2.6 \mathrm{E}-7 / \mathrm{h} \\
{[2]}\end{array}$ & & & & $\begin{array}{l}2.6 \mathrm{E}-7 / \mathrm{h}(10) \\
{[3]}\end{array}$ \\
\hline $\begin{array}{c}\text { Filter } \\
\text { Plugs }\end{array}$ & & & $\begin{array}{l}3.4 \mathrm{E}-6 / \mathrm{h}(3.4) \\
{[1]}\end{array}$ & & & & $\begin{array}{l}3.4 \mathrm{E}-6 / \mathrm{h}(3.4) \\
{[2]}\end{array}$ \\
\hline Leakage (internal) & & & [1] & & & & [2] \\
\hline Rupare (internal) & & & [1] & & & & [2] \\
\hline $\begin{array}{c}\text { Orifice } \\
\text { Plugs }\end{array}$ & & & & & & & \\
\hline
\end{tabular}

Table 5c. Category 3 estimates for compressed gas system.

2. Failure rate estimates are listed as: mean frequency (enror factor), where the error factor is the 95th percentile/50th percentile. Numbers in brackets refer to notes listed below, arranged by source.

b. Aggregated results are mean and error factor (in parentheses).

Notes

WASH-1400 - Reactor Safety Study: An Assessment of Accident Risks in U. S. Commercial Nuclear Power Plants, U. S. Nuclear Regulatory Commission, WASH-1400 (NUREG 75/014), October 1975.

All failure rates were obtained from Tables III 4-1 and 4-2, unless otherwise indicated. The median values listed in WASH-1400 were converted to mean values using the error factor indicated and assuming a lognormal distribution. 
CCPS - Guidelines for Process Equipment Relinbility Data With Data Tables, Center for Chemical Process Safety, American Institute of Chemical Engineers, 1989.

Error factor estimates were obtained from the square root of the upper bound times the lower bound.

IEEE - IEEE Guide to the Collection and Presentation of Electrical Electronic. Sensine Components and Mecianical Equipment Reliability Data for Nuclear-Power Generatine Stations. Institute of Electrical and Electronic Engineers, Inc., IEEE Std 500-1984, 1983.

Recommended estimates were interpreted to be medians. Recommended estimates were converted to means using the error factor (upper bound/median) and assuming a lognormal distribution. unless otherwise indicated. If the error factor was larger than $\mathbf{5 0}$, it was reduced to $\mathbf{5 0}$

1. Failure modes not indicated.

2. Air dryer estimate obtained from information on p. 1422 (no failures in $1.9 \mathrm{E}+6 \mathrm{~h}$ ).

LNG2 - H. Lammerse and M. Bosman, "Data of Gas Compressors and Instrumentation - Hard to Collect, Easy to Analyse," Reliability Engineering 13 (1985), pp. 65-78.

All estimates were taken from the observed data on p. 78.

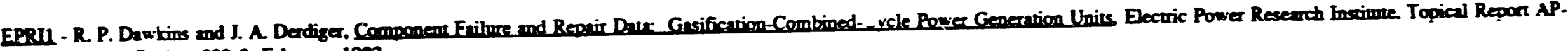
205. Research Project 239-2. Februmy 1982

1. Failure modes not indicated.

EPR12 - J. A. Derdiger a al, Componeme Fuilure and Repair Data for Coal-Fired Power Units Electric Power Research Instiwte, Topical Repon AP-2071, Research Project 229-2 October 1981.

Table 5c. Category 3 estimates for compressed gas system.

\section{Asererated Results}

1. CCPS estimate doubled (assumes equal failure rate estimates for fails to start and fails to run) before combining with other estimates.

2. Faihure modes not indicated.

3. Failure rate estimate based on zero faihures and may be conservatively high. 
WSRC-TR-93-262

\begin{tabular}{|c|c|c|c|c|c|c|c|}
\hline \multirow[t]{2}{*}{ System/Component/Failure Mode } & \multicolumn{7}{|c|}{ Failure Estimates (Category 3 Sources) ${ }^{a}$} \\
\hline & WASH-1400 & CCPS & $\overline{\text { EEEE }}$ & LNG2 & EPRI1 & EPRI2 & $\begin{array}{c}\text { Aggregated } \\
\text { Results }^{b}\end{array}$ \\
\hline \multicolumn{8}{|l|}{$\begin{array}{l}\text { HVAC/Exhaust } \\
\text { Damper (Standby or Safety) } \\
\text { Manual } \\
\text { Fails to open/close } \\
\end{array}$} \\
\hline \multicolumn{8}{|l|}{ Plugs } \\
\hline \multicolumn{8}{|l|}{ Leakage (intemal) } \\
\hline \multicolumn{8}{|l|}{ Rupture (internal) } \\
\hline \multicolumn{8}{|l|}{ Leakage (external) } \\
\hline \multicolumn{8}{|l|}{ Rupture (external) } \\
\hline \multicolumn{8}{|l|}{$\begin{array}{l}\text { Motor-Operated } \\
\text { Fails to open/close }\end{array}$} \\
\hline \multicolumn{8}{|l|}{ Spurious operation } \\
\hline \multicolumn{8}{|l|}{ Plugs } \\
\hline \multicolumn{8}{|l|}{ Leakage (intemal) } \\
\hline \multicolumn{8}{|l|}{ Rupture (internal) } \\
\hline \multicolumn{8}{|l|}{ Leakage (external) } \\
\hline \multicolumn{8}{|l|}{ Rupture (external) } \\
\hline $\begin{array}{l}\text { Air-Operated } \\
\text { Fails to open/close }\end{array}$ & & & $\begin{array}{l}1.2 \mathrm{E}-6 / \mathrm{h} \\
{[1,2]}\end{array}$ & & & & $\begin{array}{l}1.2 \mathrm{E}-6 / \mathrm{h}(10) \\
{[1]}\end{array}$ \\
\hline Spurious operation & & & [1] & & & & [1] \\
\hline \multicolumn{8}{|l|}{ Plugs } \\
\hline \multicolumn{8}{|l|}{ Leakage (intemal) } \\
\hline \multicolumn{8}{|l|}{ Rupture (intemal) } \\
\hline \multicolumn{8}{|l|}{ Leakage (external) } \\
\hline \multicolumn{8}{|l|}{ Rupture (external) } \\
\hline \multicolumn{8}{|l|}{$\begin{array}{c}\text { Damper (Control) } \\
\text { Motor-Operatcd } \\
\text { Fails open }\end{array}$} \\
\hline \multicolumn{8}{|l|}{ Fails closed } \\
\hline \multicolumn{8}{|l|}{ Fails to respond } \\
\hline \multicolumn{8}{|l|}{ Plugs } \\
\hline Leakage (external) & & & & & & & \\
\hline
\end{tabular}


WSRC-TR-93-262

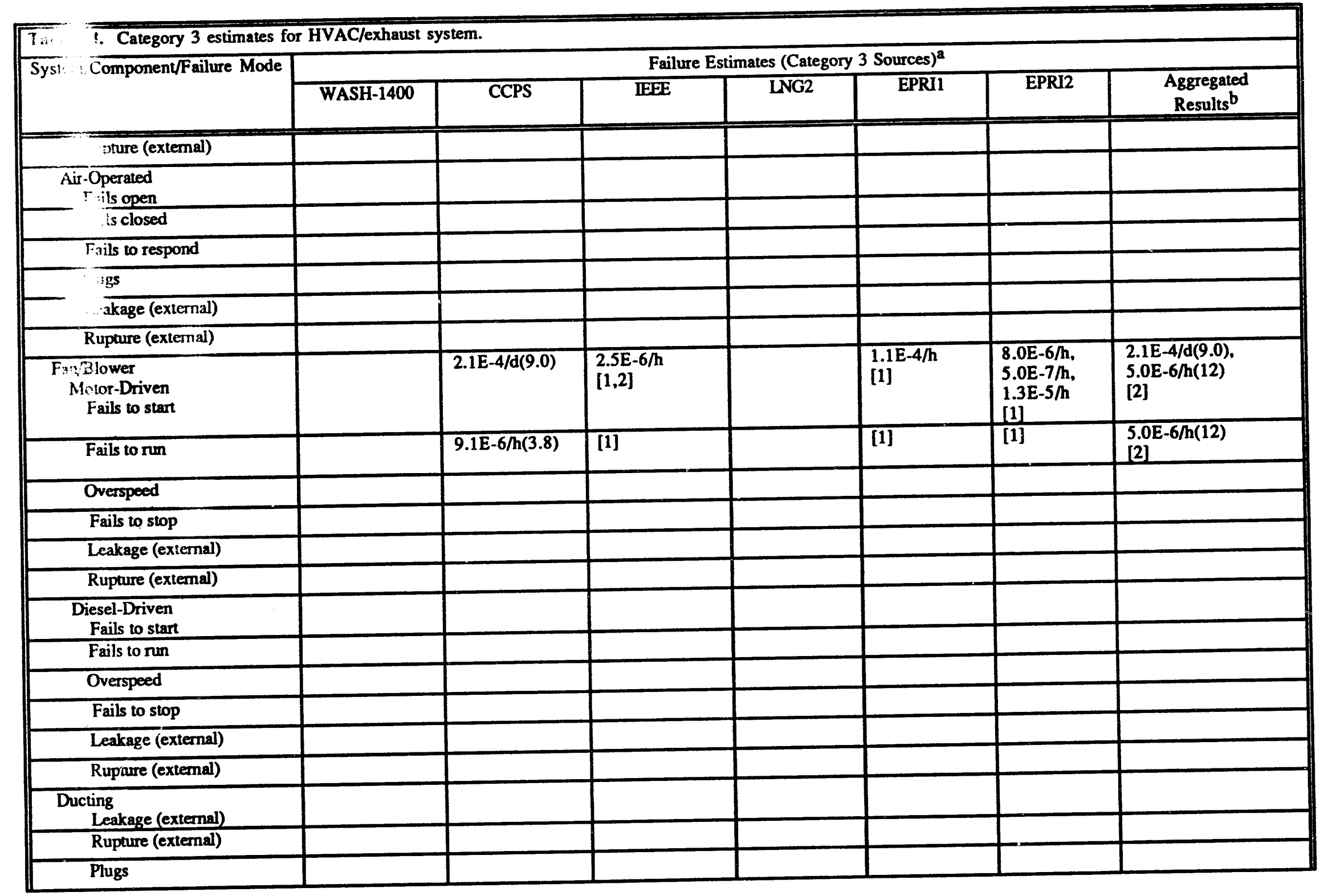


WSRC-TR-93-262

\begin{tabular}{|c|c|c|c|c|c|c|c|}
\hline \multirow[t]{2}{*}{ System/Component/Failure Mode } & \multicolumn{7}{|c|}{ Failure Estimates (Category 3 Sources) ${ }^{\mathrm{a}}$} \\
\hline & WASH-1400 & CCPS & IEEE & LNG2 & EPRI1 & EPRI2 & $\begin{array}{c}\text { Aggregated } \\
\text { Results }\end{array}$ \\
\hline $\begin{array}{l}\text { Heat Exchanger } \\
\text { Air Conditioning Unit/ } \\
\text { Chiller } \\
\text { Fails to start }\end{array}$ & & & $\begin{array}{l}1.0 \mathrm{E}-5 / \mathrm{h}(9.7) \\
{[1,3]}\end{array}$ & & & & $\begin{array}{l}1.0 \mathrm{E}-5 / \mathrm{h}(9.7) \\
{[1]}\end{array}$ \\
\hline Fails to run & & & [1] & & & & [1] \\
\hline $\begin{array}{l}\text { Fan Cooler Unit } \\
\text { Fails to start }\end{array}$ & & & & & & & \\
\hline Fails to run & & & & & & & \\
\hline $\begin{array}{l}\text { Heater (Electrical) } \\
\text { Fails to heat }\end{array}$ & & & & & & & \\
\hline Overheats & & & & & & & \\
\hline $\begin{array}{l}\text { Heater (Gas) } \\
\text { Fails to heat }\end{array}$ & & & & & & & \\
\hline Overheats & & & & & & & \\
\hline $\begin{array}{l}\text { Filter } \\
\text { Normal } \\
\text { Plugs }\end{array}$ & & & & & & & \\
\hline Leakage (internal) & & & & & & & \\
\hline Rupture (intemal) & & & & & & & \\
\hline $\begin{array}{l}\text { Low-Efficiency } \\
\text { Plugs }\end{array}$ & & & & & & & \\
\hline Leakage (internal) & & & & & & & \\
\hline Rupture (internal) & & & & & & & \\
\hline $\begin{array}{l}\text { HEPA } \\
\text { Plugs } \\
\end{array}$ & & & & & & & \\
\hline Leakage (intemal) & & & & & & & \\
\hline Rupture (internal) & & & & & & & \\
\hline $\begin{array}{l}\text { Sand } \\
\text { Plugs } \\
\end{array}$ & & & & & & & \\
\hline Leakage (internal) & & & & & & & \\
\hline Rupture (internal) & & & & & & & \\
\hline $\begin{array}{c}\text { Baghouse } \\
\text { Plugs }\end{array}$ & & & & & & $8.6 \mathrm{E}-4 / \mathrm{h}$ & $8.6 \mathrm{E}-4 / \mathrm{h}(10)$ \\
\hline Leakage (internal) & & & & & & & \\
\hline
\end{tabular}


WSRC-TR-93-262

\begin{tabular}{|c|c|c|c|c|c|c|c|}
\hline \multirow[t]{2}{*}{ System/Componen/Failure Mode } & \multicolumn{7}{|c|}{ Failure Estimates (Category 3 Sources) } \\
\hline & WASH-1400 & CCPS & IEEE & $\overline{\text { LNG2 }}$ & EPRI1 & EPR12 & $\begin{array}{l}\text { Aggregated } \\
\text { Results }\end{array}$ \\
\hline \multicolumn{8}{|l|}{ Rupture (internal) } \\
\hline $\begin{array}{l}\text { Miscellaneous } \\
\text { Mist Eliminator } \\
\text { Failure }\end{array}$ & & & & & & $1.1 \mathrm{E}-4 / \mathrm{h}$ & $1.1 \mathrm{E}-4 / \mathrm{h}(10)$ \\
\hline $\begin{array}{c}\text { Scrubber } \\
\text { Failure }\end{array}$ & & & & & $\begin{array}{l}5.7 \mathrm{E}-5 / \mathrm{h} \\
{[2]}\end{array}$ & $\begin{array}{l}6.9 \mathrm{E}-4 / \mathrm{h} \\
{[2]}\end{array}$ & $2.0 \mathrm{E}-4 / \mathrm{h}(10)$ \\
\hline $\begin{array}{l}\text { Precipitator } \\
\text { Failure }\end{array}$ & & & & & & $4.6 \mathrm{E}-5 / \mathrm{h}$ & $4.6 \mathrm{E}-5 / \mathrm{h}(10)$ \\
\hline
\end{tabular}

Table 5d. Category 3 estimates for HVAC/exhaust system.

2. Failure rate estimates are listed as: mean frequency (error factor), where the error factor is the 95 th percentile/50th percentile. Numbers in brackets refer to notes listed below, arranged by source.

b. Aggregated results are mean and error factor (in parentheses).

Notes

WASH-1400 - Reactor Safety Study: An Assessment of Accident Risks in U.S. Commercial Nuclear Power Plants, U. S. Nuclear Regulatory Commission, WASH-1400 (NUREG 75/014), October 1975.

All failure rates were obtained from Tables III 4-1 and 4-2, unless otherwise indicated. The median values listed in WASH-1400 were converted to mean values using the error factor indicated and assuming a lognormai distribution.

CCPS - Guidelines for Process Equipment Reliability Data With Data Tabies, Center for Chemical Process Safety, American Institute of Chemical Engineers, 1989.

Error factor estimates were obtained from the square root of the upper bound times the lower bound.

IEEE - IEEE Guide to the Collection end Presentaion of Electrical. Electronic. Sensine Component and Mechanical Equipment Reliability Data for Nuclear-Power Generatine Stations, Institure of Electrical and Electronic Engineers, Inc., IEEE Std 500-1984, 1983.

Recommended estimates were interpreted to be medians. Recommended estimates were converted to means using the error factor (upper bound/median) and assuming a lognormal distribution. unless otherwise indicated. If the error factor was larger than 50, it was reduced to 50 .

1. Faikure modes not indicated. 
2. No upper or lower bounds given. Recommended value interpreted as a mean because of the sources used to estimate the value.

3. Chiller data used (p. 1388).

LNG2 - H. Lammerse and M. Bosman, "Data of Gas Compressors and Instrumentation - Hard to Collect, Easy to Analyse," Reliability Engineering 13 (1985), pp. 65-78.

All estimates were taken from the observed data on p. 78.

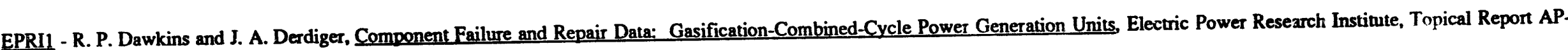
2205, Research Project 239-2, February 1982.

1. Failure modes not indicated.

2. Estimate for ammonia or particulate scrubber.

Table 5d. Category 3 estimates for HVAC/exhaust system.

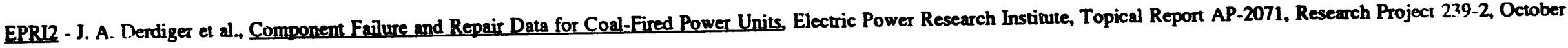
1981.

1. Failure modes not indicated.

2. Estimate for $\mathrm{SO}_{2}$ scrubber.

\section{Agregated Results}

1. Failure modes not indicated.

2. CCPS value doubled beiore combining with other estimates (assumes equal failure rates for fails to start and fails to run). 


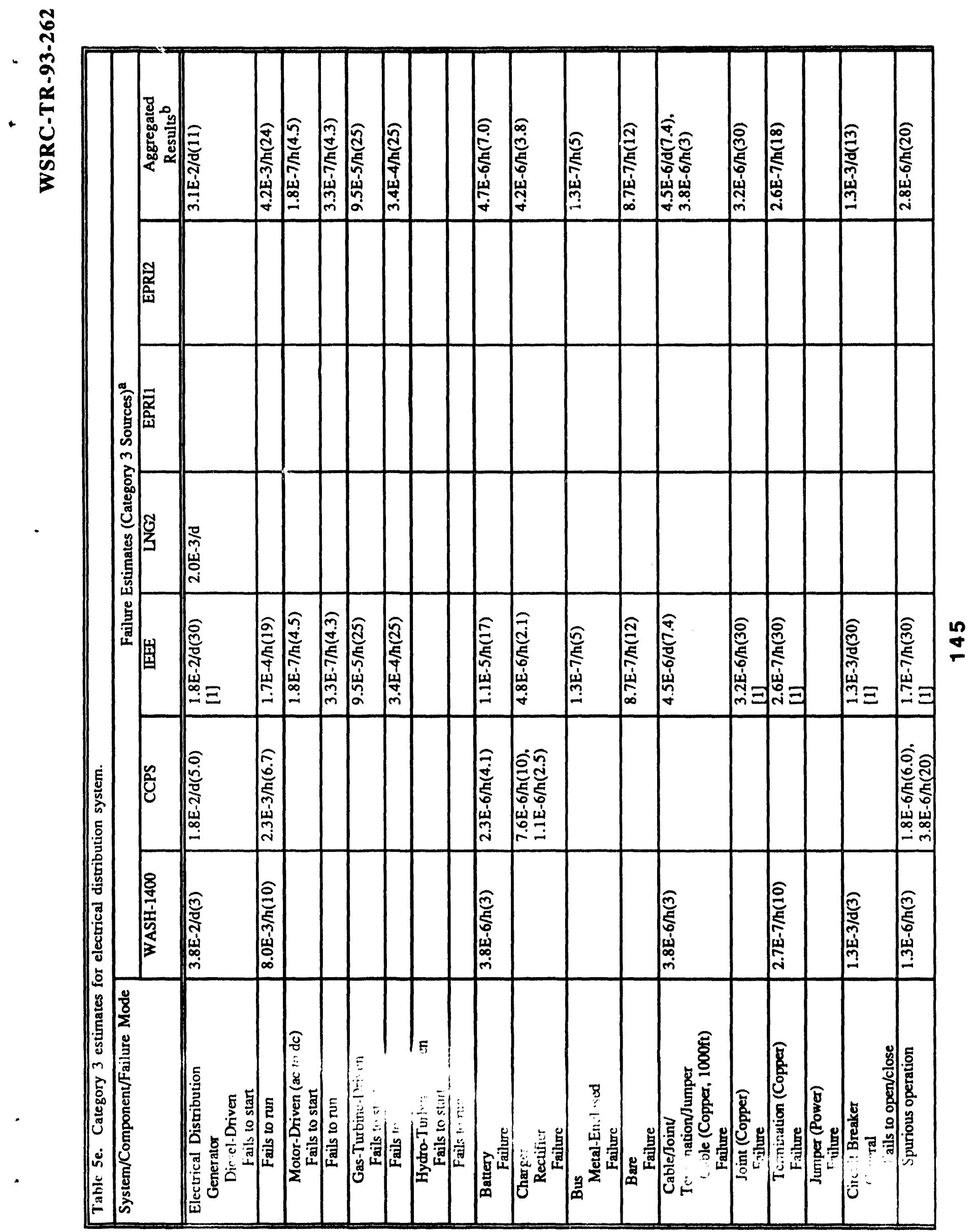


WSRC-TR-93-262

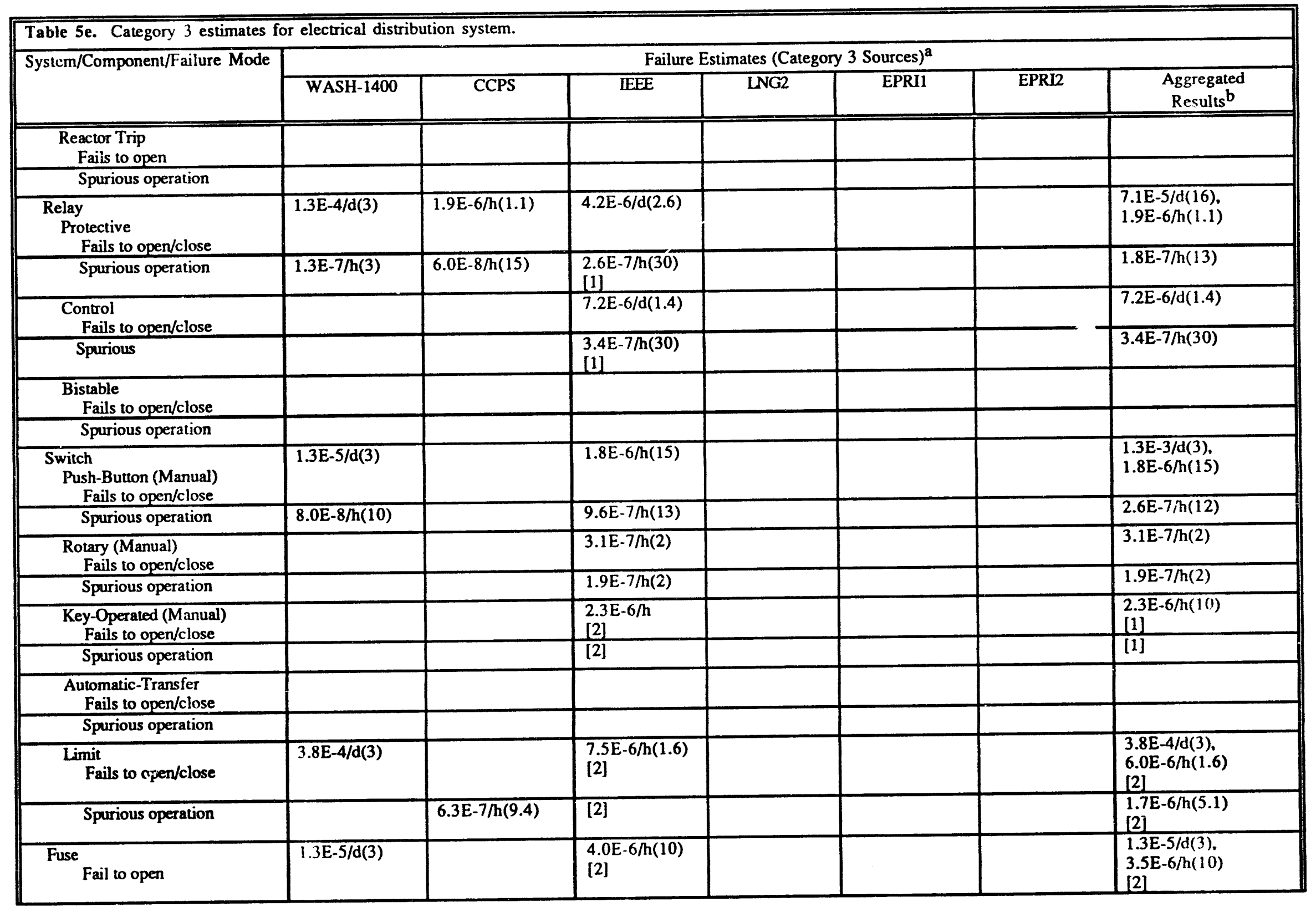

\section{6}


WSRC-TR-93-262

\begin{tabular}{|c|c|c|c|c|c|c|c|}
\hline \multirow[t]{2}{*}{ System/Component/l ailure Mode } & \multicolumn{7}{|c|}{ Failure Estimates (Category 3 Sources) ${ }^{\mathrm{a}}$} \\
\hline & WASH-1400 & CCPS & IEEE & LNG2 & EPRI1 & EPR12 & $\begin{array}{c}\text { Aggregated } \\
\text { Results } \\
\end{array}$ \\
\hline Premature opening & $1.3 \mathrm{E}-6 / \mathrm{h}(3)$ & & [2] & & & & $\begin{array}{l}1.1 \mathrm{E}-6 / \mathrm{h}(6.1) \\
{[2]}\end{array}$ \\
\hline $\begin{array}{l}\text { Inverter } \\
\text { Failure }\end{array}$ & & $2.9 \mathrm{E}-5 / \mathrm{h}(11)$ & $2.3 \mathrm{E}-5 / \mathrm{h}(13)$ & & & & $2.6 \mathrm{E}-5 / \mathrm{h}(12)$ \\
\hline $\begin{array}{l}\text { Motor } \\
\text { AC } \\
\quad \text { Fails to start }\end{array}$ & $\begin{array}{l}3.8 \mathrm{E}-4 / \mathrm{d}(3) \\
{[1]}\end{array}$ & $\begin{array}{l}1.5 \mathrm{E}-5 / \mathrm{h}(30) \\
{[1,2]}\end{array}$ & $\begin{array}{l}2.5 \mathrm{E}-5 / \mathrm{h}(30) \\
{[1]}\end{array}$ & & & & $\begin{array}{l}3.8 \mathrm{E}-4 / \mathrm{d}(3) \\
1.8 \mathrm{E}-5 / \mathrm{h}(17) \\
{[3]}\end{array}$ \\
\hline Fails to nun & $\begin{array}{l}1.3 \mathrm{E}-5 / \mathrm{h}(3) \\
{[1]}\end{array}$ & [1] & $\begin{array}{l}3.3 \mathrm{E}-5 / \mathrm{h}(30) \\
{[1]}\end{array}$ & & & & $\begin{array}{l}2.4 \mathrm{E}-5 / \mathrm{h}(17) \\
{[3]}\end{array}$ \\
\hline $\begin{array}{l}C_{\text {Fails to start }} \\
\text {. }\end{array}$ & $\begin{array}{l}3.8 \mathrm{E}-4 / \mathrm{d}(3) \\
{[1]}\end{array}$ & $\begin{array}{l}2.3 \mathrm{E}-5 / \mathrm{h}(2.5) \\
{[1]}\end{array}$ & $\begin{array}{l}1.3 \mathrm{E}-4 / \mathrm{h}(30) \\
{[1]}\end{array}$ & & & & $\begin{array}{l}3.8 \mathrm{E}-4 / \mathrm{d}(3) \\
1.8 \mathrm{E}-5 / \mathrm{h}(8.4) \\
{[3]}\end{array}$ \\
\hline Fails to nur & $\begin{array}{l}1.3 \mathrm{E}-5 / \mathrm{h}(3) \\
{[1]}\end{array}$ & [1] & [1] & & & & $\begin{array}{l}2.4 \mathrm{E}-5 / \mathrm{h}(8.4) \\
{[3]}\end{array}$ \\
\hline $\begin{array}{l}\text { Synchro } \\
\text { Failure }\end{array}$ & & & & & & & \\
\hline $\begin{array}{l}\text { Transformer } \\
\text { Power } \\
\text { Failure } \\
\end{array}$ & $1.3 \mathrm{E}-6 / \mathrm{h}(3)$ & $2.5 \mathrm{E}-6 / \mathrm{h}(8.6)$ & $7.7 \mathrm{E}-7 / \mathrm{h}(5.6)$ & & & & $1.4 \mathrm{E}-6 / \mathrm{h}(5.6)$ \\
\hline $\begin{array}{l}\text { Instrumentation Control } \\
\text { Failure }\end{array}$ & & & $3.8 \mathrm{E}-7 / \mathrm{h}(2.8)$ & & & & $3.8 \mathrm{E}-7 / \mathrm{h}(2.8)$ \\
\hline
\end{tabular}

Table Se. Category 3 estimates for electrical distribution system.

a. Failure rate estimates arc listed as: mean frequency (error factor), where the error factor is the 95th percentile/50th percentile. Numbers in brackets refer to notes listed below, arranged by source.

b. Aggregated results are mcan and error factor (in parentheses).

\section{Notes}

WASH-1400 - Reactor Safety Study: An Assessment of Accident Risks in U. S. Commercial Nuclear Power Plants, U. S. Nuciear Regulatory Commission, WASH-1400 (NUREG 75/014), October 1975.

All failure rates were obtained from Tables III 4-1 and 4-2, unless otherwise indicated. The median values listed in WASH-1400 were converted to mean values using the error factor indicated and assuming a lognormal tiscribution. 
1. Type of motor not indicated.

CCPS - Guidelines for Process Equipment Reliability Data With Data Tables, Center for Chemical Process Safety, American Institute of Chemical Engineers, 1989.

Enror factor estimates were obtained from the square root of the upper bound times the lower bound.

1. Failure modes not indicated.

2. Error factor reduced to 30 .

IEEE - IEEE Guide to the Collection and Presentation of Electrical, Electronic, Sensing Component, and Mechanical Equipment Reliability Data for Nuclear-Power Generating Stations, Institute of Electrical and Electronic Engineers, Inc., IEEE Std 500-1984, 1983.

Recommended est imates were interpreted to be medians. Recommended estimates were converted to means using the error factor (upper bound/median) and assuming a lognormal distribution, unless otherwise inkicated. If the error factor was larger than 50 , it was reduced to 50 .

1. Error factor reduced to 30.

2. Failure mindes not indicated.

LNG2 - H. Lammrise and M. Bosman, "Data of Gas Compressors and Instrumentation - Hard to Collect, Easy to Analyse," Reliability Engineering 13 (1985), pp. 65-78.

All estimates wer: aken from the observed data on p. 78.

EPRIL - R. P. Dawkins and J. A. Derdiger, Component Failure and Repair Data: Gasification-Combined-Cycle Power Generation Units, Electric Power Research Instiute, Topical Report AP2205, Research P'oject 239-2, February 1982.

Table 5e. Categury 3 estimates for electrical distribution system.

EPRI2 - J. A. Derdiger et al., Component Failure and Repair Data for Coal-Fired Power Units, Electric Power Research Institute, Topical Report AP-2071, Research Project 239-2, October 1981.

\section{Areregated Resulis}

1. Failure modes not indicated.

2. Relay and switch results indicate approximately one-fifth of failures are spurious operation. This information was used to partition the IEEE estimate.

3. Category $\mathbf{2}$ data indicate $\mathbf{1}$ of $\mathbf{8 . 5}$ failures is premature opening. This information was used to partition the IEEE estimate. 
WSRC-TR-93-262

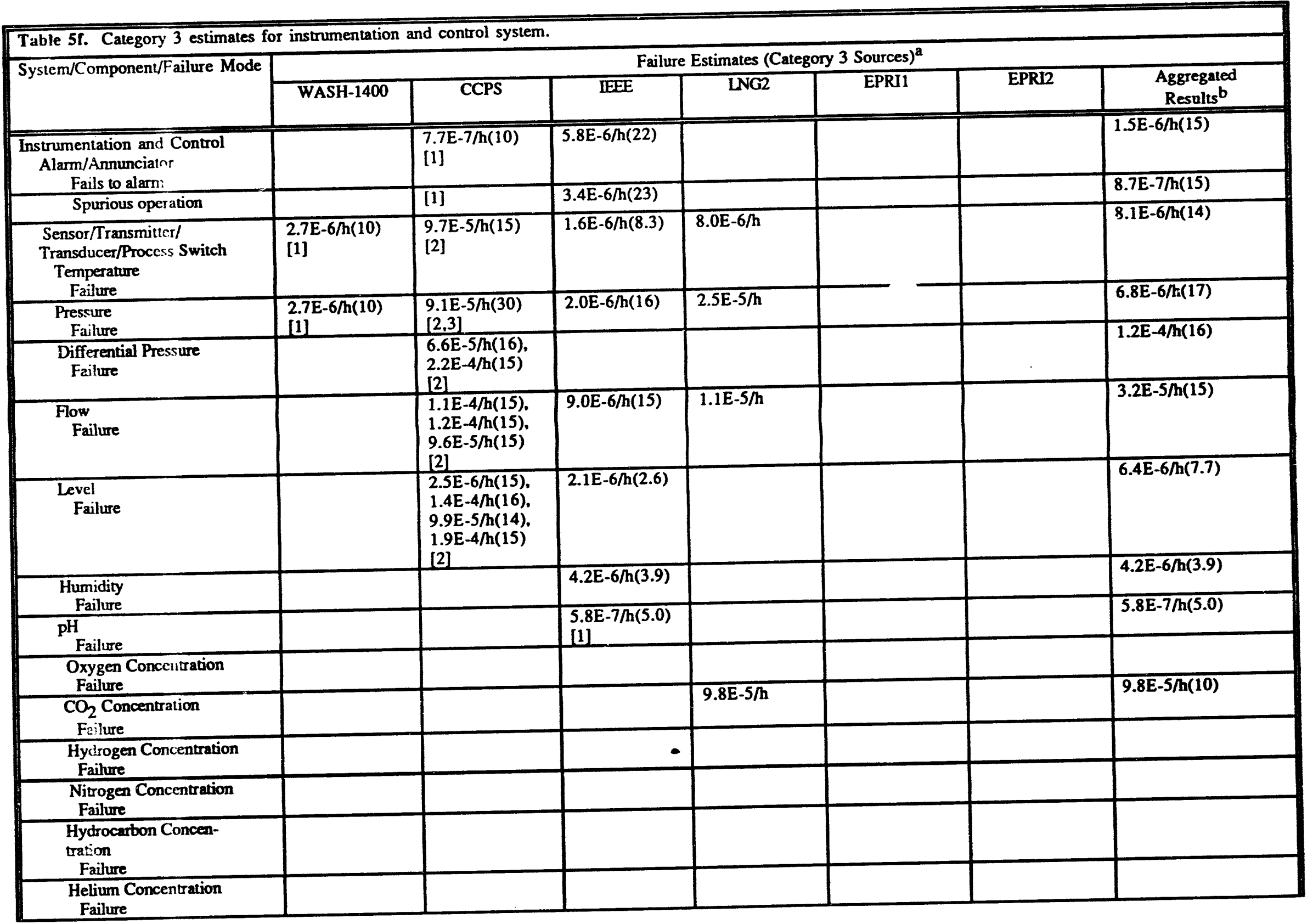




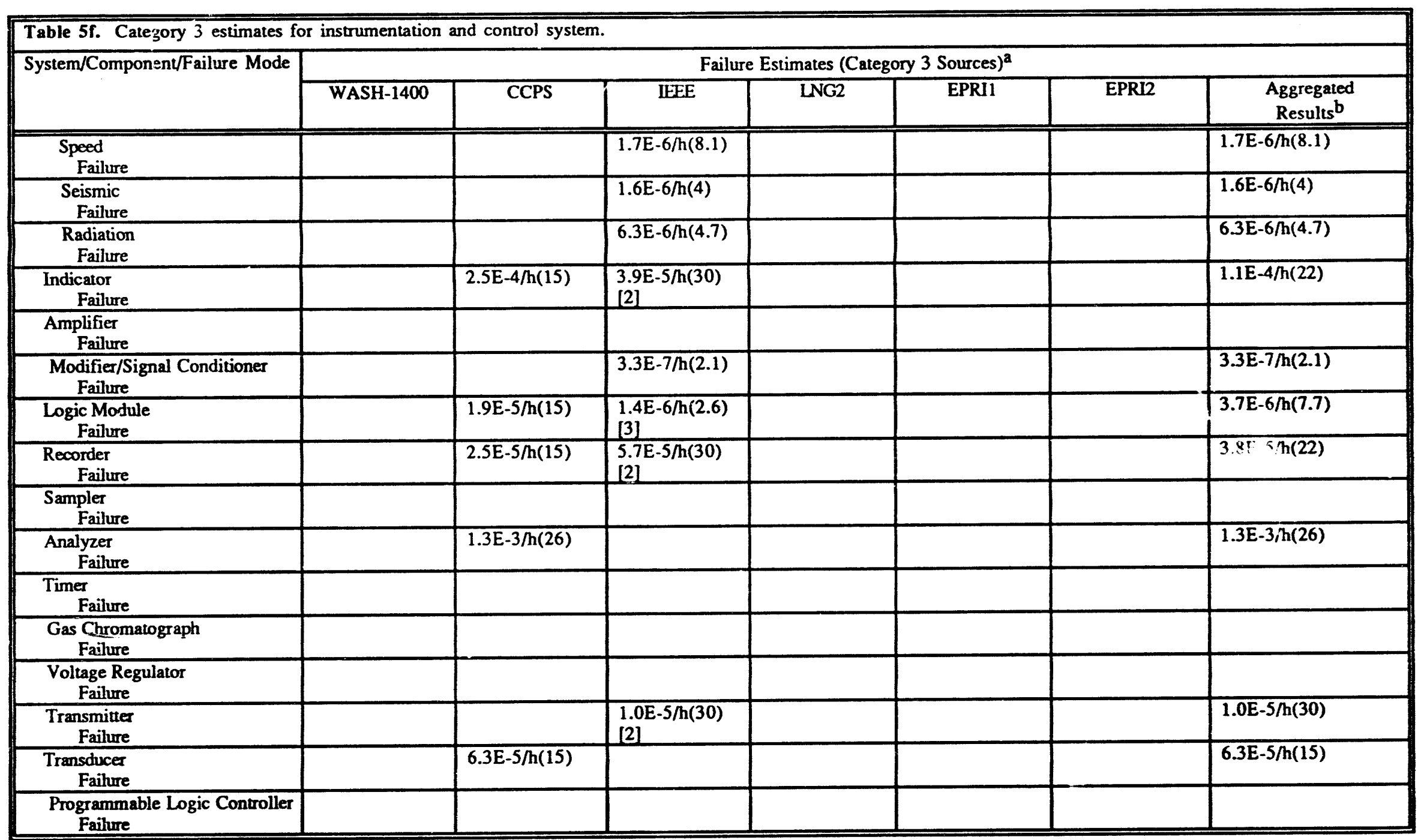

Table 5f. Category 3 estimates for instrumentation and control system.

a. Failure rate estimates are listed as: mean frequency (error factor), where the error factor is the 95th percentile/50th percentile. Numbers in brackets refer to notes listed below, arranged by source. 
$+$

b. Aggregated results are mean and error factor (in parentheses).

Notes

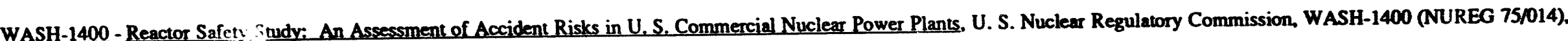
October 1975.

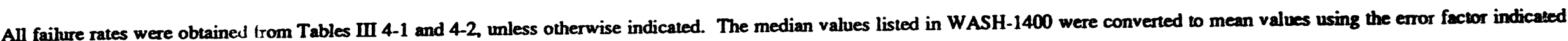
and assuming a lognormal distribution.

1. Type of instrument not indicated.

CCPS - Guidelines for Proces: Equipment Reliability Data With Data Tables, Center for Chemical Process Safety, American Instinute of Chemical Engineers, 1989.

Error factor estimates were obteined from the square root of the upper bound times the lower bound.

1. Faihure modes not indicated.

2. Transmitter estimate used.

3. Error factor reduced to 30.

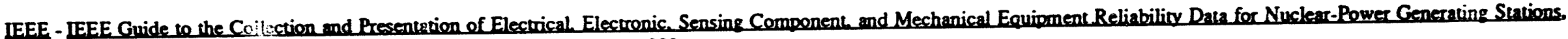
Institute of Electrical and Electronic Engineers. Inc.. IEEE Std 500-1984, 1983.

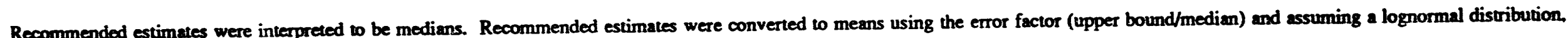
unless otherwise indicated. If the error factor was larger than 50, it was reduced to 50.

1. Water chemistry instrumentation estimate used.

2. Error factor reduced $\omega 30$.

3. Totalizer estimate used.

LNG2 - H. Lammerse and M. Bosman, "Data of Gas Compressors and Instrumentation - Hard to Collect, Easy to Analyse," Reliability Eneineering 13 (1985), pp. 65-78.

All estimates were taken from the observed data on p. 78.

Table 5f. Category 3 estimates for instrumentation and control system.

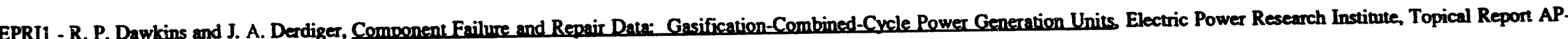
2205, Research Project 239-2, February 1982. 
WSRC-TR-93-262

EPRI2 - J. A. Derdiger et al., Component Failure and Repair Data for Coal-Fired Power Units, Electric Power Research Institute, Topical Report AP-2071, Research Project 239-2, October 1981. 
WSRC-TR-93-262

\begin{tabular}{|c|c|c|c|c|}
\hline \multirow[t]{2}{*}{ System/Component/Failure Mode } & \multicolumn{4}{|c|}{ Aggregated and Recommended Failure Rates ${ }^{\mathrm{a}}$} \\
\hline & Category 1 & Category 2 & Category 3 & Recommended $^{b}$ \\
\hline $\begin{array}{l}\text { Water } \\
\text { Valve (Standby or Safety) } \\
\text { Manual } \\
\text { Fails to open/close }\end{array}$ & $\begin{array}{l}3.5 \mathrm{E}-4 / \mathrm{d}(18) \\
3.3 \mathrm{E}-8 / \mathrm{h}(30)\end{array}$ & $\begin{array}{l}1.4 \mathrm{E}-4 / \mathrm{d}(19) \\
7.1 \mathrm{E}-7 \mathrm{~h}(10)\end{array}$ & $6.0 \mathrm{E}-5 / \mathrm{d}(10)$ & $3.0 \mathrm{E}-4 / \mathrm{d}(10)$ \\
\hline Plugs & $\begin{array}{l}1.1 \mathrm{E}-6 / \mathrm{h}(10) \\
{[1]}\end{array}$ & & $3.8 \mathrm{E}-7 / \mathrm{h}(3)$ & $\begin{array}{l}5.0 \mathrm{E}-8 / \mathrm{h}(10) \\
{[1]}\end{array}$ \\
\hline Leakage (internal) & $\begin{array}{l}5.7 \mathrm{E}-3 / \mathrm{h}(10) \\
{[1]}\end{array}$ & & & $\begin{array}{l}1.0 \mathrm{E}-6 / \mathrm{h}(10) \\
{[2]}\end{array}$ \\
\hline Rupture (internal) & & & & $\begin{array}{l}5.0 \mathrm{E}-8 / \mathrm{h}(30) \\
{[2]}\end{array}$ \\
\hline Leakage (external) & & $1.0 \mathrm{E}-8 / \mathrm{h}(9.6)$ & $2.0 \mathrm{E}-8 / \mathrm{h}(10)$ & $\begin{array}{l}1.0 \mathrm{E}-8 / \mathrm{h}(10) \\
{[3]}\end{array}$ \\
\hline Rupture (external) & & $5.8 \mathrm{E}-10 / \mathrm{h}(10)$ & $2.7 \mathrm{E}-8 / \mathrm{h}(10)$ & $\begin{array}{l}5.0 \mathrm{E}-10 \mathrm{~h}(30) \\
{[3]}\end{array}$ \\
\hline $\begin{array}{l}\text { Check } \\
\text { Fails to open }\end{array}$ & $6.0 \mathrm{E}-5 / \mathrm{d}(10)$ & $\begin{array}{l}1.8 \mathrm{E}-5 / \mathrm{d}(10) \\
1.3 \mathrm{E}-7 / \mathrm{h}(10)\end{array}$ & $8.8 \mathrm{E}-5 / \mathrm{d}(3.0)$ & $5.0 \mathrm{E}-5 / \mathrm{d}(10)$ \\
\hline Fails to close & $9.8 \mathrm{E}-4 / \mathrm{d}(4.6)$ & $\begin{array}{l}\text { 5.7E-5/d(10), } \\
3.9 \mathrm{E}-7 / \mathrm{h}(10)\end{array}$ & & $1.0 \mathrm{E}-3 / \mathrm{d}(5)$ \\
\hline Plugs & & & & $\begin{array}{l}5.0 \mathrm{E}-8 / \mathrm{h}(10) \\
{[1]}\end{array}$ \\
\hline Leakage (intermal) & $6.8 \mathrm{E}-7 / \mathrm{h}(30)$ & $9.1 \mathrm{E}-7 / \mathrm{h}(2.9)$ & $5.0 \mathrm{E}-7 / \mathrm{h}(5.4)$ & $\begin{array}{l}1.0 \mathrm{E}-6 / \mathrm{h}(10) \\
{[2]}\end{array}$ \\
\hline Rupture (internal) & & & $1.9 \mathrm{E}-7 / \mathrm{h}(5.4)$ & $5.0 \mathrm{E}-8 / \mathrm{h}(30)$ \\
\hline Leakage (extemal) & & $1.5 \mathrm{E}-8 / \mathrm{h}(26)$ & & $\begin{array}{l}1.0 \mathrm{E}-8 / \mathrm{h}(10) \\
{[3]}\end{array}$ \\
\hline Rupture (external) & & $5.8 \mathrm{E}-10 / \mathrm{h}(10)$ & $2.7 \mathrm{E}-8 / \mathrm{h}(10)$ & $\begin{array}{l}5.0 \mathrm{E}-10 \mathrm{~h}(30) \\
{[3]}\end{array}$ \\
\hline $\begin{array}{l}\text { Motor-Operated } \\
\text { Fails to men/close }\end{array}$ & $\begin{array}{l}3.3 \mathrm{E}-3 / \mathrm{d}(6.3) \\
4.8 \mathrm{E}-6 / \mathrm{h}(9.6)\end{array}$ & $3.8 \mathrm{E}-3 / \mathrm{d}(1.8)$ & $3.0 \mathrm{E}-3 / \mathrm{d}(3.5)$ & $3.0 \mathrm{E}-3 / \mathrm{d}(5)$ \\
\hline Spuris: reration & $2.3 \mathrm{E}-7 / \mathrm{h}(7.2)$ & $1.8 \mathrm{E}-7 / \mathrm{h}(11)$ & & $3.0 \mathrm{E}-7 / \mathrm{h}(5)$ \\
\hline Plugs & $5.6 \mathrm{E}-8 / \mathrm{h}(10)$ & & $1.9 \mathrm{E}-7 / \mathrm{h}(8.1)$ & $\begin{array}{l}5.0 \mathrm{E}-8 / \mathrm{h}(10) \\
{[1]}\end{array}$ \\
\hline Leakage (intemal) & $8.3 \mathrm{E}-7 \mathrm{~h}(10)$ & & & $\begin{array}{l}1.0 \mathrm{E}-6 / \mathrm{h}(10) \\
{[2]}\end{array}$ \\
\hline Rupture (internal) & & & & $\begin{array}{l}5.0 \mathrm{E}-8 / \mathrm{h}(30) \\
{[2]}\end{array}$ \\
\hline
\end{tabular}

153 


\begin{tabular}{|c|c|c|c|c|}
\hline \multirow[t]{2}{*}{ System/Component/Failure Mode } & \multicolumn{4}{|c|}{ Aggregated and Recommended Failure Rates ${ }^{\mathbf{a}}$} \\
\hline & Category 1 & Category 2 & Category 3 & Recommended $\mathrm{b}$ \\
\hline Leakage (extemal) & & $9.6 \mathrm{E}-9 / \mathrm{h}(10)$ & $1.0 \mathrm{E}-7 / \mathrm{h}(10)$ & $\begin{array}{l}1.0 \mathrm{E}-8 / \mathrm{h}(10) \\
{[3]}\end{array}$ \\
\hline Rupture (extemal) & & $5.8 \mathrm{E}-10 / \mathrm{h}(10)$ & $2.7 \mathrm{E}-8 / \mathrm{h}(10)$ & $\begin{array}{l}5.0 \mathrm{E}-10 / \mathrm{h}(30) \\
{[3]}\end{array}$ \\
\hline $\begin{array}{l}\text { Air-Operated } \\
\text { Fails to open/close }\end{array}$ & $\begin{array}{l}1.2 \mathrm{E}-3 / \mathrm{d}(21), \\
5.2 \mathrm{E}-7 / \mathrm{h}(10)\end{array}$ & $3.4 \mathrm{E}-3 / \mathrm{d}(3.8)$ & $9.8 \mathrm{E}-4 / \mathrm{d}(3.9)$ & $1.0 \mathrm{E}-3 / \mathrm{d}(30)$ \\
\hline Spurious operation & $1.2 \mathrm{E}-6 / \mathrm{h}(5.4)$ & $1.2 \mathrm{E}-6 / \mathrm{h}(2.0)$ & & $1.0 \mathrm{E}-6 / \mathrm{h}(5)$ \\
\hline Plugs & $\begin{array}{l}1.1 \mathrm{E}-7 / \mathrm{h}(10) \\
{[1]}\end{array}$ & & $3.8 \mathrm{E}-7 / \mathrm{h}(3)$ & $\begin{array}{l}5.0 \mathrm{E}-8 / \mathrm{h}(10) \\
{[1]}\end{array}$ \\
\hline Leakagc (intemal) & $1.6 \mathrm{E}-6 / \mathrm{h}(10)$ & $6.9 \mathrm{E}-7 / \mathrm{h}(2.6)$ & & $\begin{array}{l}1.0 \mathrm{E}-6 / \mathrm{h}(10) \\
{[2]}\end{array}$ \\
\hline Rupture (internal) & & $3.0 \mathrm{E}-7 / \mathrm{h}(2.6)$ & & $\begin{array}{l}5.0 \mathrm{E}-8 / \mathrm{h}(30) \\
{[2]}\end{array}$ \\
\hline Leakage (external) & & $9.9 \mathrm{E}-9 / \mathrm{h}(7.6)$ & $1.0 \mathrm{E}-7 / \mathrm{h}(10)$ & $\begin{array}{l}1.0 \mathrm{E}-8 / \mathrm{h}(10) \\
{[3]}\end{array}$ \\
\hline Rupture (external) & & $5.8 \mathrm{E}-10 / \mathrm{h}(10)$ & $2.7 \mathrm{E}-8 / \mathrm{h}(10)$ & $\begin{array}{l}5.0 \mathrm{E}-10 / \mathrm{h}(30) \\
{[3]}\end{array}$ \\
\hline $\begin{array}{l}\text { Solenoid-Operated } \\
\text { Fails to open/close }\end{array}$ & $\begin{array}{l}7.8 \mathrm{E}-4 / \mathrm{d}(10), \\
7.1 \mathrm{E}-7 / \mathrm{h}(10)\end{array}$ & $\begin{array}{l}1.1 \mathrm{E}-2 / \mathrm{d}(10) \\
7.5 \mathrm{E}-8 \mathrm{~h}(10)\end{array}$ & $1.3 \mathrm{E}-3 / \mathrm{d}(3)$ & $1.0 \mathrm{E}-3 / \mathrm{d}(10)$ \\
\hline Spurious operation & $6.1 \mathrm{E}-7 / \mathrm{h}(10)$ & $5.6 \mathrm{E}-8 / \mathrm{h}(10)$ & & $5.0 \mathrm{E}-7 / \mathrm{h}(10)$ \\
\hline Plugs & $3.3 \mathrm{E}-4 / \mathrm{h}(10)$ & & $3.8 \mathrm{E}-7 / \mathrm{h}(3)$ & $\begin{array}{l}5.0 \mathrm{E}-8 / \mathrm{h}(10) \\
{[1]}\end{array}$ \\
\hline Leakage (internal) & & & & $\begin{array}{l}1.0 \mathrm{E}-6 / \mathrm{h}(10) \\
{[2]}\end{array}$ \\
\hline Rupture (internal) & & & & $\begin{array}{l}5.0 \mathrm{E}-8 / \mathrm{h}(30) \\
{[2]}\end{array}$ \\
\hline Leakage (extemal) & & $1.3 \mathrm{E}-8 / \mathrm{h}(30)$ & & [3] \\
\hline Rupture (external) & & $5.8 \mathrm{E}-10 / \mathrm{h}(10)$ & $2.7 \mathrm{E}-8 / \mathrm{h}(10)$ & $\begin{array}{l}5.0 \mathrm{E}-10 / \mathrm{h}(30) \\
{[3]}\end{array}$ \\
\hline $\begin{array}{l}\text { Safety/Relief } \\
\text { Fails to open }\end{array}$ & $\begin{array}{l}2.7 \mathrm{E}-3 / \mathrm{d}(3.3), \\
8.5 \mathrm{E}-8 / \mathrm{h}(10) \\
{[1]}\end{array}$ & $1.2 \mathrm{E}-2 / \mathrm{d}(3.3)$ & $1.5 \mathrm{E}-3 / \mathrm{d}(30)$ & $3.0 \mathrm{E}-3 / \mathrm{d}(3)$ \\
\hline Fails to reclose & $2.3 \mathrm{E}-3 / \mathrm{d}(1.7)$ & & & $3.0 \mathrm{E}-3 / \mathrm{d}(3)$ \\
\hline
\end{tabular}


Table 6a. Summary of results for water system.

\begin{tabular}{|c|c|c|c|c|}
\hline \multirow[t]{2}{*}{ System,'Component/Failure Mode } & \multicolumn{4}{|c|}{ Aggregated and Recommended Failure Rates ${ }^{a}$} \\
\hline & Category 1 & Category 2 & Category 3 & Recommended ${ }^{b}$ \\
\hline Leakage (intemal) & & $1.1 \mathrm{E}-6 / \mathrm{h}(2.5)$ & $1.5 \mathrm{E}-5 / \mathrm{h}(3)$ & $\begin{array}{l}1.0 \mathrm{E}-6 / \mathrm{h}(10) \\
{[2]}\end{array}$ \\
\hline Rupwure (intemal) & & & $3.0 \mathrm{E}-6 / \mathrm{h}(10)$ & $\begin{array}{l}5.0 \mathrm{E}-8 / \mathrm{h}(30) \\
{[2]}\end{array}$ \\
\hline Leakage (extemal) & & $1.1 \mathrm{E}-6 / \mathrm{h}(2.5)$ & & $\begin{array}{l}1.0 \mathrm{E}-8 / \mathrm{h}(10) \\
{[3]}\end{array}$ \\
\hline Rupture (external) & & $4.9 \mathrm{E}-8 / \mathrm{h}(10)$ & & $\begin{array}{l}5.0 \mathrm{E}-10 / \mathrm{h}(30) \\
{[3]}\end{array}$ \\
\hline $\begin{array}{l}\text { Vacuum-Breaker } \\
\text { Fails to open }\end{array}$ & $\begin{array}{l}1.1 \mathrm{E}-2 / \mathrm{d}(10) \\
4.4 \mathrm{E}-4 / \mathrm{h}(10) \\
{[1]}\end{array}$ & & $3.8 \mathrm{E}-5 / \mathrm{d}(3)$ & $1.0 \mathrm{E}-2 / \mathrm{d}(10)$ \\
\hline Fails to reclose & & & & $\begin{array}{l}1.0 \mathrm{E}-2 / \mathrm{d}(10) \\
{[4]}\end{array}$ \\
\hline Leakaze (intemal) & & & & $\begin{array}{l}1.0 \mathrm{E}-6 / \mathrm{h}(10) \\
{[2]}\end{array}$ \\
\hline Rupture (internal) & & & & $\begin{array}{l}5.0 \mathrm{E}-8 / \mathrm{h}(30) \\
{[2]}\end{array}$ \\
\hline Leakage (external) & & & & $\begin{array}{l}1.0 \mathrm{E}-8 / \mathrm{h}(10) \\
{[3]}\end{array}$ \\
\hline Rupture (external) & & & & $\begin{array}{l}5.0 \mathrm{E}-10 / \mathrm{h}(30) \\
{[3]}\end{array}$ \\
\hline $\begin{array}{l}\text { Explosive } \\
\text { Fails to open }\end{array}$ & $\begin{array}{l}1.8 \mathrm{E}-2 / \mathrm{d}(10) \\
{[1]}\end{array}$ & & & $\begin{array}{l}1.0 \mathrm{E}-4 / \mathrm{d}(10) \\
{[5]}\end{array}$ \\
\hline Leakage (internal) & & & & $\begin{array}{l}1.0 \mathrm{E}-6 / \mathrm{h}(10) \\
{[2]}\end{array}$ \\
\hline Rupture (intemal) & & & & $\begin{array}{l}5.0 \mathrm{E}-8 / \mathrm{h}(30) \\
{[2]}\end{array}$ \\
\hline Leakage (extemal) & & & & $\begin{array}{l}1.0 \mathrm{E}-8 / \mathrm{h}(10) \\
{[3]}\end{array}$ \\
\hline Rupture (extermal) & & & & $\begin{array}{l}5.0 \mathrm{E}-10 / \mathrm{h}(30) \\
{[3]}\end{array}$ \\
\hline $\begin{array}{l}\text { Valve (Control) } \\
\text { Motor-Operated } \\
\text { Fails open }\end{array}$ & & & & $\begin{array}{l}3.0 \mathrm{E}-6 / \mathrm{h}(10) \\
{[6]}\end{array}$ \\
\hline Fails closed & & & & $\begin{array}{l}3.0 \mathrm{E}-6 / \mathrm{h}(10) \\
{[6]}\end{array}$ \\
\hline
\end{tabular}


Table 6a. Summary of results for water system.

\begin{tabular}{|c|c|c|c|c|}
\hline \multirow{2}{*}{ System/Component/Failure Mode } & \multicolumn{4}{|c|}{ Aggregated and Recommended Failure Rates ${ }^{a}$} \\
\hline & Category 1 & Category 2 & Category 3 & Recommended $\mathbf{b}$ \\
\hline Fails to respond & & & & $\begin{array}{l}3.0 \mathrm{E}-6 / \mathrm{h}(10) \\
{[6]}\end{array}$ \\
\hline Plugs & & & & $\begin{array}{l}5.0 \mathrm{E}-8 / \mathrm{h}(10) \\
{[1]}\end{array}$ \\
\hline Leakage (extemal) & & & & $\begin{array}{l}1.0 \mathrm{E}-8 / \mathrm{h}(10) \\
{[3]}\end{array}$ \\
\hline Rupture (external) & & & & $\begin{array}{l}5.0 \mathrm{E}-10 / \mathrm{h}(30) \\
{[3]}\end{array}$ \\
\hline $\begin{array}{c}\text { Air-Operated } \\
\text { Fails open }\end{array}$ & & & & $\begin{array}{l}3.0 \mathrm{E}-6 / \mathrm{h}(10) \\
{[6]}\end{array}$ \\
\hline Fails closed & & & & $\begin{array}{l}3.0 \mathrm{E}-6 / \mathrm{h}(10) \\
{[6]}\end{array}$ \\
\hline Fails to respond & & & & $\begin{array}{l}3.0 \mathrm{E}-6 / \mathrm{h}(10) \\
{[6]}\end{array}$ \\
\hline Plugs & & & & $\begin{array}{l}5.0 \mathrm{E}-8 / \mathrm{h}(10) \\
{[1]}\end{array}$ \\
\hline Leakage (external) & & & & $\begin{array}{l}1.0 \mathrm{E}-8 / \mathrm{h}(10) \\
{[3]}\end{array}$ \\
\hline Rupure (external) & & & & $\begin{array}{l}5.0 \mathrm{E}-10 \mathrm{~h}(30) \\
{[3]}\end{array}$ \\
\hline $\begin{array}{l}\text { Solenoid-Operated } \\
\text { Fails open }\end{array}$ & & & & $\begin{array}{l}3.0 \mathrm{E}-6 / \mathrm{h}(10) \\
{[6]}\end{array}$ \\
\hline Fails closed & & & & $\begin{array}{l}3.0 \mathrm{E}-6 / \mathrm{h}(10) \\
{[6]}\end{array}$ \\
\hline Fails to respond & & & & $\begin{array}{l}3.0 \mathrm{E}-6 / \mathrm{h}(10) \\
{[6]}\end{array}$ \\
\hline Phugs & & & & $\begin{array}{l}5.0 \mathrm{E}-8 / \mathrm{h}(10) \\
{[1]}\end{array}$ \\
\hline Leakage (extemal) & & & & $\begin{array}{l}1.0 \mathrm{E}-8 / \mathrm{h}(10) \\
{[3]}\end{array}$ \\
\hline Ruphure (external) & & & & $\begin{array}{l}5.0 \mathrm{E}-10 / \mathrm{h}(30) \\
{[3]}\end{array}$ \\
\hline $\begin{array}{l}\text { Purmp } \\
\text { Motor-Driven } \\
\text { Fails to start }\end{array}$ & 3.7E-3/d(4.7) & $\begin{array}{l}8.3 \mathrm{E}-4 / \mathrm{d}(9.1) \\
3.7 \mathrm{E}-6 / \mathrm{h}(8.3)\end{array}$ & $\begin{array}{l}7.5 \mathrm{E}-3 / \mathrm{d}(8.3) \\
2.4 \mathrm{E}-5 / \mathrm{h}(5.8)\end{array}$ & $3.0 \mathrm{E}-3 / \mathrm{d}(5)$ \\
\hline Fails to num & $2.2 \mathrm{E}-5 / \mathrm{h}(17)$ & $9.4 \mathrm{E}-6 / \mathrm{h}(11)$ & $2.1 \mathrm{E}-5 / \mathrm{h}(6.8)$ & $3.0 \mathrm{E}-5 / \mathrm{h}(10)$ \\
\hline Overspeed & & $1.9 \mathrm{E}-6 / \mathrm{h}(11)$ & & $5.0 \mathrm{E}-6 / \mathrm{h}(10)$ \\
\hline
\end{tabular}


WSRC-TR-93-262

\begin{tabular}{|c|c|c|c|c|}
\hline \multirow[t]{2}{*}{ System/Component/Failure Mode } & \multicolumn{4}{|c|}{ Aggregated and Recommended Failure Rates ${ }^{a}$} \\
\hline & Category 1 & Category 2 & Category 3 & Recommendedb \\
\hline Fails to stop & $3.8 \mathrm{E}-3 / \mathrm{d}(4.4)$ & & & $3.0 \mathrm{E}-3 / \mathrm{d}(5)$ \\
\hline Leakage (extemal) & & $5.9 \mathrm{E}-8 / \mathrm{h}(30)$ & & $\begin{array}{l}3.0 \mathrm{E}-8 / \mathrm{h}(10) \\
{[3]}\end{array}$ \\
\hline Rupture (external) & & $1.7 \mathrm{E}-9 / \mathrm{h}(10)$ & & $\begin{array}{l}1.0 \mathrm{E}-9 / \mathrm{h}(30) \\
{[3]}\end{array}$ \\
\hline $\begin{array}{l}\text { Turbine-Driven } \\
\text { Fails to start }\end{array}$ & $\begin{array}{l}2.2 \mathrm{E}-2 / \mathrm{d}(3.8) \\
5.5 \mathrm{E}-5 / \mathrm{h}(10)\end{array}$ & $1.0 \mathrm{E}-2 / \mathrm{d}(10)$ & $\begin{array}{l}\text { 4.0E-3/d(10), } \\
3.8 \mathrm{E}-5 / \mathrm{h}(10)\end{array}$ & $3.0 \mathrm{E}-2 / \mathrm{d}(3)$ \\
\hline Fails to run & $1.4 \mathrm{E}-4 / \mathrm{h}(22)$ & $1.0 \mathrm{E}-4 / \mathrm{h}(1.7)$ & $3.8 \mathrm{E}-5 / \mathrm{h}(10)$ & $1.0 \mathrm{E}-4 / \mathrm{h}(30)$ \\
\hline Overspeed & & & & $\begin{array}{l}3.0 \mathrm{E}-5 / \mathrm{h}(10) \\
{[7]}\end{array}$ \\
\hline Fails to stop & & & & $\begin{array}{l}3.0 \mathrm{E}-2 / \mathrm{d}(3) \\
{[7]}\end{array}$ \\
\hline Leakage (external) & & $2.7 \mathrm{E}-8 / \mathrm{h}(10)$ & & $\begin{array}{l}3.0 \mathrm{E}-8 / \mathrm{h}(10) \\
{[3]}\end{array}$ \\
\hline Rupture (external) & & $1.6 \mathrm{E}-9 / \mathrm{h}(10)$ & & $\begin{array}{l}1.0 \mathrm{E}-9 / \mathrm{h}(30) \\
{[3]}\end{array}$ \\
\hline $\begin{array}{l}\text { Diesel-Driven } \\
\text { Fails to start }\end{array}$ & $7.9 \mathrm{E}-3 / \mathrm{d}(5.8)$ & $3.4 \mathrm{E}-2 / \mathrm{d}(4.0)$ & $9.8 \mathrm{E}-3 / \mathrm{d}(9.8)$ & $1.0 \mathrm{E}-2 / \mathrm{d}(5)$ \\
\hline Fails to nun & $4.6 \mathrm{E}-3 / \mathrm{h}(7.4)$ & $2.3 \mathrm{E}-2 / \mathrm{h}(10)$ & & $5.0 \mathrm{E}-3 / \mathrm{h}(5)$ \\
\hline Overspeed & & & & $\begin{array}{l}1.0 \mathrm{E}-3 / \mathrm{h}(5) \\
{[7]}\end{array}$ \\
\hline Fails to stop & & & & $1.0 \mathrm{E}-2 / \mathrm{d}(5)$ \\
\hline Leakage (external) & & $2.7 \mathrm{E}-8 / \mathrm{h}(10)$ & & $\begin{array}{l}3.0 \mathrm{E}-8 / \mathrm{h}(10) \\
{[3]}\end{array}$ \\
\hline Rupture (external) & & $1.6 \mathrm{E}-9 / \mathrm{h}(10)$ & & $\begin{array}{l}1.0 \mathrm{E}-9 / \mathrm{h}(30) \\
{[3]}\end{array}$ \\
\hline $\begin{array}{l}\text { Piping/Hose/Jumper } \\
\text { Piping } \\
\text { Leakage (external) }\end{array}$ & & $2.3 \mathrm{E}-9 / \mathrm{h}-\mathrm{ft}(10)$ & 2.7E-9/h-ft(30) & $\begin{array}{l}3.0 \mathrm{E}-9 / \mathrm{h}-\mathrm{ft}(10) \\
{[3]}\end{array}$ \\
\hline Rupture (extemal) & & $5.8 \mathrm{E}-11 / \mathrm{h}-\mathrm{ft}(10)$ & $1.3 \mathrm{E}-10 / \mathrm{h}-\mathrm{fr}(30)$ & $\begin{array}{l}1.0 \mathrm{E}-10 / \mathrm{h}-\mathrm{ft}(30) \\
\text { [3] }\end{array}$ \\
\hline Plugs & & & $1.3 \mathrm{E}-10 / \mathrm{h}-\mathrm{ft}(30)$ & $\begin{array}{l}1.0 \mathrm{E}-10 / \mathrm{h}-\mathrm{ft}(30) \\
{[8]}\end{array}$ \\
\hline $\begin{array}{l}\text { Hose } \\
\text { Leakage (external) }\end{array}$ & & $1.7 E-9 / h-f t(10)$ & & $1.0 \mathrm{E}-9 / \mathrm{h}-\mathrm{ft}(10)$ \\
\hline
\end{tabular}




\begin{tabular}{|c|c|c|c|c|}
\hline \multirow[t]{2}{*}{ System/Component/Failure Mode } & \multicolumn{4}{|c|}{ Aggregated and Recommended Failure Rates } \\
\hline & Category 1 & Category 2 & Category 3 & Recommended $b$ \\
\hline Rupture (external) & & $1.0 \mathrm{E}-8 / \mathrm{h}-\mathrm{ft}(10)$ & & $1.0 \mathrm{E}-8 / \mathrm{h}-\mathrm{ft}(10)$ \\
\hline Plugs & & & & $\begin{array}{l}1.0 \mathrm{E}-8 / \mathrm{h}-\mathrm{ft}(10) \\
{[8]}\end{array}$ \\
\hline $\begin{array}{l}\text { Jumper } \\
\text { Leakage (external) }\end{array}$ & & & & $\begin{array}{l}1.0 \mathrm{E}-6 / \mathrm{h}(10) \\
{[9]}\end{array}$ \\
\hline Rupture (external) & & & & $\begin{array}{l}1.0 \mathrm{E}-8 / \mathrm{h}(30) \\
{[9]}\end{array}$ \\
\hline Plugs & & & & $\begin{array}{l}5.0 \mathrm{E}-8 / \mathrm{h}(10) \\
{[10]}\end{array}$ \\
\hline $\begin{array}{l}\text { Vessel } \\
\text { Tank (Unpressurized) } \\
\text { Leakage (extemal) }\end{array}$ & $\begin{array}{l}7.3 \mathrm{E}-7 / \mathrm{h}(10) \\
{[1]}\end{array}$ & $2.2 \mathrm{E}-8 / \mathrm{h}(19)$ & $1.3 \mathrm{E}-6 / \mathrm{h}(30)$ & $\begin{array}{l}1.0 \mathrm{E}-8 / \mathrm{h}(10) \\
{[3]}\end{array}$ \\
\hline Rupture (extermal) & & $3.0 \mathrm{E}-9 / \mathrm{h}(19)$ & & $\begin{array}{l}5.0 \mathrm{E}-10 / \mathrm{h}(30) \\
{[3]}\end{array}$ \\
\hline $\begin{array}{l}\text { Tank (Pressurized) } \\
\text { Leakage (exiemal) }\end{array}$ & & $1.0 \mathrm{E}-6 / \mathrm{h}(24)$ & $1.3 \mathrm{E}-7 / \mathrm{h}(30)$ & $\begin{array}{l}1.0 \mathrm{E}-8 / \mathrm{h}(10) \\
{[3]}\end{array}$ \\
\hline Ruppare (external) & & $4.5 \mathrm{E}-8 / \mathrm{h}(23)$ & & $\begin{array}{l}5.0 \mathrm{E}-10 / \mathrm{h}(30) \\
{[3]}\end{array}$ \\
\hline $\begin{array}{l}\text { Flange/Gasket } \\
\text { Leakage (extemal) }\end{array}$ & & $2.1 \mathrm{E}-8 / \mathrm{h}(11)$ & & $\begin{array}{l}1.0 \mathrm{E}-8 / \mathrm{h}(10) \\
{[3]}\end{array}$ \\
\hline Rupure (external) & & $1.8 \mathrm{E}-10 / \mathrm{h}(11)$ & & $\begin{array}{l}1.0 \mathrm{E}-10 / \mathrm{h}(30) \\
{[3]}\end{array}$ \\
\hline $\begin{array}{l}\text { Heat Exchanger } \\
\text { Shell/Tube } \\
\text { Fouling (ubes) }\end{array}$ & & & $\begin{array}{l}9.4 \mathrm{E}-6 / \mathrm{h}(10) \\
{[1]}\end{array}$ & $\begin{array}{l}1.0 \mathrm{E}-7 / \mathrm{h}(10) \\
{[11]}\end{array}$ \\
\hline Plugs (nubes) & $\begin{array}{l}2.6 \mathrm{E}-7 / \mathrm{h}(10) \\
{[1]}\end{array}$ & & [1] & $\begin{array}{l}3.0 \mathrm{E}-8 / \mathrm{h}(10) \\
{[12]}\end{array}$ \\
\hline Leakage (ubes) & $1.3 \mathrm{E}-6 / \mathrm{h}(10)$ & $1.8 \mathrm{E}-7 / \mathrm{h}(17)$ & [1] & $1.0 \mathrm{E}-7 / \mathrm{h}(10)$ \\
\hline Ruppure (tubes) & & $7.8 \mathrm{E}-9 / \mathrm{h}(10)$ & & $\begin{array}{l}5.0 \mathrm{E}-9 / \mathrm{h}(30) \\
{[3]}\end{array}$ \\
\hline Leakage (shell) & $\begin{array}{l}3.1 \mathrm{E}-7 / \mathrm{h}(10) \\
{[1]}\end{array}$ & $7.8 \mathrm{E}-9 / \mathrm{h}(10)$ & & $\begin{array}{l}1.0 \mathrm{E}-8 / \mathrm{h}(10) \\
{[3]}\end{array}$ \\
\hline Rupture (shell) & & $1.6 \mathrm{E}-9 / \mathrm{h}(10)$ & & $\begin{array}{l}5.0 \mathrm{E}-10 / \mathrm{h}(30) \\
{[3]}\end{array}$ \\
\hline $\begin{array}{l}\text { Heater (Electrical) } \\
\text { Fails to heat }\end{array}$ & & $1.8 \mathrm{E}-6 / \mathrm{h}(10)$ & & $1.0 \mathrm{E}-6 / \mathrm{h}(10)$ \\
\hline
\end{tabular}


WSRC-TR-93-262

\begin{tabular}{|c|c|c|c|c|}
\hline \multirow[t]{2}{*}{ System/Componen/Failure Moc's } & \multicolumn{4}{|c|}{ Aggregated and Recommended Failure Rates ${ }^{\mathbf{a}}$} \\
\hline & Calegory 1 & Category 2 & Category 3 & Recommended $^{b}$ \\
\hline Overheats & & 3.0E-7/h(10) & & $3.0 \mathrm{E}-7 / \mathrm{h}(10)$ \\
\hline Leakage (external) & & & & $\begin{array}{l}1.0 \mathrm{E}-7 / \mathrm{h}(10) \\
{[13]}\end{array}$ \\
\hline Rupture (external) & & & & $\begin{array}{l}5.0 \mathrm{E}-9 / \mathrm{h}(30) \\
{[13]}\end{array}$ \\
\hline $\begin{array}{l}\text { Strainer/Filter } \\
\text { Plugs }\end{array}$ & $4.0 \mathrm{E}-6 / \mathrm{h}(10)$ & & $\begin{array}{l}2.8 \mathrm{E}-6 / \mathrm{h}(3.0) \\
{[1]}\end{array}$ & $3.0 \mathrm{E}-6 / \mathrm{h}(10)$ \\
\hline Leakage (internal) & & $2.2 \mathrm{E}-6 / \mathrm{h}(10)$ & [1] & $3.0 \mathrm{E}-6 / \mathrm{h}(10)$ \\
\hline Rupturc (internal) & & $6.8 \mathrm{E}-7 / \mathrm{h}(10)$ & [1] & $5.0 \mathrm{E}-7 / \mathrm{h}(10)$ \\
\hline $\begin{array}{l}\text { Orifice } \\
\text { Plugs }\end{array}$ & & & $8.0 \mathrm{E}-7 / \mathrm{h}(3)$ & $1.0 \mathrm{E}-6 / \mathrm{h}(3)$ \\
\hline $\begin{array}{l}\text { Miscellaneous } \\
\text { Travelling Screen } \\
\text { Plugs }\end{array}$ & $\begin{array}{l}5.2 \mathrm{E}-7 / \mathrm{h}(10) \\
{[1]}\end{array}$ & & & $5.0 \mathrm{E}-7 / \mathrm{h}(10)$ \\
\hline
\end{tabular}

Table 6a. Summary of results for water system.

a. Aggregated failure rates are meen and error factor (in parentheses). The error factor is the 95 th percentiles:50th percentile.

b. For the recommended failure rate, the Category 1 result was used if available. If not, then the Category 2 result was used. If no Category 1 or 2 results were available, the Category 3 result was used. (Exceptions to this are indicated in the notes.) Mean values were rounded to 1, 3, or 5 times the appropriate power of 10. Error factors were rounded to 3, 5, 10, or 30.

Notes

Catesery 1

1. Failure rate based on zero failures and may be conservatively high.

\section{Caterory 2}

1. Failure rate based on zero failures and may be conservatively high.

\section{Category 3}

1. Failure modes not indicated. 


\section{Recommended}

1. Data for manual, motor-operated, and air-operated valves combined.

2. Data for manual, check, motor-operated, and air-operated valves combined. All failures assumed to be leakage. Rupture value determined using the same partition used for external rupture.

3. Results from EGG-SSRE-9639 (Category 2 data) used. These results are believed to be the most comprehensive and consistent set of data available. Rupture values for non-PCS system used. Rupture defined as $>50 \mathrm{gpm}$.

4. Assumed to be the same as fails to open.

5. Assumed to be $\mathbf{1 0}$ times better than air-operated valve.

6. Control valve data for chemical process and compressed gas systems indicate approximately $3.0 \mathrm{E}-6 / \mathrm{h}$ for fails open, fails shut, and fails to respond.

7. Trend seen for motor-driven purnp applied.

8. WASH-1400 indicates plugs is similar to extemal rupure in failure rate.

9. Flange/gasket values multiplied by 100 .

10. Valve plugs value used.

Table 6a. Summary of resuls for water system.

11. Data for chemical process system (Table 6b) indicate fouling failure rate is similar to tube leakage.

12. Category 1 results indicate mbe plugging is one-fifth as frequent as tube leakage.

13. Vessel failure rates multiplied by $\mathbf{1 0}$. 


\begin{tabular}{|c|c|c|c|c|}
\hline \multirow[t]{2}{*}{ System/Component/Failure Mode } & \multicolumn{4}{|c|}{ Aggregated and Recommended Failure Rates ${ }^{\mathbf{a}}$} \\
\hline & Category 1 & Category 2 & Category 3 & Recommended ${ }^{t}$ \\
\hline $\begin{array}{l}\text { Chemical Process } \\
\text { Valve (Standby or Safety) } \\
\text { Manual } \\
\text { Fails to open/close }\end{array}$ & & & $2.9 \mathrm{E}-4 / \mathrm{d}(8.7)$ & $\begin{array}{l}3.0 \mathrm{E}-4 / \mathrm{d}(10) \\
{[1]}\end{array}$ \\
\hline Plugs & & & & $5.0 \mathrm{E}-8 \mathrm{~h}(10)$ \\
\hline Leakage (intemal) & & & & $\begin{array}{l}1.0 \mathrm{E}-6 / \mathrm{h}(10) \\
\text { [1] }\end{array}$ \\
\hline Rupture (internal) & & & & $\begin{array}{l}5.0 \mathrm{E}-8 / \mathrm{h}(30) \\
{[1]}\end{array}$ \\
\hline Leakage (external) & & & & $\begin{array}{l}5.0 \mathrm{E}-7 / \mathrm{h}(10) \\
{[4]}\end{array}$ \\
\hline Rupture (extemal) & & & & $\begin{array}{l}3.0 \mathrm{E}-8 / \mathrm{h}(30) \\
{[4]}\end{array}$ \\
\hline $\begin{array}{l}\text { Check } \\
\text { Fails to open }\end{array}$ & & & $1.5 \mathrm{E}-4 / \mathrm{d}(3.2)$ & $\begin{array}{l}5.0 \mathrm{E}-5 / \mathrm{d}(10) \\
11]\end{array}$ \\
\hline Fails to close & & & $2.2 \mathrm{E}-3 / \mathrm{d}(4.9)$ & $\begin{array}{l}1.0 \mathrm{E}-3 / \mathrm{d}(10) \\
i 1]\end{array}$ \\
\hline Plugs & & & & $\begin{array}{l}5.0 \mathrm{E}-8 / \mathrm{h}(10) \\
{[1]}\end{array}$ \\
\hline Leakage (internal) & & & & $\begin{array}{l}1.0 \mathrm{E}-6 / \mathrm{h}(10) \\
{[1]}\end{array}$ \\
\hline Rupaure (internal) & & & & $\begin{array}{l}5.0 \mathrm{E}-8 \mathrm{~h}(30) \\
\text { [1] }\end{array}$ \\
\hline Leakage (external) & & & & $\begin{array}{l}5.0 \mathrm{E}-7 / \mathrm{h}(10) \\
{[4]}\end{array}$ \\
\hline Rupture (extemal) & & & & $\begin{array}{l}3.0 \mathrm{E}-8 / \mathrm{h}(30) \\
{[4]}\end{array}$ \\
\hline $\begin{array}{l}\text { Motor-Operated } \\
\text { Fails to open/close }\end{array}$ & & & $5.6 \mathrm{E}-3 / \mathrm{d}(6.1)$ & $\begin{array}{l}3.0 \mathrm{E}-3 / \mathrm{d}(10) \\
{[1]}\end{array}$ \\
\hline Spurious operation & & & $1.4 \mathrm{E}-6 / \mathrm{h}(4.0)$ & $\begin{array}{l}3.0 \mathrm{E}-7 / \mathrm{h}(10) \\
{[1]}\end{array}$ \\
\hline Plugs & & & & $\begin{array}{l}5.0 \mathrm{E}-8 / \mathrm{h}(10) \\
{[1]}\end{array}$ \\
\hline Leakage (internal) & & & & $\begin{array}{l}1.0 \mathrm{E}-6 / \mathrm{h}(10) \\
{[1]}\end{array}$ \\
\hline Ruppure (internal) & & & & $\begin{array}{l}5.0 \mathrm{E}-8 / \mathrm{h}(10) \\
{[1]}\end{array}$ \\
\hline
\end{tabular}




\begin{tabular}{|c|c|c|c|c|}
\hline \multirow[t]{2}{*}{ System/Component/Failure Mode } & \multicolumn{4}{|c|}{ Aggregated and Recommended Failure Rates ${ }^{n}$} \\
\hline & Category 1 & Category 2 & Category 3 & Recommended $\mathbf{b}$ \\
\hline Leakage (external) & & & & $\begin{array}{l}5.0 \mathrm{E}-7 / \mathrm{h}(10) \\
{[4]}\end{array}$ \\
\hline Rupture (external) & & & & $\begin{array}{l}3.0 \mathrm{E}-8 / \mathrm{h}(30) \\
{[4]}\end{array}$ \\
\hline $\begin{array}{l}\text { Air-Operated } \\
\text { Fails to open/close }\end{array}$ & & $\begin{array}{l}1.7 \mathrm{E}-2 / \mathrm{d}(10) \\
2.0 \mathrm{E}-7 / \mathrm{h}(5)\end{array}$ & $2.2 \mathrm{E}-3 / \mathrm{d}(4.7)$ & $1.0 \mathrm{E}-3 / \mathrm{d}(30)$ \\
\hline Spurious operation & & $2.2 \mathrm{E}-8 / \mathrm{h}(10)$ & $3.6 \mathrm{E}-6 / \mathrm{h}(6.7)$ & $1.0 \mathrm{E}-6 / \mathrm{h}(10)$ \\
\hline Plugs & & $2.2 \mathrm{E}-8 / \mathrm{h}(10)$ & & $\begin{array}{l}5.0 \mathrm{E}-8 / \mathrm{h}(10) \\
{[1]}\end{array}$ \\
\hline Leakage (internal) & & $7.3 \mathrm{E}-7 / \mathrm{h}(5)$ & & $\begin{array}{l}1.0 \mathrm{E}-6 / \mathrm{h}(10) \\
{[1]}\end{array}$ \\
\hline Rupture (internal) & & $2.2 \mathrm{E}-8 / \mathrm{h}(10)$ & & $\begin{array}{l}5.0 \mathrm{E}-8 / \mathrm{h}(30) \\
{[1]}\end{array}$ \\
\hline Leakage (external) & & $4.2 \mathrm{E}-7 / \mathrm{h}(3.6)$ & & $\begin{array}{l}5.0 \mathrm{E}-7 / \mathrm{h}(10) \\
{[4]}\end{array}$ \\
\hline Rupture (external) & & $1.8 \mathrm{E}-8 / \mathrm{h}(10)$ & & $\begin{array}{l}3.0 \mathrm{E}-8 / \mathrm{h}(30) \\
{[4]}\end{array}$ \\
\hline $\begin{array}{l}\text { Solenoid-Operated } \\
\text { Fails to open/close }\end{array}$ & & & $2.8 \mathrm{E}-3 / \mathrm{d}(5.5)$ & $\begin{array}{l}1.0 \mathrm{E}-3 / \mathrm{d}(10) \\
{[1]}\end{array}$ \\
\hline Spurious operation & & & $4.1 \mathrm{E}-7 / \mathrm{h}(3.0)$ & $\begin{array}{l}5.0 \mathrm{E}-7 / \mathrm{h}(10) \\
{[1]}\end{array}$ \\
\hline Plugs & & & & $\begin{array}{l}5.0 \mathrm{E}-8 / \mathrm{h}(10) \\
{[1]}\end{array}$ \\
\hline Leakage (internal) & & & & $1.0 \mathrm{E}-6 / \mathrm{h}(10)$ \\
\hline Ruphure (internal) & & & & $\begin{array}{l}5.0 \mathrm{E}-8 /(30) \\
{[1]}\end{array}$ \\
\hline Leakage (external) & & & & $5.0 \mathrm{E}-7 / \mathrm{h}(10)$ \\
\hline Rupture (external) & & & & $\begin{array}{l}3.0 \mathrm{E}-8 / \mathrm{h}(30) \\
{[4]}\end{array}$ \\
\hline $\begin{array}{l}\text { Safety/Relief } \\
\text { Fails to open }\end{array}$ & & & $7.8 \mathrm{E}-4 / \mathrm{d}(18)$ & $\begin{array}{l}3.0 \mathrm{E}-3 / \mathrm{d}(10) \\
{[1]}\end{array}$ \\
\hline Fails to reclose & & & $5.1 \mathrm{E}-3 / \mathrm{d}(12)$ & $\begin{array}{l}3.0 \mathrm{E}-3 / \mathrm{d}(10) \\
{[1]}\end{array}$ \\
\hline
\end{tabular}




\begin{tabular}{|c|c|c|c|c|}
\hline \multirow[t]{2}{*}{ System/Component/Failure Mode } & \multicolumn{4}{|c|}{ Aggregated and Recommended Failure Rates ${ }^{a}$} \\
\hline & Category 1 & Category 2 & Category 3 & Recommended $^{b}$ \\
\hline Leakage (intemal) & & & & $\begin{array}{l}1.0 \mathrm{E}-6 / \mathrm{h}(10) \\
{[1]}\end{array}$ \\
\hline Rupure (internal) & & & & $\begin{array}{l}5.0 \mathrm{E}-8 / \mathrm{h}(30) \\
{[1]}\end{array}$ \\
\hline Leakage (extemal) & & & & $\begin{array}{l}5.0 \mathrm{E}-7 / \mathrm{h}(10) \\
{[4]}\end{array}$ \\
\hline Rupture (external) & & & & $\begin{array}{l}3.0 \mathrm{E}-8 / \mathrm{h}(30) \\
{[4]}\end{array}$ \\
\hline $\begin{array}{l}\text { Vacurum-Breaker } \\
\text { Fails to open }\end{array}$ & & & & 1.0E-2/d(10) \\
\hline Fails to reclose & & & & $1.0 \mathrm{E}-2 / \mathrm{d}(10)$ \\
\hline Leakage (internal) & & & & $\begin{array}{l}1.0 \mathrm{E}-6 / \mathrm{h}(10) \\
\text { [1] }\end{array}$ \\
\hline Rupure (internal) & & & & $\begin{array}{l}5.0 \mathrm{E}-8 / \mathrm{h}(30) \\
{[1]}\end{array}$ \\
\hline Leakage (extemal) & & & & $\begin{array}{l}5.0 \mathrm{E}-7 / \mathrm{h}(10) \\
{[4]}\end{array}$ \\
\hline Rupture (extemal) & & & & $\begin{array}{l}3.0 \mathrm{E}-8 / \mathrm{h}(30) \\
{[4]}\end{array}$ \\
\hline $\begin{array}{l}\text { Explosive } \\
\text { Fails to open }\end{array}$ & & & & (1) \\
\hline Leakage (intemal) & & & & $\begin{array}{l}1.0 \mathrm{E}-6 / \mathrm{h}(10) \\
\text { [1] }\end{array}$ \\
\hline Rupture (intermal) & & & & $\begin{array}{l}5.0 \mathrm{E}-8 / \mathrm{h}(10) \\
\text { [1] }\end{array}$ \\
\hline Leakage (external) & & & & $5.0 \mathrm{E}-7 / \mathrm{h}(10)$ \\
\hline Rupture (external) & & & & $\begin{array}{l}3.0 \mathrm{E}-8 / \mathrm{h}(30) \\
{[4]}\end{array}$ \\
\hline $\begin{array}{l}\text { Valve (Control) } \\
\text { Motor-Operated } \\
\text { Fails open }\end{array}$ & & & & $\begin{array}{l}3.0 \mathrm{E}-6 \mathrm{~h}(10) \\
\text { [1] }\end{array}$ \\
\hline Fails closed & & & & $\begin{array}{l}3.0 \mathrm{E}-6 / \mathrm{h}(10) \\
\text { [1] }\end{array}$ \\
\hline Fails to respond & & & & $\begin{array}{l}3.0 \mathrm{E}-6 / \mathrm{h}(10) \\
\text { [1] }\end{array}$ \\
\hline
\end{tabular}


Table 6b. Summary of results for chemical process system.

\begin{tabular}{|c|c|c|c|c|}
\hline \multirow[t]{2}{*}{ System/Componen//Failure Mode } & \multicolumn{4}{|c|}{ Aggregated and Recommended Failure Rates ${ }^{a}$} \\
\hline & Category 1 & Category 2 & Category 3 & Recommended $\mathrm{b}$ \\
\hline Plugs & & & & $\begin{array}{l}5.0 \mathrm{E}-8 / \mathrm{h}(10) \\
{[1]}\end{array}$ \\
\hline Leakage (extemal) & & & & $5.0 \mathrm{E}-7 \mathrm{~h}(10)$ \\
\hline Rupture (external) & & & & $\begin{array}{l}3.0 \mathrm{E}-8 / \mathrm{h}(30) \\
{[4]}\end{array}$ \\
\hline $\begin{array}{l}\text { Air-Operated } \\
\text { Fails open }\end{array}$ & & $6.0 \mathrm{E}-6 / \mathrm{h}(10)$ & $\begin{array}{l}2.7 \mathrm{E}-5 / \mathrm{h} \\
{[1]}\end{array}$ & $3.0 \mathrm{E}-6 / \mathrm{h}(10)$ \\
\hline Fails closed & & $2.6 \mathrm{E}-6 / \mathrm{h}(10)$ & [1] & $3.0 \mathrm{E}-6 / \mathrm{h}(10)$ \\
\hline Fails to respond & & & {$[1]$} & $\begin{array}{l}3.0 \mathrm{E}-6 / \mathrm{h}(10) \\
{[1]}\end{array}$ \\
\hline Plugs & & & & $\begin{array}{l}5.0 \mathrm{E}-8 \mathrm{~h}(10) \\
\text { [1] }\end{array}$ \\
\hline Leakage (external) & & $1.1 \mathrm{E}-5 / \mathrm{h}(10)$ & & $\begin{array}{l}5.0 \mathrm{E}-7 \mathrm{~h}(10) \\
{[4]}\end{array}$ \\
\hline Rupture (external) & & & & $\begin{array}{l}3.0 \mathrm{E}-8 \ln (30) \\
{[4]}\end{array}$ \\
\hline $\begin{array}{l}\text { Solenoid-Operated } \\
\text { Fails open }\end{array}$ & & & & $\begin{array}{l}3.0 \mathrm{E}-6 / \mathrm{h}(10) \\
{[1]}\end{array}$ \\
\hline Fails closed & & & & $\begin{array}{l}3.0 \mathrm{E}-6 / \mathrm{h}(10) \\
\text { [1] }\end{array}$ \\
\hline Fails to respond & & & & $\begin{array}{l}3.0 \mathrm{E}-6 / \mathrm{h}(10) \\
\text { [1] }\end{array}$ \\
\hline Plugs & & & & $5.0 E-8 / \mathrm{h}(10)$ \\
\hline Leakage (external) & & & & $\begin{array}{l}5.0 \mathrm{E}-7 / \mathrm{h}(10) \\
{[4]}\end{array}$ \\
\hline Rupture (external) & & & & $\begin{array}{l}3.0 \mathrm{E}-8 / \mathrm{h}(30) \\
{[4]}\end{array}$ \\
\hline $\begin{array}{l}\text { Pump } \\
\text { Motor-Driven } \\
\text { Fails to start }\end{array}$ & & $\begin{array}{l}8.1 \mathrm{E}-3 / \mathrm{d}(10) \\
8.9 \mathrm{E}-6 / \mathrm{h}(4.1)\end{array}$ & $\begin{array}{l}1.6 \mathrm{E}-2 / \mathrm{d}(8.1) \text {, } \\
1.5 \mathrm{E}-5 / \mathrm{h}\end{array}$ & $\begin{array}{l}1.0 \mathrm{E}-2 / \mathrm{d}(10) \\
{[2]}\end{array}$ \\
\hline Fails to run & & $5.8 \mathrm{E}-4 / \mathrm{h}(12)$ & $6.0 \mathrm{E}-5 / \mathrm{h}(11)$ & {$[2]$} \\
\hline Overspeed & & & & $\begin{array}{l}3.0 \mathrm{E}-5 / \mathrm{h}(10) \\
{[2]}\end{array}$ \\
\hline
\end{tabular}




\begin{tabular}{|c|c|c|c|c|}
\hline \multirow[t]{2}{*}{ System/Component/Failure Mode } & \multicolumn{4}{|c|}{ Aggregated and Recommended Failure Rates ${ }^{\mathrm{a}}$} \\
\hline & Category 1 & Category 2 & Category 3 & Recommended ${ }^{b}$ \\
\hline Fails to stop & & & & $\begin{array}{l}1.0 \mathrm{E}-2 / \mathrm{d}(10) \\
{[2]}\end{array}$ \\
\hline Leakage (extemal) & & $9.2 \mathrm{E}-6 / \mathrm{h}(5.4)$ & & $1.0 \mathrm{E}-6 / \mathrm{h}(10)$ \\
\hline Rupture (external) & & $\begin{array}{l}5.2 \mathrm{E}-8 / \mathrm{h}(10) \\
{[1]}\end{array}$ & & $\begin{array}{l}5.0 \mathrm{E}-8 / \mathrm{h}(30) \\
{[4]}\end{array}$ \\
\hline $\begin{array}{l}\text { Turbine-Driven } \\
\text { Fails to stant }\end{array}$ & & & $2.6 \mathrm{E}-2 / \mathrm{d}(4.3)$ & $\begin{array}{l}3.0 \mathrm{E}-2 / \mathrm{d}(10) \\
{[1]}\end{array}$ \\
\hline Fails to run & & & $8.9 \mathrm{E}-5 / \mathrm{h}(5.0)$ & $\begin{array}{l}1.0 \mathrm{E}-4 / \mathrm{h}(30) \\
{[1]}\end{array}$ \\
\hline Overspeed & & & & $\begin{array}{l}3.0 \mathrm{E}-5 / \mathrm{h}(10) \\
{[1]}\end{array}$ \\
\hline Fails to stop & & & & $\begin{array}{l}3.0 \mathrm{E}-2 / \mathrm{d}(10) \\
11]\end{array}$ \\
\hline Leakage (external) & & & & $\begin{array}{l}1.0 \mathrm{E}-6 / \mathrm{h}(10) \\
{[4]}\end{array}$ \\
\hline Rupuire (external) & & & & $\begin{array}{l}5.0 \mathrm{E}-8 / \mathrm{h}(30) \\
{[4]}\end{array}$ \\
\hline $\begin{array}{l}\text { Diesel-Driven } \\
\text { Fails sts start }\end{array}$ & & & & $1.0 \mathrm{E}-2 / \mathrm{d}(10)$ \\
\hline Fails to run & & & & $\begin{array}{l}5.0 \mathrm{E}-3 / \mathrm{h}(10) \\
{[1]}\end{array}$ \\
\hline Overspeed & & & & [1] \\
\hline Fails to stop & & & & $1.0 \mathrm{E}-2 / \mathrm{d}(10)$ \\
\hline Leakage (external) & & & & $\begin{array}{l}1.0 \mathrm{E}-6 / \mathrm{h}(10) \\
{[4]}\end{array}$ \\
\hline Rupture (external) & & & & $\begin{array}{l}5.0 \mathrm{E}-8 / \mathrm{h}(30) \\
{[4]}\end{array}$ \\
\hline $\begin{array}{l}\text { Piping/Hose/Jumper } \\
\text { Piping } \\
\text { Leakage (external) }\end{array}$ & & & & $\begin{array}{l}3.0 \mathrm{E}-9 / \mathrm{h}-\mathrm{ft}(10) \\
{[1]}\end{array}$ \\
\hline Ruphure (external) & & & $5.1 \mathrm{E}-12 \mathrm{~h}-\mathrm{ft}(15)$ & $\begin{array}{l}1.0 \mathrm{E}-10 / \mathrm{h}-\mathrm{ft}(30) \\
{[1]}\end{array}$ \\
\hline Plugs & & & & $\begin{array}{l}1.0 \mathrm{E}-10 / \mathrm{h}-\mathrm{ft}(30) \\
{[1]}\end{array}$ \\
\hline
\end{tabular}




\begin{tabular}{|c|c|c|c|c|}
\hline \multirow[t]{2}{*}{ System/Component/Failure Mode } & \multicolumn{4}{|c|}{ Aggregated and Recommended Failure Rates ${ }^{a}$} \\
\hline & Category 1 & Category 2 & Category 3 & Recommended $^{b}$ \\
\hline $\begin{array}{l}\text { Hose } \\
\text { Leakage (extemal) }\end{array}$ & & & & $\begin{array}{l}1.0 \mathrm{E}-9 / \mathrm{h}-\mathrm{ft}(10) \\
\text { [1] }\end{array}$ \\
\hline Rupture (external) & & & $2.9 \mathrm{E}-8 / \mathrm{h}-\mathrm{ft}(15)$ & $1.0 \mathrm{E}-8 / \mathrm{h}-\mathrm{ft}(10)$ \\
\hline Plugs & & & & $1.0 \mathrm{E}-8 / \mathrm{h}-\mathrm{ft}(10)$ \\
\hline $\begin{array}{l}\text { Jumper } \\
\text { Leakage (external) }\end{array}$ & & & & $\begin{array}{l}1.0 \mathrm{E}-6 / \mathrm{h}(10) \\
{[1]}\end{array}$ \\
\hline Rupture (external) & & & & $\begin{array}{l}1.0 \mathrm{E}-8 \mathrm{~h}(30) \\
{[1]}\end{array}$ \\
\hline Plugs & & & & $\begin{array}{l}5.0 \mathrm{E}-8 / \mathrm{h}(10) \\
{[1]}\end{array}$ \\
\hline $\begin{array}{l}\text { Vessel } \\
\text { Tank (Unpressurized) } \\
\text { Leakage (external) }\end{array}$ & & $2.6 \mathrm{E}-6 / \mathrm{h}(10)$ & $1.2 \mathrm{E}-6 / \mathrm{h}(9.3)$ & $\begin{array}{l}1.0 \mathrm{E}-7 / \mathrm{h}(10) \\
{[3]}\end{array}$ \\
\hline Rupure (external) & & & & $\begin{array}{l}5.0 \mathrm{E}-9 / \mathrm{h}(30) \\
{[3]}\end{array}$ \\
\hline $\begin{array}{l}\text { Tank (Pressurized) } \\
\text { Leakage (external) }\end{array}$ & & $3.7 \mathrm{E}-6 / \mathrm{h}(3.8)$ & $1.1 \mathrm{E}-8 / \mathrm{h}(17)$ & $\begin{array}{l}1.0 \mathrm{E}-7 / \mathrm{h}(10) \\
{[3]}\end{array}$ \\
\hline Rupture (extemal) & & $3.8 \mathrm{E}-7 / \mathrm{h}(10)$ & & $\begin{array}{l}5.0 \mathrm{E}-9 / \mathrm{h}(30) \\
{[3]}\end{array}$ \\
\hline $\begin{array}{l}\text { Flange/Gasket } \\
\text { Leakage (external) }\end{array}$ & & & & $\begin{array}{l}1.0 \mathrm{E}-7 / \mathrm{h}(10) \\
{[3]}\end{array}$ \\
\hline Rupture (external) & & & & $\begin{array}{l}1.0 \mathrm{E}-9 / \mathrm{h}(10) \\
{[3]}\end{array}$ \\
\hline $\begin{array}{l}\text { Heat Exchanger } \\
\text { Shell/Tube } \\
\text { Fouling (ubes) }\end{array}$ & & $7.8 \mathrm{E}-6 / \mathrm{h}(1.3)$ & & $\begin{array}{l}1.0 \mathrm{E}-6 / \mathrm{h}(10) \\
{[3]}\end{array}$ \\
\hline Plugs (nubes) & & & & $\begin{array}{l}3.0 \mathrm{E}-7 / \mathrm{h}(10) \\
{[3]}\end{array}$ \\
\hline Leakage (ubes) & & $7.7 \mathrm{E}-6 / \mathrm{h}(1.3)$ & $2.6 \mathrm{E}-5 / \mathrm{h}(7.5)$ & $\begin{array}{l}1.0 \mathrm{E}-6 / \mathrm{h}(10) \\
{[3]}\end{array}$ \\
\hline Rupare (tubes) & & & & $\begin{array}{l}5.0 \mathrm{E}-8 / \mathrm{h}(30) \\
{[3]}\end{array}$ \\
\hline Leakage (shell) & & & & $\begin{array}{l}1.0 \mathrm{E}-7 / \mathrm{h}(10) \\
3]\end{array}$ \\
\hline
\end{tabular}


WSRC-TR-93-262

\begin{tabular}{|c|c|c|c|c|}
\hline \multirow[t]{2}{*}{ System/Component/Failure Mode } & \multicolumn{4}{|c|}{ Aggregated and Recommended Failure Rates ${ }^{\mathrm{a}}$} \\
\hline & Category 1 & Category 2 & Category 3 & Recommended $^{b}$ \\
\hline Rupture (shell) & & & & $\begin{array}{l}5.0 \mathrm{E}-9 \mathrm{~h}(30) \\
{[3]}\end{array}$ \\
\hline $\begin{array}{l}\text { Heater (Electrical) } \\
\text { Fails to heat }\end{array}$ & & $3.5 \mathrm{E}-5 / \mathrm{h}(5.8)$ & & $\begin{array}{l}1.0 \mathrm{E}-5 / \mathrm{h}(10) \\
{[3]}\end{array}$ \\
\hline Overheats & & $7.0 \mathrm{E}-6 / \mathrm{h}(10)$ & & $\begin{array}{l}3.0 \mathrm{E}-6 / \mathrm{h}(10) \\
{[3]}\end{array}$ \\
\hline Leakage (external) & & & & $\begin{array}{l}1.0 \mathrm{E}-6 / \mathrm{h}(10) \\
{[3]}\end{array}$ \\
\hline Ruptur: (external) & & & & $\begin{array}{l}5.0 \mathrm{E}-8 / \mathrm{h}(30) \\
{[3]}\end{array}$ \\
\hline $\begin{array}{l}\text { Strainer//Filter } \\
\text { Plugs }\end{array}$ & & $3.1 \mathrm{E}-6 / \mathrm{h}(11)$ & $\begin{array}{l}5.6 \mathrm{E}-6 / \mathrm{h}(5.0) \\
{[1]}\end{array}$ & $\begin{array}{l}3.0 \mathrm{E}-6 / \mathrm{h}(10) \\
{[1]}\end{array}$ \\
\hline Leakage (internal) & & $7.0 \mathrm{E}-6 / \mathrm{h}(11)$ & [1] & $\begin{array}{l}3.0 \mathrm{E}-6 / \mathrm{h}(10) \\
{[1]}\end{array}$ \\
\hline Rupture (internal) & & $\begin{array}{l}7.0 \mathrm{E}-7 / \mathrm{h}(11) \\
\text { [1] }\end{array}$ & [1] & $\begin{array}{l}5.0 \mathrm{E}-7 \mathrm{~h}(10) \\
{[1]}\end{array}$ \\
\hline $\begin{array}{c}\text { Orifice } \\
\text { Plugs }\end{array}$ & & & & $\begin{array}{l}1.0 \mathrm{E}-6 / \mathrm{h}(10) \\
{[1]}\end{array}$ \\
\hline $\begin{array}{l}\text { Miscellaneous } \\
\text { Mixer/Blender } \\
\text { Faihure } \\
\end{array}$ & & & & $\begin{array}{l}5.0 \mathrm{E}-6 / \mathrm{h}(10) \\
{[5]}\end{array}$ \\
\hline $\begin{array}{c}\text { Agitator } \\
\text { Failure }\end{array}$ & & $6.5 \mathrm{E}-6 / \mathrm{h}(10)$ & $2.5 \mathrm{E}-5 / \mathrm{h}(10)$ & $5.0 \mathrm{E}-6 / \mathrm{h}(10)$ \\
\hline $\begin{array}{l}\text { Cenvifuge } \\
\text { Failure }\end{array}$ & & & & $\begin{array}{l}5.0 \mathrm{E}-6 / \mathrm{h}(10) \\
{[5]}\end{array}$ \\
\hline
\end{tabular}

Table 6b. Summary of results for chemical process system.

2. Aggregited failure rates are mean and error factor (in parentheses). The error factor is the 95 th percentile/50th percentile.

b. For the recommended failure rate, the Category 1 result was used if available. If not, then the Category 2 result was used. If no Category 1 or 2 results were available, the Category 3 result was used. (Exceptions to this are indicated in the notes.) Mean values were rounded to 1, 3, or 5 times the appropriate power of 10. Error factors were rounded to 3,5, 10, or 30. 


\section{Notes}

\section{Catcrory 2}

1. Failure rate based on zero failure and may be conservatively high.

\section{Categery 3}

1. Failure modes not indicated.

\section{Recommended}

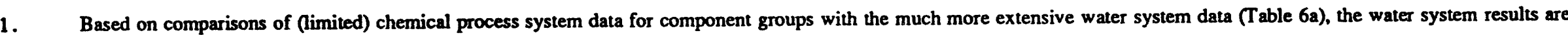
appropriate.

2. Based on comparisons of (limited) chemical process system data for componemultiplied by 3 .

3. Based on comparisons of (limited) chemical process system data for compone. multipliea by 10 .

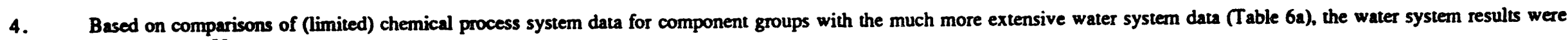
multiplied by 50 .

5. Agitator results were used.

with the much more extensive water system data (Table 6a), the water system results were

with the much more extensive water system dau (Table 6a), the water system results were 


\begin{tabular}{|c|c|c|c|c|}
\hline \multirow[t]{2}{*}{ System/Component/Failure Mode } & \multicolumn{4}{|c|}{ Failure Data (Category 1 Sources) } \\
\hline & Category 1 & Category 2 & Category 3 & Recommended $^{\mathrm{b}}$ \\
\hline $\begin{array}{l}\text { Compressed Gas } \\
\text { Valve (Standby or Safery) } \\
\text { Manual } \\
\text { Fails to open/close }\end{array}$ & & $\begin{array}{l}2.2 \mathrm{E}-3 / \mathrm{d}(10), \\
6.0 \mathrm{E}-7 / \mathrm{h}(10)\end{array}$ & & $\begin{array}{l}1.0 \mathrm{E}-3 / \mathrm{d}(10) \\
{[2]}\end{array}$ \\
\hline Plugs & & & & $\begin{array}{l}5.0 \mathrm{E}-7 / \mathrm{h}(10) \\
{[3]}\end{array}$ \\
\hline Leakage (internal) & & & & $\begin{array}{l}1.0 \mathrm{E}-5 / \mathrm{h}(10) \\
{[3]}\end{array}$ \\
\hline Rupture (internal) & & & & $\begin{array}{l}5.0 \mathrm{E}-7 / \mathrm{h}(30) \\
{[3]}\end{array}$ \\
\hline Leakage (external) & & $1.1 \mathrm{E}-7 / \mathrm{h}(10)$ & & $\begin{array}{l}1.0 \mathrm{E}-7 / \mathrm{h}(10) \\
{[3]}\end{array}$ \\
\hline Rupture (externa) & & & & $\begin{array}{l}5.0 \mathrm{E}-9 / \mathrm{h}(30) \\
{[3]}\end{array}$ \\
\hline $\begin{array}{l}\text { Check } \\
\text { Fails to open }\end{array}$ & & $1.1 \mathrm{E}-6 / \mathrm{h}(2.7)$ & & $\begin{array}{l}1.0 \mathrm{E}-4 / \mathrm{d}(10) \\
{[2]}\end{array}$ \\
\hline Fails to close & & $2.4 \mathrm{E}-6 / \mathrm{h}(2.7)$ & & $\begin{array}{l}3.0 \mathrm{E}-3 / \mathrm{d}(10) \\
{[2]}\end{array}$ \\
\hline Plugs & & & & $\begin{array}{l}5.0 \mathrm{E}-7 / \mathrm{h}(10) \\
{[3]}\end{array}$ \\
\hline Leakage (internal) & & $8.4 \mathrm{E}-6 \mathrm{~h}(10)$ & & $\begin{array}{l}1.0 \mathrm{E}-5 / \mathrm{h}(10) \\
{[3]}\end{array}$ \\
\hline Rupkure (internal) & & $4.8 \mathrm{E}-7 / \mathrm{h}(10)$ & & $\begin{array}{l}5.0 \mathrm{E}-7 / \mathrm{h}(30) \\
{[3]}\end{array}$ \\
\hline Leakage (extemal) & & $8.4 \mathrm{E}-6 / \mathrm{h}(10)$ & & $\begin{array}{l}1.0 \mathrm{E}-7 / \mathrm{h}(10) \\
{[3]}\end{array}$ \\
\hline RuF & & $\begin{array}{l}3.1 \mathrm{E}-7 \mathrm{~h}(10) \\
\text { [1] }\end{array}$ & & $\begin{array}{l}5.0 \mathrm{E}-9 / \mathrm{h}(30) \\
{[3]}\end{array}$ \\
\hline $\begin{array}{l}\text { Motor-OF zated } \\
\text { Fails to open/close }\end{array}$ & & $5.3 \mathrm{E}-4 / \mathrm{d}(10)$ & & $1.0 \mathrm{E}-2 / \mathrm{d}(10)$ \\
\hline Spurious operation & & & & $\begin{array}{l}3.0 \mathrm{E}-7 / \mathrm{h}(10) \\
{[1]}\end{array}$ \\
\hline Plugs & & & & $\begin{array}{l}5.0 \mathrm{E}-7 / \mathrm{h}(10) \\
3]\end{array}$ \\
\hline Leakage (internal) & & & & $\begin{array}{l}1.0 \mathrm{E}-5 / \mathrm{h}(10) \\
{[3]}\end{array}$ \\
\hline Rupture (internal) & & & & $\begin{array}{l}5.0 \mathrm{E}-7 / \mathrm{h}(30) \\
{[3]}\end{array}$ \\
\hline
\end{tabular}




\begin{tabular}{|c|c|c|c|c|}
\hline \multirow[t]{2}{*}{ System/Componen/Failure Mode } & \multicolumn{4}{|c|}{ Failure Data (Category 1 Sources) ${ }^{\mathbf{a}}$} \\
\hline & Category 1 & Category 2 & Category 3 & Recommended $b$ \\
\hline Leakage (external) & & $1.5 \mathrm{E}-7 / \mathrm{h}(10)$ & & $\begin{array}{l}1.0 \mathrm{E}-7 / \mathrm{h}(10) \\
{[3]}\end{array}$ \\
\hline Rupture (external) & & & & $\begin{array}{l}5.0 \mathrm{E}-9 / \mathrm{h}(30) \\
{[3]}\end{array}$ \\
\hline $\begin{array}{l}\text { Air-Operated } \\
\text { Fails to open/close }\end{array}$ & & $3.0 \mathrm{E}-3 / \mathrm{d}(1.2)$ & & $\begin{array}{l}3.0 \mathrm{E}-3 / \mathrm{d}(30) \\
{[2]}\end{array}$ \\
\hline Spurious operation & & $1.9 \mathrm{E}-6 / \mathrm{h}(8.1)$ & & $\begin{array}{l}1.0 \mathrm{E}-6 / \mathrm{h}(10) \\
{[1]}\end{array}$ \\
\hline Plugs & & $6.5 \mathrm{E}-7 / \mathrm{h}(10)$ & & $\begin{array}{l}5.0 \mathrm{E}-7 / \mathrm{h}(10) \\
{[3]}\end{array}$ \\
\hline Leakage (intemal) & & $2.5 \mathrm{E}-6 / \mathrm{h}(2.5)$ & & $\begin{array}{l}1.0 \mathrm{E}-5 / \mathrm{h}(10) \\
{[3]}\end{array}$ \\
\hline Rupture (internal) & & $4.1 \mathrm{E}-7 / \mathrm{h}(4.8)$ & & $\begin{array}{l}5.0 \mathrm{E}-7 \mathrm{~h}(30) \\
{[3]}\end{array}$ \\
\hline Leakage (extemal) & & $1.6 \mathrm{E}-6 / \mathrm{h}(5.5)$ & & $\begin{array}{l}1.0 \mathrm{E}-7 / \mathrm{h}(10) \\
{[3]}\end{array}$ \\
\hline Rupture (external) & & $\begin{array}{l}2.0 \mathrm{E}-8 / \mathrm{h}(10) \\
\text { [1] }\end{array}$ & & $\begin{array}{l}5.0 \mathrm{E}-9 / \mathrm{h}(30) \\
{[3]}\end{array}$ \\
\hline $\begin{array}{l}\text { Solenoid-Operated } \\
\text { Fails to open/close }\end{array}$ & & $6.8 \mathrm{E}-7 / \mathrm{h}(10)$ & & $\begin{array}{l}3.0 \mathrm{E}-3 / \mathrm{d}(10) \\
{[2]}\end{array}$ \\
\hline Spurious operation & & $4.2 \mathrm{E}-7 / \mathrm{h}(10)$ & & $\begin{array}{l}5.0 \mathrm{E}-7 / \mathrm{h}(10) \\
{[1]}\end{array}$ \\
\hline Plugs & & & & $\begin{array}{l}5.0 \mathrm{E}-7 / \mathrm{h}(10) \\
{[3]}\end{array}$ \\
\hline Leakage (intemal) & & & & $1.0 \mathrm{E}-5 / \mathrm{h}(10)$ \\
\hline Rupture (internal) & & & & $\begin{array}{l}5.0 \mathrm{E}-7 / \mathrm{h}(30) \\
{[3]}\end{array}$ \\
\hline Leakage (extemal) & & $6.8 \mathrm{E}-6 / \mathrm{h}(10)$ & & $\begin{array}{l}1.0 \mathrm{E}-7 / \mathrm{h}(10) \\
{[3]}\end{array}$ \\
\hline Rupture (external) & & $\begin{array}{l}2.6 \mathrm{E}-7 / \mathrm{h}(10) \\
{[1]}\end{array}$ & & $\begin{array}{l}5.0 \mathrm{E}-9 / \mathrm{h}(30) \\
{[3]}\end{array}$ \\
\hline $\begin{array}{l}\text { Safety/Relief } \\
\text { Fails to open }\end{array}$ & & & & $1.0 \mathrm{E}-2 / \mathrm{d}(10)$ \\
\hline Fails to reclose & & & & $\begin{array}{l}1.0 \mathrm{E}-2 / \mathrm{d}(10) \\
{[2]}\end{array}$ \\
\hline
\end{tabular}


WSRC-TR-93-262

\begin{tabular}{|c|c|c|c|c|}
\hline \multirow{2}{*}{ System/Component/Failure Mode } & \multicolumn{4}{|c|}{ Failure Data (Category 1 Sources) $^{\mathrm{a}}$} \\
\hline & Category 1 & Category 2 & Category 3 & Recommended $^{\mathrm{b}}$ \\
\hline Lcakage (internal) & & 9.3E-7/h(10) & & $\begin{array}{l}1.0 \mathrm{E}-5 / \mathrm{h}(10) \\
{[3]}\end{array}$ \\
\hline Rupture (internal) & & & & $\begin{array}{l}5.0 \mathrm{E}-7 / \mathrm{h}(30) \\
{[3]}\end{array}$ \\
\hline Leakage (extemal) & & 9.3E-7/h(10) & & $\begin{array}{l}1.0 \mathrm{E}-7 / \mathrm{h}(10) \\
{[3]}\end{array}$ \\
\hline Rupture (external) & & $\begin{array}{l}1.2 \mathrm{E}-7 / \mathrm{h}(10) \\
{[1]}\end{array}$ & & $\begin{array}{l}5.0 \mathrm{E}-9 / \mathrm{h}(30) \\
{[3]}\end{array}$ \\
\hline $\begin{array}{c}\text { Vactum-Breaker } \\
\text { Fails to open } \\
\end{array}$ & & & & $\begin{array}{l}3.0 \mathrm{E}-2 / \mathrm{d}(10) \\
{[2]}\end{array}$ \\
\hline Fails to reclose & & & & $\begin{array}{l}3.0 \mathrm{E}-2 / \mathrm{d}(10) \\
{[2]}\end{array}$ \\
\hline Leakage (internal) & & & & $\begin{array}{l}1.0 \mathrm{E}-5 / \mathrm{h}(10) \\
{[3]}\end{array}$ \\
\hline Rupture (internal) & & & & $\begin{array}{l}5.0 \mathrm{E}-7 / \mathrm{h}(30) \\
{[3]}\end{array}$ \\
\hline Leakage (external) & & & & $\begin{array}{l}1.0 \mathrm{E}-7 / \mathrm{h}(10) \\
{[3]}\end{array}$ \\
\hline Rupure (external) & & & & $\begin{array}{l}5.0 \mathrm{E}-9 / \mathrm{h}(30) \\
{[3]}\end{array}$ \\
\hline $\begin{array}{l}\text { Valve (Control) } \\
\text { Motor-Operated } \\
\text { Fails open }\end{array}$ & & & & $\begin{array}{l}3.0 \mathrm{E}-6 / \mathrm{h}(10) \\
{[1]}\end{array}$ \\
\hline Fails closed & & & & $\begin{array}{l}3.0 \mathrm{E}-6 / \mathrm{h}(10) \\
{[1]}\end{array}$ \\
\hline Fails to respond & & & & $\begin{array}{l}3.0 \mathrm{E}-6 / \mathrm{h}(10) \\
{[1]}\end{array}$ \\
\hline Plugs & & & & $\begin{array}{l}5.0 \mathrm{E}-7 / \mathrm{h}(10) \\
{[3]}\end{array}$ \\
\hline Leakage (extemal) & & & & $\begin{array}{l}1.0 \mathrm{E}-7 / \mathrm{h}(10) \\
{[3]}\end{array}$ \\
\hline Ruphure (external) & & & & $\begin{array}{l}5.0 \mathrm{E}-9 / \mathrm{h}(30) \\
{[3]}\end{array}$ \\
\hline $\begin{array}{l}\text { Air-Operated } \\
\text { Fails open }\end{array}$ & & $3.0 \mathrm{E}-6 / \mathrm{h}(6.1)$ & & $\begin{array}{l}3.0 \mathrm{E}-6 / \mathrm{h}(10) \\
{[1]}\end{array}$ \\
\hline Fails closed & & $3.0 \mathrm{E}-6 / \mathrm{h}(6.1)$ & & $\begin{array}{l}3.0 \mathrm{E}-6 / \mathrm{h}(10) \\
{[1]}\end{array}$ \\
\hline
\end{tabular}




\begin{tabular}{|c|c|c|c|c|}
\hline \multirow[t]{2}{*}{ System/Componen//Failure Mode } & \multicolumn{4}{|c|}{ Failure Data (Category 1 Sources) ${ }^{\mathrm{a}}$} \\
\hline & Category 1 & Category 2 & Category 3 & Recommended $\mathbf{b}$ \\
\hline Fails to respond & & $3.0 \mathrm{E}-6 / \mathrm{h}(6.1)$ & & $\begin{array}{l}3.0 \mathrm{E}-6 / \mathrm{h}(10) \\
\text { [1] }\end{array}$ \\
\hline Plugs & & & & $\begin{array}{l}5.0 \mathrm{E}-7 / \mathrm{h}(10) \\
{[3]}\end{array}$ \\
\hline Leakage (external) & & $4.9 \mathrm{E}-6 / \mathrm{h}(3.7)$ & & $1.0 \mathrm{E}-7 / \mathrm{h}(10)$ \\
\hline Rupture (external) & & $\begin{array}{l}1.4 \mathrm{E}-7 / \mathrm{h}(10) \\
\text { [1] }\end{array}$ & & $\begin{array}{l}5.0 \mathrm{E}-9 / \mathrm{h}(30) \\
{[3]}\end{array}$ \\
\hline $\begin{array}{l}\text { Solenoid-Operated } \\
\text { Fails open }\end{array}$ & & $1.6 \mathrm{E}-6 / \mathrm{d}$ & & $\begin{array}{l}3.0 \mathrm{E}-6 / \mathrm{h}(10) \\
{[1]}\end{array}$ \\
\hline Fails closed & & [2] & & $\begin{array}{l}3.0 \mathrm{E}-6 / \mathrm{h}(10) \\
\text { [i] }\end{array}$ \\
\hline Fails to respond & & [2] & & $\begin{array}{l}3.0 \mathrm{E}-6 / \mathrm{h}(10) \\
\text { [1] }\end{array}$ \\
\hline Plugs & & & & $\begin{array}{l}5.0 \mathrm{E}-7 / \mathrm{h}(10) \\
{[3]}\end{array}$ \\
\hline Leakage (external) & & & & $\begin{array}{l}1.0 \mathrm{E}-7 / \mathrm{h}(10) \\
3]\end{array}$ \\
\hline Rupture (external) & & & & $\begin{array}{l}5.0 \mathrm{E}-9 / \mathrm{h}(30) \\
{[3]}\end{array}$ \\
\hline $\begin{array}{l}\text { Compressor } \\
\text { Motor-Driven } \\
\text { Fails to start }\end{array}$ & $7.5 E-3 / d(7.1)$ & $\begin{array}{l}3.9 \mathrm{E}-5 / \mathrm{d}(10), \\
8.2 \mathrm{E}-6 / \mathrm{h}(3.6)\end{array}$ & $1.6 \mathrm{E}-4 / \mathrm{h}(30)$ & $5.0 \mathrm{E}-3 / \mathrm{d}(5)$ \\
\hline Fails to run & $5.6 \mathrm{E}-5 / \mathrm{h}(1.2)$ & $\begin{array}{l}1.3 \mathrm{E}-5 / \mathrm{h}(3.6) \\
4.1 \mathrm{E}-3 / \mathrm{h}(2.0)\end{array}$ & $1.6 \mathrm{E}-4 / \mathrm{h}(30)$ & $5.0 \mathrm{E}-5 / \mathrm{h}(3)$ \\
\hline Overspeed & & $2.6 \mathrm{E}-6 / \mathrm{h}(5)$ & & $\begin{array}{l}1.0 \mathrm{E}-5 / \mathrm{h}(5) \\
{[5]}\end{array}$ \\
\hline Fails to stop & & & & $\begin{array}{l}5.0 \mathrm{E}-3 / \mathrm{d}(5) \\
{[6]}\end{array}$ \\
\hline Leakage (external) & & $7.2 \mathrm{E}-7 \mathrm{~h}(10)$ & & $\begin{array}{l}3.0 \mathrm{E}-7 / \mathrm{h}(10) \\
{[3]}\end{array}$ \\
\hline Rupture (extemal) & & & & $1.0 \mathrm{E}-8 / \mathrm{h}(30)$ \\
\hline $\begin{array}{l}\text { Piping/Hose/Jumper/Tube } \\
\text { Piping } \\
\text { Leakage (external) }\end{array}$ & & & & $\begin{array}{l}3.0 \mathrm{E}-8 / \mathrm{h}-\mathrm{ft}(10) \\
{[3]}\end{array}$ \\
\hline
\end{tabular}




\begin{tabular}{|c|c|c|c|c|}
\hline \multirow[t]{2}{*}{ System/Component/Failure Mode } & \multicolumn{4}{|c|}{ Failure Data (Caregory 1 Sources) $^{\mathbf{a}}$} \\
\hline & Category 1 & Category 2 & Category 3 & Recommended $^{b}$ \\
\hline Rupture (external) & & & & $\begin{array}{l}1.0 \mathrm{E}-9 / \mathrm{h}-\mathrm{ft}(30) \\
{[3] \quad \text {. }}\end{array}$ \\
\hline Plugs & & & & $\begin{array}{l}1.0 \mathrm{E}-9 / \mathrm{h}-\mathrm{ft}(30) \\
{[3]}\end{array}$ \\
\hline $\begin{array}{l}\text { Hose } \\
\quad \text { Leakage (external) }\end{array}$ & & & & $\begin{array}{l}1.0 \mathrm{E}-8 / \mathrm{h}-\mathrm{ft}(10) \\
{[3]}\end{array}$ \\
\hline Rupture (external) & & & & $\begin{array}{l}1.0 \mathrm{E}-7 / \mathrm{h}-\mathrm{ft}(10) \\
{[3]}\end{array}$ \\
\hline Plugs & & & & $\begin{array}{l}1.0 \mathrm{E}-7 / \mathrm{h}-\mathrm{ft}(10) \\
{[3]}\end{array}$ \\
\hline $\begin{array}{l}\text { Jumper } \\
\text { Leakage (external) }\end{array}$ & & & & $\begin{array}{l}1.0 \mathrm{E}-5 / \mathrm{h}(10) \\
{[3]}\end{array}$ \\
\hline Rupnure (extemal) & & & & $\begin{array}{l}1.0 \mathrm{E}-7 / \mathrm{h}(30) \\
{[3]}\end{array}$ \\
\hline Flugs & & & & $\begin{array}{l}1.0 \mathrm{E}-7 / \mathrm{h}(30) \\
33]\end{array}$ \\
\hline $\begin{array}{l}\text { Tube } \\
\text { Leakage (external) }\end{array}$ & & & & $\begin{array}{l}3.0 \mathrm{E}-7 / \mathrm{h}-\mathrm{ft}(10) \\
{[7]}\end{array}$ \\
\hline Rupure (external) & & & & $\begin{array}{l}1.0 \mathrm{E}-8 / \mathrm{h}-\mathrm{ft}(30) \\
{[7]}\end{array}$ \\
\hline Plugs & & & & $\begin{array}{l}1.0 \mathrm{E}-8 / \mathrm{h}-\mathrm{ft}(30) \\
{[7]}\end{array}$ \\
\hline $\begin{array}{l}\text { Vessel } \\
\text { Tank (Pressurized) } \\
\text { Leakage (external) }\end{array}$ & & $2.3 \mathrm{E}-7 / \mathrm{h}(10)$ & & [3] \\
\hline Rupture (external) & & $7.5 \mathrm{E}-8 \mathrm{~h}(10)$ & & $\begin{array}{l}5.0 \mathrm{E}-9 \mathrm{~h}(30) \\
{[3]}\end{array}$ \\
\hline $\begin{array}{l}\text { Cylinder (Pressurized) } \\
\text { Leakage (external) }\end{array}$ & & & & $\begin{array}{l}1.0 \mathrm{E}-7 / \mathrm{h}(10) \\
{[8]}\end{array}$ \\
\hline Rupture (external) & & & & $\begin{array}{l}5.0 \mathrm{E}-9 / \mathrm{h}(30) \\
{[8]}\end{array}$ \\
\hline $\begin{array}{l}\text { Flange/Gasket } \\
\text { Leakage (extemal) }\end{array}$ & & & & 13] \\
\hline Rupture (extemal) & & & & $\begin{array}{l}1.0 \mathrm{E}-9 / \mathrm{h}(10) \\
{[3]}\end{array}$ \\
\hline
\end{tabular}




\begin{tabular}{|c|c|c|c|c|}
\hline \multirow[t]{2}{*}{ System/Component/Failure Mode } & \multicolumn{4}{|c|}{ Failure Data (Category 1 Sources) } \\
\hline & Category 1 & Category 2 & Category 3 & Recommended $^{\mathrm{b}}$ \\
\hline $\begin{array}{l}\text { Heat Exchanger } \\
\text { Shell/Tube } \\
\text { Fouling (rubes) }\end{array}$ & & & & $\begin{array}{l}1.0 \mathrm{E}-5 / \mathrm{h}(10) \\
{[4]}\end{array}$ \\
\hline Plugs (tubes) & & & & $\begin{array}{l}3.0 \mathrm{E}-6 / \mathrm{h}(10) \\
{[4]}\end{array}$ \\
\hline Leakage (tubes) & & $4.4 \mathrm{E}-5 / \mathrm{h}(1.8)$ & & $\begin{array}{l}1.0 \mathrm{E}-5 / \mathrm{h}(10) \\
{[4]}\end{array}$ \\
\hline$\overline{\text { Rup uire (nibes) }}$ & & $\begin{array}{l}8.5 \mathrm{E}-7 / \mathrm{h}(10) \\
{[1]}\end{array}$ & & $\begin{array}{l}5.0 \mathrm{E}-7 / \mathrm{h}(30) \\
{[4]}\end{array}$ \\
\hline Leakage (shell) & & & & $\begin{array}{l}1.0 \mathrm{E}-6 / \mathrm{h}(10) \\
{[4]}\end{array}$ \\
\hline Rupture (shell) & & & & $\begin{array}{l}5.0 \mathrm{E}-8 / \mathrm{h}(30) \\
{[4]}\end{array}$ \\
\hline $\begin{array}{l}\text { Heater (Electrical) } \\
\text { Fails to heat }\end{array}$ & & $\begin{array}{l}1.0 \mathrm{E}-5 / \mathrm{h}(10) \\
\text { [1] }\end{array}$ & & $\begin{array}{l}1.0 \mathrm{E}-6 / \mathrm{h}(10) \\
{[1]}\end{array}$ \\
\hline Overheass & & & & $\begin{array}{l}3.0 \mathrm{E}-7 / \mathrm{h}(10) \\
{[1]}\end{array}$ \\
\hline Leakage (extermal) & & & & $\begin{array}{l}1.0 \mathrm{E}-6 / \mathrm{h}(10) \\
{[3]}\end{array}$ \\
\hline Kupture (extermal) & & & & $\begin{array}{l}5.0 \mathrm{E}-8 / \mathrm{h}(30) \\
{[3]}\end{array}$ \\
\hline $\begin{array}{l}\text { Vaporizer } \\
\text { Failure }\end{array}$ & & $1.4 E-4 / h(10)$ & & $1.0 \mathrm{E}-4 / \mathrm{h}(10)$ \\
\hline $\begin{array}{c}\text { Air Dyyer } \\
\text { Failure }\end{array}$ & $\begin{array}{l}5.8 \mathrm{E}-6 / \mathrm{h}(10) \\
11]\end{array}$ & $8.9 \mathrm{E}-6 / \mathrm{h}(10)$ & $\begin{array}{l}2.6 \mathrm{E}-7 / \mathrm{h} \\
{[1]}\end{array}$ & $5.0 \mathrm{E}-6 / \mathrm{h}(10)$ \\
\hline $\begin{array}{l}\text { Filter } \\
\text { Plugs }\end{array}$ & & $\begin{array}{l}1.4 \mathrm{E}-5 / \mathrm{h}(1.3) \\
{[2]}\end{array}$ & $\begin{array}{l}3.4 \mathrm{E}-6 / \mathrm{h}(3.4) \\
{[2]}\end{array}$ & $\begin{array}{l}3.0 \mathrm{E}-6 / \mathrm{h}(10) \\
{[1]}\end{array}$ \\
\hline Leakage (internal) & & [2] & [2] & [1] \\
\hline Kupture (intemal) & & [2] & [2] & $\begin{array}{l}5.0 \mathrm{E}-7 / \mathrm{h}(10) \\
{[1]}\end{array}$ \\
\hline $\begin{array}{c}\text { Orifice } \\
\text { Plugs }\end{array}$ & & $\begin{array}{l}2.1 \mathrm{E}-6 / \mathrm{h}(10) \\
{[1]}\end{array}$ & & $\begin{array}{l}1.0 \mathrm{E}-6 / \mathrm{h}(10) \\
{[1]}\end{array}$ \\
\hline
\end{tabular}

Table 6c. Summary of results for compressed gas system. 
a. Aggregated failure rates are mean and error factor (in parentheses). The error factor is the 95 th percentile/50th percentile.

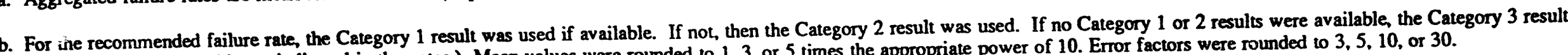

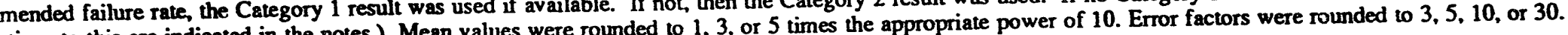

Notes

Category 1

1. Failure rate based on zero failures and may be conservatively high.

\section{Category 2}

1. Failure rate based on zero failures and may be conservatively high.

2. Failure motes not indicated.

\section{Caterory 3}

1. Failure rate based on zero failures and may be conservatively high.

2. Failure modes not indicated.

Recommended

1. Based on comparisons of (limited) compressed gas system data for component groups with the much more extensive water system data (Table 6a), the water system results are appropriate.

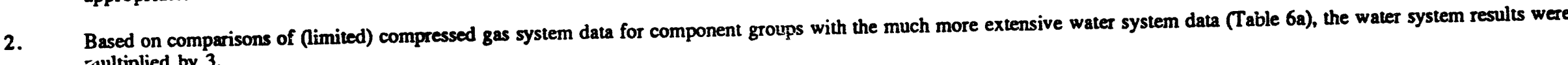
anultiplied by 3 .

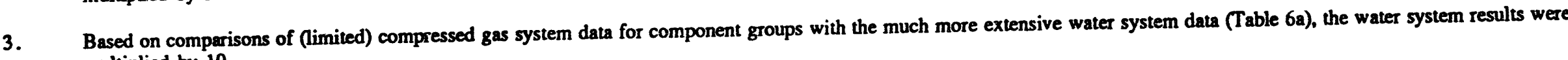
multiplied by 10 .

Based on comparisons of (limited) compressed gas system data for component groups with the much more extensive water system data (Table 6a), the water system results were multiplied by 100.

5. Similar to the water system results (Table 6a), overspeed was assumed to be one-fifth of fails to run.

6. Similar to the water system results (Table 6a), fails to stop was assumed to be comparable to fails to start.

7. Water system piping results were multiplied by 100 .

8. Tank results used. 
Table 6d. Summary of results for HVAC/exhaust system.

\begin{tabular}{|c|c|c|c|c|}
\hline \multirow[t]{2}{*}{ System/Component/Failure Mode } & \multicolumn{4}{|c|}{ Aggregated and Recommended Failure Rates ${ }^{\mathrm{a}}$} \\
\hline & Category 1 & Category 2 & Category 3 & Recommended $\mathbf{b}$ \\
\hline $\begin{array}{l}\text { HVAC/Exhaust } \\
\text { Damper (Standby or Safety) } \\
\text { Manual } \\
\text { Fails to open/close }\end{array}$ & & & & $\begin{array}{l}3.0 \mathrm{E}-3 / \mathrm{d}(10) \\
{[2]}\end{array}$ \\
\hline Plugs & & & & $\begin{array}{l}5.0 \mathrm{E}-7 / \mathrm{h}(10) \\
{[2]}\end{array}$ \\
\hline Leakage (internal) & & & & $\begin{array}{l}1.0 \mathrm{E}-5 / \mathrm{h}(10) \\
{[2]}\end{array}$ \\
\hline Rupture (internal) & & & & $\begin{array}{l}5.0 \mathrm{E}-7 / \mathrm{h}(30) \\
{[2]}\end{array}$ \\
\hline Leakage (extemal) & & & & $\begin{array}{l}1.0 \mathrm{E}-7 / \mathrm{h}(10) \\
{[2]}\end{array}$ \\
\hline Rupture (external) & & & & $\begin{array}{l}5.0 \mathrm{E}-9 / \mathrm{h}(30) \\
{[2]}\end{array}$ \\
\hline $\begin{array}{l}\text { Motor-Operated } \\
\text { Fails to open/close }\end{array}$ & & & & $\begin{array}{l}3.0 \mathrm{E}-2 / \mathrm{d}(10) \\
{[2]}\end{array}$ \\
\hline Spurious operation & & & & $\begin{array}{l}3.0 \mathrm{E}-6 / \mathrm{h}(10) \\
{[2]}\end{array}$ \\
\hline Plugs & & & & $\begin{array}{l}5.0 \mathrm{E}-7 / \mathrm{h}(10) \\
{[2]}\end{array}$ \\
\hline Leakage (internal) & & & & $\begin{array}{l}1.0 \mathrm{E}-5 / \mathrm{h}(10) \\
{[2]}\end{array}$ \\
\hline Rupture (intemal) & & & & $\begin{array}{l}5.0 \mathrm{E}-7 / \mathrm{h}(10) \\
{[2]}\end{array}$ \\
\hline Leakage (extemal) & & & & $\begin{array}{l}1.0 \mathrm{E}-7 / \mathrm{h}(10) \\
{[2]}\end{array}$ \\
\hline Rupiure (external) & & & & $\begin{array}{l}5.0 \mathrm{E}-9 / \mathrm{h}(30) \\
{[2]}\end{array}$ \\
\hline $\begin{array}{l}\text { Air-Operated } \\
\text { Fails to open/close }\end{array}$ & $9.9 \mathrm{E}-3 / \mathrm{d}(6.1)$ & & $\begin{array}{l}1.2 \mathrm{E}-6 / \mathrm{h} \\
{[1]}\end{array}$ & $\begin{array}{l}1.0 \mathrm{E}-2 / \mathrm{d}(30) \\
{[2]}\end{array}$ \\
\hline Spurious operation & $8.2 \mathrm{E}-6 / \mathrm{h}(4.2)$ & & [1] & $\begin{array}{l}1.0 \mathrm{E}-5 / \mathrm{h}(10) \\
{[2]}\end{array}$ \\
\hline Plugs & & & & $\begin{array}{l}5.0 \mathrm{E}-7 / \mathrm{h}(10) \\
{[2]}\end{array}$ \\
\hline Leakage (internal) & & & & $\begin{array}{l}1.0 \mathrm{E}-5 / \mathrm{h}(10) \\
{[2]}\end{array}$ \\
\hline Rupture (intemal) & & & & $\begin{array}{l}5.0 \mathrm{E}-7 / \mathrm{h}(10) \\
{[2]}\end{array}$ \\
\hline
\end{tabular}




\begin{tabular}{|c|c|c|c|c|}
\hline \multirow[t]{2}{*}{ System/Component/Failure Mode } & \multicolumn{4}{|c|}{ Aggregated and Recommended Failure Rates ${ }^{\mathrm{a}}$} \\
\hline & Category 1 & Category 2 & Category 3 & Recommended ${ }^{b}$ \\
\hline Leakage (external) & & & & $\begin{array}{l}1.0 \mathrm{E}-7 / \mathrm{h}(10) \\
{[2]}\end{array}$ \\
\hline Rupture (external) & & & & $\begin{array}{l}5.0 \mathrm{E}-9 / \mathrm{h}(30) \\
{[2]}\end{array}$ \\
\hline $\begin{array}{c}\text { Damper (Control) } \\
\text { Motor-Operated } \\
\text { Fails open }\end{array}$ & & & & $\begin{array}{l}3.0 \mathrm{E}-6 / \mathrm{h}(10) \\
{[1]}\end{array}$ \\
\hline Fails clcsed & & & & $\begin{array}{l}3.0 \mathrm{E}-6 / \mathrm{h}(10) \\
\text { [1] }\end{array}$ \\
\hline Fails to respond & & & & $\begin{array}{l}3.0 \mathrm{E}-6 / \mathrm{h}(10) \\
{[1]}\end{array}$ \\
\hline Plugs & & & & $\begin{array}{l}5.0 \mathrm{E}-7 / \mathrm{h}(10) \\
{[2]}\end{array}$ \\
\hline Leakage (external) & & & & $\begin{array}{l}1.0 \mathrm{E}-7 / \mathrm{h}(10) \\
{[2]}\end{array}$ \\
\hline Rupture (extemal) & & & & $\begin{array}{l}5.0 \mathrm{E}-9 \mathrm{~h}(30) \\
{[2]}\end{array}$ \\
\hline $\begin{array}{l}\text { Air-Operated } \\
\text { Fails open }\end{array}$ & & $\begin{array}{l}8.0 \mathrm{E}-6 / \mathrm{h}(10) \\
{[2]}\end{array}$ & & $\begin{array}{l}3.0 \mathrm{E}-6 / \mathrm{h}(10) \\
\text { [1] }\end{array}$ \\
\hline Fails closed & & [2] & & $\begin{array}{l}3.0 \mathrm{E}-6 / \mathrm{h}(10) \\
\text { [1] }\end{array}$ \\
\hline Fails to respond & & $9.0 \mathrm{E}-7 / \mathrm{h}(10)$ & & $\begin{array}{l}3.0 \mathrm{E}-6 / \mathrm{h}(10) \\
\text { [1] }\end{array}$ \\
\hline Plugs & & & & $\begin{array}{l}5.0 \mathrm{E}-7 / \mathrm{h}(10) \\
{[2]}\end{array}$ \\
\hline Leakage (extemal) & & $4.1 \mathrm{E}-7 / \mathrm{h}(10)$ & & $\begin{array}{l}1.0 \mathrm{E}-7 / \mathrm{h}(10) \\
{[2]}\end{array}$ \\
\hline Rupture (external) & & $2.5 \mathrm{E}-7 / \mathrm{h}(10)$ & & $\begin{array}{l}5.0 \mathrm{E}-9 / \mathrm{h}(30) \\
{[2]}\end{array}$ \\
\hline $\begin{array}{l}\text { Fan/Blower } \\
\text { Motor-Driven } \\
\text { Fails to start }\end{array}$ & $4.4 \mathrm{E}-3 / \mathrm{d}(5.4)$ & $3.3 \mathrm{E}-6 / \mathrm{h}(2.9)$ & $\begin{array}{l}\text { 2.1E-4/d(9.0), } \\
5.0 \mathrm{E}-6 / \mathrm{h}(12)\end{array}$ & $5.0 \mathrm{E}-3 / \mathrm{d}(5)$ \\
\hline Fails to run & $2.9 \mathrm{E}-5 / \mathrm{h}(2 .-1)$ & $5.0 \mathrm{E}-6 / \mathrm{h}(2.8)$ & $5.0 \mathrm{E}-6 / \mathrm{h}(12)$ & $3.0 \mathrm{E}-5 / \mathrm{h}(3)$ \\
\hline Overspeed & & $6.5 \mathrm{E}-7 \mathrm{~h}(1.9)$ & & $\begin{array}{l}5.0 \mathrm{E}-6 / \mathrm{h}(10) \\
{[4]}\end{array}$ \\
\hline Fails to stop & & $3.6 \mathrm{E}-6 / \mathrm{h}(10)$ & & $\begin{array}{l}5.0 \mathrm{E}-3 / \mathrm{d}(10) \\
{[5]}\end{array}$ \\
\hline
\end{tabular}




\begin{tabular}{|c|c|c|c|c|}
\hline \multirow[t]{2}{*}{ System/Component/Failure Mode } & \multicolumn{4}{|c|}{ Aggregated and Recommended Failure Rates ${ }^{\mathrm{a}}$} \\
\hline & Category 1 & Category 2 & Category 3 & Recommended $^{b}$ \\
\hline Leakage (extemal) & & $\begin{array}{l}5.5 \mathrm{E}-8 / \mathrm{h}(10) \\
{[1]}\end{array}$ & & $\begin{array}{l}3.0 \mathrm{E}-7 / \mathrm{h}(10) \\
{[2]}\end{array}$ \\
\hline Rupture (external) & & & & $\begin{array}{l}1.0 \mathrm{E}-8 / \mathrm{h}(30) \\
{[2]}\end{array}$ \\
\hline $\begin{array}{c}\text { Diesel-Driven } \\
\text { Fails to start }\end{array}$ & & & & $\begin{array}{l}1.0 \mathrm{E}-2 / \mathrm{d}(10) \\
{[1]}\end{array}$ \\
\hline Fails to run & & & & $\begin{array}{l}5.0 \mathrm{E}-3 / \mathrm{h}(10) \\
\text { [1] }\end{array}$ \\
\hline Overspeed & & & & $\begin{array}{l}1.0 \mathrm{E}-3 / \mathrm{h}(10) \\
\text { [1] }\end{array}$ \\
\hline Fails to stop & & & & $\begin{array}{l}1.0 \mathrm{E}-2 / \mathrm{d}(10) \\
{[1]}\end{array}$ \\
\hline Leakage (extemal) & & & & $\begin{array}{l}3.0 \mathrm{E}-7 / \mathrm{h}(10) \\
{[2]}\end{array}$ \\
\hline Rupure (extemal) & & & & $\begin{array}{l}1.0 \mathrm{E}-8 / \mathrm{h}(10) \\
{[2]}\end{array}$ \\
\hline $\begin{array}{l}\text { Iructing } \\
\text { Leakage (external) }\end{array}$ & & & & $\begin{array}{l}3.0 \mathrm{E}-7 / \mathrm{h}-\mathrm{ft}(\mathrm{C}) \\
{[3]}\end{array}$ \\
\hline Rupture (extemal) & & & & $1.0 \mathrm{E}-8 / \mathrm{h}-\mathrm{ft}(30)$ \\
\hline Plugs & & & & $\begin{array}{l}1.0 \mathrm{E}-8 / \mathrm{h}-\mathrm{ft}(30) \\
{[3]}\end{array}$ \\
\hline $\begin{array}{l}\text { Heat Exchanget } \\
\text { Air Conditioning Unit/ } \\
\text { Chiller } \\
\text { Fails to start } \\
\end{array}$ & $1.4 \mathrm{E}-2 / \mathrm{d}(10)$ & & $\begin{array}{l}1.0 \mathrm{E}-5 \mathrm{~h}(9.7) \\
{[1]}\end{array}$ & $1.0 \mathrm{E}-2 / \mathrm{d}(10)$ \\
\hline Frils to run & $2.4 \mathrm{E}-5 / \mathrm{h}(10)$ & & [1] & $3.0 \mathrm{E}-5 / \mathrm{h}(10)$ \\
\hline $\begin{array}{l}\text { Fan Cooler Unit } \\
\text { Fails to start }\end{array}$ & $8.4 \mathrm{E}-3 / \mathrm{d}(7.2)$ & & & $1.0 \mathrm{E}-2 / \mathrm{d}(5)$ \\
\hline Fails to run & $8.8 \mathrm{E}-6 / \mathrm{h}(2.9)$ & $3.6 \mathrm{E}-6 / \mathrm{h}(10)$ & & $1.0 \mathrm{E}-5 / \mathrm{h}(3)$ \\
\hline $\begin{array}{l}\text { Heater (Electrical) } \\
\text { Fails to heat }\end{array}$ & & $8.4 \mathrm{E}-7 / \mathrm{h}(2.6)$ & & $1.0 \mathrm{E}-6 / \mathrm{h}(3)$ \\
\hline Overheats & & $1.6 \mathrm{E}-7 / \mathrm{h}(10)$ & & $\begin{array}{l}3.0 \mathrm{E}-7 / \mathrm{h}(10) \\
{[6]}\end{array}$ \\
\hline $\begin{array}{l}\text { Heater (Gas) } \\
\text { Fails to heat }\end{array}$ & & $8.2 \mathrm{E}-4 / \mathrm{h}(10)$ & & $1.0 \mathrm{E}-3 / \mathrm{h}(10)$ \\
\hline
\end{tabular}




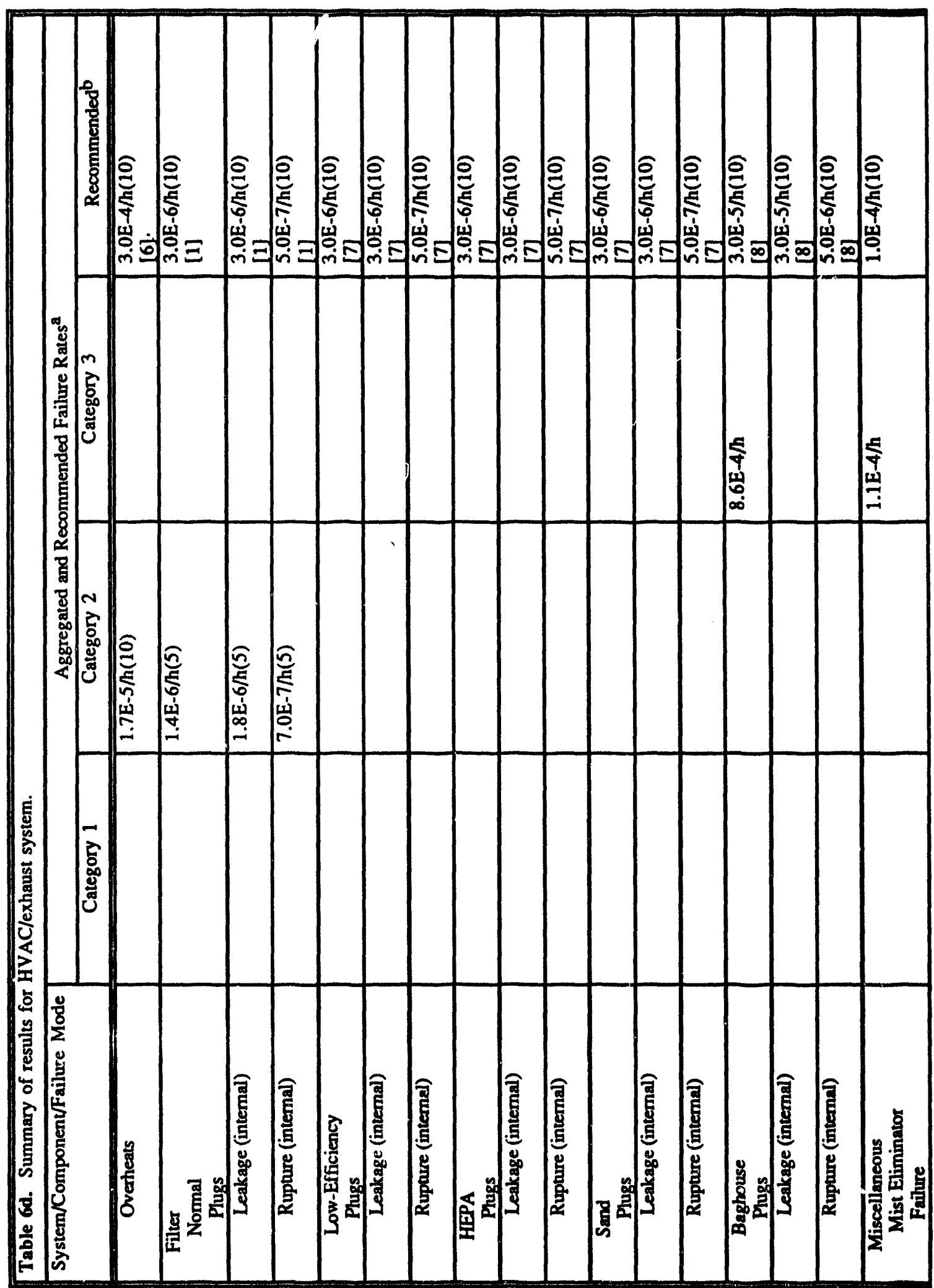

$\stackrel{R}{2}$ 
Table 6d. Summary of results for HVAC/exhaust system.

\begin{tabular}{|c|c|c|c|c|}
\hline \multirow{2}{*}{ System/Component/Failure Mode } & \multicolumn{4}{|c|}{ Aggregated and Recommended Failure Rates $^{\mathrm{a}}$} \\
\cline { 2 - 5 } & Category 1 & Category 2 & \multicolumn{1}{|c|}{ Category 3 $^{\text {Recommended }^{\mathrm{b}}}$} \\
\hline $\begin{array}{c}\text { Scrubber } \\
\text { Failure }\end{array}$ & & $3.2 \mathrm{E}-7 \mathrm{~h}(10)$ & $2.0 \mathrm{E}-4 / \mathrm{h}$ & $\begin{array}{l}1.0 \mathrm{E}-6 / \mathrm{h}(10) \\
{[9]}\end{array}$ \\
\hline $\begin{array}{c}\text { Precipitator } \\
\text { Failure }\end{array}$ & & & $4.6 \mathrm{E}-5 / \mathrm{h}$ & $5.0 \mathrm{E}-5 / \mathrm{h}(10)$ \\
\hline
\end{tabular}

Table 6d. Summary of results for HVAC/exhaust system.

a. Aggregated failure rates are mean and error factor (in parentheses). The error factor is the 95 th percentile/50th percentile.

b. For the recommended failure rate, the Category 1 result was used if available. If not, then the Category 2 result was used. If no Category 1 or 2 results were available, the Category 3 result was used. (Exceptions to this are indicated in the notes.) Mean values were rounded to 1, 3, or 5 times the appropriate power of 10. Error factors were rounded to 3, 5, 10 , or 30.

\section{Notes}

\section{Category 2}

1. Failure rate based on zero failures and may be conservatively high.

\section{Category 3}

1. Failure modes not indicated.

\section{Becommended}

1. Water system results used as appropriate.

2. Based on comparisons of (limiled) HVAC/exhaust system data for component groups with the much more extensive water system data for comparable component groups (Table 6a), the water system results were multiplied by 10.

3. Water system piping results were multiplied by 100 .

4. Similar to the water system results (Table 6a), overspeed was assumed to be one-fifth of fails to run.

5. Similar to the water system results (Table 6a), fails to stop was assumed to be comparable to fails to start.

6. Similar to the water system results (Table 6a), overheats was assumed to be one-fifth as likely as fails to heat. 
7. Normal filter results were used.

8. Based on the Category 3 information, the normal filter results were multiplied by 10.

9. Based on the Category 3 information, the Category 2 result (limited data) was increased. 


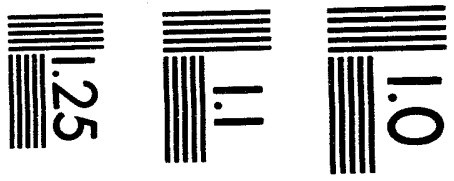

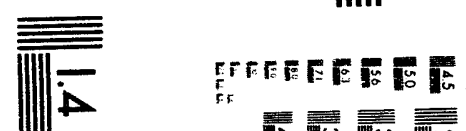

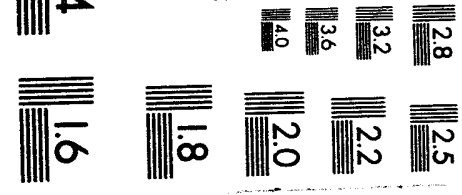




$$
\begin{aligned}
& \omega \\
& \stackrel{o}{\rho} \\
& \omega
\end{aligned}
$$


WSRC-TR-93-262

\begin{tabular}{|c|c|c|c|c|}
\hline \multirow[t]{2}{*}{ System/Component/Failure Mode } & \multicolumn{4}{|c|}{ Aggregated and Recommended Failure Rates ${ }^{a}$} \\
\hline & Category 1 & Category 2 & Category 3 & Recommended $^{b}$ \\
\hline $\begin{array}{l}\text { Electric Power } \\
\text { Generator } \\
\text { Diesel-Driven } \\
\text { Fails to start } \\
\end{array}$ & $1.3 \mathrm{E}-2 / \mathrm{d}(2.2)$ & $\begin{array}{l}3.9 \mathrm{E}-3 / \mathrm{d}(3.0) \\
4.1 \mathrm{E}-5 / \mathrm{h}(3)\end{array}$ & $3.1 \mathrm{E}-2 / \mathrm{d}(11)$ & $1.0 \mathrm{E}-2 / \mathrm{d}(3)$ \\
\hline Fails to nun & $4.1 \mathrm{E}-3 / \mathrm{h}(3.3)$ & $\begin{array}{l}9.7 \mathrm{E}-3 / \mathrm{d}(3.1), \\
1.9 \mathrm{E}-4 / \mathrm{h}(1.2) \\
\end{array}$ & $4.2 \mathrm{E}-3 / \mathrm{h}(24)$ & $5.0 \mathrm{E}-3 / \mathrm{h}(3)$ \\
\hline $\begin{array}{l}\begin{array}{l}\text { Motor-Driven (ac to dc) } \\
\text { Fails to start }\end{array} \\
\end{array}$ & & $1.4 \mathrm{E}-5 / \mathrm{h}(10)$ & $1.8 \mathrm{E}-7 / \mathrm{h}(4.5)$ & $1.0 \mathrm{E}-5 / \mathrm{h}(10)$ \\
\hline Fails to run & $2.1 \mathrm{E}-5 / \mathrm{h}(10)$ & $7.8 \mathrm{E}-6 / \mathrm{h}(10)$ & $3.3 \mathrm{E}-7 / \mathrm{h}(4.3)$ & $3.0 \mathrm{E}-5 / \mathrm{h}(10)$ \\
\hline $\begin{array}{l}\text { Gas-Turbine-Driven } \\
\text { Fails to start } \\
\end{array}$ & $3.8 \mathrm{E}-2 / \mathrm{d}(10)$ & $\begin{array}{l}1.1 \mathrm{E}-2 / \mathrm{d}(3.0), \\
9.9 \mathrm{E}-6 / \mathrm{h}(8.4)\end{array}$ & $9.5 \mathrm{E}-5 / \mathrm{h}(25)$ & $3.0 \mathrm{E}-2 / \mathrm{d}(10)$ \\
\hline Fails to run & $2.6 \mathrm{E}-4 / \mathrm{h}(10)$ & $2.4 \mathrm{E}-5 / \mathrm{h}(8.7)$ & $3.4 \mathrm{E}-4 / \mathrm{h}(25)$ & $3.0 \mathrm{E}-4 / \mathrm{h}(10)$ \\
\hline $\begin{array}{l}\text { Hydro-Turbine-Driven } \\
\text { Fails to start } \\
\end{array}$ & $3.1 \mathrm{E}-3 / \mathrm{d}(10)$ & & & $3.0 \mathrm{E}-3 / \mathrm{d}(10)$ \\
\hline Fails to $\mathrm{nn}$ & & & & $\begin{array}{l}3.0 \mathrm{E}-4 / \mathrm{h}(10) \\
{[1]}\end{array}$ \\
\hline $\begin{array}{c}\text { Battery } \\
\text { Failure } \\
\end{array}$ & $\begin{array}{l}8.0 \mathrm{E}-3 / \mathrm{d}(1.2), \\
8.4 \mathrm{E}-6 / \mathrm{h}(3.7)\end{array}$ & $6.9 \mathrm{E}-7 \mathrm{~h}(10)$ & $4.7 \mathrm{E}-6 / \mathrm{h}(7.0)$ & $1.0 \mathrm{E}-5 / \mathrm{h}(3)$ \\
\hline $\begin{array}{c}\text { Charger } \\
\text { Rectifier } \\
\text { Failure } \\
\end{array}$ & $1.8 \mathrm{E}-5 / \mathrm{h}(1.7)$ & $4.6 \mathrm{E}-6 / \mathrm{h}(2.5)$ & $4.2 \mathrm{E}-6 / \mathrm{h}(3.8)$ & $1.0 \mathrm{E}-5 / \mathrm{h}(3)$ \\
\hline $\begin{array}{l}\text { Bus } \\
\text { Metal-Enclosed } \\
\text { Failure } \\
\end{array}$ & $1.1 \mathrm{E}-7 / \mathrm{h}(10)$ & & $1.3 \mathrm{E}-7 / \mathrm{h}(5)$ & $1.0 \mathrm{E}-7 / \mathrm{h}(5)$ \\
\hline $\begin{array}{l}\text { Bare } \\
\text { Failure }\end{array}$ & & & $8.7 \mathrm{E}-7 / \mathrm{h}(12)$ & $1.0 \mathrm{E}-6 / \mathrm{h}(10)$ \\
\hline $\begin{array}{l}\text { Cable/Joint// } \\
\text { Termination/Jumper } \\
\text { Cable (Copper, 1000ft) } \\
\text { Failure } \\
\end{array}$ & & & $\begin{array}{l}4.5 \mathrm{E}-6 / \mathrm{d}(7.4) \\
3.8 \mathrm{E}-6 / \mathrm{h}(3)\end{array}$ & $3.0 \mathrm{E}-6 / \mathrm{h}(3)$ \\
\hline $\begin{array}{l}\text { Joint (Copper) } \\
\text { Failure }\end{array}$ & & & $3.2 \mathrm{E}-6 \mathrm{~h}(30)$ & $3.0 \mathrm{E}-6 / \mathrm{h}(30)$ \\
\hline $\begin{array}{l}\text { Termination (Copper) } \\
\text { Failure }\end{array}$ & & & $2.6 \mathrm{E}-7 / \mathrm{h}(18)$ & $3.0 \mathrm{E}-7 / \mathrm{h}(10)$ \\
\hline $\begin{array}{l}\text { Jumper (Power) } \\
\text { Failure }\end{array}$ & & & & $\begin{array}{l}5.0 \mathrm{E}-6 / \mathrm{h}(10) \\
{[2]}\end{array}$ \\
\hline
\end{tabular}


WSRC-TR-93-262

Table 6e. Summary of results for electrical distribution system.

\begin{tabular}{|c|c|c|c|c|}
\hline \multirow[t]{2}{*}{ System/Component/Failure Mode } & \multicolumn{4}{|c|}{ Aggregated and Recommended Failure Rates ${ }^{a}$} \\
\hline & Category 1 & Category 2 & Category 3 & Recommended ${ }^{b}$ \\
\hline $\begin{array}{l}\text { Circuit Breaker } \\
\text { General } \\
\text { Fails to open/close }\end{array}$ & $5.6 \mathrm{E}-4 / \mathrm{d}(4.5)$ & $\begin{array}{l}4.0 \mathrm{E}-4 / \mathrm{d}(1.4) \\
4.8 \mathrm{E}-7 / \mathrm{h}(4.8)\end{array}$ & $1.3 \mathrm{E}-3 / \mathrm{d}(13)$ & $5.0 \mathrm{E}-4 / \mathrm{d}(5)$ \\
\hline Spurious operation & $3.8 \mathrm{E}-7 / \mathrm{h}(10)$ & $1.9 \mathrm{E}-7 / \mathrm{h}(4.8)$ & $2.8 \mathrm{E}-6 / \mathrm{h}(20)$ & $3.0 \mathrm{E}-7 / \mathrm{h}(10)$ \\
\hline $\begin{array}{l}\text { Reactor Trip } \\
\text { Fails to open }\end{array}$ & & & & $\begin{array}{l}5.0 \mathrm{E}-3 / \mathrm{d}(5) \\
{[3]}\end{array}$ \\
\hline Spurious operation & & & & $\begin{array}{l}3.0 \mathrm{E}-6 / \mathrm{h}(10) \\
{[3]}\end{array}$ \\
\hline $\begin{array}{l}\text { Relay } \\
\text { Protective } \\
\quad \text { Fails to open/close } \\
\end{array}$ & $1.2 \mathrm{E}-3 / \mathrm{d}(10)$ & $4.2 \mathrm{E}-8 \mathrm{f} / \mathrm{h}(10)$ & $\begin{array}{l}7.1 \mathrm{E}-5 / \mathrm{d}(16) \\
1.9 \mathrm{E}-6 / \mathrm{h}(1.1)\end{array}$ & $1.0 \mathrm{E}-3 / \mathrm{d}(10)$ \\
\hline Spurious operation & & $1.7 \mathrm{E}-7 / \mathrm{h}(10)$ & $1.8 \mathrm{E}-7 / \mathrm{h}(13)$ & $1.0 \mathrm{E}-7 / \mathrm{h}(10)$ \\
\hline $\begin{array}{l}\text { Control } \\
\text { Fails to open/close }\end{array}$ & & & $7.2 \mathrm{E}-6 / \mathrm{d}(1.4)$ & $\begin{array}{l}1.0 \mathrm{E}-4 / \mathrm{d}(10) \\
{[4]}\end{array}$ \\
\hline Spurious operation & & & $3.4 \mathrm{E}-7 \mathrm{~h}(30)$ & $3.0 \mathrm{E}-7 / \mathrm{h}(30)$ \\
\hline $\begin{array}{l}\text { Bistable } \\
\text { Fails to open/close }\end{array}$ & & & & $\begin{array}{l}1.0 \mathrm{E}-5 / \mathrm{d}(10) \\
{[3]}\end{array}$ \\
\hline Spurious operation & & & & $\begin{array}{l}3.0 \mathrm{E}-7 / \mathrm{h}(10) \\
{[3]}\end{array}$ \\
\hline $\begin{array}{l}\text { Switch } \\
\text { Push-Button (Manual) } \\
\text { Fails to open/close }\end{array}$ & & $1.9 \mathrm{E}-\delta / \mathrm{h}(10)$ & $\begin{array}{l}1.3 \mathrm{E}-3 / \mathrm{d}(3) \\
1.8 \mathrm{E}-6 / \mathrm{h}(15)\end{array}$ & $1.0 \mathrm{E}-6 / \mathrm{h}(10)$ \\
\hline Spurious operation & & $1.8 \mathrm{E}-6 / \mathrm{h}(10)$ & $2.6 \mathrm{E}-7 / \mathrm{h}(12)$ & $1.0 \mathrm{E}-6 / \mathrm{h}(10)$ \\
\hline $\begin{array}{l}\text { Rotary (Manual) } \\
\text { Fails to open/close }\end{array}$ & & $6.0 \mathrm{E}-8 / \mathrm{h}(10)$ & $3.1 \mathrm{E}-7 / \mathrm{h}(2)$ & $5.0 \mathrm{E}-8 / \mathrm{h}(10)$ \\
\hline Spurious operation & & $5.0 \mathrm{E}-7 / \mathrm{h}(10)$ & $1.9 \mathrm{E}-7 / \mathrm{h}(2)$ & $5.0 \mathrm{E}-7 / \mathrm{h}(10)$ \\
\hline $\begin{array}{l}\text { Key-Operated (Manual) } \\
\text { Fails to open/close }\end{array}$ & & $3.9 \mathrm{E}-7 / \mathrm{h}(10)$ & $\begin{array}{l}2.3 \mathrm{E}-6 / \mathrm{h} \\
{[1]}\end{array}$ & $3.0 \mathrm{E}-7 / \mathrm{h}(10)$ \\
\hline Spurious operation & & $1.8 \mathrm{E}-6 / \mathrm{h}(10)$ & [1] & $1.0 \mathrm{E}-6 / \mathrm{h}(10)$ \\
\hline $\begin{array}{l}\text { Automatic-Transfer } \\
\text { Fails to open/close }\end{array}$ & $2.0 \mathrm{E}-2 / \mathrm{d}(10)$ & & & $\begin{array}{l}1.0 \mathrm{E}-6 / \mathrm{h}(10) \\
{[5]}\end{array}$ \\
\hline Spurious operation & & & & $1.0 \mathrm{E}-6 / \mathrm{h}(10)$ \\
\hline $\begin{array}{l}\text { Limit } \\
\text { Fails to open/close }\end{array}$ & $\begin{array}{l}\text { 4.0E-5/d(10), } \\
9.2 \mathrm{E}-7 / \mathrm{h}(10)\end{array}$ & & $\begin{array}{l}3.8 \bar{E}-4 / \mathrm{d}(3), \\
6.0 \mathrm{E}-6 / \mathrm{h}(1.6)\end{array}$ & $1.0 \mathrm{E}-6 / \mathrm{h}(10)$ \\
\hline
\end{tabular}


WSRC-TR-93-262

\begin{tabular}{|c|c|c|c|c|}
\hline \multirow[t]{2}{*}{ System/Component/Failure Mode } & \multicolumn{4}{|c|}{ Aggregated and Recommended Failure Rates ${ }^{\mathrm{a}}$} \\
\hline & Category 1 & Category 2 & Category 3 & Recommended $\mathrm{b}$ \\
\hline Spurious operation & & & $1.7 \mathrm{E}-6 / \mathrm{h}(5.1)$ & $1.0 \mathrm{E}-6 / \mathrm{h}(5)$ \\
\hline $\begin{array}{l}\text { Fuse } \\
\text { Fail to open }\end{array}$ & & $1.2 \mathrm{E}-7 / \mathrm{h}(10)$ & $\begin{array}{l}1.3 \mathrm{E}-5 / \mathrm{d}(3) \\
3.5 \mathrm{E}-6 / \mathrm{h}(10) \\
\end{array}$ & $1.0 \mathrm{E}-7 / \mathrm{h}(10)$ \\
\hline Premature opening & & $1.6 \mathrm{E}-8 / \mathrm{h}(10)$ & $1.1 \mathrm{E}-6 / \mathrm{h}(6.1)$ & $1.0 \mathrm{E}-8 / \mathrm{h}(10)$ \\
\hline $\begin{array}{l}\text { Inverter } \\
\text { Failure } \\
\end{array}$ & $\begin{array}{l}7.9 \mathrm{E}-3 / \mathrm{d}(10) \\
1.2 \mathrm{E}-5 / \mathrm{h}(1.9)\end{array}$ & $1.8 \mathrm{E}-5 / \mathrm{h}(6.9)$ & $2.6 \mathrm{E}-5 / \mathrm{h}(12)$ & $1.0 \mathrm{E}-5 / \mathrm{h}(3)$ \\
\hline $\begin{array}{l}\text { Motor } \\
\text { AC } \\
\text { Fails to star }\end{array}$ & & $1.5 \mathrm{E}-5 / \mathrm{h}(3.4)$ & $\begin{array}{l}3.8 \mathrm{E}-4 / \mathrm{d}(3) \\
1.8 \mathrm{E}-5 / \mathrm{h}(17)\end{array}$ & $\begin{array}{l}3.0 \mathrm{E}-4 / \mathrm{d}(3) \\
{[6]}\end{array}$ \\
\hline Fails to run & & $6.0 \mathrm{E}-6 / \mathrm{h}(3.3)$ & $2.4 \mathrm{E}-5 / \mathrm{h}(1.7)$ & $5.0 \mathrm{E}-6 / \mathrm{h}(3)$ \\
\hline Fails to start & & $2.1 \mathrm{E}-5 / \mathrm{h}(3.0)$ & $\begin{array}{l}3.8 \mathrm{E}-4 / \mathrm{d}(3), \\
1.8 \mathrm{E}-5 / \mathrm{h}(8.4) \\
\end{array}$ & $\begin{array}{l}3.0 \mathrm{E}-4 / \mathrm{d}(3) \\
{[6]}\end{array}$ \\
\hline Fails to run & & $8.5 \mathrm{E}-6 / \mathrm{h}(2.8)$ & $2.4 \mathrm{E}-5 / \mathrm{h}(8.4)$ & $1.0 \mathrm{E}-5 / \mathrm{h}(3)$ \\
\hline $\begin{array}{l}\text { Synchro } \\
\text { Failure } \\
\end{array}$ & & $1.4 \mathrm{E}-5 / \mathrm{h}(10)$ & & $1.0 \mathrm{E}-5 / \mathrm{h}(10)$ \\
\hline $\begin{array}{l}\text { Transformer } \\
\text { Power } \\
\text { Failure } \\
\end{array}$ & $8.2 \mathrm{E}-7 \mathrm{~h}(10)$ & 3.5E-7/h(10) & $1.4 \mathrm{E}-6 / \mathrm{h}(5.6)$ & $1.0 \mathrm{E}-6 / \mathrm{h}(10)$ \\
\hline $\begin{array}{l}\text { Instrumentation/Control } \\
\text { Failure }\end{array}$ & $8.9 \mathrm{E}-7 / \mathrm{h}(10)$ & & $3.8 \mathrm{E}-7 / \mathrm{h}(2.8)$ & $1.0 \mathrm{E}-6 / \mathrm{h}(10)$ \\
\hline
\end{tabular}

Table 6e. Summary of results for electrical distribution system.

2. Aggregated failure rates are mean and error factor (in parentheses). The error factor is the 95 th percentile/50th percentile.

b. For the recommended failure rate, the Category 1 result was used if available. If not, then the Category 2 result was used. If no Category 1 or 2 results were available, the Category 3 result was used. (Exceptions to this are indicated in the notes.) Mean values were rounded to 1, 3, or 5 times the appropriate power of 10. Error factors were rounded to 3, 5,10 , or 30.

Notes

\section{Category 3}

1. Faiiure modes nôt induicated. 


\section{Recommended}

1. Gas turbine results used.

2. Jumper modeled as 2 electrical joints. Therefore, the joint result was multiplied by 2 .

3. Estimate taken from Reference 35, Table 4.

4. Based on Category 3 estimate, multiply the protective relay result by 10 .

5. Category 1 data include only 1 failure and very few demands. The highest result from the other switches was used.

6. Per demand result is desired. The Category 3 per hour estimate is similar to the Category 2 estimate (from data), so the Category 3 per demand estimate was assumed to be appropriate. 


\begin{tabular}{|c|c|c|c|c|}
\hline \multirow[t]{2}{*}{ System/Componen//Failure Mode } & \multicolumn{4}{|c|}{ Aggregated and Recommended Failure Rates ${ }^{a}$} \\
\hline & Category 1 & Category 2 & Category 3 & Recommended ${ }^{t}$ \\
\hline $\begin{array}{l}\text { Instrumentation and Control } \\
\text { Alarm/Annunciator } \\
\text { Fails to alarm } \\
\end{array}$ & & $3.6 \mathrm{E}-5 / \mathrm{h}(10)$ & $1.5 \mathrm{E}-6 / \mathrm{h}(15)$ & $3.0 \mathrm{E}-5 / \mathrm{h}(10)$ \\
\hline Spurious operation & & $4.7 \mathrm{E}-6 / \mathrm{h}(10)$ & $8.7 \mathrm{E}-7 / \mathrm{h}(15)$ & $5.0 \mathrm{E}-6 / \mathrm{h}(10)$ \\
\hline $\begin{array}{l}\text { Sensor/Transmitter/ } \\
\text { Transducer/Process Switch } \\
\text { Temperanure } \\
\text { Failure }\end{array}$ & $\begin{array}{l}2.8 \mathrm{E}-4 / \mathrm{d}(3.5) \\
1.1 \mathrm{E}-6 / \mathrm{h}(3.3)\end{array}$ & $8.6 \mathrm{E}-7 / \mathrm{h}(7.9)$ & $8.1 \mathrm{E}-6 / \mathrm{h}(14)$ & $1.0 \mathrm{E}-6 / \mathrm{h}(3)$ \\
\hline $\begin{array}{l}\text { Pressure } \\
\text { Failure } \\
\end{array}$ & $\begin{array}{l}2.8 \mathrm{E}-4 / \mathrm{d}(3.5) \\
1.1 \mathrm{E}-6 / \mathrm{h}(3.3)\end{array}$ & $8.3 \mathrm{E}-7 / \mathrm{h}(3.1)$ & $6.8 \mathrm{E}-6 / \mathrm{h}(17)$ & $1.0 \mathrm{E}-6 / \mathrm{h}(3)$ \\
\hline $\begin{array}{l}\text { Differential Pressure } \\
\text { Failure }\end{array}$ & & $2.7 \mathrm{E}-6 / \mathrm{h}(10)$ & $1.2 \mathrm{E}-4 / \mathrm{h}(16)$ & $3.0 \mathrm{E}-6 / \mathrm{h}(10)$ \\
\hline $\begin{array}{l}\text { Fow } \\
\text { Failure }\end{array}$ & & $2.9 \mathrm{E}-6 / \mathrm{h}(2.0)$ & $3.2 \mathrm{E}-5 / \mathrm{h}(15)$ & $3.0 \mathrm{E}-6 / \mathrm{h}(3)$ \\
\hline $\begin{array}{l}\text { Level } \\
\text { Failure }\end{array}$ & & $5.3 \mathrm{E}-7 / \mathrm{h}(3.7)$ & $6.4 \mathrm{E}-6 / \mathrm{h}(7.7)$ & $5.0 \mathrm{E}-7 / \mathrm{h}(3)$ \\
\hline $\begin{array}{c}\text { Humidity } \\
\text { Failure }\end{array}$ & & $1.2 \mathrm{E}-5 / \mathrm{h}(10)$ & $4.2 \mathrm{E}-6 / \mathrm{h}(3.9)$ & $1.0 \mathrm{E}-5 / \mathrm{h}(10)$ \\
\hline $\begin{array}{l}\mathrm{pH} \\
\text { Failure } \\
\end{array}$ & & & $5.8 \mathrm{E}-7 / \mathrm{h}(5.0)$ & $5.0 \mathrm{E}-7 / \mathrm{h}(5)$ \\
\hline $\begin{array}{l}\text { Oxygen Concentration } \\
\text { Failure }\end{array}$ & & $9.5 \mathrm{E}-6 / \mathrm{h}(10)$ & & $1.0 \mathrm{E}-5 / \mathrm{h}(10)$ \\
\hline $\begin{array}{l}\mathrm{CO}_{2} \text { Concentration } \\
\text { Failure }\end{array}$ & & & $9.8 \mathrm{E}-5 / \mathrm{h}(10)$ & $1.0 \mathrm{E}-4 / \mathrm{h}(10)$ \\
\hline $\begin{array}{l}\text { Hydrogen Concentration } \\
\text { Failure }\end{array}$ & & & & $1.0 \mathrm{E}-5 / \mathrm{h}(3)$ \\
\hline $\begin{array}{l}\text { Nitrogen Concentration } \\
\text { Failure }\end{array}$ & & & & $\begin{array}{l}1.0 \mathrm{E}-5 / \mathrm{h}(3) \\
{[1]}\end{array}$ \\
\hline $\begin{array}{l}\text { Hydrocarbon Concentration } \\
\text { Failure }\end{array}$ & & $7.9 \mathrm{E}-6 / \mathrm{h}(2.2)$ & & $1.0 \mathrm{E}-5 / \mathrm{h}(3)$ \\
\hline $\begin{array}{l}\text { Helium Concentration } \\
\text { Failure }\end{array}$ & & & & $\begin{array}{l}1.0 \mathrm{E}-5 \mathrm{~h}(3) \\
\text { [1] }\end{array}$ \\
\hline $\begin{array}{l}\text { Speed } \\
\text { Failure }\end{array}$ & & & $1.7 \mathrm{E}-6 / \mathrm{h}(8.1)$ & $1.0 \mathrm{E}-6 / \mathrm{h}(10)$ \\
\hline $\begin{array}{c}\text { Seismic } \\
\text { Failure }\end{array}$ & & & $1.6 \mathrm{E}-6 / \mathrm{h}(4)$ & $1.0 \mathrm{E}-6 / \mathrm{h}(5)$ \\
\hline $\begin{array}{c}\text { Radiation } \\
\text { Failure }\end{array}$ & & & $6.3 \mathrm{E}-6 / \mathrm{h}(4.7)$ & $5.0 \mathrm{E}-6 / \mathrm{h}(5)$ \\
\hline
\end{tabular}


WSRC-TR-93-262

\begin{tabular}{|c|c|c|c|c|}
\hline \multirow[t]{2}{*}{ System/Conlponent/Failure Mode } & \multicolumn{4}{|c|}{ Aggregated and Recommended Failure Rates ${ }^{a}$} \\
\hline & Category 1 & Category 2 & Category 3 & Recommended ${ }^{b}$ \\
\hline $\begin{array}{l}\text { Indicator } \\
\text { Failure } \\
\end{array}$ & & $1.5 \mathrm{E}-5 / \mathrm{h}(10)$ & $1.1 \mathrm{E}-4 / \mathrm{h}(22)$ & $1.0 \mathrm{E}-5 / \mathrm{h}(10)$ \\
\hline $\begin{array}{l}\text { Amplifier } \\
\text { Failure }\end{array}$ & & & & $\begin{array}{l}5.0 \mathrm{E}-6 / \mathrm{h}(10) \\
{[2]}\end{array}$ \\
\hline $\begin{array}{l}\text { Modifier/Signal Conditioner } \\
\text { Failure }\end{array}$ & & & $3.3 \mathrm{E}-7 / \mathrm{h}(2.1)$ & $3.0 \mathrm{E}-7 / \mathrm{h}(3)$ \\
\hline $\begin{array}{l}\text { Logic Module } \\
\text { Failure } \\
\end{array}$ & & & $3.7 \mathrm{E}-6 / \mathrm{h}(7.7)$ & $3.0 \mathrm{E}-6 / \mathrm{h}(5)$ \\
\hline $\begin{array}{c}\text { Recorder } \\
\text { Failure }\end{array}$ & & & $3.8 \mathrm{E}-5 / \mathrm{h}(22)$ & $3.0 \mathrm{E}-5 / \mathrm{h}(30)$ \\
\hline $\begin{array}{c}\text { Sampler } \\
\text { Failure } \\
\end{array}$ & & $1.2 \mathrm{E}-5 / \mathrm{h}(10)$ & & $1.0 \mathrm{E}-5 / \mathrm{h}(10)$ \\
\hline $\begin{array}{c}\text { Analyzer } \\
\text { Failure } \\
\end{array}$ & & $6.0 \mathrm{E}-6 / \mathrm{h}(10)$ & $1.3 \mathrm{E}-3 / \mathrm{h}(26)$ & $5.0 \mathrm{E}-6 / \mathrm{h}(10)$ \\
\hline $\begin{array}{l}\text { Timer } \\
\text { Failure } \\
\end{array}$ & & & & $\begin{array}{l}5.0 \mathrm{E}-6 / \mathrm{h}(10) \\
{[3]}\end{array}$ \\
\hline $\begin{array}{l}\text { Gas Chromatograph } \\
\text { Failure }\end{array}$ & & $7.3 \mathrm{E}-5 / \mathrm{h}(10)$ & & $5.0 \mathrm{E}-5 / \mathrm{h}(10)$ \\
\hline $\begin{array}{l}\text { Voltage Regulator } \\
\text { Failure }\end{array}$ & & $3.2 \mathrm{E}-6 / \mathrm{h}(10)$ & & $3.0 \mathrm{E}-6 / \mathrm{h}(10)$ \\
\hline $\begin{array}{l}\text { Transmitter } \\
\text { Failure }\end{array}$ & $2.2 \mathrm{E}-6 / \mathrm{h}(1.4)$ & & $1.0 \mathrm{E}-5 / \mathrm{h}(30)$ & $3.0 \mathrm{E}-6 / \mathrm{h}(10)$ \\
\hline $\begin{array}{l}\text { Transducer } \\
\text { Failure }\end{array}$ & & $9.2 \mathrm{E}-7 / \mathrm{h}(10)$ & $6.3 \mathrm{E}-5 / \mathrm{h}(15)$ & $1.0 \mathrm{E}-6 / \mathrm{h}(10)$ \\
\hline $\begin{array}{l}\text { Programmable Logic Controller } \\
\text { Failure }\end{array}$ & & $3.2 \mathrm{E}-5 / \mathrm{h}(10)$ & & $3.0 \mathrm{E}-5 / \mathrm{h}(10)$ \\
\hline
\end{tabular}

Table 6f. Summary of results for instrumentation and control system.

a. Aggregated failure rates are mean and error factor (in parentheses). The enror factor is the 95 th percentile/50th percentile.

b. For the recommended failure rate, the Category 1 result was used if available. If not, the Category 2 result was used. If no Category 1 or 2 results were available, the Category 3 result was b. For the rections to this indicated in the notes.) Mean Values were rounded to 1,3 , or 5 times the appropriate power of 10 . Error factors were rounded to 3,5, 10, or 30. 


\section{Notes}

Recommended

1. $\mathrm{O}_{2}$ result used.

2. Result obtained from Reference 35, Table 4.

3. Representative estimate, based on the other results in the table. 
Table 7. Naming scheme component codes.

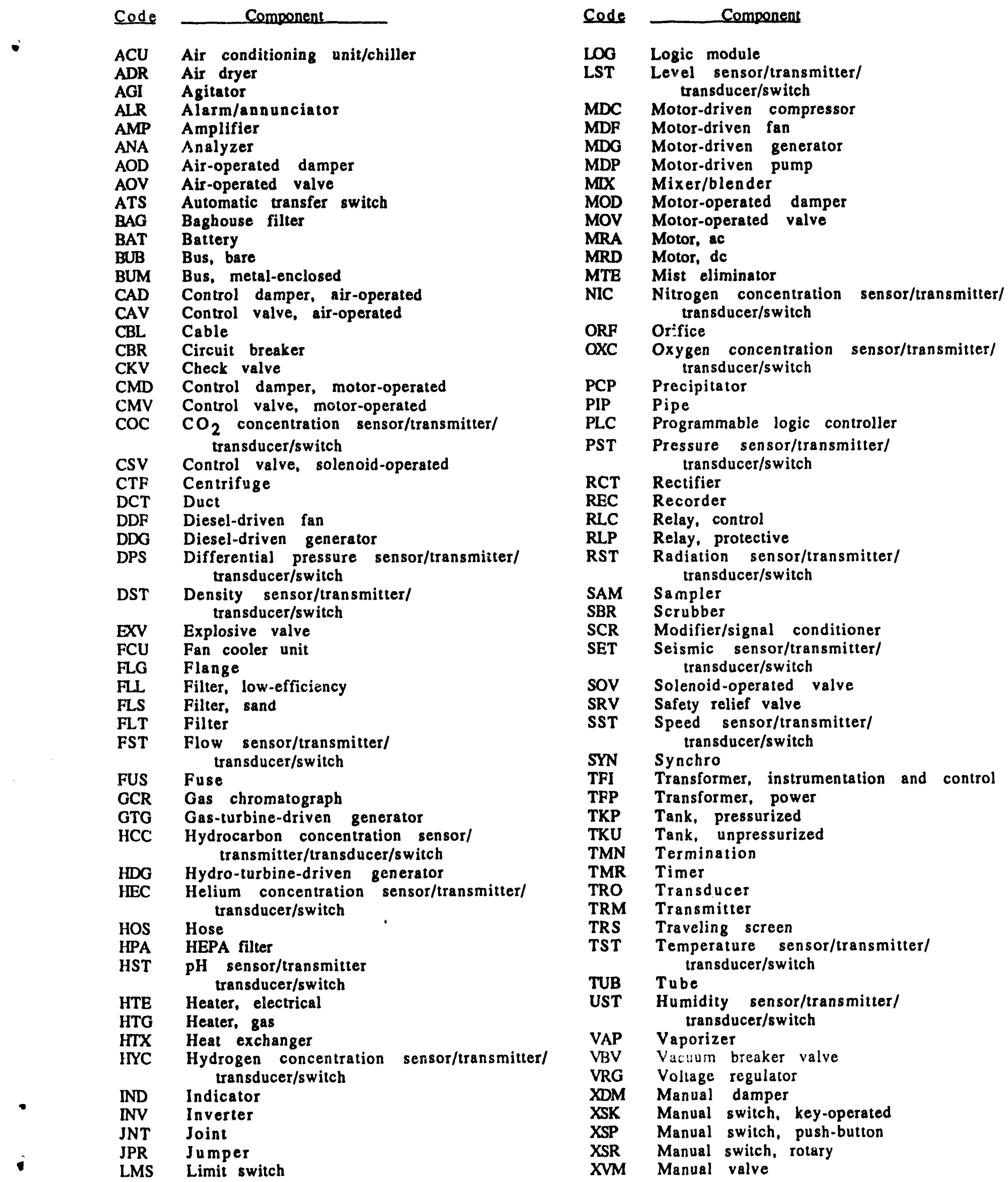


Table 8. Naming scheme failure mode codes.

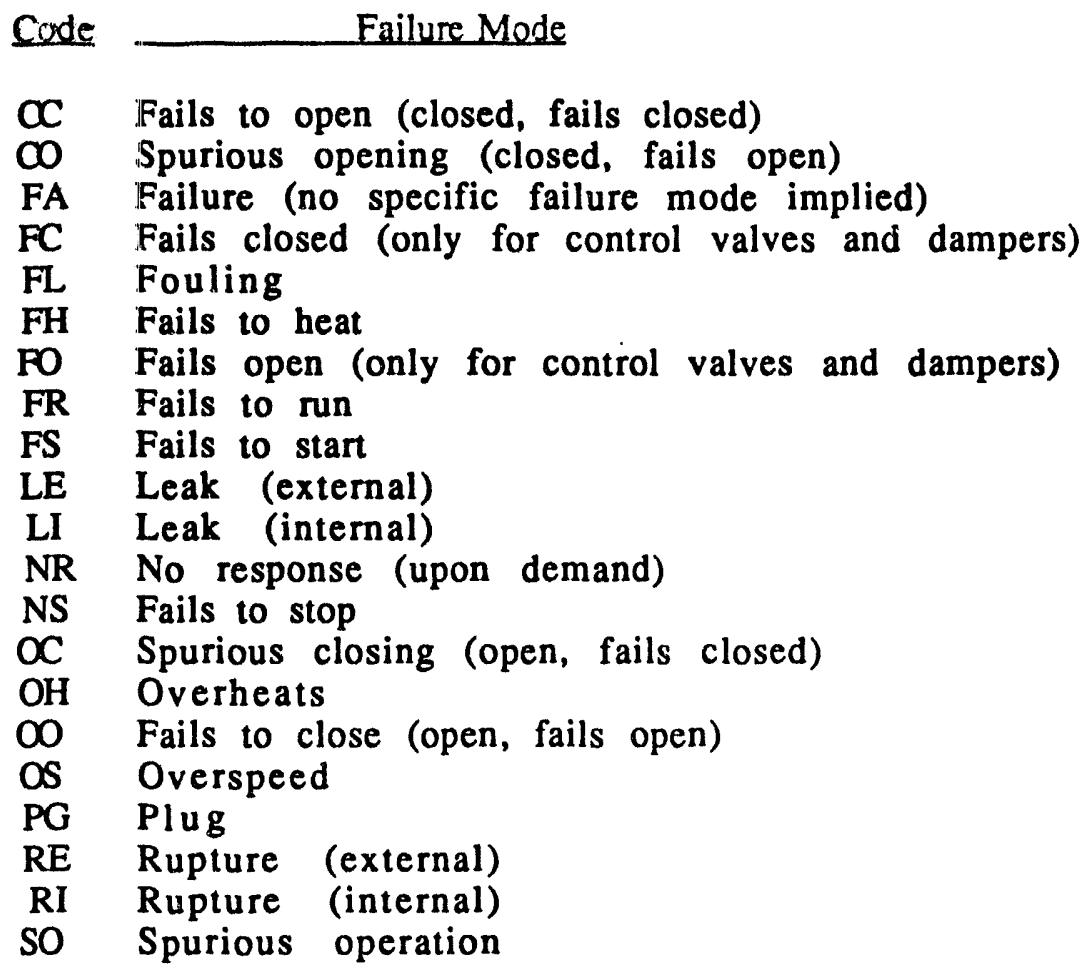

Table 9. Naming scheme system codes.

\begin{tabular}{ll} 
Code & \multicolumn{2}{c}{ Type of System } \\
C & Chemical process \\
E & Electrical distribution \\
G & Compressed gas \\
H & HV $\Lambda$ C/exhaust \\
I & Instrumentation and control \\
W & Water
\end{tabular}



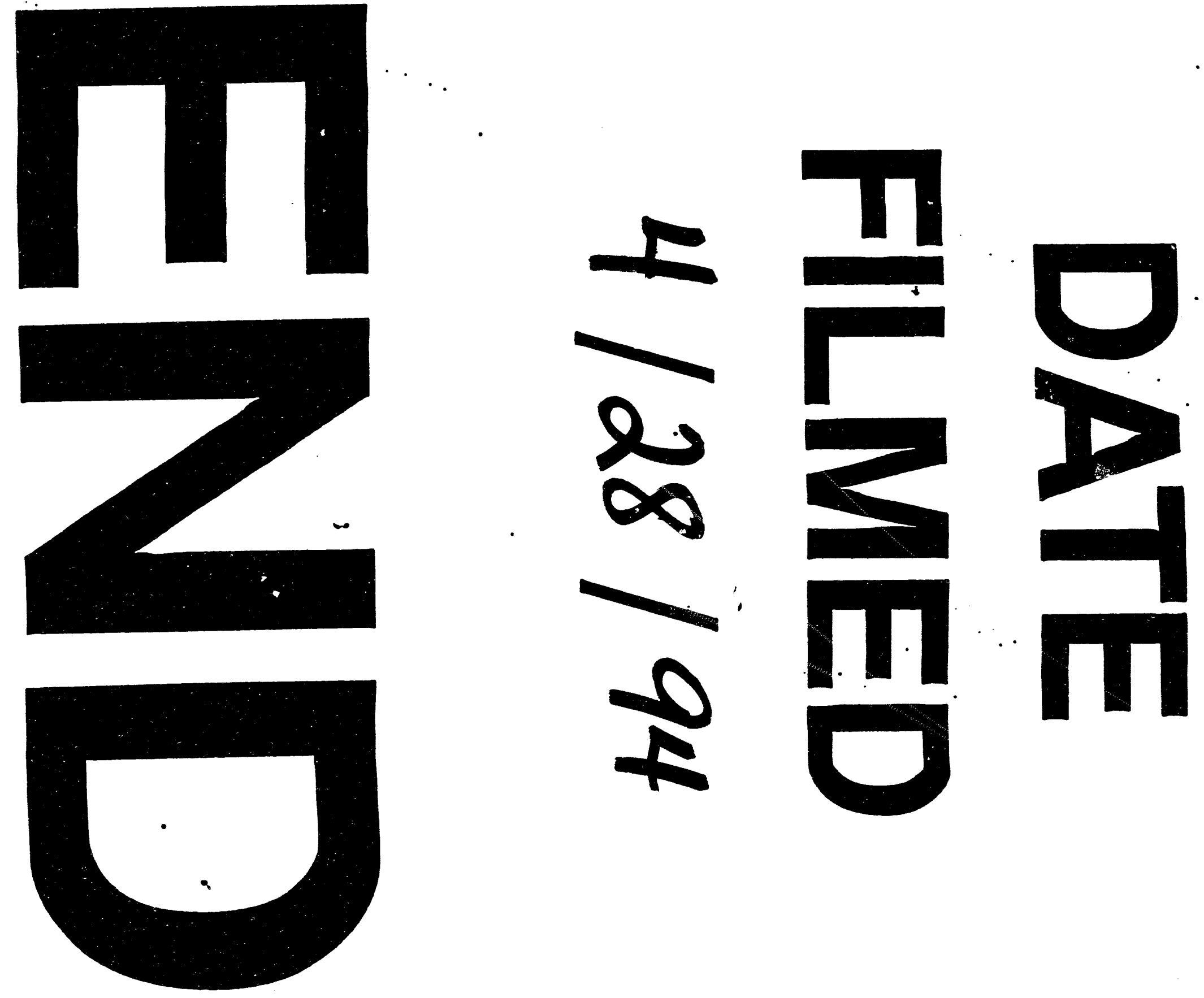


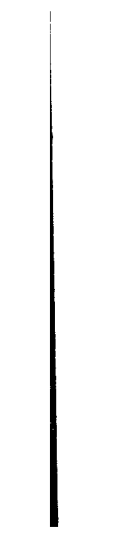

